LBL- 34386

UC- 213

\title{
Current-Potential Characteristics of Electrochemical Systems
}

\author{
Vincent S. Battaglia \\ $\mathrm{Ph}$.D. Thesis
Department of Chemical Engineering
University of California \\ and
Materials Sciences Division
Lawrence Berkeley Laboratory
University of California \\ Berkeley, California 94720
}

July 20, 1993

This work was supported by the Assistant Secretary for Conservation and Hybrid Propulsion Division of the U ortation Technologies, Electric and tract No. DE-AC03-76SF00098. 


\author{
Abstract \\ Current-Potential Characteristics of Electrochemical Systems \\ by \\ Vincent Santo Battaglia \\ Doctor of Philosophy in Chemical Engineering \\ University of California, Berkeley \\ Professor John S. Newman, Chair
}

This dissertation contains investigations in three distinct areas. Chapters 1 and 2 provide an analysis of the effects of electromagnetic phenomena during the initial stages of cell discharge. Chapter 1 includes the solution to Maxwell's equations for the penetration of the axial component of an electric field into an infinitely long cylindrical conductor. This work investigates two time regimes: the "short time" and the "diffusive time". During the short time the current increases at a rate proportional to the time, and during the diffusive time it increases at a rate proportional to the square root of time. Also included in this chapter are mathematical expressions that describe the manner in which the electric field penetrates; a conductive medium during the two time regimes. Chapter 2 contains the analysis of the conductor included in a radial circuit. The time constant for the discharge of the circuit is depicted as a function of the size of the system and the important physical parameters. Also included are analytic approximations of the time constant and a comparison to the lumped parameter approximation of 
inductance.

Chapter 3 provides a complete description of the equations that describe the growth of an oxide film. A finite difference program was written to solve the equations. The system investigated is the iron/iron oxide in a basic, aqueous solution. Figures are presented which provide a comparison of the simulations to the experimental data provided in the literature. Discussion of the quality of the fit is also provided. Ultimately, we found that at low voltages iron dissolves preferentially to form ferrous ions and at higher voltages reacts to form ferrous oxide.

Chapters 4 and 5 include the experimental attempts of replacing formaldehyde with an innocuous reducing agent for electroless deposition. In chapter 4, current-versus-voltage curves are provided for a sodium thiosulfate bath in the presence of a copper disk electrode. Also provided are the cathodic polarization curves of a copper/EDTA bath in the presence of a copper electrode. Explanations for the poor performance of thiosulfate as a reducing agent for copper are included. Chapter 5 contains the experimental results of work done with sodium hypophosphite as a reducing agent. Mixed-potentialversus-time curves for solutions containing various combinations of copper sulfate, nickel cloride, and hypophosphite in the presence of a palladium disk electrode provide an indication of the reducing power of the solutions. It appears that the hypophosphite is preferentially oxidized on nickel and its oxidation is inhibited on copper. 
Table of Contents

Title Page

Approval Page

Abstract

Table of Contents

Preface

vi

Acknowledgements

ix

Chapter 1: Magnetic Field Effects in High-Power Batteries

I: The Penetration of an Electric Field into a Cylindrical Conductor

Foreword

Introduction

The Electromagnetic System

Full Solution

Diffusion Solution

Inner Solution

Current

Summary

List of Symbols

References

Chapter 2: Magnetic Field Effects in High-Power Batteries

II. Time Constant of a Radial Circuit Terminated by a Cylindrical Cell with Inductance

Foreword

Introduction

Analytic Approach 
Transmission-Iine Analysis 29

Case 1: Outer Conductors of Infinite Conductivity 34 Initial Conditions $\quad 35$ Boundary Conditions $\quad 35$ $\begin{array}{lr}\text { Solution } & 38\end{array}$

Time Constants $\quad 38$

Case 2: Outer Conductors of Finite Conductivity 41 Solution $\quad 44$

Time Constants $\quad 44$

$\begin{array}{lr}\text { Discussion } & 48\end{array}$

Conclusions $\quad \cdot 49$

List of Symbols $r$

References $r$

Chapter 3: The Modeling of a Growing Oxide Film 53

Foreword $\quad 53$

Introduction $\quad 53$

General System Description $\quad 57$

Equations $\quad 61$

Mole Flux Balance $\quad 61$

Summing Equations $\quad 64$

Poisson's Equation $\quad 65$

$\begin{array}{ll}\text { Velocity } & 66\end{array}$

$\begin{array}{lr}\text { Boundary Conditions } & 68\end{array}$

Mole Balance $r$

Potential $\quad 74$

$\begin{array}{ll}\text { Velocity } & 75\end{array}$

Initial Conditions $\quad 76$

$\begin{array}{ll}\text { Reactions } & 77\end{array}$

Electric Fields on Homogeneous Reactions 77

Thermodynamic Consistency of Heterogeneous Reactions 79

Solving Computationally $\quad 81$

Coordinate Transformation $\quad 81$

Equations in Finite Difference $\quad 82$

Iron/Iron Oxide System $\quad 83$

Summary of Earlier Work as Provided by Vetter

Model $\quad 85$

$\begin{array}{lr}\text { System Parameters } & 89\end{array}$

Results and Discussion $\quad 94$

Equilibrium $\quad 95$

Comparison with Experimental Data 97

Underpotential Deposition 108

$\begin{array}{lr}\text { Summary } & 113\end{array}$

List of Symbols $r$\begin{tabular}{ll}
115 \\
\hline
\end{tabular}

$\begin{array}{lr}\text { References } & 118\end{array}$

$\begin{array}{lr}\text { Appendix } & 123\end{array}$

$\begin{array}{ll}\text { Program } & 123\end{array}$

Main Program: iren $r 26$

Data File: iren.dat $r$ 
Chapter 4: The Oxidation of Thiosulfate on Copper for
Electroless Deposition

Foreword

Introduction

Experimental

Results

Reduction of Copper Sulfate in the Presence 182

Oxidation of Sodium

Results of Combi

Presence of Copper

Discussion

Conclusions

References

Chapter 5: Electroless Deposition of Copper by
Hypophosphite and Nickel Solutions

Foreword

Introduction

Experimental

Results and Discussion

Nickel Sulfate Discussion

Further Discussions 


\section{Preface}

This dissertation consists of three distinct subjects covered over five chapters. The first investigation is on the effect the magnetic field, or inductance, has on the charging of a system. Chapter one illustrates the manner in which an electric field penetrates an infinite cylindrical conductor that short circuits two perfect plates. That analysis provides part of the solution to finding the time constant of a complete circuit that is instantaneously subject to a constant potential source. In chapter 2 , a cylindrical conductor is included in a radial circuit. Solution to a transmission-line equation provides the time constant of the system as a function of the various physical parameters. The second subject considered is the modeling of a growing oxide film. Chapter 3 provides a physical description of the system and the equations governing the transport in the oxide. This analysis is then applied to the iron/iron oxide system. Results generated from a computer program are compared with experimental evidence reported in the literature. The third area of research is electroless deposition of copper. This work was exploracory. The goal was to find a workable yet less hazardous replacement for the widely used reducing agent formaldehyde. The end result maintains that under the piesent conditions neither sodium thiosulphate nor sodium hypophosphate is the answer. A significant and sustained amount of copper deposition was never achieved. Chapter 4 contains the polarization curves of the copper deposition and thiosulphate oxidation. Chapter 5 contains the 
results of using hypophosphite with various amounts of nickel.

This thesis can also be considered a monograph of the three primary techniques that are available to the engineer for systems analysis: an analytical approach, used in the magnetic field work; a computational approach, applied in the oxide film work; and an experimental approach, undertaken in the electroless deposition work. To perform a complete analysis of the magnetic field effects we solved Maxwell's equations of electromagnetism using a perturbation analysis. This method divided the problem into a short time and long time analysis. To obtain the time constant of the circuit configuration, concepts in Laplace transforms, Duhamel's superposition, and residue theory were applied. Upon developing the equations for describing oxide film growth, a computer program was prepared. The program was written in a general manner such that any number of species, phases, and homogeneous and heterogeneous reactions could be considered. Preparing such a model required a general algorithm for combining mole balance equations such that any reactions that were considered to be at equilibrium were eliminated from all but one mole balance equation. That equation was then replaced by an equilibrium expression. The substitution of Poisson's equation for the electroneutrality equation introduced the complexities associated with rapid variations in concentration and potential distributions near interfaces. These variations, which can severely hinder convergence, were minimized by a number of programming techniques. The exploratory work in electroless deposition has not only allowed an enhance- 
ment of my experimental skills, but has exposed me to the methodology involved in starting any project from ground zero.

This work has, accordingly, provided a vehicle for exploring a wide breath of physical phenomena and has been a valuable learning experience in the methods of problem solving. I am appreciative of the experience.

V. S. Battaglia 


\section{Acknowledgements}

This space is set aside for the author to express thanks to those who made contributions towards his achieving the goal of becoming a Ph.D. This space has no format or guidelines; I am on my own.

As anyone will attest, I am no genius. I made it to this point by a lot of hard work and by being fortunate enough to be surrounded by many good people who had my best interests at heart. It is impossible to thank all of them, but I can thank those who provided the most significant assistance.

Growing up in Baltimore, I did not sit around dreaming of the day when I would become a Chemical Engineering Ph.D. As a matter of fact, I thought very little about my future. The one thing I did do was to listen to my parents say "Stay in school and everything will work itself out." Although they were probably not the first parents to utter these words. they were the people who said them to me. They are the source of my continuing success and I love them.

Some people play major factors in another's life and know little of the outcome. Two people are indirectly, directly responsible for my ending up at Berkeley and should be acknowledged: my junior high school guidance counselor, who got me out of her poor school and into the college preparatory curriculum at the Baltimore Polytechnic Institute; and Professor William Schwarz, who told me to go to the best graduate school that would accept me. Thank you for your guidance. The direct influence on my decision to come to Berkeley rests 
with my father, who, while lying partially asleep in bed, had the wisdom to ask the immortal phrase "Minnesota instead of California?" Thanks dad.

Coming to Berkeley was the first time I lived away from my parents: a very scary experience. If it were not for Joseph D. Puglisi ("Joe D."), I seriously doubt that I would have survived here. He helped me not only to adjust to my new surroundings but through my Master's thesis, my Preliminary Examination, and my Qualifying Examination. Thank you is not strong enough. I thrank you, I thrunk you.

The choice of an advisor is a major decision that influences the remainder of your life. Alan West, having fought his internal instincts to not allow his room mate to also share the same lab, convinced me to work in Prof. Newman's research group. Thank you, Alan, for fighting those instincts and providing excellent advice.

Finally, this Ph.D thesis was five and a half years in the making. In those five and a half years I have experienced significant growth, both intellectually and personally. John Newman deserves most of the credit for this maturation. His patience and honesty have deeply enriched my life.

This work was supported by the Assistant Secretary for Conservation and Renewable Energy, Office of Transportation Technologies, Electric and Hybrid Propulsion Division of the U.S. Department of Energy under Contract No. DE-AC03-76SF00098. 


\author{
Chapter :- \\ Magnetic Field Effects in High-Power Batteries \\ I: The Penetration of an Electric Field \\ into a Cylindrical Conductor
}

\begin{abstract}
Foreword
The penetration of the axial component of an electric field into a cylindrical conductor is described by an asymptotic solution method for long and short times. The development of the respective current distributions allows for a mathematical comparison of the solution schemes and indicates that the current initially increases at a rate proportional to time until a time of order $\epsilon / \sigma$, subsequently at a rate proportional to the square root of time, and finally levels off exponentially to the steady-state value. Criteria for th. proper omission of the displacement current are also given.
\end{abstract}

\title{
Introduction
}

In the 70 years since Kapitza ${ }^{1}$ used an electrochemical system (a lead-acid bipolar battery) for delivering short pulses of high-power, the design of batteries for this purpose has drawn little attention. However, the ever increasing reliance of the business sector on computer and telecommunication systems has enticed a renewed effort in research of rapid-discharge, high, back-up power batteries. E. Willihnganz ${ }^{2}$ studied the effect of high rates of discharge on the negative plate of a lead-acid battery. Gibbard ${ }^{3}$ has designed and tested 
a number of high-power lithium batteries and has presented an overall design criterion. 4 And LaFollette and Bennion discuss the design fundamentals of high-power, pulse-discharged lead acid batteries in terms of experiments ${ }^{5}$ and modeling. ${ }^{6}$ Researchers are now also asking what is the minimum time required to bring a battery to maximum power.

Inductance, used to denote the effect of a rapidly varying electric field which produces a varying magnetic field which counters with an opposing electric field, governs the rise time of the current. This phenomenon falls under the heading of electrodynamics. LaFollette and Bennion ${ }^{5}$ mention that the rigorous way to incorporate magnetic field effects in a battery model is to solve Maxwell's equations of electromagnetism simultaneously with the equations that govern the battery performance. Due to the complicated nature of the equations of electrodynamics and the associated boundary conditions, this is something most modelers would like to avoid. LaFollette et al. did not include the inductance in their model after demonstrating that their system was small enough that the inductance effect occurred within the first microsecond and that they were more interested in the 10 to 1000 us range. Methods of simplifying the equations for handling the inductance while not neglecting it entirely do exist. Mckinney et al. ${ }^{7}$ include a magnetic field effect of the circuit when analyzing the current from a power source by including a lumped inductance term in their circuit-theory model. Cahan et al. 8 performed a parametric study of the impedance of a gen- 
eric power source as a function of the frequency of the signal. The power source was approximated as a modified, semi-infinite, stripline. Inductance was included by means of a skin-effect. The skindepth is a measure of the depth of penetration of a sinusoidal signal and is a function of the frequency and conductivity. (For a sinusoidal signal, 95 percent of the signal is limited to 3 skindepths from the surface.) The skin-depth analysis is applicable for a sinusoidal signal; however, an analogous treatment for a stepped current has not yet been presented. Moreover, these techniques only provide for an adjustment of the total current as a function of time, they do not provide a description of the instantaneous current distribution. The current distribution can play an important role in battery design if a large battery is required for high currents and only a fraction is utili.ed.

In part I of our study, we shall demonstrate the manner in which an electric field penetrates a cylindrical conductor when the conductor is instantaneously subjected to a constant electric field at its surface. This work provides a first approximation to the instantaneous current distribution in a bipolar battery, the typical battery design chosen for delivering high power. In part II, the cylindrical conductor is included in a radial circuit. A transmission-line analysis is used in conjunction with the above treatment to determine the time constant of the complete system. The importance of addressing the complete circuit lies in the fact that the electromagnetic field distribution is not a localized entity but is a function of the 
entire circuit geometry.

\section{The Electromagnetic System}

The transition of an electrical signal from one amplitude and frequency to another begins at the source of the disturbance and propagates to the rest of the electrical configuration. The new form of the signal travels at the speed of light between the electrical conductors used to direct the electrical power from the source to the intended load. During the initial stages of the transition, the charge on the conductors distributes itself such that the potential around the circuit is consistent with Ohm's law. (Further comments on the initial stages of current start-up are provided by Rosser ${ }^{9}$ and Heald. ${ }^{10}$,

For conductors of finite conductivity, the electric field penetrates the conductors to within a few skin depths, for an alternating signal, and throughout the conductors, for a direct signal. The rate at which the new signal can penetrate the conductors determines the rate to a periodic or steady state. Using Maxwell's equations, it is the purpose of this paper to describe the penetration of the axial electric field into an electrically conducting medium.

It is well-known that Maxwell's four coupled equations of electromagnetism reduce to two, noncoupled modified wave equations in a linear, homogeneous, conducting medium: 


$$
\begin{aligned}
& \nabla^{2} E=\mu \epsilon \frac{\partial^{2} E}{\partial t^{2}}+\mu \sigma \frac{\partial E}{\partial t}, \\
& \nabla^{2} B=\mu \epsilon \frac{\partial^{2} B}{\partial t^{2}}+\mu \sigma \frac{\partial B}{\partial t} .
\end{aligned}
$$

The solution to these equations for the transient electric field distribution in the conductors and surrounding medium for a finite geometry with a load and source is difficult to obtain. Since we are interested here in the time it takes for the penetration of the electrical signal into the power source, we shall address the specific problem of a step in an electrical signal propagating down an infinitely long coaxial cable. The radial component of the electrical signal propagates between the two conductors at the speed of light while the axial component propagates radially into the inner and outer conductors. With this configuration, the problem of determining the penetration of the electric field into the transmission line is virtually reduced to the mathematically one-dimensional problem of the penetration of an axial electric field component into an infinitely long cylindrical conductor.

From this analysis we shall assume that the bipolar battery configuration can be approximated by the properties of the central wire of the coaxial cable, the return current of the circuit being located in an outer cylindrical can. In a bipolar battery, a circular cathode and anode are separated by a circular ionically conducting separator to form a cell. A number of these cells are then stacked together and separated by cylindrical electronic conductors, such as 
steel plates, to form the battery, which resembles the Volta pile. Although a cylindrical wire is a reasonable approximation of the bipolar battery geometry, it does not account for the details of the battery. The solid wire obviously lacks the multiple regions of various conductivities, surfaces of capacitance, electromotive force, and chemical reactions, and possible concentration variations found in a battery. However, as a first approximation, the infinite-wire analysis will allow us to predict the rate and the manner in which an electric field penetrates a conductor. And although this falls well short of a complete analysis, a considerable amount of information can still be inferred about the magnetic field effects.

Solutions have been derived for the electric field distribution inside and outside of an infinite wire maintained at a fixed current. A. Marcus ${ }^{11}$ provided the field distribution of the infinite wire surrounded by an axially symmetric, perfectly conducting, outer cylinder. Sommerfeld ${ }^{12}$ described the field distribution of a steady current in an infinite wire in free space. And D. Marcuse ${ }^{13}$ gave the general solution to the electric field distribution of an infinite wire carrying a steady current; the particular solution being a function of the rest of the circuit configuration.

Solutions to the steady-periodic electric field distributions in media of high but finite conductivity also exist. Maxwel1 14 solved for the self inductance of a periodic electric field in a cylindrical conductor. Jackson ${ }^{15}$ showed the electric field distribution of a periodic electric field penetrating a slab. 
The problem that has been solved that closest resembles the problem we address here is that of a linearly increasing current in an infinite conductor. This problem was addressed by Shakur ${ }^{16}$ and furthered by thervenak. ${ }^{17}$ However, these analyses assume that the current is uniform through the conductor; Heald ${ }^{2}$ raised some further questions of the results.

Typically, when solving the Maxwell equations in electrically conducting media, the first term on the right of equations 1 and 2 , referred to as the "displacement current," is omitted. $6,7,18,19,20$ In this analysis, this term is retained in what we shall call the "full solution" and in the "inner solution" and neglected in the "diffusion solution." The development of these solutions will elucidate the effect the displacement current plays in the initial stages of the field penetration and provide criteria for cases when its omission is justified, greatly simplifying the equations.

The full solution maintains all the physics and is applicable for all times; however its open form requires a large number of terms to investigate the short time regime. To probe the short time effects the problem is divided into the three regimes depicted in figure 1. Region $I$ is the inner regime. The solution obtained for this regime highlights the effects of the initial penetration of the electric field traveling at the speed of light into the conductor. Region II is the diffusion regime. After a time of $(0 \epsilon / \sigma)$ the field penetrates parabolically into the conductor. Region III is the outer regime. This is a long time regime in which the field penetrates to 


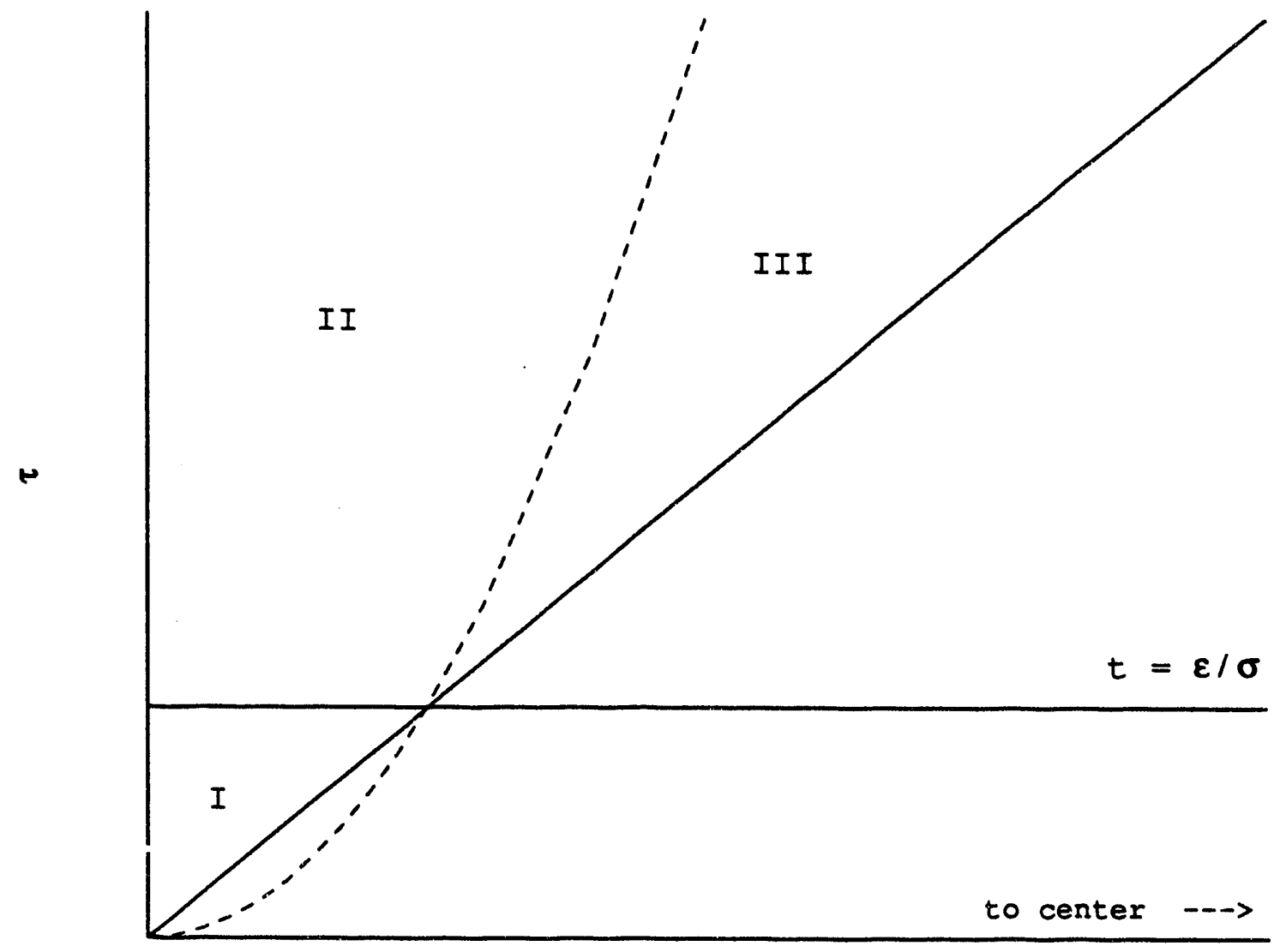

$1-\xi$

Figure 1. Solution regimes in the space-time coordinate. 
the center of the conductor at the speed of light, but with an essentially negligible magnitude. We shall not refer to this regime again.

\section{Full Solution}

For the geometry described above, the characteristic length is the radius of the inner conductor, $r_{0}$. Defining the electric diffusivity of the propagating electric field as the reciprocal of the electric conductivity times the permes ility, $1 / \mu \sigma$, a characteristic time is developed as the radius of the wire squared divided by the electric diffusivity. Thus, substitution of the dimensionless parameters

$$
\xi=r_{1} r_{0} \text { and } \tau-t / r_{0}^{2} \mu \sigma
$$

into equation 1 gives it the form

$$
\frac{\partial^{2} E}{\partial \xi^{2}}+\frac{1 \partial E}{\xi \partial \xi}=\delta^{2} \frac{\partial^{2} E}{\partial \tau^{2}}+\frac{\partial E}{\partial \tau},
$$

where $\delta=1 / \mu \sigma r_{0} \sqrt{\mu \epsilon} . \quad \delta$ can be interpreted as the ratio of the displacement current to the conduction current. To simplify matters, we assume that initially the electric field is zero in the wire; then, at time 0 , a step change in the electric field with axial component of magnitude $E_{0}$ at a certain position $z$ and $r_{0}$ is imposed. This signal, as it proceeds along the coaxial line, will be felt initially only on the inner wall of the outer conductor and outer wall of the inner conductor. If the outer conductor is a perfect conductor, as 
in this example, the rate of propagation of the electric field throughout the inner conductor will be the limiting effect to steady state. Thus, in this investigation we shall treat only the propagation of the field into the inner conductor. The conditions are described in mathematical terms as:

bounlary conditions

$$
\begin{aligned}
& E \text { is well-behaved at } \xi=0 \text {; } \\
& E=E_{0} \text { at } \xi=1 \text {; }
\end{aligned}
$$

initial conditions

$$
\begin{aligned}
& E=0 \quad \text { at } \quad \tau=0 \\
& \frac{\partial E}{\partial \tau}=0 \quad \text { at } \quad \tau=0 .
\end{aligned}
$$

The Laplace transformed solution of equation 3 is

$$
\frac{\bar{E}(s)}{E_{0}}=\frac{I_{0}\left[\left(\delta^{2} s^{2}+s\right)^{\frac{1}{2}} \xi\right]}{s I_{0}\left[\left(\delta^{2} s^{2}+s\right)^{3 / 2}\right]},
$$

where $I_{p}(x)$ is the modified Bessel function of the first kind, of order $p$. This solution can then be inverted by the method of residues.

Regardless of the solution scheme used, the radial penetration of the axial electric field component is given by ${ }^{*}$

When $2 \lambda_{k} \delta>1, \omega_{k}$ becomes a pure imaginary number. Equation 5 continues to apply. 
$\frac{E}{E_{0}}=\sum_{k=1}^{\infty} \frac{2 J_{o}\left(\lambda_{k} \xi\right)}{\lambda_{k} J_{1}\left(\lambda_{k}\right)}\left\{1-\left(\cosh \left(\omega_{k} \tau\right)+\frac{\sinh \left(\omega_{k} \tau\right)}{2 \delta^{2} \omega_{k}}\right) \exp \left(-\tau / 2 \delta^{2}\right)\right\}$,

where $\omega_{k}=\left[1-\left(2 \lambda_{k} \delta\right)^{2}\right]^{\frac{1}{2}} / 2 \delta^{2}, J_{p}(x)$ is the Bessel function of the first kind of order $p$, and $\lambda_{k}$ is the $k^{\text {th }}$ zero of $J_{0}(x)$. Although this solution is mathematically correct, it is not of a beneficial form for investigating short-time results. We say this because more and more terms of the summation are required for an accurate numerical solution at shorter and shorter times. With this in mind, we shall reapproach the problem using an asymptotic solution scheme to develop a long-time and a short-time solution. As will be shown, the long-time or diffusion solution is that which is felt throughout the conductor for times proportional to $r_{0}^{2} \mu \sigma$ (for a $10-\mathrm{cm}$-thick lead wire this is $\approx 60 \mathrm{~ms}$ ), whereas, the short-time or inner solution is that which is felt just at the outer edge of the conductor at a time scale proportional to $\epsilon / \sigma$ (for the same lead wire this is $\approx 1 \times 10^{-18} \mathrm{~s}$ ). This approach will allow us to describe the development of the electric field for all time regimes. Since the first term on the right of equation 3 is usually neglected, $\delta$ was chosen as the stretching parameter.

\section{Diffusion Solution}

The variables in equation 3 have been made dimensionless such that they are of $O(1)$ in the diffusion region. It follows that, as a first approximation, the first term on the right of equation 3 can be neglected for $\delta^{2} \ll 1$. The equation reduces to the form of the 
transient diffusion equation in cylindrical coordinates found in the mass-transfer literature,

$$
\frac{\partial^{2} E}{\partial \xi^{2}}+\frac{1}{\xi} \frac{\partial E}{\partial \xi}=\frac{\partial E}{\partial \tau} .
$$

This is a major simplification; the highest derivative with respect to time is omitted. However, the eçuation takes a more manageable form, and one that we are familiar solving. We shall address the implications of this mathematical simplification, shortly.

Equation 6 is solved by separation of variables with the boundary and first of the initial conditions listed above equation 4 to yield

$$
\frac{E}{E_{0}}=\sum_{k=1}^{\infty} \frac{2 J_{o}\left(\lambda_{k} \xi\right)}{\lambda_{k} J_{1}\left(\lambda_{k}\right)}\left\{1-\exp \left(-\lambda_{k}^{2} r\right)\right\} .
$$

Equation 7 is plotted as a function of $\xi$ for different fractions of the time constant in figure 2. The current distribution is directly related to the electric field distribution through Ohm's law, $i=\sigma E$. Other than the absence of the hyperbolic cosines and sines and $\omega^{\prime} s$ of the full solution, this solution does not appear to be much of an improvement over the "full solution", equation 5, since it too is of open form. Nevertheless, from this solution it is apparent that the time constant for the exponential decay of the long-time solution is

$$
\tau_{0}=\frac{r_{o}^{2} \mu \sigma}{\lambda_{1}^{2}}
$$




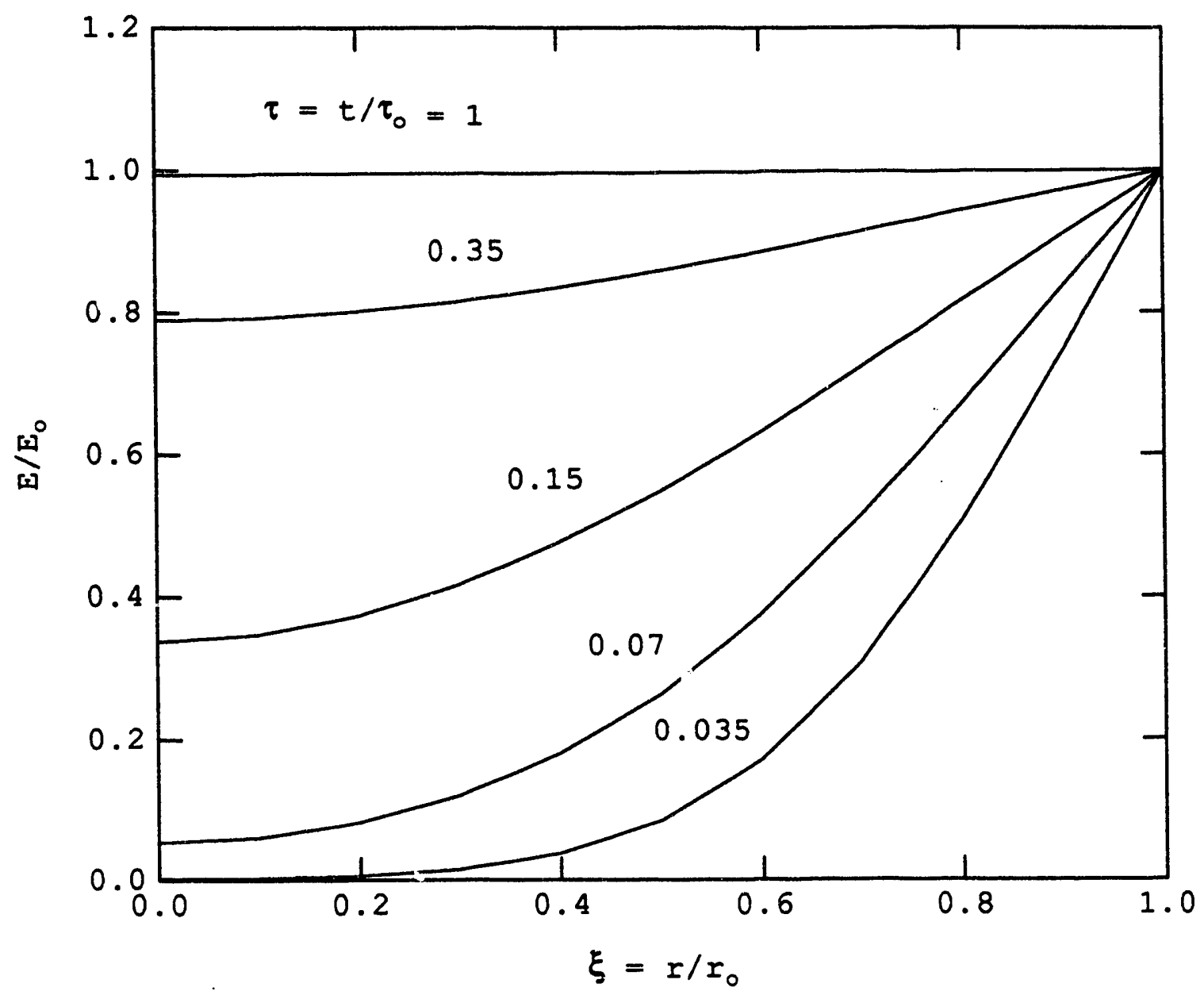

Figure 2. Axial electric field distibution divided by the magnitude of the electric field at the surface versus the dimensionless distance from the center of a cylindrical conductor. 
This is the same time regime that is approximated by a the lumpedinductance analysis where the current approaches the steady-state current exponentially as the time over the inductive time constant $I / R$. Thus, $I / R$ is equal to $\tau_{0}$.

We now solve for the short-time solution of the diffusion region to determine how the field increases with time for relatively short times (and for future comparison with the long-time solution of the inner region.) Solving equation 6 by Laplace transforms,

$$
\frac{\bar{E}(s)}{E_{0}}=\frac{I_{0}\left(s^{\frac{1}{2}} \xi\right)}{s I_{0}\left(s^{\frac{1}{2}}\right)}
$$

expanding this solution for large $s$, and then inverting it, we obtain the short-time solution of the diffusion region,

$$
\frac{E}{E_{0}}=\frac{1}{\xi^{\frac{3}{2}}}\left[1-\frac{9 \tau}{32 \xi^{2}}+\frac{3675 \tau^{2}}{2048 \xi^{4}}-o\left(\frac{\tau^{3}}{\xi^{6}}\right)\right] \operatorname{erfc} \frac{1-\xi}{\sqrt{4 \tau}}
$$

where $\tau \ll 1$. One should note that fewer terms of this solution are needed as $\xi \rightarrow 1$ and $\tau \rightarrow 0$. Thus, at short times the electric field penetrates as the erfc of distance from the edge of the conductor divided by the square root of time. We thus define a penetration depth, which is analogous to the skin-depth of a sinusoidal signal, as

$$
d_{p}=\sqrt{t / \mu \sigma}
$$

The solutions developed in this, the diffusion regime, contain all the physics of equation 1 that occur on the time frame of $\tau_{0}$. We 
should now like to investigate the consequences of dropping the second order time derivative that provided these results and to elucidate the physics that occur immediately following the application of the electric field.

\section{Inner Solution}

To obtain the short-time or inner solution, we shall first define the variable $\xi^{\prime}=1-\xi$, a dimensionless distance from the edge of the conductor, and then define new independent variables by stretching $\xi^{\prime}$ and $\tau$ by powers of $\delta$. That is, let

$$
\bar{\xi}=\frac{1-\xi}{\delta^{n}} \text { and } \quad \bar{\tau}=\frac{\tau}{\delta^{m}}
$$

Setting $m=2$ and $n=1$, substituting them into equation 3 , and keeping terms multiplied $b$; the smallest powers of $\delta$, we obtain the equation

$$
\frac{\partial^{2} E}{\partial \bar{\xi}^{2}}=\frac{\partial^{2} E}{\partial \bar{\tau}^{2}}+\frac{\partial E}{\partial \bar{\tau}},
$$

with boundary conditions,

$$
\begin{aligned}
& E=E_{0}, \quad \text { at } \bar{\xi}=0 ; \\
& E \text { is well behaved, as } \bar{\xi} \rightarrow \infty ;
\end{aligned}
$$

and initial conditions,

$$
\begin{aligned}
& E=0, \quad \text { at } \bar{\tau}=0 ; \\
& \frac{\partial E}{\partial \bar{\tau}}=0, \quad \text { at } \bar{\tau}=0 .
\end{aligned}
$$

(Note the absence of the $1 / \xi$ term on the left of equation 12, implying that the curvature of the wire has little effect in this time 
regime.) These equations were solved by the Laplace-transform technique to give

$$
\bar{E}(s)=E_{0} \frac{\exp \left[-\left(s^{2}+s\right)^{\frac{1}{2}} \bar{\xi}\right]}{s} .
$$

Applying the complex inversion formula to this solution while integrating around the branch cut between 0 and -1 , we get the solution

$\div$

$$
E=E_{0}\left(1-\frac{1}{\pi} \int_{0}^{1} \frac{e^{-x \bar{r}} \sin [\sqrt{|x|[1-x]} \bar{\xi}]}{x} d x\right) u(\bar{r}-\bar{\xi})
$$

where $u(\bar{r}-\bar{\xi})$ is the Heaviside function. 21 We extract more information from this time regime by expanding equation 13 for large and small values of $s$ to obtain the short and long time solutions, respectively. Expanding 13 for large values of $s$ and taking the inverse Laplace transform to get the short-time solution of the inner region gives

$$
\begin{aligned}
& E=E_{0} e^{-\bar{\xi} / 2}\left(1+\frac{\bar{\xi}}{8}(\bar{T}-\bar{\xi})-\frac{\bar{\xi}(8-\bar{\xi})}{256}(\bar{\tau}-\bar{\xi})^{2}+\frac{\bar{\xi}\left(120-24 \bar{\xi}+\bar{\xi}^{2}\right)}{18432}(\bar{\tau}-\bar{\xi})^{3}\right. \\
& \left.+o\left(\bar{\xi} \bar{T}^{4}\right)\right) u(\bar{\tau}-\bar{\xi}) .
\end{aligned}
$$

This solution indicates that the electric field initially penetrates the conductor essentially as a front, traveling at the speed of light, with a magnitude that is exponentially damped with distance from the surface. The characteristic length of penetration is $2 r_{0} \delta$. 
Beyond the distance of $\bar{\tau}=\bar{\xi}$ the field is zero.

Allowing $s$ to approach 0 in equation 13 and inverting to obtain the long-time solution of the inner region gives

$$
\frac{E}{E_{0}}=\operatorname{erfc}\left(\frac{\bar{\xi}}{(4 \bar{\tau})^{\frac{3}{2}}}\right)
$$

Thus after an initial period of $\epsilon / \sigma$ the field penetrates as the erfc of the distance divided by the square root of time. This is of the same form as the solution obtained as the short-time solution in the diffusion region, equation 11.

To summarize, when an electric field with an axial component of magnitude $E_{0}$ is applied to the surface of a conductor at time 0 , the field penetrates the conductor at the speed of light with a magnitude that is dramatically damped within in a distance of $\delta r_{0}$. This occurs within the time $0+$ and $\delta^{2} r_{0}^{2} \mu \sigma=\epsilon / \sigma$. This is a very short time frame. For comparison, the time it takes light to reach the center of the conductor is $\delta r_{0}^{2} \mu \sigma$. After this initial period, the field penetrates the conductor as the erfc of the distance from the surface divided by the square root of four times the time divided by $r_{0}^{2} \mu \sigma$. Finally, as $t$ approaches $r_{0}^{2} \mu \sigma$, the field approaches the uniform, steady-state field distribution.

From this analysis, we shall develop the solutions of the total current as a function of time. 


\section{Current}

From the expressions given for the electric field, one can determine the instantaneous total current. This value is derived from Ohm's law and integration of $i$ over the cross-sectional area,

$$
I=2 \pi r_{0}^{2} \sigma \int_{0}^{1} E \xi d \xi
$$

Solving for the instantaneous current allows us to compare the results of the above cases more readily by eliminating the independent variable $\xi$. The instantaneous current for the full solution and diffusion solution are, accordingly,

Full $\frac{I}{\pi r_{0}^{2} \sigma E_{0}}=1-\sum_{k=1}^{\infty} \frac{4}{\lambda_{k}^{2}}\left(\cosh \left(\omega_{k} \tau\right)+\frac{\sinh \left(\omega_{k} \tau\right)}{2 \delta^{2} \omega_{k}}\right) \exp \left(-\frac{\tau}{2 \delta^{2}}\right)$,

Diffusion

$$
\frac{I}{\pi r_{0}^{2} \sigma E_{0}}=1-\sum_{k=1}^{\infty} \frac{4}{\lambda_{k}^{2}} \exp \left(-\lambda_{k}^{2} \tau\right)
$$

Another approach to the full solution is first to integrate the Laplace transform of the electric field, equation 4, with respect to $\xi$ from 0 to 1 . Using the method of residues, we obtain the above solution; but expanding this equation for large $s$ and inverting gives a solution useful at short times,

$$
\begin{aligned}
\frac{I}{\pi r_{0}^{2} \sigma E_{0}} & =\frac{2 \tau e^{-\tau / 2 \delta^{2}}}{\delta}\left[I_{0}\left(\tau / 2 \delta^{2}\right)+I_{1}\left(\tau / 2 \delta^{2}\right)\right] \\
& -\left[\tau-\delta^{2}\left(1-e^{-\tau / \delta^{2}}\right)\right]-\frac{\tau^{2} e^{-\tau / 2 \delta^{2}}}{6 \delta}\left[I_{1}\left(\tau / 2 \delta^{2}\right)+I_{2}\left(\tau / 2 \delta^{2}\right)\right]
\end{aligned}
$$




$$
\begin{aligned}
& -\frac{1}{8}\left[\tau^{2}-2 \delta^{2} \tau\left(2+e^{-\tau / \delta^{2}}\right)+6 \delta^{4}\left(1-e^{-\tau / \delta^{2}}\right)\right] \\
& -\frac{5 \tau^{3} e^{-\tau / 2 \delta^{2}}}{48 \delta}\left[I_{2}\left(\tau / 2 \delta^{2}\right)+I_{3}\left(\tau / 2 \delta^{2}\right)\right]+O\left(\tau^{3}\right)
\end{aligned}
$$

for $r \ll 1$. Allowing $s$ to get very large provides the very-shorttime solution,

$$
\frac{I}{\pi r_{0}^{2} \sigma E_{0}}=2 \pi / \delta
$$

In the diffusion region, if we start with the transformed solution of the electric field, equation 10, integrate over the crosssectional area of the conductor, take the limit as $s$ gets very large, and invert it, we get the short-time solution to the diffusion region,

$$
\frac{I}{\pi r_{0}^{2} \sigma E_{0}}=\left(\frac{2 r}{\pi}\right)^{\frac{1}{2}}
$$

For the inner region, we shall first integrate equation 13 with respect to $\bar{\xi}$ from 0 to $\infty$ and then take the inverse Laplace transform. Here, the current is given as

$$
\text { Inner } \frac{I}{\pi r_{0}^{2} \sigma E_{0}}=2 \delta \bar{\tau} e^{-\bar{\tau} / 2}\left[I_{0}(\bar{\tau} / 2)+I_{1}(\bar{\tau} / 2)\right] \text {. }
$$

Since $\bar{\tau}-r / \delta^{2}$, we see that the first term of the expanded full solution, equation 20 , is equivalent to the short-time solution given here. The rest of the terms of equation 20 are corrective terms for large, which tend to bend the solution down to the long-time 
solution. If we take the limit of the Laplace transformed solution of the inner region, equation 13 , for very small $s$, and again integrate with respect to $\bar{\xi}$ from 0 to $\infty$, we get the long-time solution of the inner region,

$$
\frac{I}{\pi r_{0}^{2} \sigma E_{0}}-\delta\left(\frac{2 \bar{\tau}}{\pi}\right)^{1 / 2},
$$

and for very large $s$, we get the short-time solution of the inner region,

$$
\frac{I}{\pi r_{0}^{2} \sigma E_{0}}=2 \delta \bar{\tau}
$$

From this analysis of the current distribution, we see that at short times $(t<\epsilon / \sigma)$ the current is proportional to time to the first power and at long times $(t>\epsilon / \sigma)$ is proportional to time to the one half power until $t$ is of order $r_{0}^{2} \mu \sigma$ where it approaches the steadystate value (see figure 3). In the former case, the short-time solution of the inner region overlaps the very-short-time solution of the full solution. In the latter case, the long-time solution of the inner region overlaps the short-time solution of the diffusion region. We can conclude that the "displacement current," which is responsible for this difference in solutions, is important only for $\bar{\tau}=r / \delta^{2} \leq o(1)$ where $\delta^{2} \ll 1$. One further note, figure 2 shows that the current approaches the final steady-state asymptotically with an exponential time constant of $r_{0}^{2} \mu \sigma$. This is as predicted by the lumped-inductance analysis - the only thing in this work predicted 


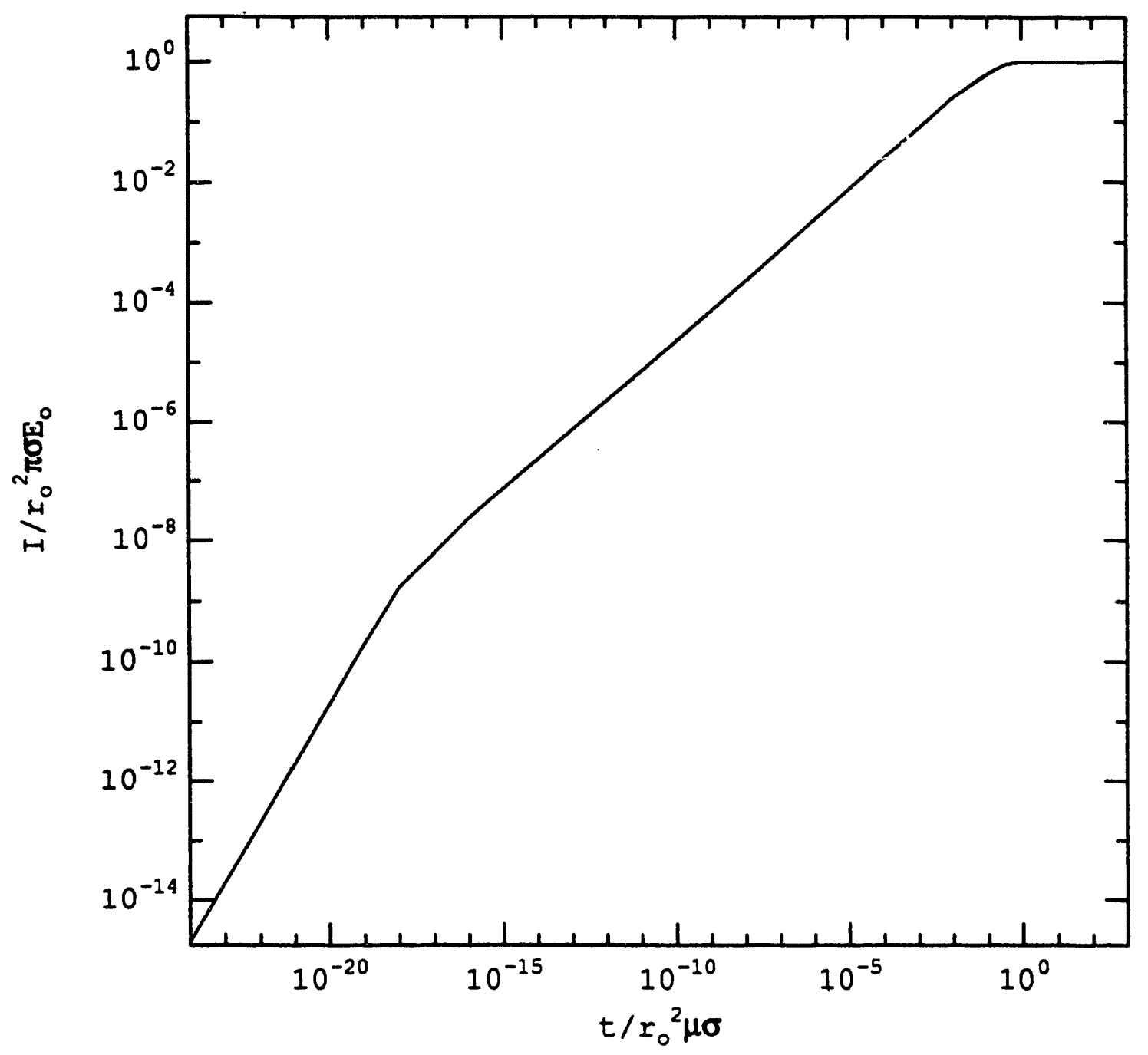

Figure 3. Dimensionless current versus dimensionless time for $\delta=1 \times 10^{-9}$. 
by the lumped-inductance analysis.

\section{Summary}

This analysis describes the penetration of a steadily applied electric field into a cylindrical conductor from the initial time to steady state. This is recognized as a crude first approximation to the current distribution in a bipolar battery. The solution scheme used introduced two distinct time regimes referred to as the inner and diffusion solutions. A long-time and short-time solution of the electric field distribution were determined in each regime and then confirmed as consistent by direct comparison and comparison of the instantaneous current distributions. The current initially penetrates the wire at a rate proportional to time up to a time of the order of $\epsilon / \sigma$ and then at a rate proportional to the square root of time. It is this same order of time after which the omission of the displacement current is justified. The lumped inductance analysis is appropriate for times on the order of $r_{0}^{2} \mu \sigma$.

It part II we shall include the cylindrical wire in a radial circuit. That analysis will describe the interactions of the fields in the conductor with the rest of the circuit in terms of the time constant of the complete system.

(It should be noted that all calculations in this paper have been performed assuming that $\sigma, \epsilon$, and $\mu$ are independent of time; i.e., the conductor is nondispersive.) 


\section{List of Symbols}

Roman

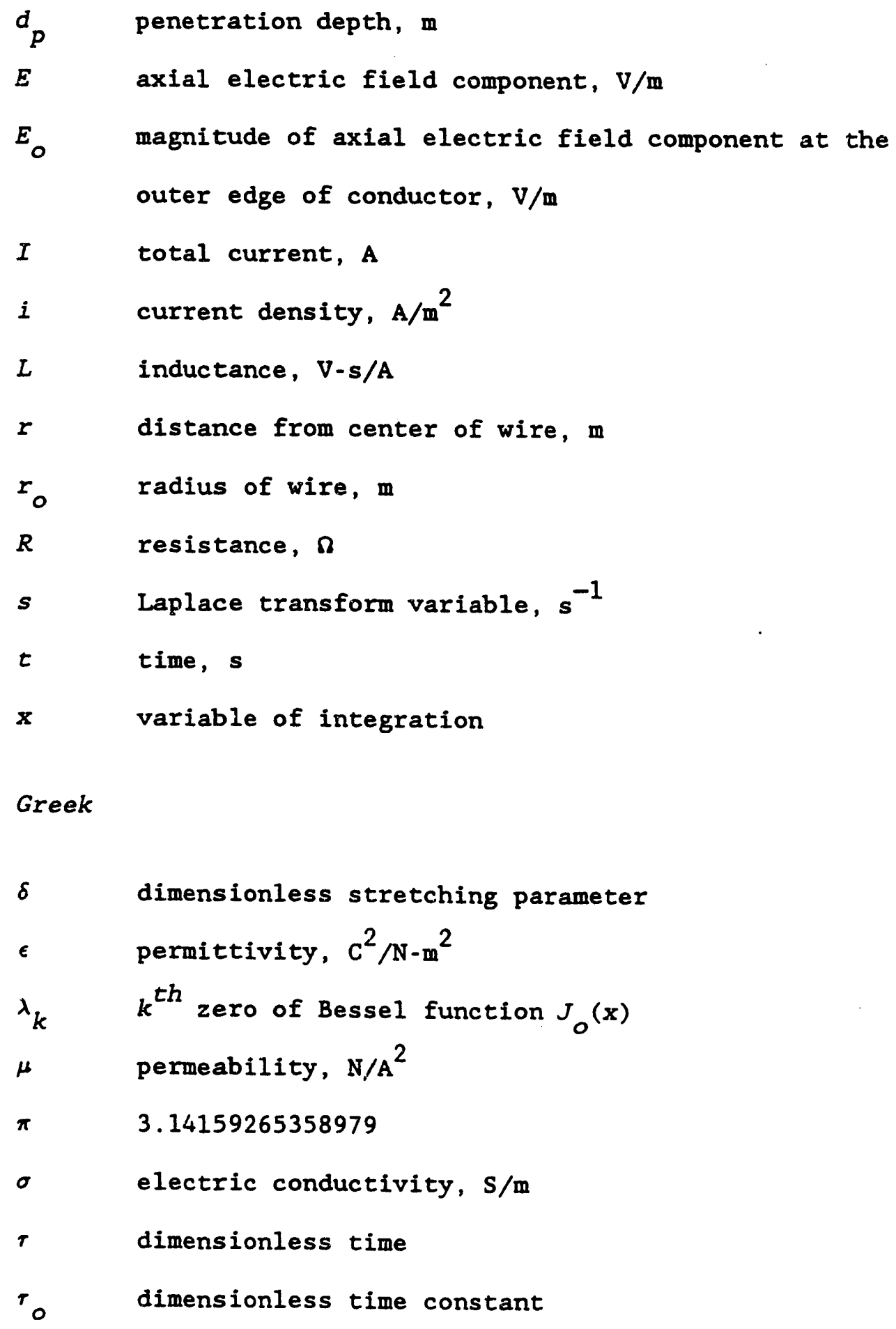




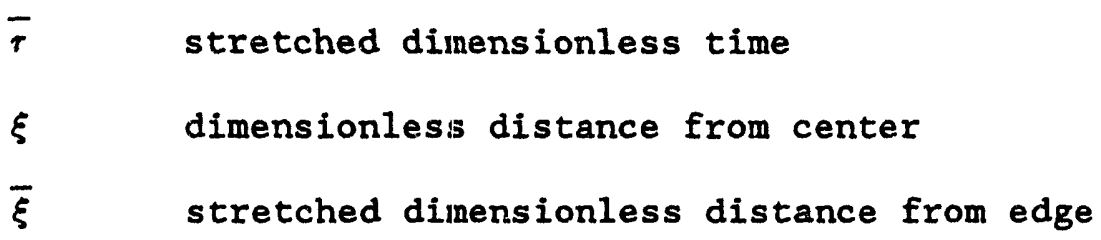

References

1. P. L. Kapitza, "A Method of Producing Strong Magnetic Fields," Proc. Roy. Soc. Lond., 105A, 691-710 (1924).

2. E. Willihnganz, "The Voltage of the Sponge Lead Plate During Discharge of the Storage Battery," Trans. Electrochem. Soc., 79, $243-251$ (1941).

3. H. F. Gibbard, "High Temperature, High Pulse Power Lithium Batteries," Journal of Power Sources, 26, 81-91 (1989).

4. H. F. Gibbard, "Ultra-High-Power Batteries," Electrochemical Society Proc., 86-12, 193-205 (1986).

5. R. M. LaFollette and D. N. Bennion, "Design Fundamentals of High Power Density, Pulsed Discharge, Lead Acid Batteries: I Experimental," J. Electrochem. Soc., 137, 3693-3701 (1990).

6. R. M. LaFollette and D. N. Bennion, "Design Fundamentals of High Power Density, Pulsed Discharge, Lead Acid Batteries: II Modeling," J. Electrochem. Soc., 137, 3701-3707 (1990). 
7. B. McKinney, W. Tiedemann, and J. Newman, "Short Circuit Currents in Uninterruptible Power Source Batteries," Proceedings of the Symposium on Advances in Lead-Acid Batteries, Pennington N. J., $3336-3347$ (1984).

8. B. D. Cahan, M. L. Daroux, and E. B. Yeager, "Effect of Physical and Geometrical Factors on the Impedance of Electrochemical Power Sources," Proc. - Electrochemical Soc., 87-12, 137-151 (1987).

9. W. G. V. Rosser, "What Makes an Electric Current Flow," Am. J. Phys., 31, 884 (1963).

10. M. A. Heald, "Electric Fields and Charges in Elementary Circuits," Am. J. Phys., 52, 522-526 (1984).

11. A. Marcus, "The Electric Field Associated with a steady Current in Long Cylindrical Conductor," Am. J. Phys., 9, 225-226 (1941).

12. A. Sommerfeld, Electrodynamics, Academic Press, New York, $125-133(1952)$.

13. D. Marcuse, "Comment on 'The Electric Field Outside a Long Straight wire Carrying a Steady Current," " Am. J. Phys., 38, 935-936 (1970).

14. J. C. Maxwe11, A Treatise on Electricity and Magnetism, 3rd Edition, Dover Publications, Inc., New York (1954). 
15. J. D. Jackson, Classical Electrodynamics, 2nd Edition, John Wiley \& Sons, New York (1975).

16. M. A. Shakur, "Solution to the Problem on Page 785," Am. J. Phys., 52, 849 (1984).

17. J. G. Chervenak, "The Back Electric Field from a Long Wire," Am. J. Phys., 54, $946-947$ (1986).

18. P. Silvester, "AC Resistance and Reactance of Isolated Rectangular Conductors," IEEE Transactions on Power Apparatus and Systems, 86, 770-774 (1967).

19. J. R. Carson, "Wave Propagation over Parallel Wires: The Proximity Effect." Phil. Mag., 41, 607-633 (1921).

20. J. R. Gosselin, P. Rochon, and N. Gauthier, "Study of Eddy Currents in a Cylindrical Wire: An Undergraduate Laboratory Experiment," Am. J. Phys., 50, $440-443$ (1982).

21. M. Abramowitz and I. A. Stegun, Handbook of Mathematical Functions, Dover Publications, Inc., New York (1965). 


\section{Chapter 2}

Magnetic Field Effects in High-Power Batteries

Part II: Time Constant of a Radial Circuit Terminated by a Cylindrical Cell with Inductance

Foreword

The time constant to steady-state of an electric signal applied to the outer boundary of a radial circuit that propagates to a centrally located cylindrical conductor is determined rigorously by a transmission-line analysis. Two cases are reported: the perfect radial conductor and a radial conductor with a finite resistance. The time constant is featured as a function of the ratio of the radius of the radial conductive leads to the radius of the inner conductor. As the ratio is increased, the time constant departs from that of an electric field penetrating a cylindrical conductor and approaches the inductive time constant of the leads. An analytic approximation of the time constant of the system is also provided. This analysis should assist in the development of high-power circuitry design when the discharge time is on the order of the inductive time constant.

\section{Introduction}

The intent of this research is to determine the inductive time constant of a circuit that contains a battery. As discussed by 
Rosser, ${ }^{1}$ the rate at which the current rises in one portion of a circuit is a function of the configuration and properties of the rest of the circuit. Therefore, this problem cannot be solved in parts. The rigorous method for handling the inductance effects is to solve Maxwell's equations of electromagnetism throughout the entire circuit and surrounding medium, simultaneously with the equations that govern battery performance. This would require an enormous effort. As such, we direct our investigation to the simpler case of determining rigorously the rate of discharge of a radial circuit with an inductive, coaxial, cylindrical core. Applying the same arguments as found in part I, the cylindrical core is assumed to approximate the behavior of a bipolar battery. Furthermore, the electromotive force is assumed rooted at some radial distance, $r_{0}$, away.

In part I we developed the solution to the rate at which an electric field penetrates a cylindrical conductor. As discussed, a solid conductor is a poor approximation of the inner workings of a battery. However, that analysis provided an adequate first approximation of the inductive behavior of the battery. In this section, we shall use a transmission-line analysis in conjunction with the preceding work to determine the time constant of the total circuit configuration. The solution to this full problem may suggest where the limitation to instantaneous discharge resides and may then be used for design criteria for systems intended to be discharged at high rates. 


\section{Analytic Approach}

An analytic approach is possible if a simplified circuit geometry is proposed. The following configuration is considered: a cylindrical conductor of finite length $d$, conductivity $\sigma_{i}$, and radius $r_{i}$ sandwiched between two circular plates of radius $r_{0}$, where $r_{0}>r_{i}$ (see figure 1.) The system is initially at open-circuit, and the potential is zero everywhere. A constant voltage source is then applied at time zero at $r=r_{0}$. We wish to determine the time it takes the system to reach steady-state. A transmission-line analysis shall be used to characterize the propagation of the signal along the radial conductors to the inner cylindrical conductor. The solution derived in part I section for the penetration of an electric field into a conductor shall be converted into a boundary condition at $r=r_{i}$. The problem is solved using Laplace transforms. Two cases are considered: the first is where the outer radial conductors are assumed to have an infinite conductivity; and the second is where they have a finite conductivity. Before proceeding to the solution, we shall first develop, through the elementary laws of statics and electrodynamics, the transmission-line equations.

\section{Transmission-Line Analysis}

A measure of the ease by which charge $q$ migrates through a medium is characterized by its resistivity, $\rho$. This parameter is lowest for conductors, of moderate value for semiconductors, and highest for insulators. The total resistance of a bar of a conducting material of length $I$ and area $A$ is 


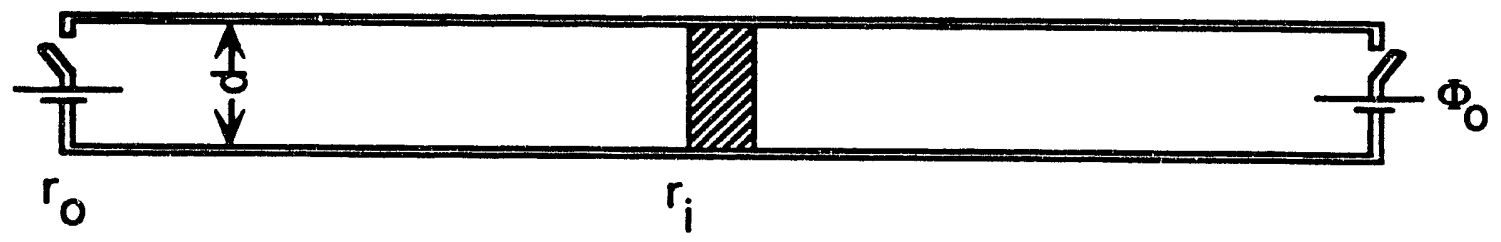

Figure 1. Radial circuit of radius $r_{0}$ containing a cylindrical conductor of radius $r_{i}$. 


$$
R=\frac{\rho I}{A} \text {. }
$$

Thus the resistance is defined by two parameters: the physical geometry and resistivity.

According to Gauss's law, an electric field outside of a conductor acting perpendicular to the conductor is given by

$$
\mathbf{E} \cdot \boldsymbol{n}=\frac{q}{\epsilon A} .
$$

The potential is defined as

$$
-\nabla \Phi-\mathbf{E}
$$

For two parallel plates of equal and opposite charge and distance $d$ apart we obtain

$$
\Phi=\frac{g d}{\epsilon A}
$$

or

$$
q=C \Phi,
$$

where $C$ is the capacitance. The capacitance, like the resistance, is a function of the physical geometry and a parameter, $\epsilon$, that describes the medium separating the conductive material. For the parallel plates, capacitance per unit area is defined as

$$
c=\frac{C}{A}=\epsilon / d \text {. }
$$

Current is defined as the amount of charge that passes a particular point per unit of time. In differential form

$$
I=\frac{\partial q}{\partial t}=C \frac{\partial \Phi}{\partial t}
$$

for a capacitive current. 
Ampere's law shows that with the passing of any current there is an associated magnetic field,

$$
\nabla \times B=\mu I .
$$

For a current flowing in a wire, the magnetic field is proportional to the amount of current and inversely proportional to the square of the distance from the center of the wire,

$$
B \propto \mu I_{L} / r^{2} .
$$

The magnetic flux, $\phi$, is the sum of the magnetic fields of currentcarrying elements from different positions in the conductor. The magnetic linkage, $\lambda$, is the sum of the total magnetic flux in the system. The magnetic linkage is proportional to the current,

$$
\lambda=L I_{L}
$$

$L$, the self-inductance, is a proportionality constant between the flux linkage and the current and again is a function of the physical geometry and $\mu$, a parameter describing the surrounding medium. For a parallel-plate configuration, it can be shown that the selfinductance is

$$
L=\mu d \text {. }
$$

To relate the flux linkage back to a potential, we turn to Faraday's law,

$$
\nabla \times \mathbf{E}--\frac{\partial \mathbf{B}}{\partial t}
$$

which mathematically states that a magnetic field that is varying in time has associated with it an electric field. These definitions 
ultimately lead to the expression

$$
-\nabla \Phi=L \frac{\partial i_{L}}{\partial t}
$$

We can now discuss the transmission of a signal in the radial direction between two conducting plates. If the conductors are "perfect," i.e. have a resistivity of zero, charge travels between the plates on the surfaces and is referred to as the skin current. If the plates have a finite resistance, the current penetrates within the plates and it can be integrated over the thickness $l$. In either case, the current density will have units of $\mathrm{A} / \mathrm{cm}$. Charge flowing in the radial direction behaves as a purely inductive current, $i_{L}$, if the plates are perfect, and will contain an ohmic term if they are not. Some of the charge goes to charging the plates and is referred to as the capacitive current, $i_{c}$, with units of $\mathrm{A} / \mathrm{cm}^{2}$. A shell balance of the current traveling in the radial direction along a plate with no resistance entering the shell at $r$ and leaving at $r+\Delta r$ appears as

$$
\left.2 \pi r i_{L}\right|_{r}=\left.2 \pi r i_{L}\right|_{r}+\Delta r+2 \pi r \Delta r i_{C} .
$$

Dividing through by $r \Delta r$ and taking the limit as $\Delta r$ approaches zero gives the differential form

$$
\frac{1}{r} \frac{\partial\left(r i_{L}\right)}{\partial r}=-i_{c}
$$

Differentiation of equation 15 with respect to time and the subsequent substitution of the previously derived current-potential relationships, 


$$
\frac{\partial \Phi}{\partial r}=-L \frac{\partial i_{L}}{\partial t}
$$

and

$$
i_{c}=c \frac{\partial \Phi}{\partial t}
$$

gives the transmission-line equation for a radial circuit of infinite conductivity,

$$
\frac{1}{r} \frac{\partial}{\partial r}\left(r \frac{\partial \Phi}{\partial r}\right)=L c \frac{\partial^{2} \Phi}{\partial t^{2}} .
$$

If the conductor maintains a finite resistivity, the equation

$$
-\frac{\partial \Phi}{\partial r}=L \frac{\partial i_{L}}{\partial t}+\frac{i_{L}}{\sigma I}
$$

( $\sigma$, the conductivity, is equal to $1 / \rho^{\text {.) }}$ which contains an additional term for the ohmic drop, is substituted into equation 15 in the place of equation 16. This substitution gives the transmission-line equation for a radial circuit with finite conductivity,

$$
\begin{aligned}
& \qquad \begin{array}{l}
\frac{1}{r} \frac{\partial}{\partial r}\left(r \frac{\partial \Phi}{\partial r}\right)=L c \frac{\partial^{2} \Phi}{\partial t^{2}}+\frac{c}{\sigma l} \frac{\partial \Phi}{\partial t} \\
\text { Case } 1:
\end{array} \\
& \text { Outer Conductors of Infinite Conductivity. }
\end{aligned}
$$

As shown above, the following transmission-1ine equation for the potential applies to radial conductors of infinite conductivity:

$$
\frac{\partial^{2} \Phi}{\partial r^{2}}+\frac{1}{r} \frac{\partial \Phi}{\partial r}=L c \frac{\partial^{2} \Phi}{\partial t^{2}} .
$$

Substitution of the dimensionless variables, 


$$
\xi=r / r_{i}, \quad \tau=t / r_{i}^{2} \mu_{i} \sigma_{i}, \quad \text { and } \phi=\Phi / \Phi_{0},
$$

leads to the equation

$$
\frac{\partial^{2} \phi}{\partial \xi^{2}}+\frac{1}{\xi} \frac{\partial \phi}{\partial \xi}=\delta_{0}^{2} \frac{\partial^{2} \phi}{\partial \tau^{2}},
$$

where $\delta_{0}=r_{i} \sqrt{L c} / r_{i}^{2} \mu_{i} \sigma_{i}$.

We shall solve this equation by means of a Laplace transformation. The Laplace transform of equation 23 is

$$
\frac{\partial^{2} \bar{\phi}}{\partial \xi^{2}}+\frac{1}{\xi} \frac{\partial \bar{\phi}}{\partial \xi}=\delta_{0}^{2} s^{2} \bar{\phi}-s \phi(+0)-\phi^{\prime}(+0) .
$$

The following is a list of the initial and boundary conditions.

Initial Conditions

$$
\begin{aligned}
& \phi=0 \quad \text { at } \tau=0, \\
& \frac{\partial \phi}{\partial \tau}=0 \text { at } \tau=0 .
\end{aligned}
$$

The first condition mathematically states that the potential is zero everywhere at time zero. The second condition states that the change of the potential with respect to time at time zero is also zero. This implies that some type of "inertia" or inductance must be overcome before the potential will change at time zero.

Boundary Conditions

$$
\begin{aligned}
& \phi=1 \quad \text { at } \xi=r_{0} / r_{i}, \\
& 2 \pi \int i_{i} r d r=2 \pi r_{i} i_{0} \quad \text { at } \xi=1 .
\end{aligned}
$$

The first boundary condition states that at $\ddot{x}=r_{0}$ the potential is 
instantaneously set to $\Phi_{0^{\circ}}$. The second condition is interpreted as the integral of the current density over the area in the inner conductor is equal to the line integral of the skin-current density that enters the inner conductor at $r=r_{i}$.

We shall now derive the Laplace transformation of the boundary conditions. The Laplace transformation of equation 27 gives

$$
\bar{\phi}=1 / s \quad \text { at } \xi=r_{0} / r_{i}
$$

On the right side of equation $28, i_{0}$, the skin-current density, defined in equation 16 , is

$$
i_{0}=-\frac{1}{L} \int_{0}^{t} \frac{\partial \Phi}{\partial r} d t=-\frac{r_{i} \mu_{i} \sigma_{i} \Phi_{0}}{L} \int_{0}^{\tau} \frac{\partial \phi}{\partial \xi} d \tau .
$$

The Laplace transformation of this equation is

$$
\bar{i}_{0}=-\frac{r_{i} \mu_{i}{ }^{\sigma} \Phi_{0}}{L} \frac{1}{s} \frac{\partial \bar{\phi}}{\partial \xi} .
$$

Ohm's law gives the relation for current density in the central conductor in terms of a component of electric field

$$
i_{i}=\sigma_{i} E \text {. }
$$

We have shown in part I that inside a cylindrical conductor the Laplace transform of the axial component of the electric field distribution due to a steady field of unit magnitude applied at the surface is 


$$
\bar{E}=-\frac{\Phi_{0} \bar{\phi}}{d}=\frac{I_{0}\left[\left(\delta_{i}^{2} s^{2}+s\right)^{\frac{1}{2}} \xi\right]}{s I_{0}\left[\left(\delta_{i}^{2} s^{2}+s\right)^{3 / 2}\right]},
$$

$I_{0}$ is the modified Bessel function of the first kind, of order $0 .{ }^{2}$ Duhamel's superposition formula, ${ }^{2}$ an integration of the solution to the linear equations applicable to the system, is used to describe the current in the conductor

$$
i_{i}=\sigma \int_{0}^{\tau} E\left(\xi, \tau-\tau^{\prime}\right) \frac{\partial E\left(\tau^{\prime}\right)}{\partial \tau} d \tau^{\prime}
$$

The Laplace transform of this equation is equal to the product of the Laplace transform of its parts. ${ }^{2}$ The Laplace transform of $E\left(\xi, \tau-\tau^{\prime}\right)$ is given in equation 33. The Laplace transform of the derivative of $E$ with respect to $\tau$ is

$$
\frac{\overline{\partial E}}{\partial r}=s \bar{E} .
$$

Taking the Laplace transform of equation 34 and substituting into it equations 33 and 35 gives

$$
\frac{2 \pi r_{i}^{2} \sigma_{i} \Phi_{0} \bar{\phi}}{d} \int_{0}^{1} \frac{\xi I_{0}\left[\left(\delta_{i}^{2} s^{2}+s\right)^{3 / 2} \xi\right]}{I_{0}\left(\delta_{i}^{2} s^{2}+s\right)^{3 / 2}} d \xi=\frac{2 \pi r_{i}^{2} \mu_{i} \sigma_{i} \Phi_{0}}{L} \frac{1}{s} \frac{\partial \bar{\phi}}{\partial \xi}
$$

After integrating and some rearrangement, we get the boundary condition,

$$
\frac{\bar{\phi} I_{i}\left(\delta_{i}^{2} s^{2}+s\right)^{\frac{1 / 2}{2}}}{\left(\delta_{i}^{2} s^{2}+s\right)^{3 / 2} I_{0}\left(\delta^{2} s^{2}+s\right)^{3 / 2}}=\frac{\mu_{i}}{L d} \frac{1}{s} \frac{\partial \bar{\phi}}{\partial \xi} \quad \text { at } \quad \xi=1
$$




\section{Solution}

Solution of equation 24 with the conditions of $25,26,29$, and 37 is

$$
\begin{gathered}
\bar{\phi}=A I_{0}\left(\delta_{0} s \xi\right)+B K_{0}\left(\delta_{0} s \xi\right) . \\
A=\frac{\frac{1}{s}-B K_{0}\left(\delta s \frac{r_{0}}{r_{i}}\right)}{I_{0}\left(\delta \delta s \frac{r_{0}}{r_{i}}\right)},
\end{gathered}
$$

and

$$
B=\frac{I_{0}(a) Y(s)-c I_{i}(a)}{s\left[K_{0}(b) I_{0}(a)-K_{0}(a) I_{0}(b)\right] Y(s)-c\left[K_{0}(b) I_{1}(a)-K_{1}(a) I_{0}(b)\right]},(40)
$$

where

$$
a=\delta_{0} s, \quad b=\delta_{0} s r_{0} / r_{i}, \quad c=\delta_{0} \mu_{i} / L d,
$$

and

$$
Y(s)=\frac{I_{1}\left(\delta_{i}^{2} s^{2}+s\right)^{\frac{1}{2}}}{\left(\delta_{i}^{2} s^{2}+s\right)^{3 / 2} I_{0}\left(\delta_{i}^{2} s^{2}+s\right)^{\frac{3}{2}}}
$$

Inversion of this solution to position and time coordinates is formidable. The transformation may be performed by the method of residues, application of which requires the poles of the equation. The poles are also equal to the negative of the time constants.

\section{Time Constants}

Since our primary concern in this investigation is the rate at which a conductor in a circuit can be brought to full current, we 
shall focus our efforts on deriving the time constant of the above solution. The poles of the solution are the roots of the denominator of $B$. To emphasize the effect of circuit size on the time constant, we rearrange the denominator of $B$ to

$$
c\left[K_{0}(b) I_{1}(a)+K_{1}(a) I_{0}(b)\right] Y(s)\left\{\frac{K_{0}(b) I_{0}(a)-K_{0}(a) I_{0}(b)}{\left[K_{0}(b) I_{1}(a)-K_{1}(a) I_{0}(b)\right] c}-\frac{1}{Y(s)}\right\}(43)
$$

The term on the left within the brackets, from here on referred to as $K$, is a function of $s$, the properties of the radial part of the circuit, and $r_{o} / r_{i}$. The term on the right within the brackets is a function of $s$ and the properties of the inner conductor. A plot of both terms within the brackets is provided in figure 2, where the ratio of $r_{o} / r_{i}$ is a parameter. $K$ appears as a straight line with a slope which becomes more negative as $r_{0} / r_{i}$ is increased. $Y(s)$ is unaltered by changes in the radius ratio. The root of equation 43 is the value of $s$ where the two terms in the brackets are equal (the point of intersection in figure 2). As the size ratio approaches infinity, one sees in figure 2 that the point of intersection approaches zero, and, thus, the time constant approaches infinity. Figure 2 further shows that $1 / Y(s)$ intersects the abscissa at the value of $s=s_{0}$ where $\delta_{i}^{2} s_{0}^{2}-s_{0}=\lambda_{0}^{2}$. $\lambda_{0}$ is the first zero of $J_{0}$, the Bessel function of the first kind, of order $0 . \lambda_{0}^{2}=5.783186 .^{3}$ $K$ intersects the abscissa at $s=0$. The time constant, $\tau^{\prime}=-1 /$ root $\equiv-1 / s_{r}$, is therefore bound between $-1 / s_{0}$ and infinity, and is a function of the size ratio $r_{0} / r_{i}$. 


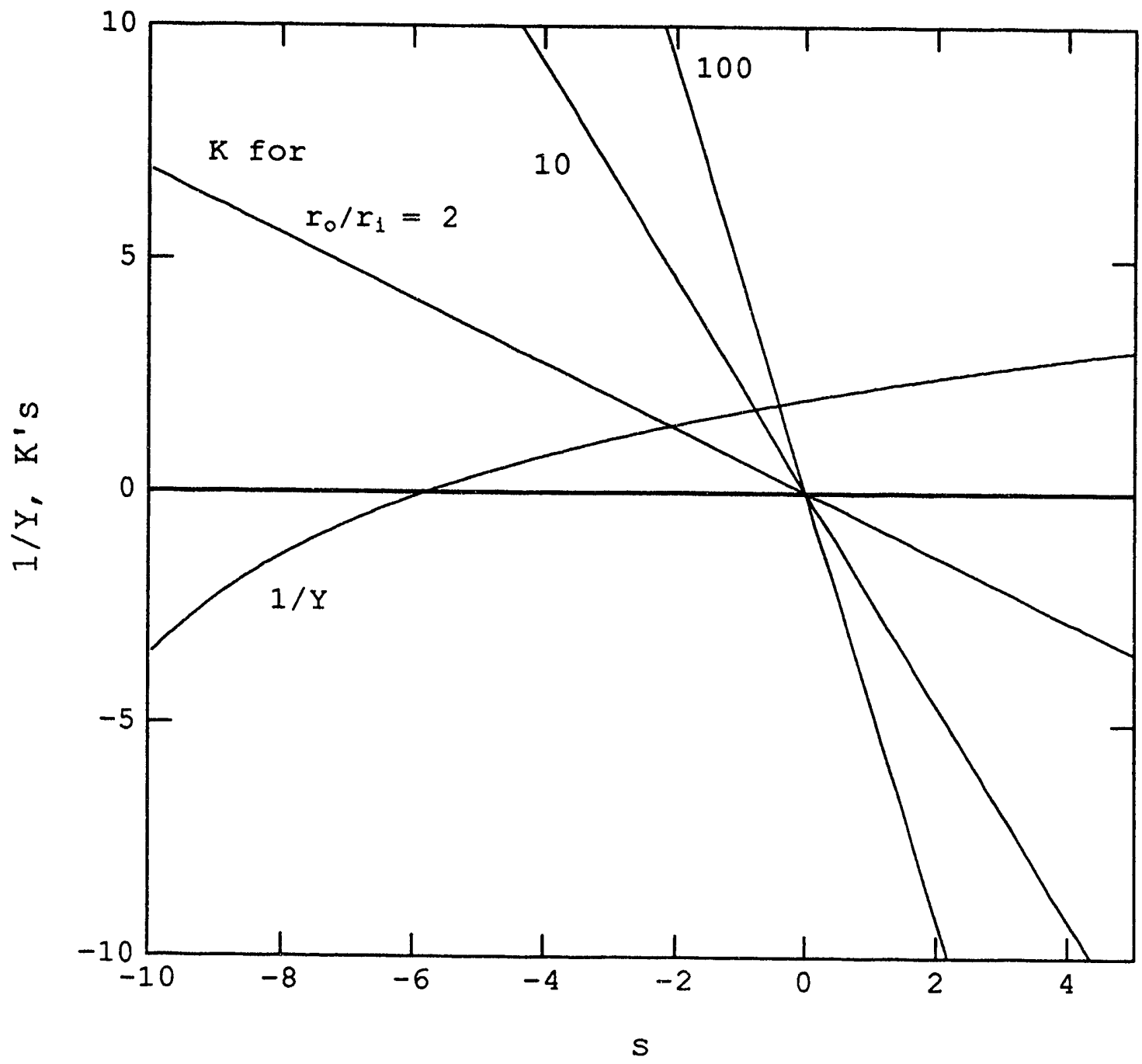

Figure 2. $\quad I / Y$ and $K$ versus $s$ for three values of $r_{0} / r_{1}$. $\delta_{1}=\delta_{0}=10^{-5}$ 
We now wish to develop an approximate analytic expression of the time constant as a function of the size ratio. The limit of $K$ as $s$ approaches zero is

$$
\lim _{s \rightarrow 0} K=-\frac{s \delta}{c} \ln \frac{r_{0}}{r_{i}} .
$$

(c is defined in equation 41.) An approximation of $1 / Y(s)$ for small $s$ is

$$
\lim _{s \rightarrow 0} \frac{1}{Y(s)} \approx 2\left(1-\frac{s}{s_{0}}\right) .
$$

Combination of the above equations leads to

$$
\frac{r^{\prime}}{r_{0}}=\frac{s_{0}}{s_{r}}=1+\frac{\left|s_{0}\right| \delta_{0}}{2 c} \ln \frac{r_{0}}{r_{i}} .
$$

This approximation of the time constant as a function of the size ratio is plotted in figure 3 along with the roots of equation 43. This figure shows that the time constant increases without bound as the ratio of the radius of the perfectly conducting outer leads to the radius of the inner conductor is increased.

\section{Case 2:}

Outer Conductors of Finite Conductivity.

The radial circuit geometry is again used here; however, this time the circuit maintains a finite conductivity. The transmissionline equation for a radial conductor with a finite conductivity is

$$
\frac{\partial^{2} \Phi}{\partial r^{2}}+\frac{1}{r} \frac{\partial \Phi}{\partial r}=L c \frac{\partial^{2} \Phi}{\partial t^{2}}+\frac{c}{\sigma_{0} l} \frac{\partial \Phi}{\partial t},
$$

as derived earlier. Substituting into the above equation the 


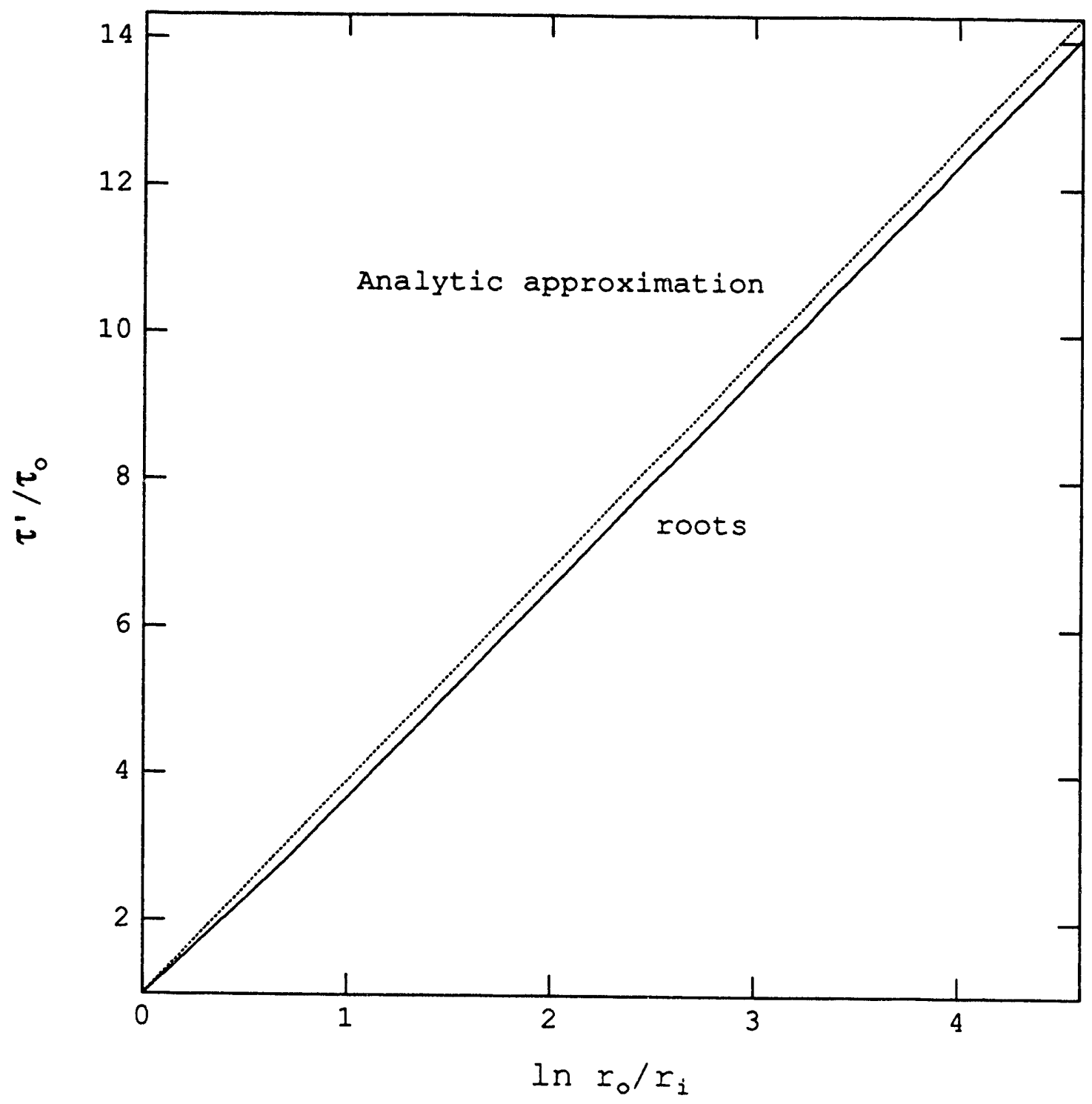

Figure 3. The time constant, and an analytic approximation, of the circuit of infinite conductivity versus the logarithm of the size ratio. 
previously defined dimensionless parameters from equation 22 gives

$$
\frac{\partial^{2} \phi}{\partial \xi^{2}}+\frac{1}{\xi} \frac{\partial \phi}{\partial \xi}=\delta_{\circ}^{2}\left(\frac{\partial^{2} \phi}{\partial \tau^{2}}+\beta \frac{\partial \phi}{\partial \tau}\right)
$$

where

$$
\beta=\frac{r_{i}^{2} \mu_{i}^{\sigma}{ }_{i}}{\sigma_{0}^{l L}} .
$$

The self-inductance of the inner conductor is proportional to its permeability, $\mu_{i}$. The resistance of the inner conductor is inversely proportional to the radius squared and the conductivity, $1 / r_{0}^{2} \sigma_{i}$. Likewise, the resistance of the outer conductor is inversely proportional to the conductivity and the thickness of the plates, $1 / l \sigma_{0}$. Thus, $\beta$ can be thought of as the ratio of the inductive time constants, $L / R$, of the inner conductor and outer radial leads.

The Laplace transform of equation 23 is

$$
\frac{\partial^{2} \bar{\phi}}{\partial \xi^{2}}+\frac{1}{\xi} \frac{\partial \bar{\phi}}{\partial \xi}=\delta_{0}^{2}\left[s^{2} \bar{\phi}-s \phi(+0)-\phi^{\prime}(+0)+\beta(s \bar{\phi}-\phi(+0))\right] .
$$

The same initial and boundary conditions apply as in case 1; however, $i_{0}$ obeys the equation from the transmission-line analysis for a conductor of finite conductivity equation 19 , which is rewritten here as

$$
-r \frac{\partial \Phi}{\partial r}=L r \frac{\partial i_{0}}{\partial \tau}+\frac{r}{\sigma I} i_{0}
$$

The dimensionless Laplace transform form of this equation is

$$
-\frac{\Phi}{r_{i}} \frac{\partial \bar{\phi}}{\partial \xi}=\left(\frac{L s}{r_{i}^{2} \mu_{i} \sigma_{i}}+\frac{1}{\sigma_{0} I}\right) \bar{i}_{0} \text {. }
$$


Combination of equations 28,36 , and 52 and some rearrangement provides the boundary condition

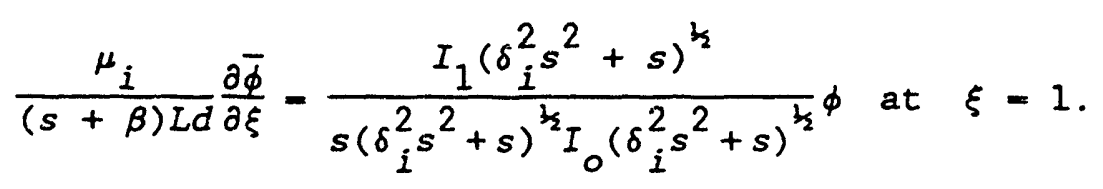

Solution

Solution of equation 48 with the derived initial and boundary conditions in equations $25,26,29$, and 53 gives the Laplace transform solution

$$
\begin{aligned}
& \bar{\phi}=A I_{0}\left[\delta_{0}\left(s^{2}+\beta s\right)^{3 / 2} \xi\right]+B K_{0}\left[\delta_{0}\left(s^{2}+\beta s\right)^{3 / 2} \xi\right] . \\
& A=\frac{\frac{1}{s}-B K_{0}(b)}{I_{0}(b)} \\
& \frac{a}{\delta_{0} c s} Y(s) I_{0}(a)-I_{1}(a) \\
& B=\frac{\delta_{0} Y(s)\left[K_{0}(b) I_{1}(a)+K_{1}(a) I_{0}(b)\right]\left\{\frac{a}{\delta \delta_{0} c s} \frac{K_{0}(b) I_{0}(a)-K_{0}(a) I_{0}(b)}{K_{1}(a)+K_{1}(a) I_{0}(b)}-\frac{1}{Y(s)}\right\}}{\text {. }} \\
& a=\delta_{0}\left(s^{2}+\beta s\right)^{\frac{1}{2}}, \quad b=\delta_{0}\left(s^{2}+\beta s\right)^{\frac{1}{2}} r_{0} / r_{i}, \quad c=\delta_{0} \frac{\mu_{i}}{L d},
\end{aligned}
$$

and $Y(s)$ is the same as in case 1 , equation 42 .

As in the first case, inversion of this solution to time coordinates is difficult; we shall again focus on obtaining the time constant of the problem.

\section{Time Constants}

We show in figure 4, as we did in figure 2, a plot of the two terms within the large brackets of equation 58 for several values of 
$\beta$ and a $r_{0} / r_{i}$ ratio of $10 / 1$. When $\beta$ is set to zero, we recover the analysis in Case 1. As $\beta$ is increased, the $K$ line from the original analysis is offset to the left by $\beta$. Again, the poles of the solution are those values of $s$ where the two curves intersect. The shift of $K$ by variations in $\beta$ precludes the time constant from approaching infinity even as the size of the circuit approaches this limit. We see that as $\beta$ is increased, the time constant, which is the inverse of the absolute value of the roots, decreases. The dimensionless time constant of a circuit of finite conductivity is constrained between the two limits $-1 / \beta$ and $-1 / s_{0}$.

Again we search for an analytic expression of the time constant. Taking the limit of the left term in the large brackets of equation 58 as $s \rightarrow 0$ gives

$$
\lim _{s \rightarrow 0} K=\frac{a^{2}}{\delta_{0} c s} \ln \frac{r_{0}}{r_{i}}=\frac{(s+\beta) \delta_{0}}{c} \ln \frac{r_{0}}{r_{i}} .
$$

The approximation of $1 / Y(s)$ for small $s$ was derived in case 1 , equation 45. Combination of the two limiting forms provides an analytic approximation of the time constant

$$
\frac{\tau^{\prime}}{\tau_{0}}=\frac{s_{0}}{s_{r}}=\frac{1-\frac{s_{0} \delta_{0}}{2 c} \ln \frac{r_{0}}{r_{i}}}{1+\frac{\beta \delta_{0}}{2 c} \ln \frac{r_{0}}{r_{i}}} .
$$

The time constants derived from the roots of equation 58 and those satisfying the above approximation are plotted versus the size of the system in figure 5 for two values of $\beta$ : one $>s_{0}$ and the other $<s_{0}$. 


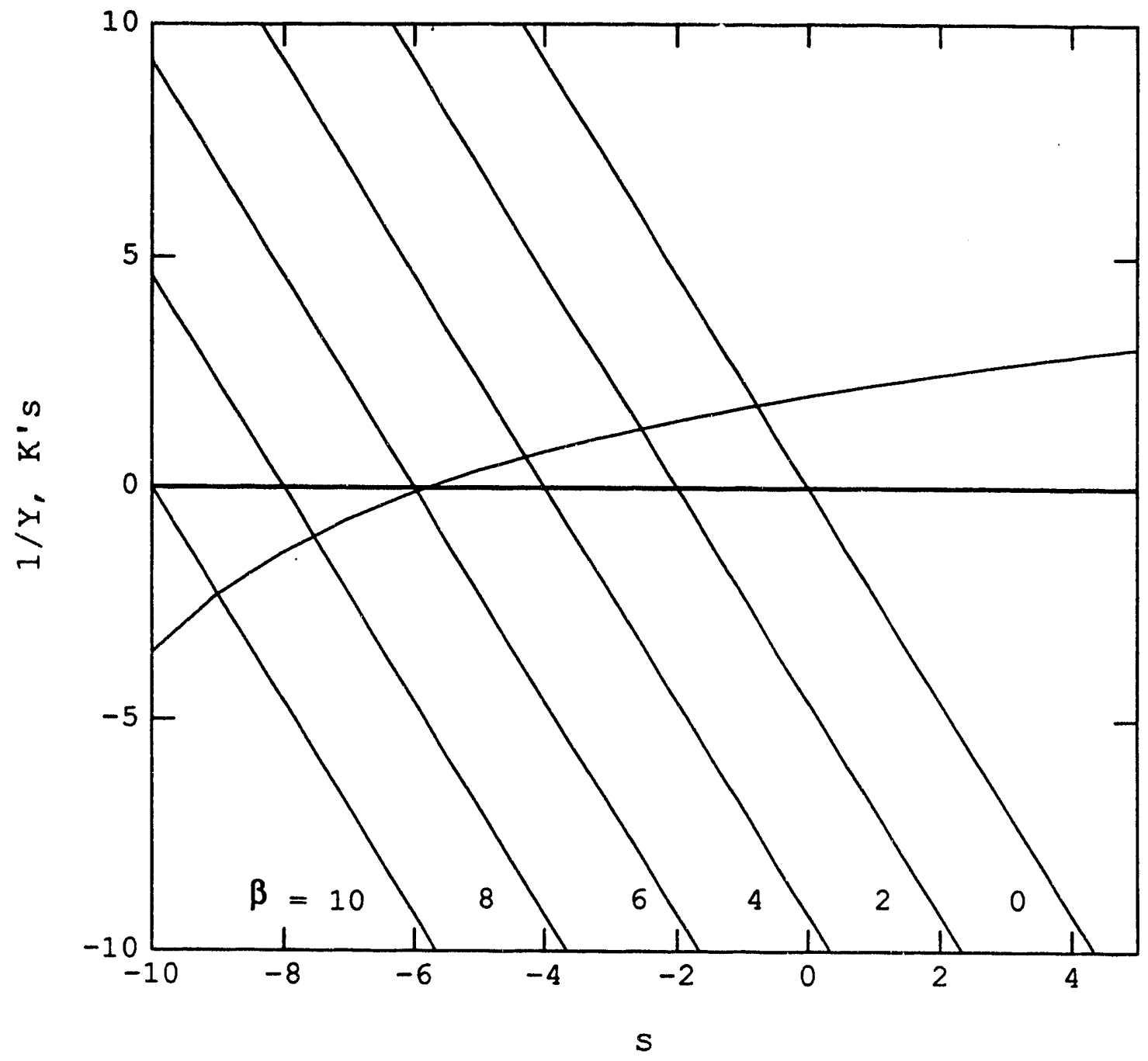

Figure 4. $\quad I / Y$ and $K^{\prime} s$ versus $s$ for different values of $\beta$. $r_{0} / r_{i}=10$. 


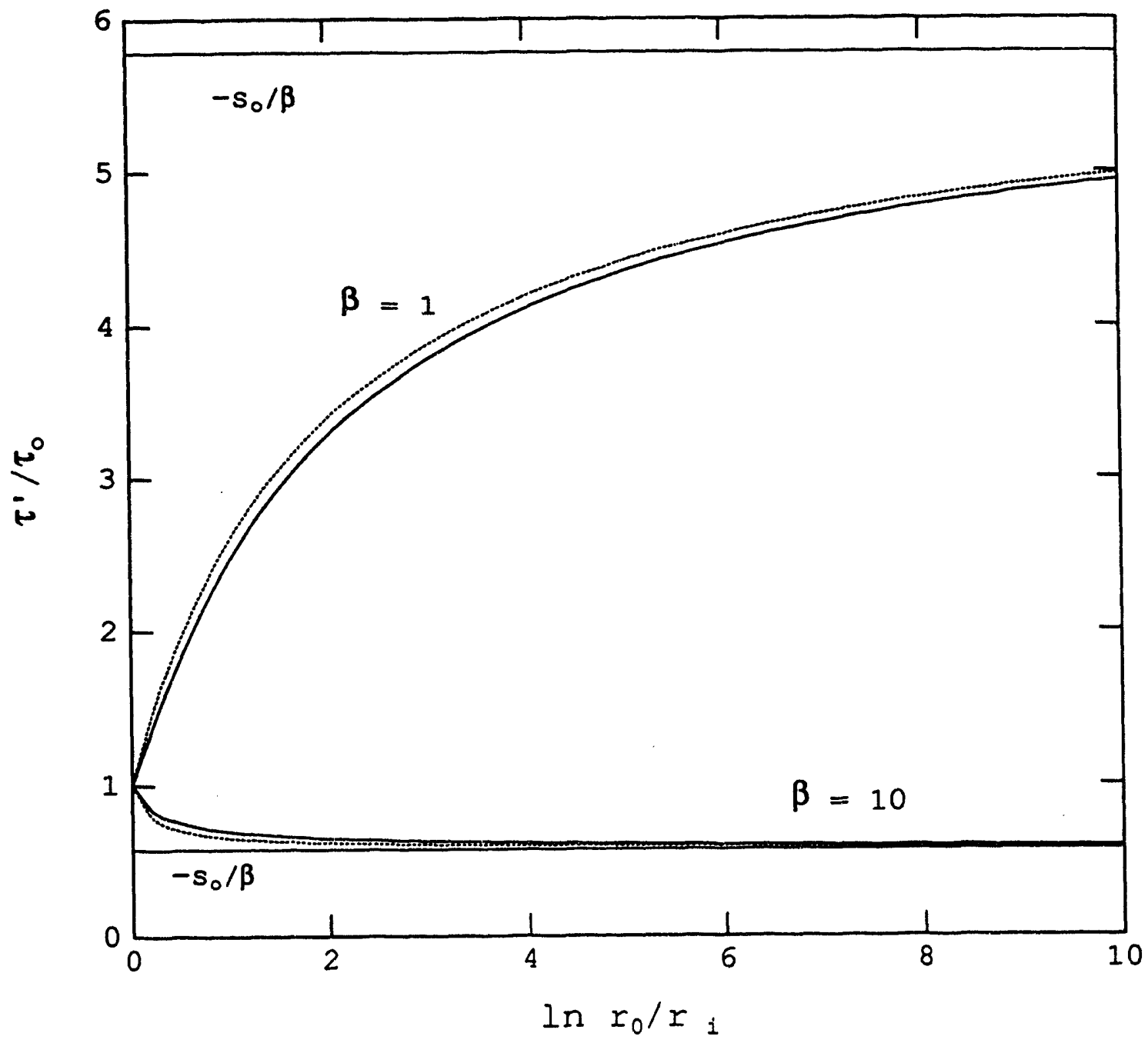

Figure 5. Time constants verses size for two values of $\beta$. The dashed curves are analytic approximations. 


\section{Discussion}

$\delta_{0}$ and $\delta_{i}$ are both typically much less than 1 and were set equal to each other and to $1 \times 10^{-5}$ in all of the figures. Only for very short times, $s$ on the order of $1 / \delta^{2}$, does the exact value of $\delta$ play a significant role. In the time frame of interest, which is the long time referred to in chapter $1, s$ is $O(1)$. The above equations can, therefore, all be simplified by setting the $\delta^{\prime} s$ to zero. This approximation has little effect on the present "long-time" results and explains why values of the $\delta^{\prime}$ 's are implicitly eliminated from the analytic approximations of the time constant derived in cases 1 and 2.

As mentioned, $\beta$ is the ratio of the inductive time constants of the inner conductor to the outer radial leads. In case 1 , where the leads are of infinite conductivity or zero resistance, the inductive time constant of the leads is infinite, and $\beta$ is zero. Setting $\beta$ to zero in case 2 reduces the solutions to those of case 1 .

For the system with infinitely conductive leads, the time constant is proportional to the logarithm of the ratio of the size of the leads to the size of the inner conductor. On the other hand, the system with leads of finite conductivity has a time constant that is bound between the time constant of the inner conductor and that of the leads. In general, the time constant of the system may either increase or decrease with the size ratio of the system, but it becomes independent of the size of the system as the size ratio approaches infinity. 
The problem we have just addressed is analogous to the problem of determining the time constant of a circuit with two inductors in series. In that situation, the inductances are added, and the resistances are added. The time constant for the circuit is then ${ }^{4}$

$$
\tau^{\prime}=\frac{L_{0}+L_{i}}{R_{0}+R_{i}}
$$

Some rearrangement gives

$$
\frac{\tau^{\prime}}{L_{i} / R_{i}}=\frac{1+L_{0} / L_{i}}{1+R_{0} / R_{i}},
$$

which is analogous to the analytic approximation provided in equation 61. This investigation demonstrates that the present method of adding inductances to determine the overall time constant is an approximation (see figures 3 and 5 ) and that the approximation is less accurate for the smaller size ratios $\left(\ln r_{0} / r_{i}=O(1)\right)$.

\section{Conclusions}

A rigorous investigation has been undertaken to determine the time constant of a radial circuit with and without resistance. The circuit with an infinite conductivity has a time constant that is proportional to the logarithm of the size of the system: as the size approaches infinity, so does the time constant. For a circuit with finite conductivity, the time constant is bound between two limits and is independent of size ratio for large systems. An analytic approximation to the time constant is provided for comparison with the rigorous evaluation and shows that the approximation, which is used in most texts of circuit analysis, is less accurate for smaller 
systems - circuits where the leads are approximately as wide as the cell of interest. This analysis is a first approximation to the time constant of a circuit containing an electrochemical power source and is an applicable design tool for rapidly discharging systems.

\section{List of Symbols}

Roman

$C$

$c$

$d$

$E$

$\bar{E}$

$i_{i}$

io

$\bar{i}_{0}$

1

L

$r$

$r_{i}$

ro

$R$

$s$

$t$ capacitance, $\mathrm{C} / \mathrm{V}$

capacitance per area, $\mathrm{C} / \mathrm{V}-\mathrm{cm}^{2}$

distance between radial conductors, $\mathrm{cm}$

electric field, $\mathrm{V} / \mathrm{cm}$

Laplace transform of $\mathrm{E}, \mathrm{V} / \mathrm{cm}$

current density in inner conductor, $\mathrm{A} / \mathrm{cm}^{2}$

current density in outer leads, $\mathrm{A} / \mathrm{cm}$

Laplace transform of $i_{0}$

thickness of radial leads, $\mathrm{cm}$

inductance, $\mathrm{V}-\mathrm{s}^{2} / \mathrm{C}-\mathrm{cm}^{2}$

radial coordinate, $\mathrm{cm}$

radius of inner conductor, $\mathrm{cm}$

radius of outer leads, $\mathrm{cm}$

resistance, $\Omega$

Laplace transform of dimensionless time, $\tau$

time, $s$ 


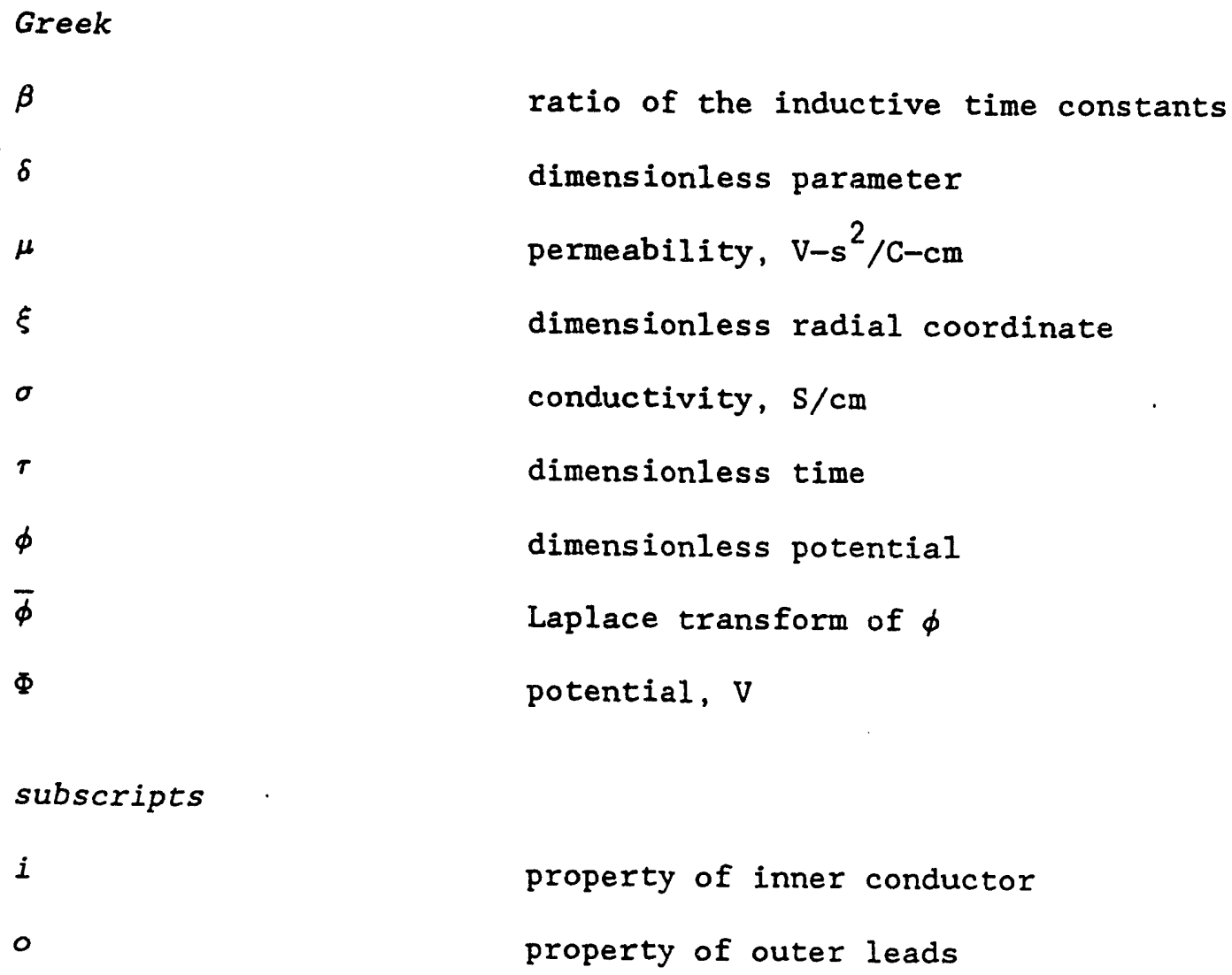

$\beta$

$\delta$

$\mu$

$\xi$

$\sigma$

$\tau$

$\phi$

$\bar{\phi}$

$\Phi$

subscripts

i

$\circ$

ratio of the inductive time constants

dimensionless parameter

permeability, $\mathrm{V}-\mathrm{s}^{2} / \mathrm{C}-\mathrm{cm}$

dimensionless radial coordinate

conductivity, $\mathrm{s} / \mathrm{cm}$

dimensionless time

dimensionless potential

Laplace transform of $\phi$

potential, V

property of inner conductor

property of outer leads

\section{References}

1. W. G. V. Rosser, "What Makes an Electric Current Flow," Am. J. Phys., 31, 884-5 (1963).

2. F. B. Hildebrand Advanced Calculus for Applications, 2nd Edition, Prentice-Hall, Inc., Englewood Cliffs, New Jersey (1976).

3. M. Abramowitz and I. A. Stegun, Handbook of Mathematical Functions, Dover Publications, Inc., New York, p.409 (1964). 
4. R. E. Thomas and A. J. Rosa, Circuits and Signals: An Introduction to Linear and Interface Circuits, John Wiley and Sons, Inc., New York (1984). 
Chapter 3

The Modeling of a Growing Oxide Film

\section{Foreword}

The full set of equations necessary for describing the growth of an oxide film is presented. The analysis includes methods of combining equations for systems with rapid kinetics and a derivation of the high-field equation. The boundary conditions on the flux equations, Poisson's equation, and the velocity of the interface are also discussed. The methodology is then applied to the iron/iron oxide system, and includes the reactions of electrons and iron interstitials at the metal/oxide and oxide/solution interfaces. Simulations, using the low-field equation, are compared to experimental results. It is found, in agreement with experiments, that passivation does not occur until $200 \mathrm{mV}$ above the potential where the formation of an oxide is thermodynamically possible. This results because the oxide formation reaction is overwhelmed by the fast kinetics of the iron dissolution to ferrous ions.

\section{Introduction}

Oxide films are present on nearly all metal surfaces subjected to oxidative environments. Those films that are compact and poor current conductors are referred to as passivating films. Experimentally it has been shown that passive films on metals, subject to an anodic current or potential, grow with a thickness that is roughly 
proportional to the logarithm of time. For over fifty years, many theories have been proposed that predict such a growth rate; and still, the most prominent theories today are but subtle improvements of some of the earliest work. (Reviews of the experiments and theories can be found in Young, ${ }^{1,2}$ Vetter, ${ }^{3}$ and Choa et al. ${ }^{4}$ )

In 1935 Verwey $^{5}$ proposed that the anodic growth of oxides is limited by the rate of transport of cationic interstitials. The driving force for migration is described by a high-field mechanism, which is exponentially dependent on the local electric field. Cabrera and Mott $^{6}$ agreed that ionic transport is dominated by cation migration by a high-field mechanism, but argued that the rate of film growth is limited by the reaction rate at the metal/oxide interface. Fehlner and Mott ${ }^{7}$ stated that ionic transport is dominated by the conduction of anions and that the limitation to growth is the reaction rate at the oxide/solution interface. Maurer ${ }^{8}$ suggested that the electricfield-dependent production of Frenkel defects - interstitials formed within the oxide by a field assisted jump of an ion from a lattice site - is the rate limiting step to forming mobile cationic species.

These theories have since been used as they stand, or combined to describe multiple phenomena, or slightly modified to fit particular system data. Vermilyea ${ }^{9}$ modified the high-field theory to describe the transport in $\mathrm{Ta}_{2} \mathrm{O}_{5}$ films to include two consecutive energy barriers to ionic transport. Burnstein and Davenport 10 adjusted the high-field model by developing an improved integration of the high-field equation. Cahan et al. ${ }^{11}$ argued that the passive 
film on iron consists of $\mathrm{Fe}^{2+}, \mathrm{Fe}^{3+}$, and $\mathrm{Fe}^{4+}$ cations, the concentrations of which vary across the film due to the presence of a strong electric field. This description is then used to characterize properties of the film and its growth. Bean et al. ${ }^{12}$ relied on a combination of cation interstitials formed through Frenkel defects and a high-field mechanism to describe the growth kinetics of tantalum oxide whereas Odynets ${ }^{13}$ claimed that the creation of defects at the interface and subsequent transport across the film are equally responsible for the limited rate of film growth. Many researchers, such as Dewald, ${ }^{14,15}$ Dignam, ${ }^{16}$ and Greyling et al., ${ }^{17}$ ascribed the differences between experimental data and the high-field transport model to neglect of the space charge in the oxide, while others 18,19 have suggested that the data can be explained by a place-exchange model.

More recent work relies on a defect model, originally developed by Frenkel. 20 This model emphasizes that current is conducted by mobile charged defects. Macdonald's point-defect models ${ }^{4,21,22}$ do not include a high-field mechanism of migration, and only the most recent paper attempts to include finite interfacial kinetics. MacDougall's point-defect models ${ }^{23,24}$ are concerned mostly with the structure of the oxide and suggest that transient changes of currents at a given potential are due to a reduction of the number of oxide imperfections. The models of Macdonald and MacDougall were recently compared by Dagan and Tomkiewicz ${ }^{25}$ for the growth of films on permalloy. They found that Macdonald's model did not fit the data as well 
as MacDougall's (although, MacDougall's paper is based solely on qualitative arguments).

In each of the above theories, only one mechanism at a time is considered such that an analytic expression for film growth is derived. These equations usually take one of the following forms: a logarithmic growth law, an inverse logarithmic growth law, or a modified inverse logarithmic growth law. Lukac et al. ${ }^{26}$ have gone on to show that these three laws can equally describe the growth kinetics and that none of the models is completely consistent with the experimentally observed temperature and potential dependences.

We believe that any or all of the above mentioned phenomena may play a significant role in oxide growth, where the predominant mechanism is a function of the system being examined and the stage to which growth has transpired. Therefore, in order to follow the progress of the growth of any oxide film through all of its stages, a model is developed that contains all of the physics. The equations of this model are then solved simultaneously with a computer. Starting with the framework of Macdonald's point defect model, we shall present a general model that includes any number of species that mav react homogeneously and heterogeneously. The model will also include effects due to variations in the adjacent solution phase and track film growth or dissolution.

In the model development we shall consider only those species that are typically present. We shall then provide the equations necessary to describe the system and touch on electric-field effects 
and thermodynamic consistency. The method used to increase the computational efficiency shall also be discussed. Upon completion of the general model development, we present results specific to the iron/iron oxide system. The first thing to be considered, however, is a description of the system.

\section{General System Description}

Figure 1 provides a schematic of the system. (This figure is similar to that introduced by Vetter, ${ }^{3}$ figure 328 .) A metal substrate interacts with a solution through an oxide. The interaction is described by reactions at both interfaces, the properties of the oxide, and the properties of the solution. The concern here is with variations occurring in a direction perpendicular to the metal interface while neglecting all parallel interactions. In other words, a one-dimensional model is developed. Furthermore, we assume that an oxide is present from the start, completely ignoring initial oxide formation (which, rigorously, occurs through nucleatinn sites and is inherently two-dimensional). A mathematically one-dimensional description of a film formed on a bare metal surface has been developed by Russell and Newman. 27

Transport in an oxide can be interpreted in terms of mobile vacancies and defects; see Wagner. ${ }^{27}$ A general model of an oxide must have the flexibility to accept various types of defects and associated reactions. Moreover, oxides may also be semiconductors; thus, electrons and holes as well as any dopant species must also be easily incorporated into the model. An account of the dominant modes 


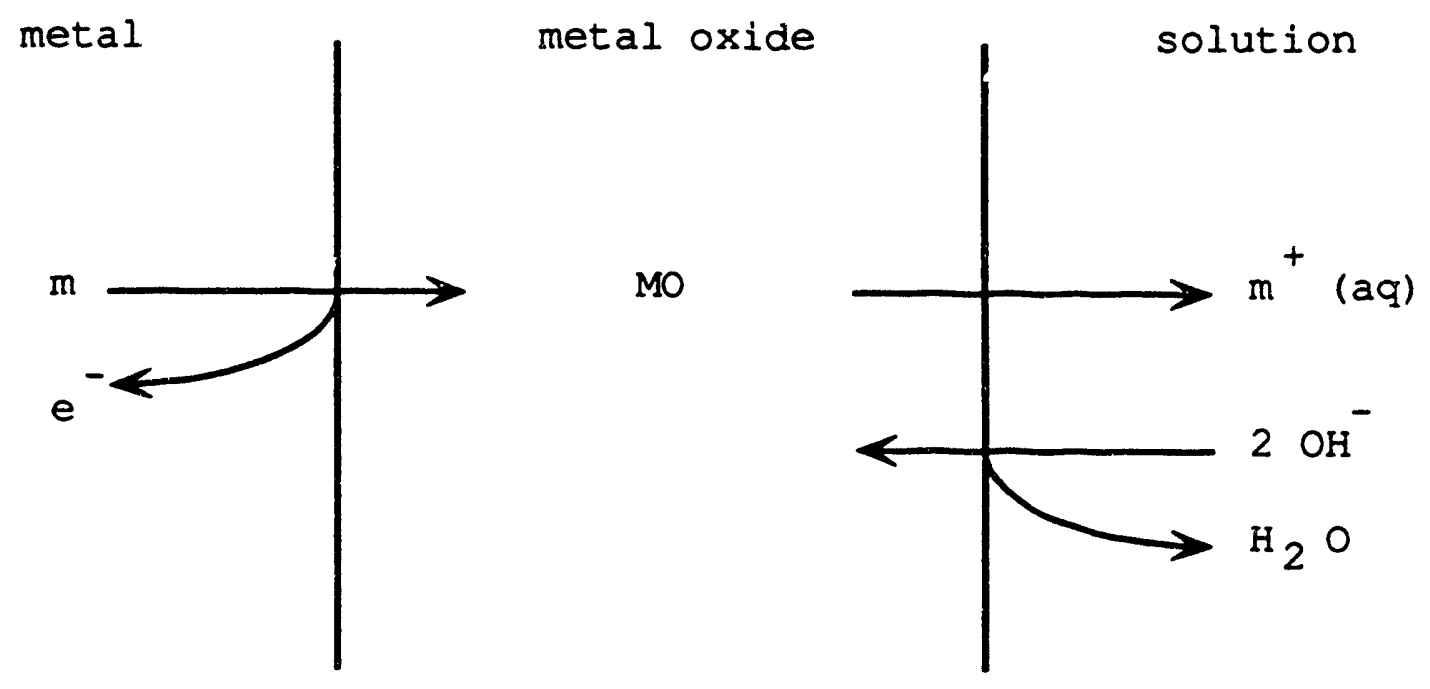

Figure 1. System. 
of conduction in oxides can be found in Shewmon ${ }^{28}$ and Sørensen. 29

To simplify the description while still providing a general framework, we shall consider only the following charge carriers: anion and cation vacancies, anion and cation interstitials, and electrons and holes. Again, we assume that the lattice itself has no charge: only the defects possess charge. More specifically, the oxide shall be viewed as an Mo background (a schematic of which appears in figure 2), where cations and anions assume their respective lattice sites, are absent from their sites, or take up interstitial locations. Kroger-Vink notation is adopted for distinguishing the species. As Choa et al. ${ }^{4}$ explain, $X_{Y}$ denotes an $X$ site occupied by a $Y$ species. Hence, $V_{M}^{X-}$ describes a metal vacancy carrying $\chi$ negative charges. Following this criterion, a cation of plus two charge in a cation site, a cation vacancy, and a cation interstitial are denoted as $\mathrm{M}_{M}, \mathrm{~V}_{M}^{2-}$, and $\mathrm{I}_{M}^{2+}$, respectively. Analogous notation is used for the anionic species. Electrons and holes appear as $e^{-}$and $h^{+}$, respectively. For our simplified description, this leads to the following types of species:

$$
e^{-} h^{+} \quad V_{M}^{2-} V_{0}^{2+} I_{M}^{2+} I_{0}^{2-} \quad M_{M} 0_{0}
$$

We shall now consider the equations necessary to describe a system consistent with this construct. 


\section{Metal Oxide}

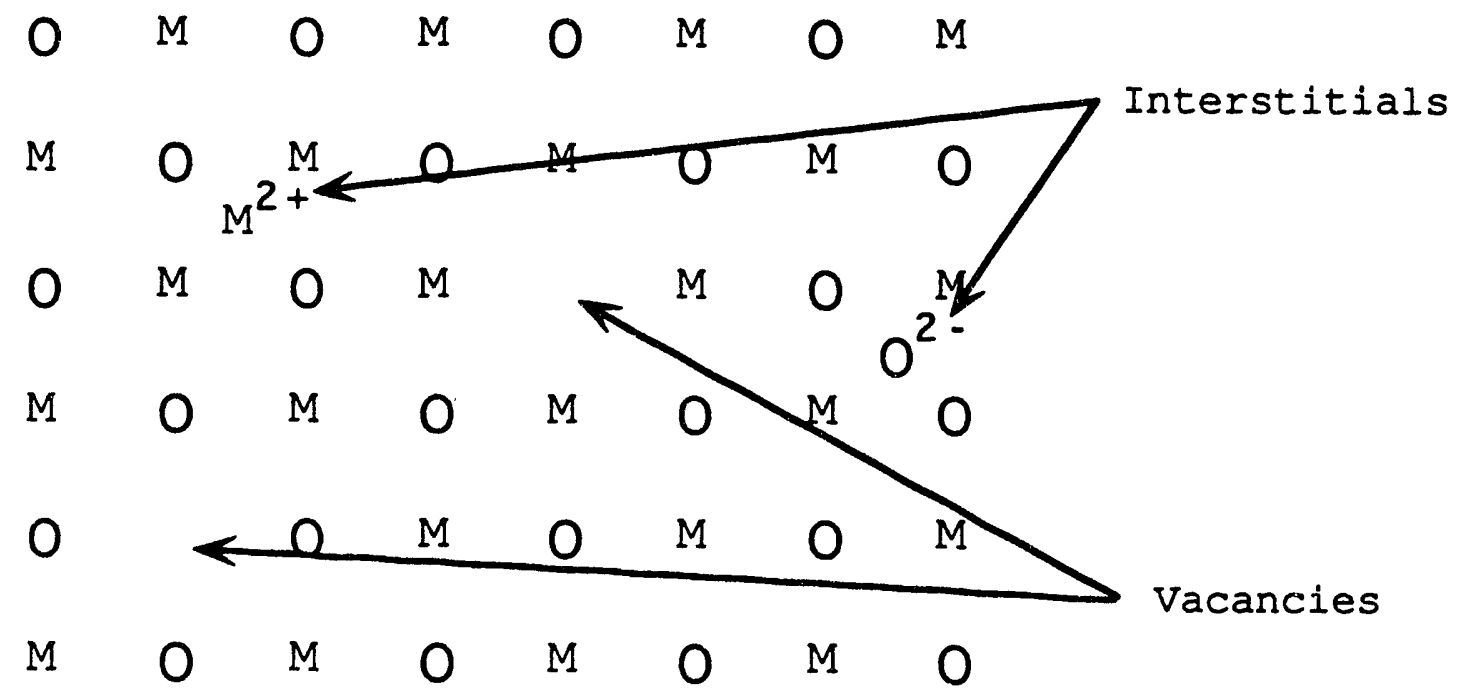

Eigure 2. Schematic of an MO oxide. 


\section{Equations}

Mole Flux Balance.

A mole flux balance for each of the species listed above may be written as

$$
\frac{\partial c_{k}}{\partial t}+\nabla \cdot \mathbf{N}_{k}=-\sum_{\ell} s_{k, \ell^{R} \ell}
$$

and read as the accumulation of a species in a differential volume plus the divergence of its flux through the faces of that volume is equal to its net generation per unit volume. The reaction rate can normally be written as the difference of forward and backward reaction terms which are a function of the activities of the species (see Newman, ${ }^{30}$ chapter 23 ). For example, a reaction that can be described by the mechanism

$$
\mathrm{V}_{0}^{2+}+\mathrm{I}_{0}^{2-} \rightarrow \mathrm{O}_{0}
$$

would typically react at a rate

$$
R_{1}=k_{1}{ }^{c} v_{0}^{2+{ }^{c}} \mathrm{I}_{0}^{2-}{ }^{-k}-1
$$

where the activity coefficients of the vacancies and interstitials and the activity of the lattice species are considered constant and are contained in the rate constants, $k_{1}$ and $k_{-1}$. Thermodynamic consistency requires $k_{1} / k_{-1}$ to be equal to the equilibrium constant, $K_{1}$, which is the ratio of the secondary-reference states of the species involved in the reaction. This particular reaction is an example of the formation of a Frenkel defect and may also be a function of the 
magnitude of the electric field. (Further discussion is deferred to the Reactions section.)

For a dilute species (which excludes $M_{M}$ and $O_{0}$ ), the flux can be written as the sum of fluxes created by separate driving forces, plus a convective term.

$$
N_{k} \equiv c_{k} v_{k} \text { - diffusior + migration + convection }
$$

The convective term is defined as the concentration of the species times a reference velocity, chosen for convenience.

The diffusion and migration terms can be interpreted as a reaction. For example, the mechanism of the transport of a cation interstitial may be depicted as an interstitial at position $\alpha$ combining with open interstitial site at position $\delta$ to form an interstitial at position $\delta$ and an interstitial site at position $\alpha$,

$$
\left(I_{M}^{2+}\right)^{\alpha}+I_{S}^{\delta} \rightleftarrows I_{S}^{\alpha}+\left(I_{M}^{2+}\right)^{\delta}
$$

Such a mechanism can be described by an Arrhenius type rate expression (Newman, 31 chapter 23). Assuming constant activity of the interstitial sites, the rate of transport appears as

$$
R_{\text {trans }}=k c_{I_{M}^{2+}}^{2+} \exp \left(\frac{z_{k} F\left(\Phi^{\alpha}-\Phi^{\delta}\right)}{2 R T}\right)-k c_{I_{M}{ }^{2+}} \exp \left(\frac{-z_{k} F\left(\Phi^{\alpha}-\Phi^{\delta}\right)}{2 R T}\right) .
$$

The difference in the electrochemical potential at $\alpha$ and $\delta$ is defined as

$$
\mu_{k}^{\alpha}-\mu_{k}^{\delta}=z_{k} F \eta_{s}=z_{k} F\left(\Phi^{\alpha}-\Phi^{\delta}-\nu^{o}\right),
$$

where $V^{0}$ is the potential difference when the net rate of transport 
equals zero. In this case, $V^{\circ}$ equals

$$
V^{0}=\frac{R T}{z_{k} F} \ln \left(\frac{c_{k}^{\delta}}{c_{k}^{\alpha}}\right) .
$$

Substitution of these definitions into the rate expression gives the high-field rate expression

$$
R_{\text {trans }}=2 k\left(c^{\alpha}{ }_{M}^{2+} c^{\delta} I_{M}^{2+}\right)^{\frac{1}{2}} \sinh \left(\frac{\mu_{M}^{\alpha} I_{M}^{2+}-\mu^{\delta}}{2 R T}\right)
$$

The difference in the electrochemical potential can be approximated as

$$
\frac{\mu_{k}^{\alpha}-\mu_{k}^{\delta}}{a}=-\nabla \mu_{k},
$$

where $a$ is some short distance. Substitution of this equation into the rate expression and assuming a small gradient in the electrochemical potential simplifies the rate expression to the linear form used in solution electrochemistry

$$
R_{\text {trans }}=-\frac{k a}{R T}\left(c_{k}^{\alpha} c_{k}^{\delta}\right)^{\frac{1}{2}} \nabla \mu_{k} .
$$

where $k a$ is thus recognized as the diffusion coefficient, $D_{k^{\prime}}$ in dilute solutions (Newman ${ }^{31}$, chapter 11). Upon defining an electrochemical activity $A_{k}$ according to

$$
\exp \left(\frac{\mu_{k}-\mu_{k}^{\theta}}{R T}\right)=c_{k} \exp \left(\frac{z_{k} F \Phi}{R T}\right)=A_{k}
$$

and substituting into the rate expression, we obtain the simplified expression 


$$
R_{\text {trans }}=\frac{2 D_{k} A_{k}}{a \exp \left(\frac{z_{k} F \Phi}{R T}\right)} \sinh \left(-\frac{a \nabla \ln A_{k}}{2}\right) .
$$

Finally, combination of the flux terms provides the high-field flux expression

$$
N_{k}=\frac{2 D_{k} A_{k}}{a \exp \left(\frac{z_{k} F \Phi}{R T}\right)} \sinh \left(-\frac{a \nabla \ln A_{k}}{2}\right)+c_{k} v_{r e f}
$$

The mole flux balance equation for the dilute species can now be expressed in terms of the dependent variables: concentration, potential, and reference velocity. Before addressing the potential, we shall first describe the method of handling homogeneous reactions that occur at such rapidity as to be considered at equilibrium.

\section{Summing Equations}

In many instances, homogeneous reactions occur at such a fast rate that it is a good approximation to assume that the reaction is at equilibrium. When this is the case, the mole flux equations are combined to eliminate the presumed equilibrium reaction from all but one of the mole balance equations. Then this one equation is replaced with the equilibrium expression,

$$
K_{\ell}=\prod c_{k}^{-s} k, \ell
$$

where $s_{k, \ell}$ are the stoichiometric coefficients of reaction $\ell$. We have designed a subroutine called "eqnprod" that sums the mole flux equations in such a way that each reaction, starting with the fastest reaction, is eliminated from every mole balance but one (if the 
specified reactions are not independent, multiple reactions may appear in the final form of the combined equations). This translates our set of $i$ mole balance equations to $i-j$ equations of the form

$$
-\sum_{1} \nu_{i, 1} R_{1}=\sum_{k} \mu_{i, k}\left(\frac{\partial c_{k}}{\partial t}+\nabla \cdot \mathrm{N}_{k}\right),
$$

and $j$ equilibrium equations. ( $\nu$ and $\mu$ are combinations of the stoichiometric coefficients derived through the elimination of the equilibrium reactions.) For those mole balance equations containing reactions that are fast but not considered at equilibrium, the equation can be divided through by the backward rate of the fast reaction. The logarithm of this equation appears as

$$
\ln k_{I_{\max }}+\sum_{k} s_{k, I_{\max }} \ln c_{k}=\ln \left[1-\frac{\sum_{k} \mu_{i, k}\left(\frac{\partial c_{k}}{\partial t}+\nabla \cdot N_{k}\right)-\sum_{1 \neq 1} \nu_{i, 1} R_{1}}{k_{b, 1_{\max }} \Pi c_{k}{ }^{q_{k, 1} I_{\max }}}\right] .
$$

The departure from equilibrium now appears as the logarithm of 1 minus a small number.

Poisson's Equation

Many oxide films, especially those that exist as compact crystal structures, possess a small number of defects, suggesting a small number of charge carriers and a large space-charge region. For a thin oxide film, the space-charge layer may extend across a significant portion of the oxide. As Dewald ${ }^{14}$ and others have shown, space charge in the oxide can have an appreciable effect on the growth rate of films. We shall therefore incorporate Poisson's equation into our 
model development

$$
\nabla^{2} \Phi=-\frac{F}{\epsilon} \sum_{k} z_{k} c_{k}
$$

This electrostatically derived formula is thermodynamically consistent for infinitely dilute solutions.

Because a double layer also exists in the solution phase near the oxide/solution interface, Poisson's equation is also invoked in this phase, replacing the electroneutrality equation,

$$
\sum_{k} z_{k} c_{k}=0 \text {, }
$$

(which is often substituted, as a first approximation, for Poisson's equation in electrolytic solutions).

Velocity

We have yet to develop an expression for the mole flux of $M_{M}$ or $O_{0}$ in terms of concentration and potential, nor have we defined the reference velocity. We shall show how these two subjects are interrelated.

The diffusion of anion and cation vacancies occurs through the mechanisms

$$
\mathrm{o}_{0}+\mathrm{v}_{0}^{2+} \rightarrow \mathrm{v}_{0}^{2+}+\mathrm{o}_{0}
$$

and

$$
\mathrm{M}_{M}+\mathrm{V}_{\mathrm{M}}^{\chi-} \rightarrow \mathrm{V}_{M}^{\chi-}+\mathrm{M}_{M} \text {, }
$$

respectively. The mechanism for anion transport can be described mathematically as, 


$$
\mathrm{N}_{0}-c_{O_{0}} \mathrm{v}_{L}=-\left(\mathrm{N}_{0}^{2+}-c_{0} v_{0}^{2+v_{L}}\right)
$$

The flux of $v_{0}^{2+}$ relative to the convective flux is equal and opposite to the flux of $o_{0}$ relative to its convective flux. An analogous relationship applies to the transport of the cation. Rearrangement of these equations leads to the definition of the lattice velocity

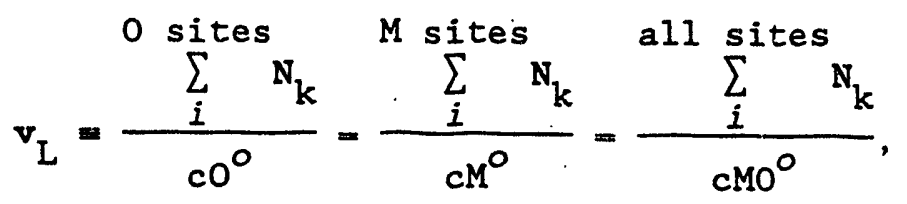

where $c_{0}^{0}, c_{M}^{0}$, and $c_{M O}^{0}$ are the concentrations of $0, M$, and $M O$ sites, respectively, and are constants. Summation of the mole flux balance equations over the species occupying $0, M$, or both sites leads to the single equation

$$
\nabla \cdot v_{L}=0
$$

This is a continuity equation consistent with the notion that the site density is constant.

In the solution, the equations that describe the velocity are the momentum-balance equations. We would prefer not to solve these equations, and instead enter velocity profiles that have been determined analytically. We shall assume that the oxide is growing on a rotating disk electrode and use the high Sc number approximation to the normal component of the velocity relative to a disk surface with no axial velocity (Newman, 31 chapter 15 ),

$$
v_{x}=-0.510235^{2} \sqrt{\Omega \nu}
$$

where $\zeta$ equals $x \sqrt{\Omega / \nu}$ and $x$ is the distance normal to the disk 
surface.

Summarizing, we have a mole balance for each minor species, Poisson's equation, and a continuity equation for the velocity. This completes the mathematical description of the transport in the bulk of the film. Completion of the problem, however, consists of a description of the boundary conditions.

\section{Boundary Conditions}

We shall now discuss the boundary conditions for a multi-phase, one-dimensional problem. For any physically realizable system there exists a certain number of degrees of freedom,

$$
f=c+2-\pi-r,
$$

at equilibrium ( $c$ is the number of species, $\pi$ is the number of phases, and $r$ is the number of independent reactions). Two of these are covered by specifying the temperature and pressure of the system. Since we typically are not at equilibrium, this equation does not necessarily apply; however it does suggest that some of the boundary conditions in one phase can not be arbitrarily set with indifference to the adjoining phases.

One method for setting boundary conditions is to mimic the experimentalist. This procedure usually leads to the correct number of constraints. For example, when a metal surface is placed in a solution with intent to form an oxide, the composition of the solution and either the potential difference across the cell or the total cell current are at the experimentalist's control. The same 
conditions are applied here. The number of degrees of freedom suggests that the boundary conditions for the oxide phase, the phase sandwiched between the metal and solution phases, are flux relationships that relate the concentration of the species in the oxide to the concentration of the species just outside the oxide. For those species that do not react at either interface nor homogeneously, there exists an additional degree of freedom. In this instance, only the initial condition is necessary for a transient problem, but for a steady-state problem one should set either the concentration of that species at one of the interfaces or an average concentration of that species. We shall proceed through the boundary conditions from one end of the system to the other for the mole flux balance, Poisson, and continuity equations.

\section{Mole Balance}

A mole flux balance equation is written for every solute species in the solution and every minor species in the oxide. This equation is second-order with respect to the concentration and therefore requires a boundary condition involving concentrations at each side of each phase. We shall start with the solution phase and go from right to left as in figure 1 with positive flux going from left to right.

Experimentally, the solution composition is typically set. We too choose to fix the concentrations in this phase. Far from the electrode we assume that the species are at equilibrium and that the fluxes of the species are constant. Combining the mole balance 
equations, as was shown above, to eliminate each reaction from all but one equation reveals the number of independent equilibrium reactions and the form of the mole balance equations. If the solvent is a dissociative species, that is, it reduces to one or more minor species, such as

$$
\mathrm{H}_{2} \mathrm{O} \rightarrow \mathrm{H}^{+}+\mathrm{OH}^{-} \text {, }
$$

then there are two ways of handling the boundary conditions: the total amount of added components is specified and the $\mathrm{pH}$ is solved for; or the $\mathrm{pH}$ is specified and the amount of added acid or base is solved for. If the $\mathrm{pH}$ is specified, the electroneutrality equation is used to determine the amount of base or acid that is required. If the amounts of each species that is added to the solution is known, then the electroneutrality equation may have to be included to determine the amount of $\mathrm{H}^{+}$or $\mathrm{OH}^{-}$present.

We provide a simple example. Ammonium hydroxide is added to $1 \mathrm{~N}$ aqueous solution of completely dissociated ferrous sulfate such that the final pH is 10 . We assume that the solution consists of the following six solute species,

$$
\mathrm{NH}_{4} \mathrm{OH}, \mathrm{NH}_{4}^{+}, \mathrm{Fe}^{2+}, \mathrm{SO}_{4}^{2-}, \mathrm{H}^{+} \text {, and } \mathrm{OH}^{-} \text {. }
$$

We require six equations. There are two equilibrium equations, one relating $\mathrm{NH}_{4} \mathrm{OH}, \mathrm{NH}_{4}^{+}$, and $\mathrm{OH}^{-}$

$$
K_{\mathrm{NH}_{4} \mathrm{OH}}=\frac{\mathrm{NH}_{4}^{+} \mathrm{OH}^{-}}{c_{\mathrm{NH}_{4}} \mathrm{OH}},
$$

and one relating $\mathrm{H}^{+}$and $\mathrm{OH}^{-}$ 


$$
K_{\mathrm{w}}=\mathrm{C}_{\mathrm{H}^{+}}{ }^{\mathrm{c}} \mathrm{OH}^{-}
$$

(the water equilibrium reaction.) Combining the mole balances to eliminate these reactions from all but two equations leads to the four total mole balances,

$$
\begin{aligned}
& c_{\mathrm{Fe}^{2+}}=c^{\circ} \mathrm{Fe}^{2+} \\
& \mathrm{C}_{\mathrm{SO}_{4}^{2-}}=c^{\circ} \mathrm{SO}_{4}^{2-} \\
& c_{\mathrm{NH}_{4} \mathrm{OH}}+c_{\mathrm{NH}_{4}^{+}}=c_{\mathrm{NH}_{4} \mathrm{OH} 2}^{\circ}+c_{\mathrm{NH}_{4}^{+}}^{\circ}
\end{aligned}
$$

and

$$
c_{\mathrm{NH}_{4} \mathrm{OH}}+c_{\mathrm{OH}^{-}}+c_{\mathrm{H}^{+}}=c_{\mathrm{NH}_{4} \mathrm{OH}^{\circ}}^{\circ}+c_{\mathrm{OH}^{-}}^{\circ}+c_{\mathrm{H}^{+}}^{\circ} \text {. }
$$

Since the original amount of ammonium hydroxide added is not known and neither is $\mathrm{C}^{\mathrm{O}} \mathrm{OH}^{-}$, we replace the last two equations with

$$
-\ln c_{\mathrm{H}^{+}}=\mathrm{pH},
$$

and the electroneutrality equation,

$$
\sum_{k} z_{k} c_{k}=0
$$

This gives us our six equations.

We now move to the oxide/solution interface, focusing on the species on the solution side. Since the concentrations of each of these species has been set "far away," and since we intend to set the total potential across the system, we use flux expressions at this interface to relate the concentrations in the adjoining phase (an 
alternative would be to use flux expressions for all but one of the species and to set the concentration of that one; this would implicitly determine the total current and preclude setting the total potential). The following general mole balance equation applies for the right side of the interface:

$$
\frac{\partial \Gamma_{k}}{\partial t}+\left(N_{k}-c_{k} v_{I}\right)=\sum_{I} s_{k, I} R_{A, I},
$$

where $v_{I}$ is the velocity of the interface. In words this equation reads: the accumulation of moles of $k$ at the the solution side of the surface plus the flux of $k$ from the surface relative to the convective flux of $k$ at the surface is equal to the rate of generation of $k$ at the surface. $\Gamma_{k}$ is the surface concentration of adsorbed species at the interface and is related to the concentration just outside the interface through the rate expression

$$
R=k_{f} c_{k} \exp \left(\frac{(1-\beta) n F}{R T}\left(\Phi^{\alpha}-\Phi^{s}\right)\right)-k_{b} \Gamma_{k} \exp \left(-\frac{\beta n F}{R T}\left(\Phi^{\alpha}-\Phi^{s}\right)\right) .
$$

If the reaction rate is fast enough to assume equilibrium, the relationship is,

$$
\Gamma_{k}=K_{s} c_{k} \exp \left(\frac{n f}{R T}\left(\Phi^{\alpha}-\Phi^{s}\right)\right)
$$

where $k_{s}=k_{f} / k_{b}$ has units of length. The surface reaction, $R_{A, I}$ is written in terms of the potent: 31 and concentrations through the Butler-Volmer equation (see Newman, ${ }^{31}$ chapter 8 , for details)

$$
R_{A, I}=k_{a} \prod_{k} c_{k}^{p_{k, 1}} \exp \left(\frac{(1-\beta) n F\left(\Phi^{0}-\Phi^{s}\right)}{R T}\right)-k_{c} \prod_{k} c_{k}^{q}{ }_{k, 1} \exp \left(-\frac{\beta n F\left(\Phi^{0}-\Phi^{s}\right)}{R T}\right) .
$$


Those interfacial reactions that are so fast as to be considered at equilibrium are replaced by an equilibrium expression, and the rest of the equations are combined to eliminate that reaction from all but one equation.

On the oxide side of the oxide/solution intertace (the left side of the oxide/solution interface according to figure 1), the following equation holds for all those species which react,

$$
\frac{\partial \Gamma_{k}}{\partial t}-\left(N_{k}-c_{k} v_{I}\right)=\sum_{I} s_{k, I} R_{A, I},
$$

where $\Gamma_{k}$ is the concentration per unit area of adsorbed species on the oxide side (left side) of the interface. For those species which do not react at either interface, heterogeneously or homogeneously, the boundary condition takes on one of the following forms: the concentration of that species at the interface is fixed; the average concentration of the species across the film is fixed,

$$
\frac{1}{x_{2}-x_{1}} \int_{x_{1}}^{x_{2}} c_{k} d x-c_{k, a v g}
$$

or the mole flux balance equation is used and one of the other two options is applied at the metal/oxide interface. If one of the first two options is used here, then the mole flux balance can be applied at the metal/oxide interface.

The boundary conditions of the oxide phase at the metal/oxice interface have implicitly been covered: the same equations developed for the right side of the oxide/solution interface are used here along with the conditions considered above for those species which do 
not react.

In the metal phase, we assume that the activities of the metal atoms and electrons are constant.

Potential

Poisson's equation is solved from one end of the system to the other. This second-order equation requires a specification of the potential at some position, the absolute value of which is arbitrary, and is chosen for convenience. For our system, the potential at the end of the solution phase farthest from the oxide is set to zero.

Gauss's law is required at the interfaces. On the solution side of the oxide/solution interface, Gauss's law takes the form

$$
\nabla \Phi^{s}-\frac{\Phi^{s}-\Phi^{0}}{\delta_{0 / s}}=-\frac{F}{\epsilon} \sum_{s k} z_{k} \Gamma_{k} .
$$

In the oxide, on the left side of the oxide/solution interface, Gauss's law appears as

$$
\frac{\Phi^{s}-\Phi^{\circ}}{\delta_{0 / s}}-\nabla \Phi^{\circ}=-\frac{F}{\epsilon_{0 k}} z_{k} \Gamma_{k} .
$$

On the right side of the metal/oxide interface, we apply Gauss's law as on the right side of the oxide/solution interface, and, finally, on the metal side we specify the potential, $v$.

At one of the interfaces we are required either to fix the potential or set the current. During a potentiostatic experiment, the potential of the metal relative to a reference electrode in the solution is fixed. We too set the potential of the metal for simu- 
lating a potentiostatic experiment. To simulate a galvanostatic experiment, either the current is set and the potential is determined from the equation

$$
i=F \sum_{k} z_{k}\left(N_{k}-c_{k} v_{I}\right)=-F \sum_{k} z_{k}\left(\frac{\partial \Gamma_{k}}{\partial t}+\sum_{I} s_{k, I} R_{A, I}\right)
$$

or the potential is set and iterated upon until the desired current density is obtained.

Velocity

The equation for the velocity of the lattice was derived by summing the mole flux equations of the species occupying lattice sites. The boundary condition is analogously developed. Depending on whether the mole flux balance equations are on the right side or the left side of the interface, summation gives

$$
v_{L}-v_{I}= \pm \frac{\sum_{k}^{\text {all sites }} \sum_{\ell} s_{k, \ell^{R} \ell}}{c_{M O}^{0}}
$$

(the plus sign applies to the right side of the interface). The right side of the above equation can also be written as

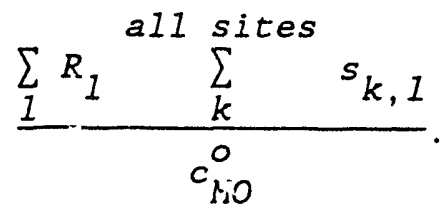

This indicates that film growth is realized only at interfaces where the sum. of the stoichiometric coefficients of species occupying lattice sites is not equal to zero. 
Velocity is a relative quantity, which allows us to set its value arbitrarily at any position. In other words, we can set the velocity of any one interface or set $v_{L}$ (since $\nabla \cdot v_{L}=0$ ) to a convenient constant. We set the velocity of the metal/oxide interface to zero.

At the metal/oxide interface, where the velocity of the interface is set to zero, the above equation yields the lattice velocity, $v_{L}$. At the oxide/solution interface it yields the velocity of the interface, since $v_{L}$ is known by integration of the continuity equation across the oxide. This $v_{I}$ can then be added directly to the velocity distribution specified in the solution phase (as shown by Acrivos $^{31}$ ).

\section{Initial Conditions}

In a transient case, an initial concentration of each species is required. One way to proceed is to set the potential of the metal to the value where the film, metal, and solution exist in mutual equilibrium. (If no such potential exists, an alternative would be to solve for a quasi-steady state at a given potential.) The steadystate concentration and potential distributions are solved for at this potential. The potential is stepped to a n?w value, and growth of the film is followed from this initial condition. To march through time, one can either use a Crank-Nicholson time stepping algorithm or assume quasi-steady state. The first method is a better approximation, especially for rapid film growth. 


\section{Reactions}

Electric Fields on Homogeneous Reactions

As we have mentioned, the gradient of the potential may enhance the forward or backward rate of certain homogeneous reactions. Why does this appear in oxides, and not in aqueous solutions? Because in oxides we are speaking of the formation or depletion of vacancies and interstitials in a lattice structure: a structure where the molecules maintain some fixed position, Thus the occupied sites and interstitials are distinguishable. A similar means of transport in a solution is described by the high-field equation.

A typical homogeneous reaction which is susceptible to influence by the electric field is the formation of Frenkel defects. This, again, is defined as interstitial species created by ions leaving the ionic sites. Oxides consist of some equilibrium concentration of interstitials and vacancies related through the relations,

$$
K_{O}=\frac{{ }^{a} o_{O}}{I_{O}^{2-{ }^{a}} v_{O}^{2+}}
$$

and

$$
K_{M}=\frac{a_{M}}{a_{M}^{2+{ }^{a}} V_{M}^{2-}} .
$$

In a nonequilibrium situation, there is reason to believe that these relationships should be independent of the potential distribution. However, if there is an alignment of the product species with the 
gradient of the potential there does exist a potential dependence. Following the procedures of chapter 23 of Newman ${ }^{31}$, we write the reaction

$$
I_{0}^{2-}+v_{0}^{2+} \rightarrow o_{0}
$$

in two ways:

$$
\text { 1. }\left(I_{0}^{2-}\right)^{\delta}+\left(V_{0}^{2+}\right)^{\alpha} \rightarrow o_{0}^{\alpha}
$$

and

$$
\text { 2. }\left(I_{0}^{2-}\right)^{\alpha}+\left(V_{0}^{2+}\right)^{\delta} \rightarrow O_{O}^{\delta} \text {, }
$$

where $\alpha$ is at one position and $\delta$ is at another position to the right. The rate of the first reaction is

$$
R_{1}=k_{f, 1} c_{I_{0}^{\delta}-{ }^{2}{ }^{\alpha} v_{O}^{2+}} \exp \left[\frac{(1-\beta) n F}{R T}\left(\Phi^{\alpha}-\Phi^{\delta}\right)\right]-k_{b, 1} c_{O}^{\alpha} \exp \left[-\frac{\beta n F}{R T}\left(\Phi^{\alpha}-\Phi^{\delta}\right)\right]
$$

and the rate of the second reaction is

$$
R_{2}=k_{f, 2}{ }^{c^{\alpha}}{ }_{I_{O}^{2-}}{ }^{c^{\delta}}{ }_{V_{O}^{2+}}^{2+} \exp \left[\frac{-(1-\beta) n F}{R T}\left(\Phi^{\alpha}-\Phi^{\delta}\right)\right]-k_{b, 1} c_{O_{O}}^{\delta} \exp \left[\frac{\beta n F}{R T}\left(\Phi^{\alpha}-\Phi^{\delta}\right)\right]
$$

The activity coefficients of the minor species and the activity of $o_{0}$ are considered constant. The summation of the two rates gives the overall rate of reaction. Substituting in the relationships

$$
\begin{gathered}
c_{k}^{\alpha}=c_{k}^{\delta}=c_{k}, \quad k_{f, 1}=k_{f, 2}=k_{f, s}, \quad k_{b, 1}=k_{b, 2}=k_{b, s}, R_{s}=z_{2}\left(R_{1}+R_{2}\right), \\
\text { and }-a \nabla \Phi=\Phi^{\alpha}-\Phi^{\delta}
\end{gathered}
$$

gives the overall rate of reaction

$$
R_{s}=k_{f, s}{ }_{I_{O}^{2-}{ }^{c} v_{O}^{2+}} \cosh \left[\frac{(1-\beta) n a F}{R T} \nabla \Phi\right]-k_{b, s} c_{O} \cosh \left[\frac{\beta n a F}{R T} \nabla \Phi\right] .
$$


At steady-state, $R_{s}=0$ and the rate equation gives

$$
\frac{\cosh \left[\frac{(1-\beta) n a F}{R T} \nabla \Phi\right]}{\cosh \left[\frac{\beta n a F}{R T} \nabla \Phi\right]}=\frac{1}{k_{s} c_{I_{O}}{ }^{c} v_{O}^{2+}} .
$$

This equation shows that the product of the concentration of species in a system at steady-state in the presence of an applied field is greater than or equal to the equilibrium constant. Further ramifications of this result are left to the reader.

\section{Thermodynamic Consistency of Heterogeneous Reactions}

Values from thermodynamic tables indicate the potential and $\mathrm{pH}$ at which a metal, in contact with an aqueous solution, will coexist simultaneously as a metal, metal oxide, and aqueous metal ions. For the system we are considering, a compact oxide covers the entire metal surface, and although the oxide is in contact with the other two phases, the other two phases are not in contact with each other. Nevertheless, if the system is at equilibrium, the same potential should be read as when there is mutual contact. This potential is, however, distributed over two interfaces, and we shall now give an example of how thermodynamic consistency is assured.

The standard-state potential versus the standard hydrogen reference electrods, $\mathrm{SHE}$, of the reactirn

$$
\mathrm{Hg}+2 \mathrm{OH}^{-} \rightleftarrows \mathrm{HgO}+\mathrm{H}_{2} \mathrm{O}+2 \mathrm{e}^{-},
$$

is 


$$
U=0.926-0.0591 \mathrm{pH}
$$

for red oxide. We shall exclude the possibility of a predominance of interstitials, vacancies, electrons, and holes in the oxide, and submit that the aqueous solution is at a certain $\mathrm{pH}$ and contains a nonobtrusive supporting electrolyte. The following reactions are considered:

at the metal/oxide interface,

$$
\text { 1.) } \mathrm{Hg} \rightleftarrows \mathrm{Hg}_{\mathrm{Hg}}+\mathrm{V}_{\mathrm{O}} \cdot 2 \mathrm{e}^{-}
$$

and

$$
\text { 2.) } \mathrm{Hg}+\mathrm{V}_{\mathrm{Hg}}^{\prime} \rightleftarrows \mathrm{Hg}_{\mathrm{Hg}}+2 \mathrm{e}^{-} \text {; }
$$

at the oxide/solution interface,

$$
\text { 3.) } 2 \mathrm{OH}^{-}+\mathrm{V}_{\mathrm{O}} \rightleftarrows \mathrm{O}_{\mathrm{O}}+\mathrm{H}_{2} \mathrm{O} \text {, }
$$

and

$$
\text { 4.) } 2 \mathrm{OH}^{-} \rightleftarrows \mathrm{O}_{\mathrm{O}}+\mathrm{V}_{\mathrm{Hg}}^{\prime}+\mathrm{H}_{2} \mathrm{O} \text {. }
$$

The individual reaction rates take the form

$$
R_{I}=k_{1} \Pi c_{k}^{P_{k, 1}} \exp \left(\frac{(1-\beta) n F\left(\Phi^{\alpha}-\Phi^{\delta}\right)}{R T}\right)-k_{-1} \Pi c_{k}^{q_{k, 1}} \exp \left(-\frac{\beta n F\left(\Phi^{\alpha}-\Phi^{\delta}\right)}{R T}\right)
$$

At equilibrium, the forward rate is equal to the backward rate; rearrangement gives

$$
\frac{n F}{R T}\left(\Phi^{\alpha}-\Phi^{\delta}\right)=\ln \frac{k-1}{k_{1}}+\sum_{k} s_{k, 1}{ }^{l n c} c_{k} .
$$

Summing reactions 1 and 3 and 2 and 4 to eliminate the vacancies leads to the overall reaction listed at the start. For a film at equilibrium, thermodynamic consistency requires 


$$
\begin{gathered}
\Phi^{m}-\Phi^{0}+\Phi^{\circ}-\Phi^{S}=U=U^{\theta}-0.0591 p H \\
=\frac{R T}{2 F} \ln \frac{k_{-1}{ }^{k}-3}{k_{1} k_{3}}-\frac{R T}{F} \ln c_{O H^{-}}=\frac{R T}{2 F} \ln \frac{k_{-2} 2^{k}-4}{k_{2} k_{4} K_{W}^{2}}-\frac{R T}{F} p H .
\end{gathered}
$$

where $U^{\theta}$ is equal to 0.926 for $\mathrm{Hg} / \mathrm{HgO}$ and $2.303 \mathrm{RT} / \mathrm{F}$ equals 0.0591 at $25^{\circ}$ C. More simply,

$$
K_{1} K_{3} K_{w}^{n}=K_{2} K_{4} K_{w}^{n}=\exp \left(\frac{n F}{R T} U^{\theta}\right) .
$$

Knowledge of the equilibrium concentrations of the defects in the oxide and of the cavity potentials across the interfaces provides a quantitative assessment of the individual $K^{\prime} \mathrm{s}$. The corresponding $K$ at the other interface is set such that the total potential drop is consistent with the overall standard-state potential drop and the local interfacial concentrations at that interface.

\section{Solving Computationally}

The equations above are linearized and differentiated with Newman's Autoband subroutine (Newman ${ }^{31}$, Appendix $C$ ). They are then solved simultaneously with Newman's Band subroutine. However, before the equations are entered, a coordinate transformation is performed.

Coordinate Transformation

To avoid extrapolation or interpolation of the concentration and potential profiles as the oxide grows or shrinks, we shall invoke a coordinate transformation. This transformation, used in each phase, is applied before assuming a quasi-steady-state condition. 


$$
x, t \rightarrow y, t
$$

where

$$
y=\frac{x-x^{\prime}}{x^{\prime \prime}-x^{\prime}}+m^{\prime}-m_{0} .
$$

$x^{\prime}$ is the position of the interface to the left of $x . x^{\prime \prime}$ is the position of the interface to the right of $x . m^{\prime}$ is the $m^{\text {th }}$ interface from the left end of the system and is the interface to the left of $x$. $m_{0}$ is the $m^{t h}$ interface from the left where $y$ is set to zero.

\section{Equations in Finite Difference}

The equations are solved using finite differences. A giant 3width banded matrix is created of $n \times n$ matrices, where $n$ is equal to the total number of species in each phase, plus the number of phases where the potential is solved for, plus the number of phases where the velocity is solved for. These equations $a_{\downarrow}$. linearized and then solved simultaneously using Newman's ${ }^{31}$ Autoband, Band, and Matinv subroutines. Iteration is required because the equations are nonlinear. The giant matrix maintains a width of three through the bulk and interfaces by following the work of Russel1. ${ }^{32}$ Here, as there, the equations are applied over a mesh spacing that is half a space wide on either side of each riode. After the coordinate transformation, the mole flux balance takes the form,

$$
\begin{gathered}
\frac{\partial c_{k}}{\partial t}-\frac{\left(y-m^{\prime}+m_{o}\right)\left(v_{I}^{\prime \prime}-v_{I^{\prime}}\right)+v_{I}^{\prime}}{I^{\prime}} \frac{\partial c_{k}}{\partial y} \\
+\frac{1}{L^{\prime}} \frac{\partial N_{k}}{\partial y}=\sum_{I} s_{k, I_{1}}
\end{gathered}
$$

which in finite difference form appears as 


$$
\begin{gathered}
\frac{\partial c_{k}(J)}{\partial t}-\frac{(J / N J(m))\left(v_{I}^{\prime \prime}-v_{I}^{\prime}\right)+v_{I}^{\prime} \frac{c_{k}(J+1)-c_{k}(J-1)}{L^{\prime}}}{2 h} \\
+\frac{1}{L^{\prime}} \frac{N_{k}\left(J+\frac{1}{2}\right)-N_{k}\left(J-\frac{1}{2}\right)}{h}=\sum_{I} s_{k, I} R_{I} .
\end{gathered}
$$

The flux expression is written in terms of concentrations, potentials, and velocities (as described earlier), and put into finite difference form. To handle the time derivative, the equation can then be put into a Crank-Nicholson form, or the time derivative can be dropped (the quasi steady-state approximation alluded to earlier).

This analysis applies through the interfaces with the half mesh points that surround each interface. Poisson's equation and the continuity equation are handled likewise.

This concludes the general formalism for describing oxide film growth. We shall now apply this methodology to the specific system of iron/iron oxide in a basic solution.

\section{The Ixon/Iron Oxide System}

The iron/iron oxide system has received a considerable amount of attention, dating back over 100 years. Vetter, Bonhoeffer, Weil, Franck, and Sato are just a few of the researchers who have performed numerous studies to try to elucidate the mechanism of its passivation. However, the system remains poorly understood. More recent research is centered on determining the structure of the oxide with spectroscopic techniques. $33,34,35,36$

Vetter ${ }^{3}$ provides an excellent review of much of the early work. In the rest of this chapter we shall outline the information provided 
in his investigation. We shall then provide a physical model that is consistent with those findings and with the defect model proposed by Wagner. ${ }^{37}$ Finally, we shall compare results from our computer simulations with the experimental data provided by Jovancicevic et al. 38 and Lukac et al. 25

Summary of Earlier Work as Provided by Vetter

The Flade potential (an experimentally measured, typically nonequilibrium, potential that marks the onset of passivation) of iron is approximately $200 \mathrm{mV}$ more positive than the potential at which the oxide $\mathrm{Fe}_{3} \mathrm{O}_{4}$ is stable in a basic solution at $\mathrm{pH}=8.4 .^{39}$ Vetter argues that the passive film is made up of a conductive layer of $\mathrm{Fe}_{3} \mathrm{O}_{4}$ that faces the metal and a nonconducting layer of $\mathrm{Fe}_{2} \mathrm{O}_{3}$ that faces the electrolyte. As the potential of the metal in contact with the solution (no oxide present) is increased, the metal preferentially dissolves as a ferrous species. The electronically conducting $\mathrm{Fe}_{3} \mathrm{O}_{4}$ oxide, the first thermodynamically stable oxide ( $-600 \mathrm{mV}$ versus the NHE), does not appear due to its rapid rate of dissolution. As the potential is further increased, the $\mathrm{Fe}_{3} \mathrm{O}_{4}$ is oxidized to $\mathrm{Fe}_{2} \mathrm{O}_{3}$. This oxide is a poor conductor and features slow dissolution kinetics. Its presence leads to passivation. The Flade potential thus falls between the potentials defined by the overall reactions 37

$$
3 \mathrm{Fe}+8 \mathrm{OH}^{-} \rightleftarrows \mathrm{Fe}_{3} \mathrm{O}_{4}+4 \mathrm{H}_{2} \mathrm{O}+8 \mathrm{e}^{-} \text {, }
$$

and 


$$
2 \mathrm{Fe}_{3} \mathrm{O}_{4}+2 \mathrm{OH}^{-} \rightleftarrows 3 \mathrm{Fe}_{2} \mathrm{O}_{3}+\mathrm{H}_{2} \mathrm{O}+2 \mathrm{e}^{-} \text {. }
$$

The second reaction is approximately $200 \mathrm{mV}$ more positive than the first.

Another thought provided on the $200 \mathrm{mV}$ discrepancy is that $\mathrm{Fe}_{3} \mathrm{O}_{4}$ and $\mathrm{Fe}_{2} \mathrm{O}_{3}$ form mixed phases with each other. (" $\gamma-\mathrm{Fe}_{2} \mathrm{O}_{3}$ lacks each ninth $\mathrm{Fe}$ ion compared to $\mathrm{Fe}_{3} \mathrm{O}_{4}$. The $\mathrm{O}^{2-}$ ions have the same crystal lattice." ${ }^{3}$ ) If $\mathrm{Fe}_{3} \mathrm{O}_{4}$ is preferentially formed near the metal and $\mathrm{Fe}_{2} \mathrm{O}_{3}$ near the electrolyte, a concentration gradient of iron ions and electrons must exist. A concentration gradient of iron ions is attended by a diffusion potential variation across the oxide. It is this potential variation that constitutes the difference between the predicted thermodynamic formation of oxide and the experimentally measured onset of passivation.

Vetter further notes that thin steddy-state films $(<100 \mathrm{~nm})$ form on metals when dissolution occurs simultaneously with film formation. His investigation revealed that the films on iron are between 2 and $10 \mathrm{~nm}$ and that $100 \pm 2 \%$ of the dissolution results as $\mathrm{Fe}^{3+}(\mathrm{aq})$.

Model

Starting with the assumption that $\mathrm{Fe}_{3} \mathrm{O}_{4}$ and $\mathrm{Fe}_{2} \mathrm{O}_{3}$ maintain the same lattice structure but differ by the presence of either cation or anion interstitials or cation or anion vacancies leads to che four possible descriptions of the oxide presented in figure 3 . $a$. and $b$. are schematics of $\mathrm{Fe}$ ions in a fixed lattice of $12 \mathrm{O}$ ions. $c$. and $\mathrm{d}$. 


$$
\mathrm{Fe}_{3} \mathrm{O}_{4}
$$

$\mathrm{Fe} \quad \mathrm{Fe} \quad \mathrm{Fe}$

a.

$\begin{array}{ccc}\mathrm{Fe} & \mathrm{Fe} & \mathrm{Fe} \\ \mathrm{Fe} & \mathrm{Fe} & \mathrm{Fe}\end{array}$
Fe
b.
$\mathrm{Fe}$

$\mathrm{Fe} \quad \mathrm{Fe}$

Ee

$\mathrm{Fe}$

$$
\mathrm{Fe}_{2} \mathrm{O}_{3}
$$

\begin{tabular}{|c|c|}
\hline $\mathrm{Fe}$ & $\mathrm{Fe}$ \\
\hline $\mathrm{Fe}$ & $V_{E e^{3-}}$ \\
\hline $\mathrm{Fe} \mathrm{h}^{\mathrm{h}^{+}}$ & $\mathrm{Fe}$ \\
\hline
\end{tabular}

Fe

$\mathrm{Ee}$

$\mathrm{Ee}$

$\mathrm{Ee}$

Fe

$\mathrm{Fe}$

Ee

$\mathrm{Fe}$

0

$e^{-0}$

0

0

0

0

c. 0

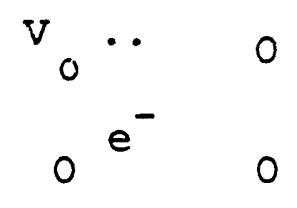

0

0

0

0

$$
0^{e^{-}} \quad 0
$$

0

0

0

0

0

0

0

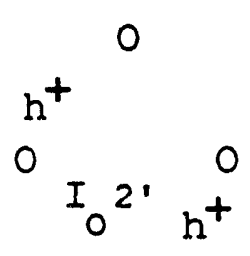

0

0

0

0

0

0 


$$
2 \mathrm{Fe}_{3} \mathrm{O}_{4}+2 \mathrm{OH}^{-} \rightleftarrows 3 \mathrm{Fe}_{2} \mathrm{O}_{3}+\mathrm{H}_{2} \mathrm{O}+2 \mathrm{e}^{-} \text {. }
$$

The second reaction is approximately $200 \mathrm{mV}$ more positive than the first.

Another thought provided on the $200 \mathrm{mV}$ discrepancy is that $\mathrm{Fe}_{3} \mathrm{O}_{4}$ and $\mathrm{Fe}_{2} \mathrm{O}_{3}$ form mixed phases with each other. (" $\gamma-\mathrm{Fe}_{2} \mathrm{O}_{3}$ lacks each ninth $\mathrm{Fe}$ ion compared to $\mathrm{Fe}_{3} \mathrm{O}_{4}$. The $\mathrm{O}^{2-}$ ions have the same crystal lattice." ${ }^{3}$ ) If $\mathrm{Fe}_{3} \mathrm{O}_{4}$ is preferentially formed near the metal and $\mathrm{Fe}_{2} \mathrm{O}_{3}$ near the electrolyte, a concentration gradient of iron ions and electrons must exist. A concentration gradient of iron ions is attended by a diffusion potential variation across the oxide. It is this potential variation that constitutes the difference between the predicted thermodynamic formation of oxide and the experimentally measured onset of passivation.

Vetter further notes that thin steady-state films $(<100 \mathrm{~nm})$ form on metals when diss. ution occurs simultaneously with film formation. His investigation revealed that the films on iron are between 2 and $10 \mathrm{~nm}$ and that $100 \pm 2$ of the dissolution results as $\mathrm{Fe}^{3+}(\mathrm{aq})$.

Model

Starting with the assumption that $\mathrm{Fe}_{3} \mathrm{O}_{4}$ and $\mathrm{Fe}_{2} \mathrm{O}_{3}$ maintain the same lattice structure but differ by the presence of either cation or anion interstitials or cation or anion vacancies leads to the four possible descriptions of the oxide presented in figure 3 . a. and $b$. are schematics of $\mathrm{Fe}$ ions in a fixed lattice of 120 ions. c. and $\mathrm{d}$. 
are schematics of $O$ ions in a fixed lattice of $6 \mathrm{Fe}$ ions. Because the $\mathrm{Fe}_{3} \mathrm{O}_{4}$ is considered the more conductive phase, a. and $d$ are eliminated as possible choices: these models lack mobile species in the $\mathrm{Fe}_{3} \mathrm{O}_{4}$ phase. X-ray diffraction experiments indicate that the oxygen lattice is fixed and that the $\mathrm{Fe}_{2} \mathrm{O}_{3}$ structure appears to be missing every ninth iron ion compared with the $\mathrm{Fe}_{3} \mathrm{O}_{4}$ structure, and work by Franck et al. 40 indicates that cation migration is the predominate mode of ionic transfer in the oxide. Thus, b. is chosen as the best description of the oxide. In words, we are assuming that the oxide takes on the structural form of $\mathrm{Fe}_{2} \mathrm{O}_{3}$ and contains mobile $\mathrm{Fe}^{3+}$ interstitials and electrons.

The following reactions are used to describe the interactions of the oxide with the adjoining metal and electrolyte phases. This is in agreement with the above model description and the information provided by Vetter.

At the metal/oxide interface:

1.) $\quad \mathrm{Fe}(\mathrm{m}) \rightleftarrows \mathrm{I}_{\mathrm{Fe}}^{3+}+3 \mathrm{e}^{-}(\mathrm{m})$.

$$
\text { 2.) } \quad \mathrm{e}^{-}(\mathrm{ox}) \rightleftarrows \mathrm{e}^{-}(\mathrm{m}) \text {; }
$$

At the oxide/solution interface:

3.)

$$
\begin{aligned}
& 2 \mathrm{I}_{\mathrm{Fe}}^{3+}+6 \mathrm{OH}^{-}(\mathrm{aq}) \rightleftarrows 2 \mathrm{Fe}_{\mathrm{Fe}}+3 \mathrm{O}_{\mathrm{O}}+3 \mathrm{H}_{2} \mathrm{O} ; \\
& \text { 4.) } \quad \mathrm{I}_{\mathrm{Fe}}^{3+}+2 \mathrm{OH}^{-} \rightleftarrows \mathrm{Fe}(\mathrm{OH})_{2}^{+}(\mathrm{aq} .) ; \\
& \text { 5.) } \quad \mathrm{I}_{\mathrm{Fe}}^{3+}+\mathrm{e}^{-}(\mathrm{ox}) \rightleftarrows \mathrm{Fe}^{2+}(\mathrm{aq} .) ;
\end{aligned}
$$


The reactions are considered to be the elementary reactions with rates of the form:

$$
\begin{aligned}
& R_{1}=k_{1} \exp \left[\frac{(1-\beta) 3 F}{R T}\left(\Phi^{m}-\Phi^{0}\right)\right]-k_{-1} c_{I_{\mathrm{Fe}}^{3+}} \exp \left[-\frac{\beta 3 F}{R T}\left(\Phi^{m}-\Phi^{0}\right)\right]
\end{aligned}
$$

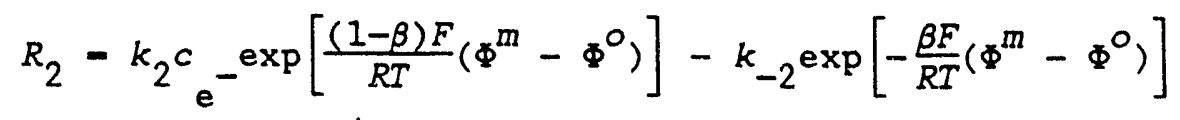

$$
\begin{aligned}
& R_{3}=k_{3} c_{\mathrm{I}_{\mathrm{Fe}}{ }^{1 / 3}{ }^{c}{ }_{\mathrm{OH}}{ }^{-}} \exp \left[\frac{(1-\beta) F}{R T}\left(\Phi^{0}-\Phi^{s}\right)\right]-k_{-3} \exp \left[-\frac{\beta F}{R T}\left(\Phi^{0}-\Phi^{s}\right)\right] \\
& R_{4}-k_{4}{ }^{c}{ }_{\mathrm{I}_{\mathrm{Fe}}{ }^{3+}{ }^{c} \mathrm{OH}^{2}}{ }^{\exp }\left[\frac{(1-\beta) 3 F}{R T}\left(\Phi^{0}-\Phi^{s}\right)\right]-k_{-4}{ }^{c} \mathrm{Fe}(\mathrm{OH})_{2}^{+} \exp \left[-\frac{\beta 3 F}{R T}\left(\Phi^{0}-\Phi^{s}\right)\right] \\
& R_{5}=k_{5}{ }_{\mathrm{I}_{\mathrm{Fe}}{ }^{3+}{ }^{c} e^{-}} \exp \left[\frac{(1-\beta) 2 F}{R T}\left(\Phi^{\circ}-\Phi^{s}\right)\right]-k_{-5}{ }^{c} \mathrm{Fe}^{2+}{ }^{\exp \left[-\frac{\beta 2 F}{R T}\left(\Phi^{\circ}-\Phi^{s}\right)\right]}
\end{aligned}
$$

Equilibrium requires:

$$
\begin{aligned}
\text { 1.) } \frac{\mathrm{I}_{\mathrm{Fe}}^{3+}}{K_{1}} & =\exp \left(\frac{3 F}{R T}\left(\Phi^{m}-\Phi^{0}\right)\right) \\
\text { 2.) } \frac{1}{K_{2^{c}} \mathrm{e}^{-}} & =\exp \left(\frac{F}{R T}\left(\Phi^{m}-\Phi^{0}\right)\right)
\end{aligned}
$$$$
\text { 3.) } \frac{1}{K_{3} c^{1 / 3}{ }_{\mathrm{I}_{\mathrm{Fe}}^{3+}{ }^{c} \mathrm{OH}^{-}}}-\exp \left(\frac{F}{R T}\left(\Phi^{0}-\Phi^{s}\right)\right)
$$$$
\text { 4.) } \frac{{ }^{c} \mathrm{Fe}(\mathrm{OH})_{2}^{+}}{K_{4}{ }^{c} \mathrm{I}_{\mathrm{Fe}}^{3+{ }^{c}{ }^{2}} \mathrm{OH}^{-}}-\exp \left(\frac{3 F}{R T}\left(\Phi^{\circ}-\Phi^{s}\right)\right)
$$

and 


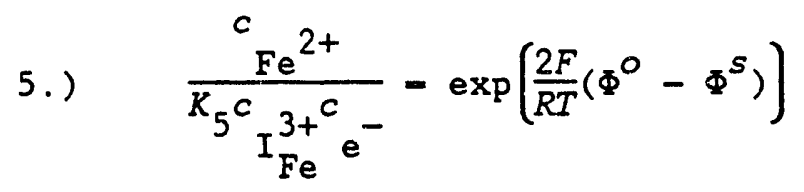

Combinations of these reactions lead to the overall reactions:

$$
\begin{gathered}
\frac{3}{4} \mathrm{Fe}(\mathrm{m})+2 \mathrm{OH}^{-} \rightarrow \frac{2}{3} \mathrm{Fe}_{\mathrm{Fe}}+\mathrm{O}_{\mathrm{O}}+\frac{1}{12} \mathrm{I}_{\mathrm{Fe}}^{3+}+\frac{1}{4} \mathrm{e}^{-}(\mathrm{ox})+\mathrm{H}_{2} \mathrm{O}+2 \mathrm{e}^{-}(\mathrm{m}) ; \\
\mathrm{Fe}(\mathrm{m})+2 \mathrm{OH}^{-} \rightarrow \mathrm{Fe}(\mathrm{OH})_{2}^{+}(\mathrm{aq})+3 \mathrm{e}^{-}(\mathrm{m}) ;
\end{gathered}
$$

and

$$
\mathrm{Fe}(\mathrm{m}) \rightarrow \mathrm{Fe}^{2+}(\mathrm{aq})+2 \mathrm{e}^{-}(\mathrm{m})
$$

It is these overall reactions for which thermodynamic constants can be found in the literature.

We shall now present the system parameters: how they were chosen or determined.

System Parameters

Table 1 provides a list of the mobile species and properties of the phases. 29,41 The electronic conductivity of $\mathrm{Fe}_{3} \mathrm{O}_{4}$ is high; therefore, an arbitrarily high diffusion coefficient for the electrons is chosen ( $100 \mathrm{~cm}^{2} / \mathrm{s}$, the same order of magnitude as found in some semiconductors). In the aqueous phase, we could not find the diffusion coefficient for the two boric species or the ferric species; however, since the current density through passive films is low $\left(\approx 5 \mu \mathrm{A} / \mathrm{cm}^{2}\right)$ and limited by transport of ions in the film, an estimate in the solution phase should be sufficient. The diffusion coefficient of interstitials for an oxide is typically small and in 


\begin{tabular}{|c|c|c|}
\hline \multicolumn{3}{|c|}{$\begin{array}{c}\text { Table } 1 \\
\text { Phase } 1: \gamma-\mathrm{Fe}_{2} \mathrm{O}_{3}\end{array}$} \\
\hline density $\left(\mathrm{g} / \mathrm{cm}^{3}\right)$ & mol. wt. (g/mol) & dielectric coeff. \\
\hline 5.24 & 55.847 & 9. \\
\hline $\begin{array}{c}\text { Mobile Species } \\
I \mathrm{Fe}^{3+}\end{array}$ & $\begin{array}{l}\text { Conc. (M) } \\
8.2\end{array}$ & $\begin{array}{c}\text { Diff. coeff. }\left(\mathrm{cm}^{2} / \mathrm{s}\right) \\
2 . \times 10^{-16}\end{array}$ \\
\hline$e^{-}$ & 24.6 & $1 . \times 10^{2}$ \\
\hline \multicolumn{3}{|c|}{ Phase 2: $\mathrm{H}_{2} \mathrm{O}$} \\
\hline $\begin{array}{c}\text { density }\left(\mathrm{g} / \mathrm{cm}^{3}\right) \\
1 .\end{array}$ & $\begin{array}{c}\text { mol. wt. (g/mol) } \\
18.0\end{array}$ & $\begin{array}{l}\text { dielectric coeff. } \\
78\end{array}$ \\
\hline Mobile Species & Conc. (M) & Diff. coeff. $\left(\mathrm{cm}^{2} / \mathrm{s}\right)$ \\
\hline $\mathrm{Na}^{+}$ & 0.20 & $1.334 \times 10^{-5}$ \\
\hline $\mathrm{Fe}^{2+}$ & $3.9 \times 10^{-6}$ & $0.72 \times 10^{-5}$ \\
\hline $\mathrm{Fe}(\mathrm{OH})_{2}^{+}$ & $1.3 \times 10^{-18}$ & $1 . \times 10^{-5}$ \\
\hline $\mathrm{B}_{4} \mathrm{O}_{7}^{2-}$ & 0.0500039 & $1 . \times 10^{-5}$ \\
\hline $\mathrm{HB}_{4} \mathrm{O}_{7}^{-}$ & 0.1 & $1 . \times 10^{-5}$ \\
\hline
\end{tabular}

this model is the mass-transfer limitation to oxide growth. Thus, the current density is limited by the rate of transport of interstitials and is directly related to the diffusion coefficient of the iron interstitials. As we shall demonstrate, this value is on the order of $10^{-16} \mathrm{~cm}^{2} / \mathrm{s}$.

Although the equilibrium constants of the overall reactions can be found in the literature, ${ }^{37}$ the values of equilibrium constants for each reaction at each interface remain t.o be determined. 
As demonstrated earlier, the equilibrium constants of the individual reactions must be consistent with the equilibrium constants of the overall reactions. The three overall reactions can be in equilibrium simultaneously if we set the $\mathrm{Fe}^{2+}$ concentration at $3.9 \times 10^{-6} \mathrm{M}$, the $\mathrm{Fe}(\mathrm{OH})_{2}^{+}$concentration to $1.3 \times 10^{-18} \mathrm{M}$, the $\mathrm{pH}$ to 8.4 and the potential versus a hydrogen reference electrode in the same solution to $-103 \mathrm{mV}(-600 \mathrm{mV}$ versus the $\mathrm{NHE})$. How does the potential vary from the metal to the reference electrode to account for this $103 \mathrm{mV}$ ? This is an important question because we are solving Poisson's equation throughout the system and Gauss's law at the interfaces simultaneously with equilibrium relationships. Thus, knowledge of the exact potential distribution across all phases is critical. This variation in potential is an equilibrium situation and is not the result of a passage of current as is the potential gradient associated with ohmic effects.

We shall now demonstrate how the potential varies from the metal to the reference electrode using our best judgement and the little data we have. We expect a potential drop between the reference electrode and the solution phase. Since we are not interested in the physics in this region, we simply require an estimate of its value to subtract from the total of $-103 \mathrm{mV}$. From potential-of-zero-charge data of the hydrogen/mercury reference electrode, it is estimated that the potential difference between the electrode and the solution is $-248.2 \mathrm{mV}$. Adding this potential to the potential difference from the metal to the reference electrode gives a potential difference 
from the metal to solution of $-351.2 \mathrm{mV}$. This potential difference can occur as a drop across the metal/oxide interface, a drop across the double layers of the oxide at both interfaces, a further drop across the oxide/solution interface, and one last drop through the solution double layer to the bulk of the solution.

The iron phase adjacent to the oxide can be thought to consist of iron ions and electrons. Since these two species are the only mobile species in both the oxide and metal phases, we shall assume that the equilibrium potential drop across this interface is zero. ${ }^{42}$ We are left with distributing the $-351.2 \mathrm{mV}$ between the oxide and the solution. We do not have information on the absorption equilibrium constants of the electrons, interstitials, sodium ions, ferrous ions, etc. at the oxide/solution interface; therefore, we shall assume that there is no specific absorption of any of the species on either side of this interface. We shall also assume that the interfacial region has a finite thickness of $0.35 \mathrm{~nm}$ and a dielectric constant that is equal to the average of the dielectric constant of the two adjacent bulk phases. With the absence of absorbed species, Gauss's law is reduced to a form that states that the gradient of the potential times the dielectric constant in the oxide just outside of the interfacial region is equal to the gradient of the potential times the dielectric constant through the interfacial region, which is also equal to the gradient of the potential times the dielectric constant in the solution double layer. Even though we do not account for specific adsorption, an equal and opposite amount of diffuse charge 
accumulates on each side of the interface to account for the potential variation and satisfy Poisson's equation.

To arrive at a first approximation of the potential and concentration distributions, which will lead to a reasonable guess of the equilibrium constants at the interface, the following iterative procedure is followed. The net accumulation of charge near an interface is related to the potential distribution near the interface by the equation 29

$$
q= \pm 2 R T \epsilon \sum_{k} c_{k, \infty}\left[\exp \frac{z_{k} F\left(\Phi_{2}-\Phi_{1}\right)}{R T}-1\right]^{3 / 2},
$$

where $\Phi_{2}$ is the potential at the interface and $\Phi_{1}$ is the potential far away in the bulk. The potential difference across the $0.35 \mathrm{~nm}$ interfacial region is related to the charge by the equation

$$
\frac{\Phi_{3}-\Phi_{2}}{0.35 \mathrm{~nm}}=\frac{\mathrm{q}}{\epsilon_{\mathrm{avg}}} .
$$

Thus, we pick a value for the charge, $q$, determine the potential drops across the oxide, interface, and solution regions, sum them to see if the total is equal to the total potential drop that is required $(-351.2 \mathrm{~V})$, and then pick another $q$ until the difference is satisfactory. Once the proper $q$ is found, we determine the concentrations at the interfaces through the equation 29

$$
c_{k}=c_{k, \infty} \exp \left[\frac{F}{R T}\left(\Phi_{2}-\Phi_{1}\right)\right] \text {. }
$$

This calculation gives us an initial guess at the potentials and concentrations at near the interfaces, and thus an initial guess in a computer program. 
The equilibrium constants of the five reactions must be consistant with the equilibrium constants of the three overall reactions. This leaves us with two degrees of freedom. The assumption that the equilibrium potential potential drop across the metal/oxide interface is zero eliminates one degree of freedom, and the assumption that the concentration of iron interstitials is equal to the bulk concentration of iron intrestitials required to make the $\mathrm{Fe}_{2} \mathrm{O}_{3}$ lattice structure into $\mathrm{Fe}_{3} \mathrm{O}_{4}$ (which is $8.2 \mathrm{M}$ ) eliminates the second degree of freedom. These values are substituted into the first equilibrium relation to obtain $k_{1}$. The concentration of electrons is related to the concentration of interstitials through electroneutrality, thus, $K_{2}$ is determinable. Substituting into the lasthree equilibrium relations the bulk concentrations and potentials provides the remaining three equilibrium constants. The equilibrium constants of reactions $1,2,3,4$, and 5 are accordingly

$$
\begin{aligned}
& K_{1}=0.040651 / \mathrm{mol}, K_{2}=8.2 \mathrm{~mol} / \mathrm{l}, K_{3}=8.57272 \times 10^{5}(1 / \mathrm{mol})^{1 / 3}, \\
& K_{4}=4.07739 \times 10^{-1} 1 / \mathrm{mol} \text {, and } K_{5}=1.44466 \times 10^{4} 1 / \mathrm{mol} . \\
& \text { We shall now present the results of our simulations and discuss } \\
& \text { the attributes and inadequacies of the model as compared to experi- } \\
& \text { mental findings. }
\end{aligned}
$$

\section{Results and Discussion}

The growth of an oxide on iron in a basic medium of boric acid and sodium borate, $\mathrm{pH}$ of 8.4 is predicted by comluter simulation. 
Generated curves are compared to experimental data. It is not our intention to present a parametric study of the diffusion coefficients and the rates of the interfacial reactions. For this reason we shall present only our best fits to the experimental data and simply describe the results of varying certain parameters. For easy comparison to experimental data, our simulations are corrected by 248.8 $\mathrm{mV}$ such that the potential of the metal is consistent with the potential one would read versus the NHE.

\section{Equilibrium}

The first result is the potential distribution for the equilibrium conditions. The system consists of a $0.1 \mathrm{~nm}$ thick oxide sandwiched between an iron surface on the left and a borate buffer solution to the right. The metal phase is set to the equilibrium potential of $-600 \mathrm{mV}$ (with the correction as discussed above) versus the potential of the solution of $-248.8 \mathrm{mV}$ at a point just outside the mass-transfer boundary layer, $12.6 \mu \mathrm{m}$ thick. The potential distribution across the oxide and solution phases is plotted against the position from the metal surface divided by the width of the particular phase, figure 4. The potential varies by approximately $47 \mathrm{mV}$ in the double layer of the oxide at the oxide solution/interface and varies by approximately $104 \mathrm{mV}$ through the double layer of the solution phase near this same interface. The rest of the $351 \mathrm{mV}$ is across the oxide/solution interface. 


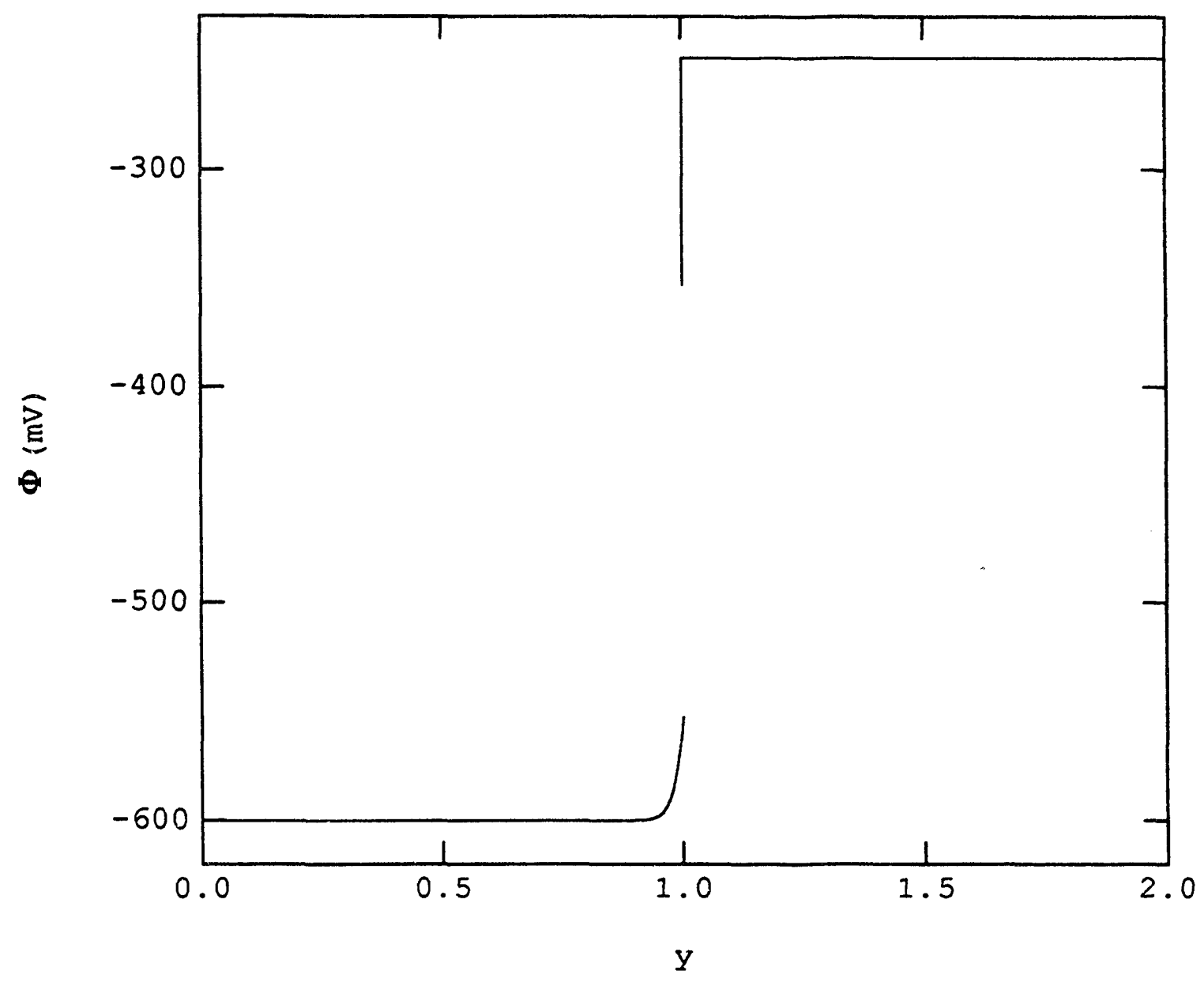

Figure 4. Potential distribution across the oxide ana solution phases at the equilibrium potential, $\mathrm{V}=-600 \mathrm{mV}$ vs. the NHE. 
The potential dis ribution in the solution appears to drop straight down at the interface. This is an illusion created by the thinness of the solution double layer $(0.61 \mathrm{~nm})$ compared to the width of the mass-transfer layer $(12.6 \mu \mathrm{m})$. The potential distribution in the oxide also reveals the thinness of the oxide double layer: about $0.015 \mathrm{~nm}$. This is considered small for most oxide semiconductors but agrees with the notion of Wagner ${ }^{35}$ for this oxide. The corresponding concentration profiles of the electrons and interstitials in the oxide are provided in figure 5. The concentration of the electrons increases by a factor of 10 near the interface, and the concentration of the interstitials drops by nearly two orders of magnitude. This is a function of the potential dependence of the concentrations as given above. Figure 6 provides the concentration and potential profiles in the solution phase versus the dimensionless coordinate $y$. This blown up view shows the curvature of the potential through the double layer near the interface and the corresponding concentration profiles. The concentrations in this region similarly obey the potential dependence given above.

the next results describe the growth of the oxide during a positive sweep of the potential.

Comparison with Experimental Data

In this part of our investigation, we introduce curves of current and length versus potential. The curves presented are those that best fit the experimental data in the literature with emphasis 


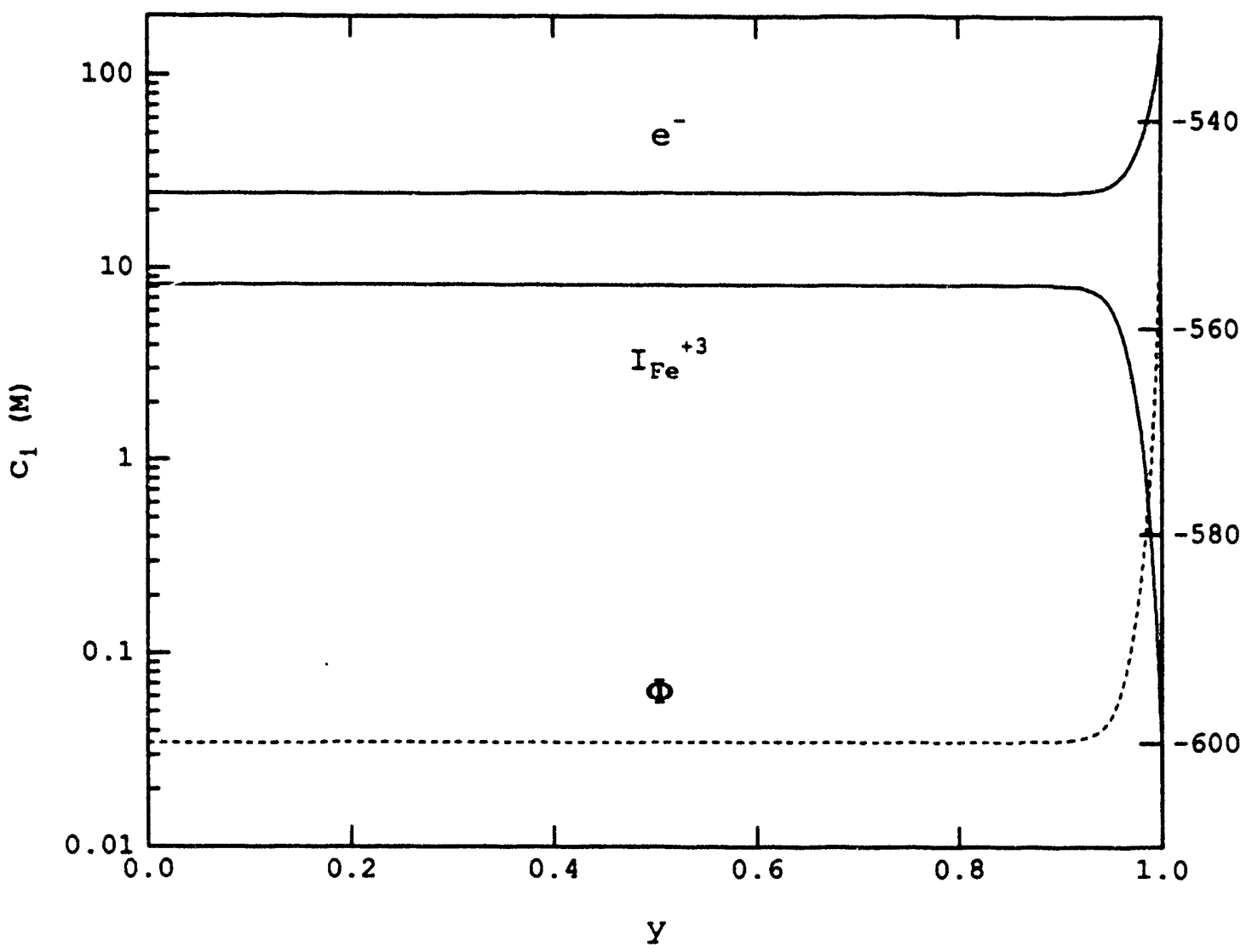

㤩
$\theta$

Figure 5. Potential and concentration distributions of interstitials and electrons versus the dimensionless coordinate, $y$, at the equilibrium potential, $v=-600 \mathrm{mV}$ vs. the NHE. 


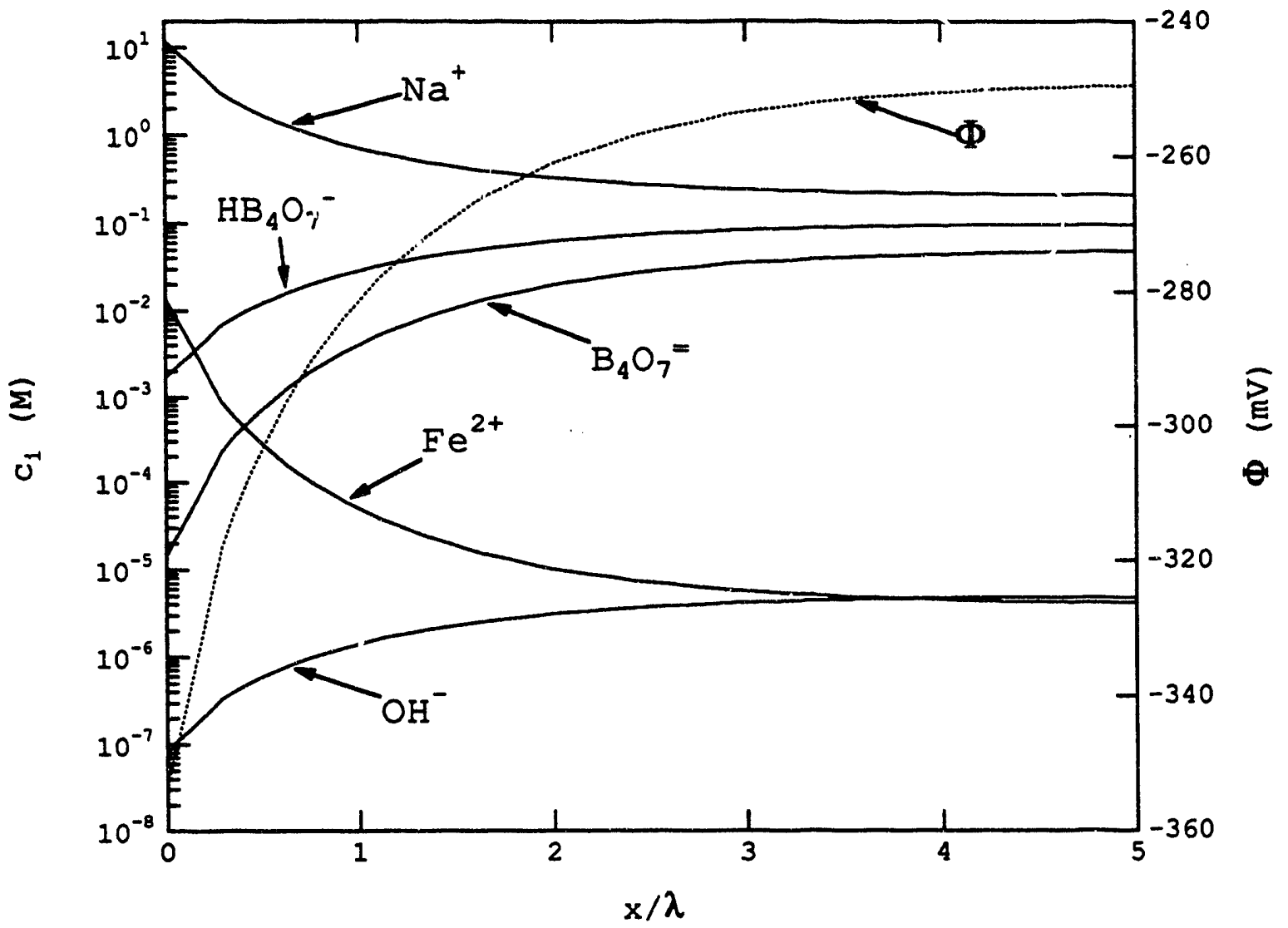

Figure 6. Concentration and potential distributions in the solution phase at the equilibrium potential versus the number of debye lengths from the oxide surface. $\mathrm{V}=-600 \mathrm{mV}$ vs. NHE . 
on emphasize important aspects of the effects of varying certain parameters. Also shown are results of simulations that include additional physics, performed to augment the comparison with the data.

Before proceeding we must state that we were unable to implement the high-field rate equation. The severe nonlinearity of this equation, combined with the high degree of coupling of Poisson's equation and the flux equations provided too difficult a challenge for Autoband $^{29}$ (the numerical differentiation subroutine we chose for solving our equations). Despite the months of effort to get around this problem, no satisfactory solution was oitained. This is clearly a disappointment, for preliminary results indicate that the field is initially growing in the high-field regime. It is felt that a proper linearization of the equations and perhaps techniques used for decoupling such problems would be effective. However, considering the amount of time that has already been soent, we decided to provide the low-field results. Moreover, it is felt that many of the trends seen in the low-field regime should hold for the high-field regime. Again, the results presented are the best fit achievable with the low-field equation.

Figure 7 contains experimental data reproduced from Jovancicevic et al. ${ }^{36}$ The current versus potential is featured for a sweep rate of $0.3 \mathrm{mV} / \mathrm{s}$. Also shown in the figure is a computer generated simulation for the same sweep rate assuming a quasi-steady state. According to Jovancicevic et al. ${ }^{36}$ an oxide of $0.03 \mathrm{~nm}$ is not seen until the potential equals $-500 \mathrm{mV}$ (this is $100 \mathrm{mV}$ above the equili- 


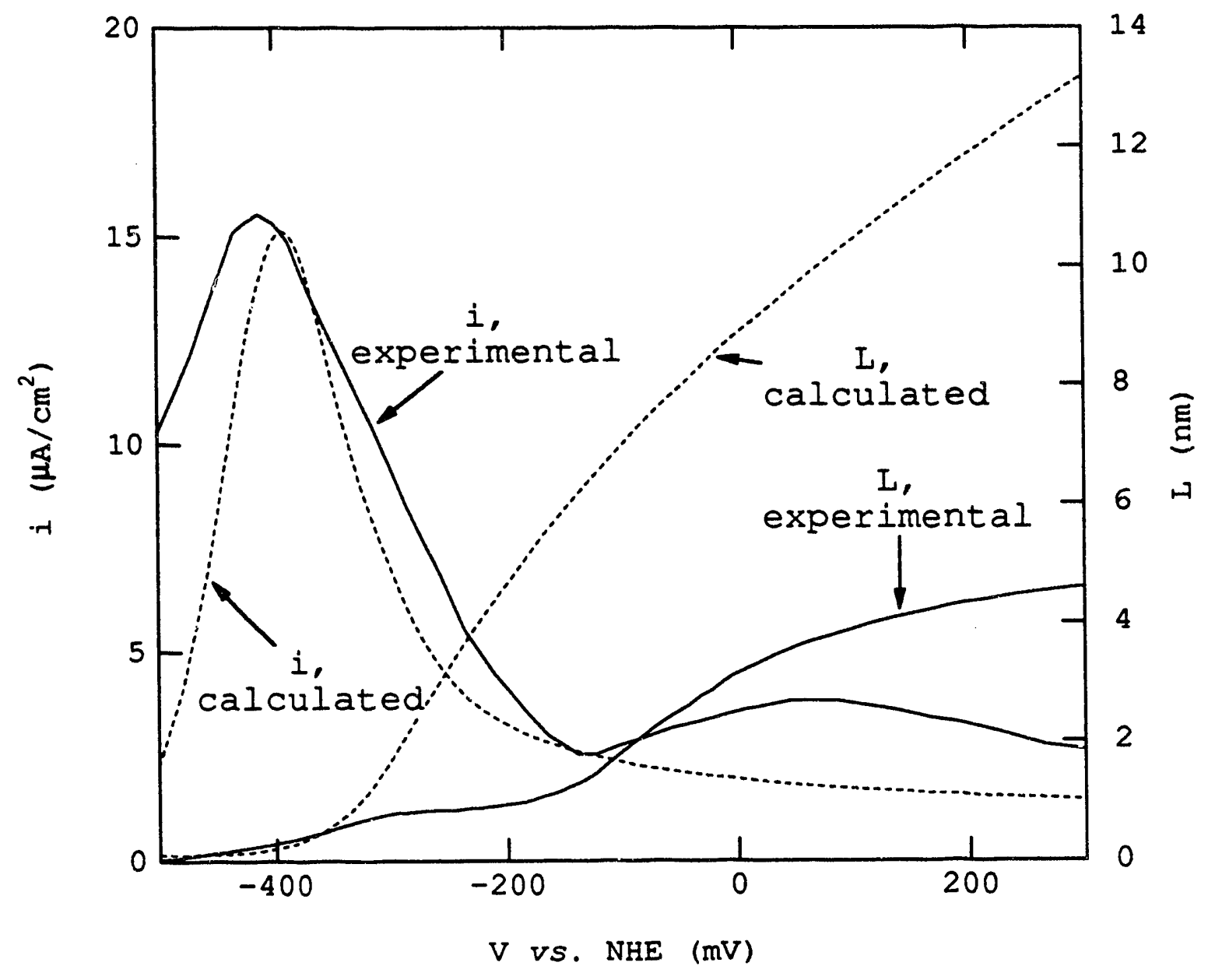

Figure 7. The solid curves are current and length versus potential data at 0.3 and $5.0 \mathrm{mV} / \mathrm{s}$, respectively, from Jovancicevic et al. ${ }^{36}$ The dashed curves are current and length versus voltage at $0.3 \mathrm{mV} / \mathrm{s}$ from simulations. 
brium potential). Since we have not included nucleation in our model, we can not simulate the results of a zero thick oxide. The simulations therefore start at $-500 \mathrm{mV}$. The $0.03 \mathrm{~nm}$ measured at this potential is thinner than an atom, and probably means that roughly 30 of the film is covered by a 1 nn thick film. Our model does not include nucleation or a means of handing the kinetics of partial coverage. The simulation will therefore provide a poor fit at the lower potentials.

To fit a curve to the data, we have at our disposal five rate constants and the diffusion coefficients of the species in the oxide. The diffusion coefficient of the interstitials is much smaller than that of any other species, and the interstitials are involved in all of the proposed reactions. It is therefore the only diffusion coefficient that we shall adjust.

The electrons move relatively easily through the oxide and, we assume, pass easily into the metal phase. Reaction 2 is therefore assumed to progress at a high enough rate to be considered at equilibrium. A variation of the rate of interstitials from the metal into the oxide should equally affect the flux of interstitials to the opposite interface. Thus, to avoid needless complications, we assume that the rate of reaction 1 progresses fast enough to be considered at equilibrium. Simulations show that the equilibrium constant for the ferric reaction, reaction 4 , is too low to have an effect, independent of its rate. For this reason the reaction is assumed to be at equilibrium (hence, maximum rate). This reduces our flexibil- 
ity to varying two rate constants, of reactions which occur at the oxide/solution interface, and the diffusion coefficient of the interstitials.

Simulations showed that as the rate constant of the ferrous reaction, reaction 5 , is increased, the initial peak in the current in figure 7 moves up and to the right with respect to potential. An increase in the oxide-forming reaction, reaction 3 , has the opposite effect. The diffusion coefficient of the interstitials moves the entire curve vertically up or down in a manner that is proportinnal to the change in the diffusion coefficient. What is going on?

Initially, as the current increases with potential, the current is limited by the rate of the ferrous reaction. The oxide-forming reaction also increases with potential, although it is not the dominant reaction, and the oxide thickens. When the current reaches a peak, the system is switching from a reaction-limited regime to a diffusion-limited regime, which defines the onset of passivation. In this regime, the current density decreases as the oxide thickens. In the diffusion-limited regime, the interstitials reach the surface at some finite rate. The interstitials are then consumed by one of the three reactions at the oxide/solution interface. As the potential is increased, the reaction that consumes the majority of interstitials will shift from a reaction of lower charge transfer to one of higher charge transfer. The charge transfer number in reaction 5 is equal to two, and the charge transfer number of reactions 3 and 4 is equal to three. Between reactions of the same order, the reaction with the 
combination of equilibrium constant and rate of reaction that allows it to appear first as the dominant means of interstitial consumption shall continue to prevail at higher potentials. For these reasons we see in figure 8 that the dominant rate of interstitial consumption switches from reaction 5 to reaction 3 . The equilibrium constant of reaction 4 is too small to allow it to compete with reaction 3 , although at the higher potentials reaction 4 carries more current than reaction 5 .

If the oxide were electrically neutral and the flux of electrons were equal to zero, the limiting current would obey the equation

$$
i_{1 \text { im }}=3 F N \underset{I_{F e}^{3+}}{ }=3 F\left(\frac{{ }^{4 D}{ }_{F e}^{3+}{ }^{c} \mathrm{I}_{\mathrm{Fe}}^{3+}}{L}\right) \text {, }
$$

where the concentration of interstitials is that at the metal/oxide interface. The diffusion-limited current of interstitials that are initially consumed primarily by the ferrous reaction is accompanied by a flux of electrons of the same direction and magnitude. The current carried by the electrons, however, is of the opposite sign and one third of the interstitial current. Thus, the limiting current appears as two thirds of that expected from the diffusive flux of interstitials alone. Figure 9 presents the current from the simulation and the theoretical limiting current and two-thirds of the theoretical limiting current when electroneutrality holds. This transition from a limiting current of interstitials accompanied by electron transport to the limiting current with no electron transport 


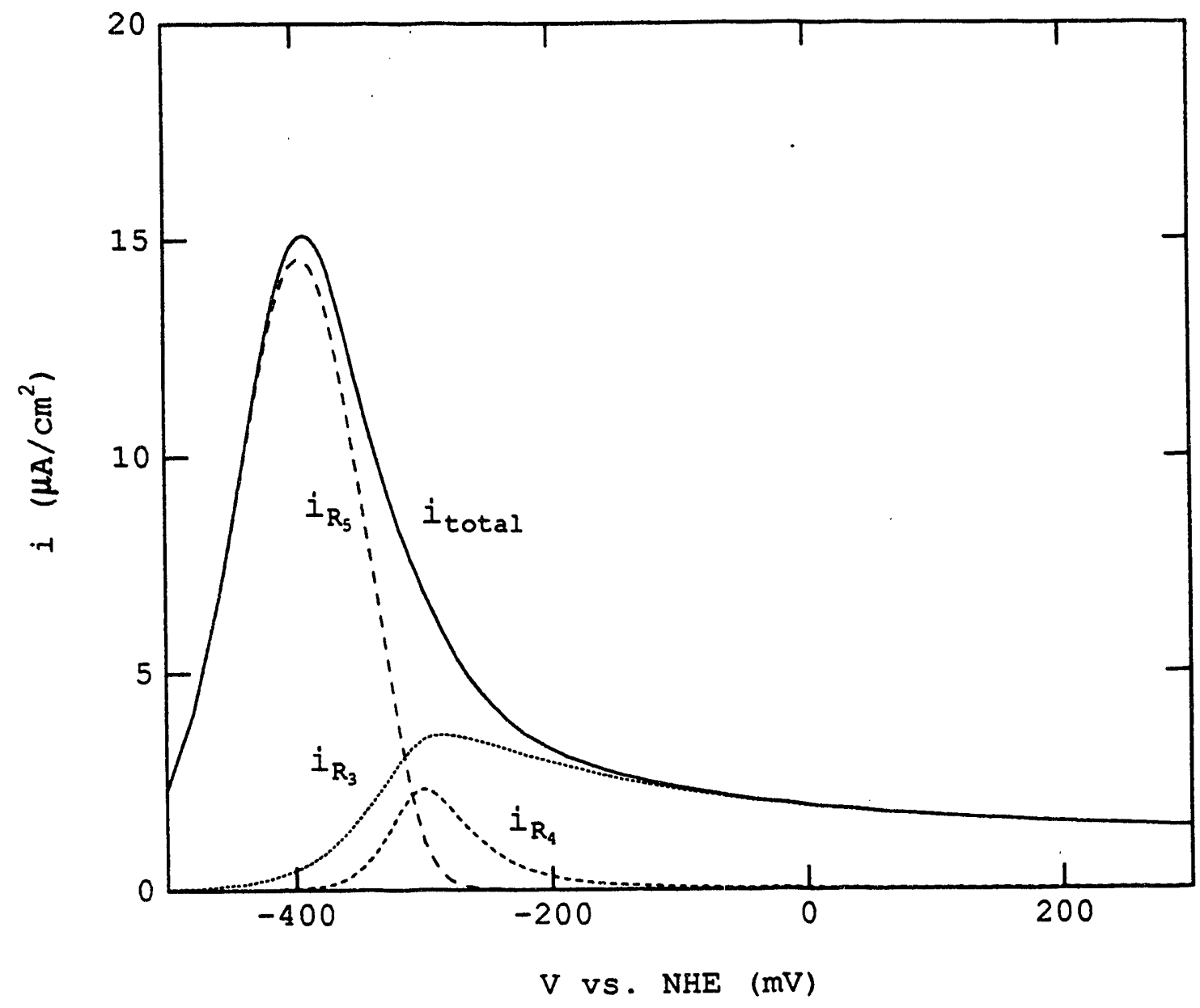

Figure 8. The currents through each reaction at the oxide/solution interface versus the potential.

Sweep rate $=0.3 \mathrm{mV} / \mathrm{s}$. 


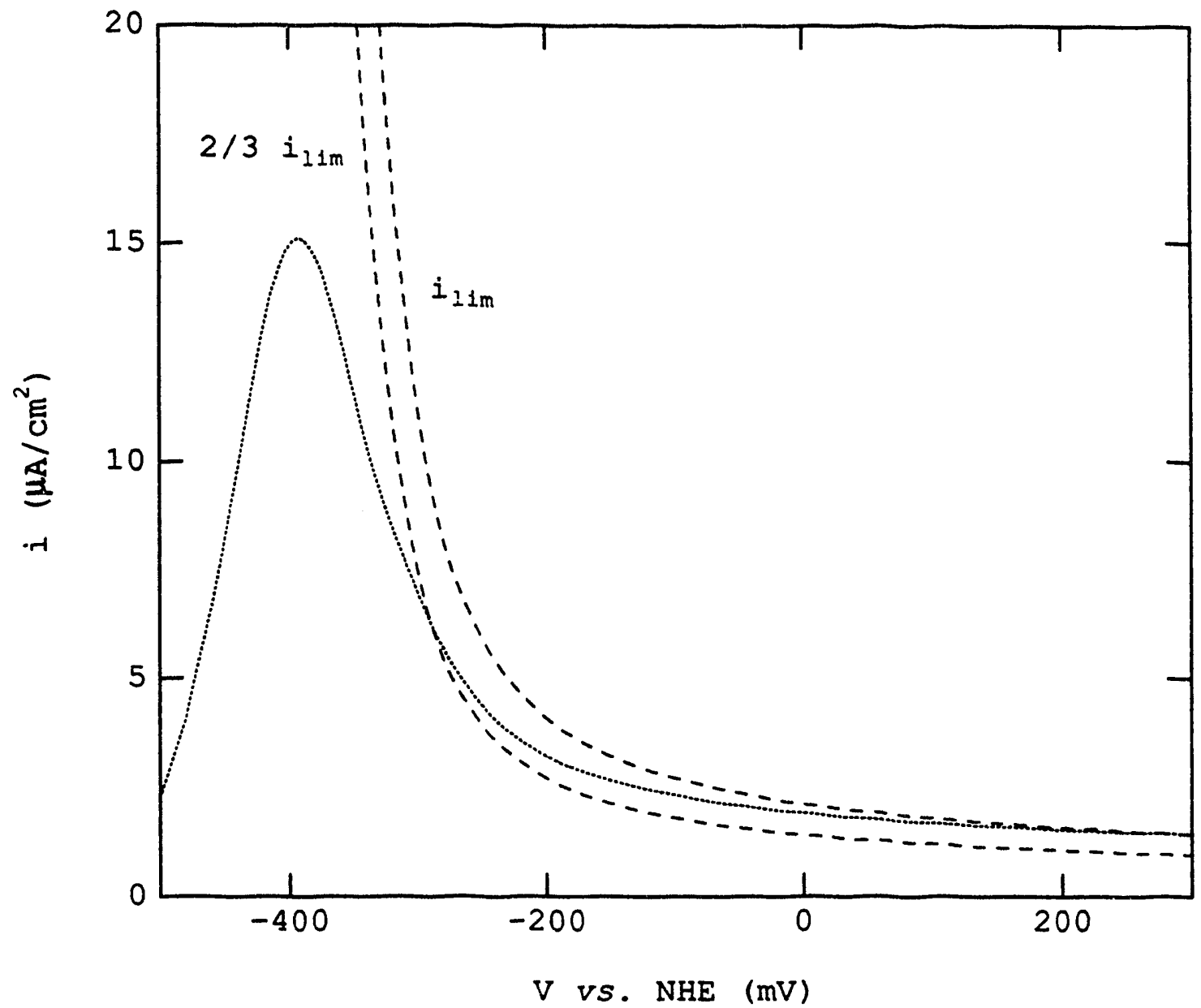

Figure 9. Current versus potential from simulation and an estimate of the limiting current and two-thirds of the limiting current. Sweep rate $=0.3 \mathrm{mV} / \mathrm{s}$. 
should follow the transition of the current from the ferrous reaction to the oxide-forming reaction. We therefore expect the two-thirds limiting current to lie on top of the simulation current at the potential where the peak starts to turn down and the full limiting current line to coincide with the simulation current at higher potentials. The lack of agreement at the low-potential end is a result of the nonelectroneutrality. This provides further justification for not making this assumption and using Poisson's equation instead.

In the end, the values that provide the best fit to the data are $k_{-3}=1 \times 10^{-13} \mathrm{~mol} / \mathrm{cm}^{2}-\mathrm{s}, k_{5}=1 \times 10^{-i 1} \mathrm{~cm} / \mathrm{s}$, and $D_{\mathrm{Fe}}^{3+}=2 \times 10^{-16} \mathrm{~cm}^{2} / \mathrm{s}$.

Further inspection of the data provided by Jovancicevic et al. reveals a second peak in the current at a potential of around $100 \mathrm{mV}$. Our first inclination was that this is the result of another reaction, perhaps the ferric reaction, reaction 4, becoming the dominant means of interstitial utilization. The simulations above were obtained assuming that reaction 4 is at equilibrium. It is therefore impossible to increase the rate of this reaction. But perhaps the equilibrium constant reported for this reaction was incorrect, and maybe there is room for adjusting its value. However, this reaction has the same reaction order as that of the oxide-film reaction. Therefore, the equilibrium constant must be increased to the point where the rate of reaction 4 overcomes the rate of reaction 3 , the oxide-forming reaction. If this is done, the oxide-film reaction is diminished, and the current continues to increase with potential, 
switching from the ferrous reaction to the ferric reaction. Eventually, enough oxide would be formed at the slow reaction rate to 1 imit the rate of transport of interstitials, and the current would slowly decrease. The final result would still be one large peak in the current.

Underpotential Deposition

The thickness of the oxide measured versus the potential during a sweep rate of $5 \mathrm{mV} / \mathrm{s}$ is provided in figure 7 . A plateau in the oxide thickness at around $7.5 \mathrm{~nm}$ appears between -375 and $-175 \mathrm{mV}$. This leads us to believe that there may be underpotential deposition. Underpotential deposition (UPD) is the deposition of any solid on a substrate made of a different material than the deposit where the substrate is thermodynamically more stable for deposition than the deposit. Deposition of this type is analogous to the BET isotherm where the surface coverage of gas molecules is a function of the pressure of the surrounding gas. We would like to take advantage of this similarity and use the equations previously derived in the literature for the BET isotherm. A driving force for deposition that is a function of the thickness of the oxide is subsequently developed.

The BET isotherm ${ }^{43}$ is characterized by the equation

$$
v=\frac{v_{m} c P}{\left(P_{0}-P\right)\left[1+\frac{(c-1) P}{P_{0}}\right]},
$$


where $v$ is the volume of the adsorbed layer, $v_{m}$ is the volume of $a$ monolayer of the absorbed layer, $c$ is a constant related to the heat of adsorption, and $P_{0}$ is the saturation pressure. In relation to our analysis, the volume of the absorbed layer is analogous to the thickness of the oxide, $L$, and the pressure is analogous to the applied potential, $U$ :

$$
v \longleftrightarrow L \text { and } \ln \frac{P}{P_{0}} \longleftrightarrow \frac{n F U}{R T} .
$$

We require an equation that gives the potential as a function of the length. Rearrangement of the above equation and substitution of $L$ and $U$ give

$$
\frac{n F U}{R T}=\ln \frac{2 P_{0}}{\left\{\left[c\left[\frac{L m}{L}-1\right]+2\right]^{2}+4(c-1)\right\}^{\frac{1}{2}}+c\left[\frac{L m}{L}-1\right]+2} .
$$

$c$ is related to the width of the potential plateau, $U_{0}$, through the equation

$$
c=\exp \left(\frac{F U_{0}}{R T}\right) .
$$

From the data of Jovancicevic et al., ${ }^{36}$ we estimate that $U_{0}$ is approximately $200 \mathrm{mV}$.

The BET isotherm relates the volume of coverage to the gas pressure. The surface coverage is not strictly associated with either interface. In this analysis, a relationship between the potential of oxide deposition and the amount of coverage is also not strictly associated with either interface. However, we felt that the best way 
to incorporate the UPD was to attribute it to the oxide-forming reaction, reaction 3 , at the oxide/solution interface. Thus, the $U$ defined above was subtracted from the potential difference of the oxide/solution interface in both the forward and reverse reaction rates of reaction 3 ,

$$
R_{3}=k_{3} c_{\mathrm{I}_{\mathrm{Fe}}{ }^{1 / 3}{ }^{c}{ }_{\mathrm{OH}}}-\exp \left[\frac{(1-\beta) F}{R T}\left(\Phi^{0}-\Phi^{s}-U\right)\right]-k_{-3} \exp \left[-\frac{\beta F}{R T}\left(\Phi^{0}-\Phi^{s}-U\right)\right]
$$

The result of the above adjustment to reaction rate 3 on the current and the length of the oxide when the potential is swept at a rate of $0.3 \mathrm{mV} / \mathrm{s}$ is provided in figure 10 . One sees a peak in the current at the same potential where the length of the film briefly levels off. The peak in the current appears because the oxide stops growing, and thus, the diffusion-limited current stops decreasing. The current tends to level off. A few millivolts further to the right in figure 11 the oxide film reaction begin to increase again, while the current drops off again.

The reason we included UPD in our analysis was not only to make our length versus potential curve agree with the experimental data but also to get the second peak in the current to agree as well. The peak we get is nowhere near the potential of the second peak in the experimental data. In our simulations, the leveling off of the oxide is going to appear at the same potential where the peak in the current appears. In the experiments, these two phenomena are separated by $200 \mathrm{mV}$. Figure 10 demonstrates that if we try to match the experimentally measured leveling off of the film thickness with 


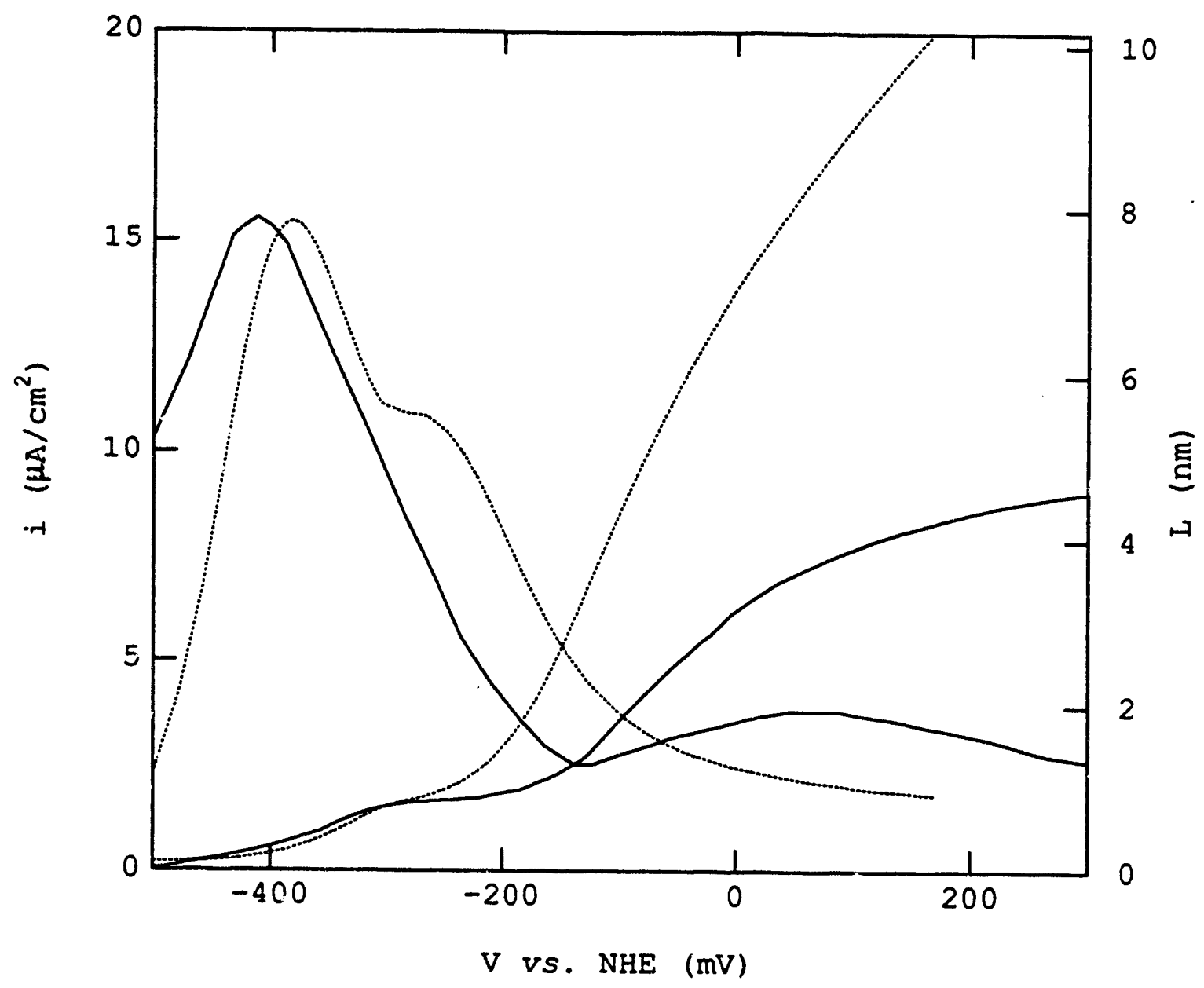

Figure 10. Solid curves are current and length versus potential data at 0.3 and $5 \mathrm{mV} / \mathrm{s}$, respectively, from Jovancicevic et al. ${ }^{36}$ The dashed curves are current and length versus voltage at $0.3 \mathrm{mV} / \mathrm{s}$ from simulations that include UPD. 


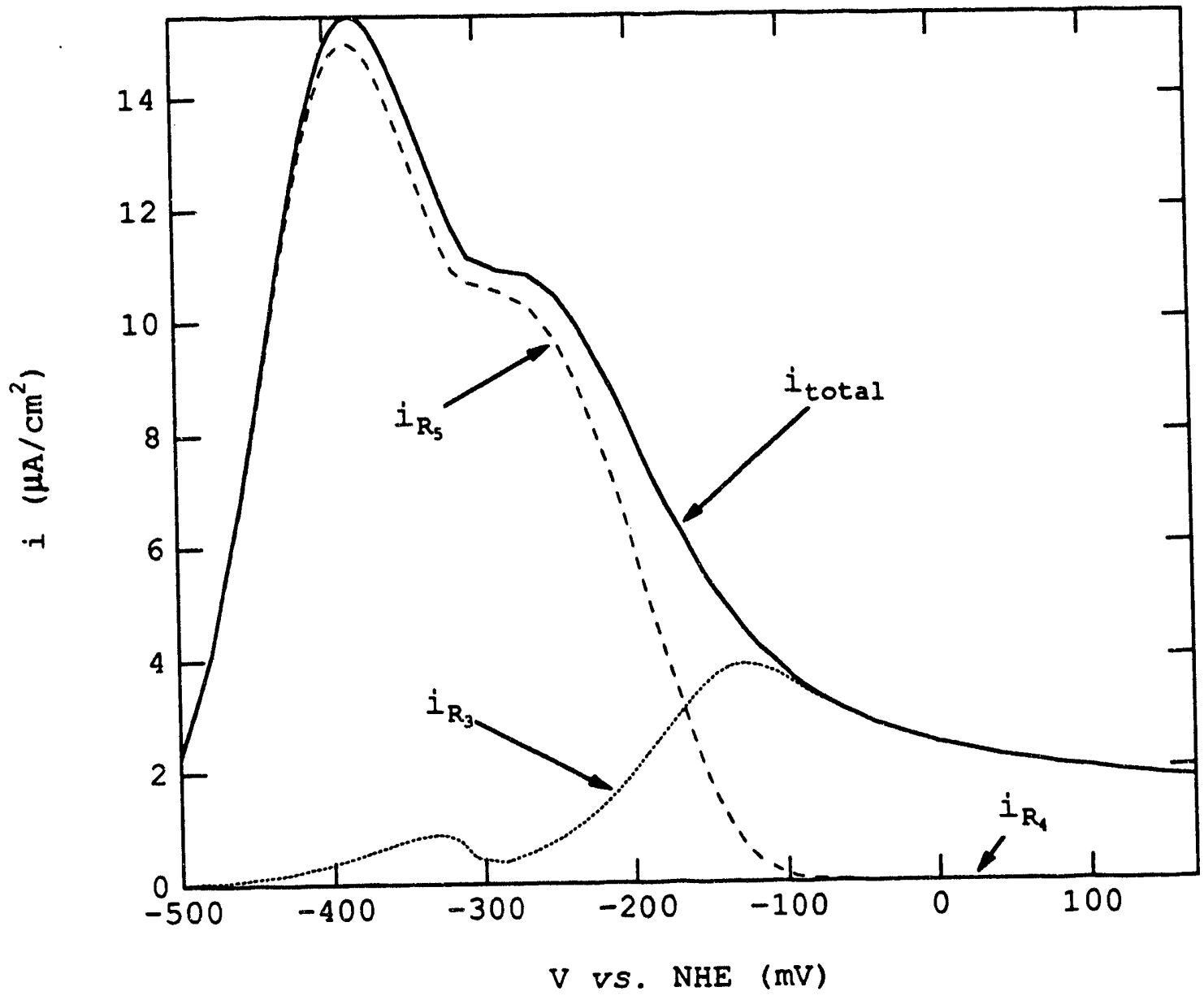

Figure 11. The currents through each reaction at the oxide/solution interface versus the potential with UPD. Sweep rate $=0.3 \mathrm{mv} / \mathrm{s}$. 
our simulations, the peak in the simulated current appears at too low a potential as compared to the peak in the current in the experimental data. If we-attempted to match the peak in the current with our simulations at the correct potentials, we would have to delay the UPD $200^{\circ} \mathrm{mV}$ and allow the length to reach $6 \mathrm{~nm}$ before leveling off. This would allow sufficient film growth to occur to bring the masstransfer-limited current down to the proper magnitude at the first minimum. A growing oxide that briefly levels off at $6 \mathrm{~nm}$ before continuing to grow again can not be explained by UPD. We conclude that the two events in the data are mutually exclusive. With the present amount of physics included in our analysis, it is impossible for us to match all of the data provided by Jovancicevic et al.

A final comparison to experimental data is provided. Included in figure 12 are the data from Jovancicevic et al., our original simulation (without UPD), and the current versus potential curve reproduced from Lukac et al. 25 Our work appears to be in far better agreement with the work of Lukac et al. than that performed by Jovancicevic et al. After exhausting all physically reasonable avenues for creating simulations that could duplicate the results of Jovancicevic et al., we come to the conclusion that perhaps the work done by Lukac et al. was done under better controlled conditions.

\section{Summary}

In this chapter we have presented the equations that describe the growth of a film. Methods of combining the equations to elim- 


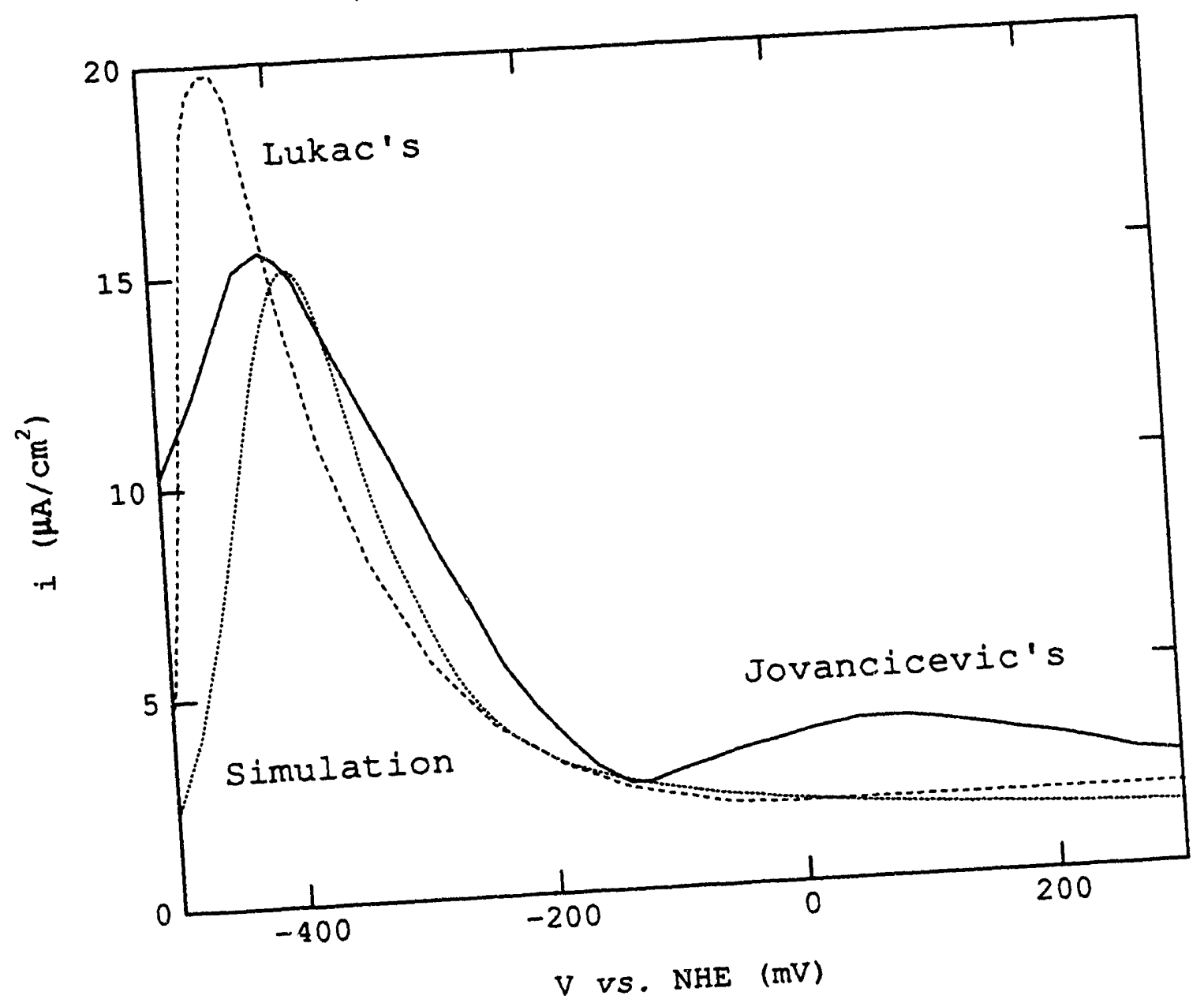

Figure 12. Current versus potential from Jovancicevic et al. and the simulation at a sweep rate of $0.3 \mathrm{mV} / \mathrm{s}$ and Lukac et al. ${ }^{25}$ at a sweep rate of $1 / 3 \mathrm{mV} / \mathrm{s}$. 
inate rapid reactions are provided, as well as the boundary conditions and a means for solving the equations. Equations that find specific application to oxide films were developed as were variables that make the computation simpler. A model was developed specifically for the iron/iron oxide system. The method by which certain parameters are determined was given. A program was then written and results of a low-field mole-flux equation were displayed. A comparison to existing experimental data and the effects of certain parameters on the final simulations were also discussed. A final comparison to experimental data showed good agreement with the work of Lukac et al. From this analysis we conclude that as the potential of iron in a borate buffer solution is swept in an increasing manner, iron initially reacts to form dissolved ferrous species. Eventually, at approximately $200 \mathrm{mV}$ above equilibrium, an oxide is formed that is thick enough to cause a switch to the mass-transfer-limited regime and the onset of passivation. At higher potentials, the oxide-filmforming reaction becomes the dominant reaction.

\section{List of Symbols}

Roman

$\begin{array}{ll}a & \text { jump distance, } \mathrm{cm} \\ a_{k} & \text { activity of species } \mathrm{k} \\ c_{k} & \text { concentration of species } \mathrm{k}, \mathrm{mol} / \mathrm{cm}^{3} \\ D_{k} & \text { diffusion coefficient of species } \mathrm{k}, \mathrm{cm}^{2} / \mathrm{s}\end{array}$




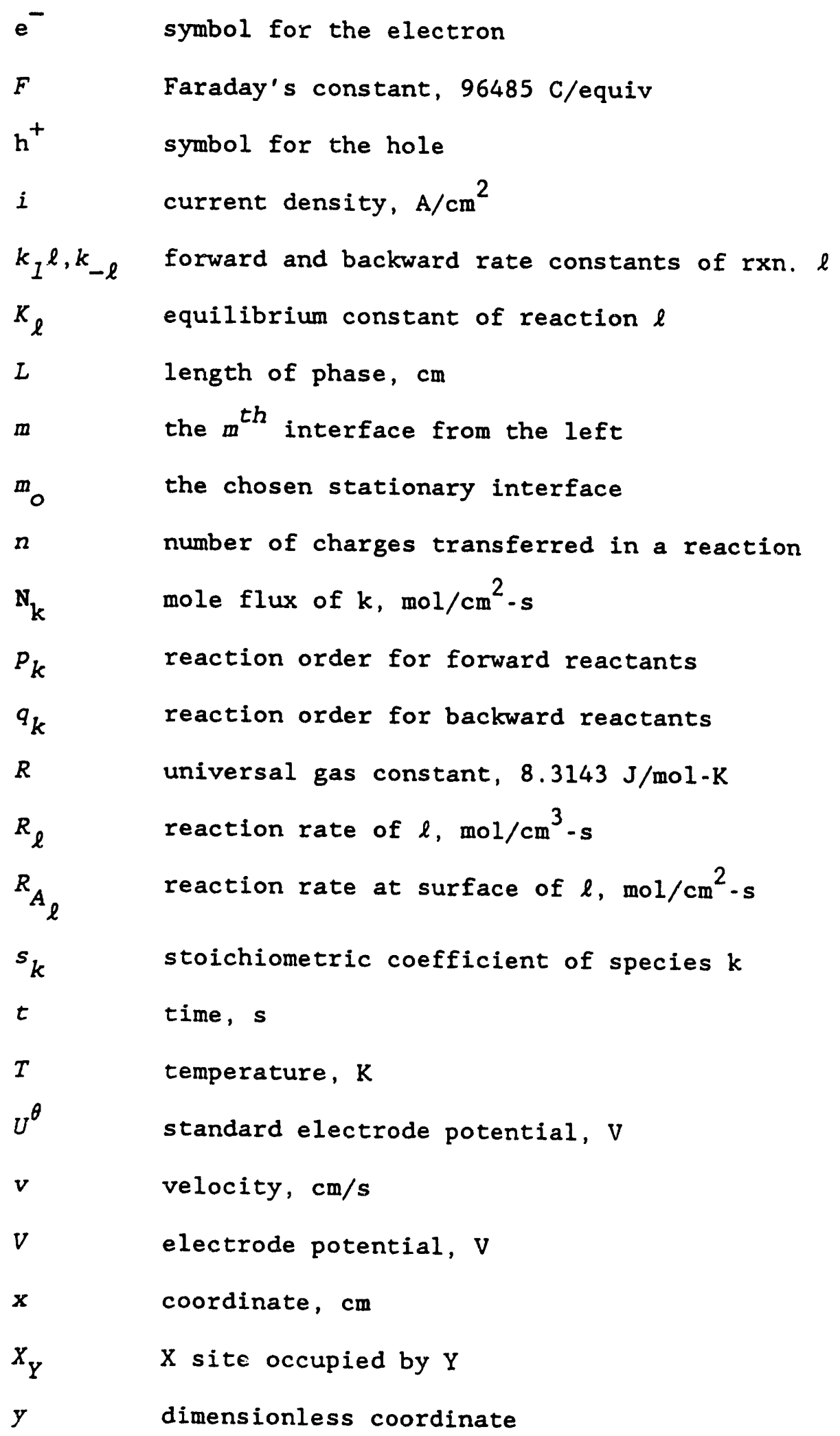




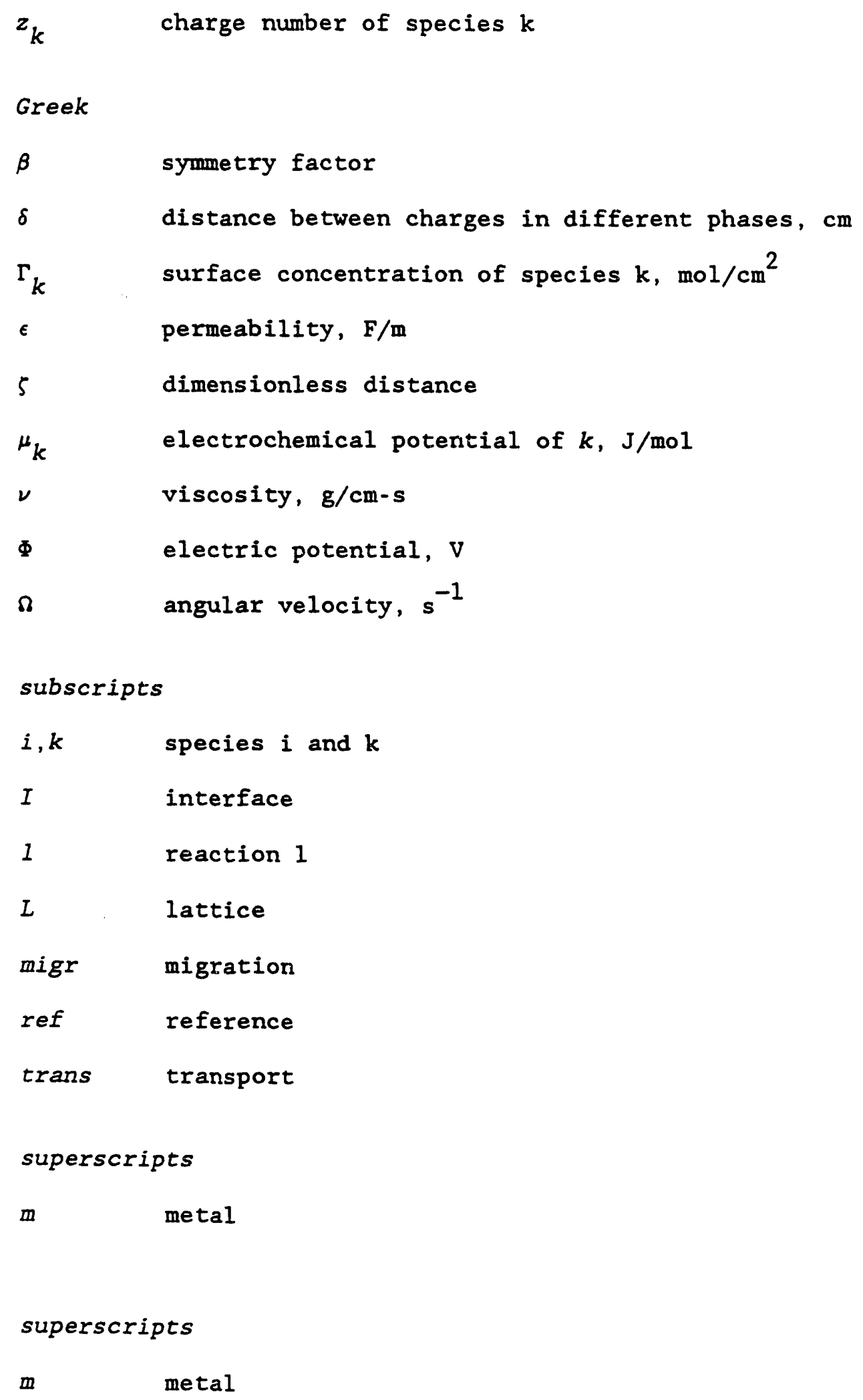




oxide
solution
position to the left of $x$
position to the right of $x$

\section{References}

1. L. Young, "The Theory of Formation of High Resistance Anodic Oxide Film," Can. J. Chem., 371, 276-285 (1959).

2. L. Young, Anodic Oxide Films, Academic Press, London (1961).

3. K. J. Vetter, Electrochemical Kinetics. Theoretical and Experimental Aspects, Academic Press, New York, 748-789 (1967).

4. C. Y. Choa, L. F. Lin, and D. D. Macdonald, "A Point Defect Model for Anodic Passive Films," J. Electrochem. Soc., 128, 1186-1194 (1981).

5. E. J. W. Verwey, "Electrolytic Conduction of a Solid Insulator at High Fields," Physica, 2, 1059-1063 (1935).

6. N. Cabrera and N. F. Mott, "Theory of the Oxidation of Metals," Repts. Progr. Phys., 12, 163-184 (1948-49).

7. F. P. Fehlner and N. F. Mott, "Low-Temperature Oxidation," Oxidation of Metals, 2, 59-99 (1970). 
8. R. J. Maurer, "Deviations from Ohm's Law in Soda Lime Glass", J. Chem. Phys., 9, 579-584 (1941).

9. D. A. Vermilyea, "The Kinetics of Formation of Anodic Tantalum Oxide - Further Studies," J. Electrochem. Soc., 102, 655-659 (1955).

10. G. T. Burnstein and A. J. Davenport, "The Current-Time Relationship during Anodic Oxide Film Growth under High Electric Field," J. Electrochem. Soc., 136, $936-941$ (1989).

11. B. D. Cahan and C.-T. Chen, "The Nature of the Passive Film on Iron. III. The Chemi-Conductor Model and Further Supporting Evidence," J. Electrochem. Soc., 129, 921-925 (1982).

12. C. P. Bean, J. C. Fisher, and D. A. Vermilyea, "Ionic Conductivity of Tantalum Oxide at Very High Fields," Physical Review, $101,2,551-554$ (1956).

13. L. L. Odynets, "Ionic Transport in Anodic Oxide Films in Strong Fields," Elektrokhimiya, 26, 1040-1043 (1990).

14. J. F. Dewald, "The Effects of Space Charge on the Rate of Formation of Anode Films," Acta Met., 2, 340-341 (1954).

15. J. F. Dewald, "A Theory of the Kinetics of Formation of Anode Films at High Fields," J. Electrochem. Soc., 102, 1-6 (1955).

16. M. J. Dignam, "Transition Layer Model of the OxideElectrolyte Interface," Can. J. Chem., 56, 595-605 (1978). 
17. C. J. Greyling, I. A. Kotze, and P. E. Viljoen, "The Kinetics of Oxide Film Growth on Maraging Steel as Described by Spacecharge Effects," Surf. Interface Anal., 16, 293-298 (1990).

18. N. Sato and M. Cohen, "The Kinetics of Anodic Oxidation of Iron in Neutral Solution," J. Electrochem. Soc., 111, 512-522 (1964).

19. B. E. Conway, B. Barnett, H. Angerstein-Kozlowska, and B. V. Tilak, "A Surface-Electrochemical Basis for the Direct Logarithmic Growth Law for the Initial Stages of Extension of Anodic Oxide Films Formed at Noble Metals," J. Chem. Phys., 93, 8361-8373 (1990).

20. J. Frenke1, "tber die Warmebewegung in festen end flüssigen Körpern," Z. f. Physik, 35, 652-669 (1926).

21. D. D. Macdonald and M. Urquidi-Macdonald, "Theory of Steady-State Passive Films," J. Electrochem. Soc., 137, 2395-2402 (1990).

22. D. D. Macdonald, S. R. Biaggio, and H. Song, "Steady-State Passive Films. Interfacial Kinetic Effects and Diagnostic Criteria," J. Electrochem. Soc., 139, 170-177 (1992).

23. B. MacDougall, "Role of Oxide Defects in the Anodic Oxidation of Nickel," J. Electrochem. Soc., 127, 789-795 (1980).

24. B. MacDougal1, "Interpretation of Logi - Logt Relationships for Nickel Passivation," J. Electrochem. Soc., 130, 114-117 (1983). 
25. G. Dagan and M. Tomkiewicz, "Passivation of Permalloy Thin Films. I. Kinetics of Anodic Oxidation in Neutral Solutions," $\mathrm{J}$. Electrochem. Soc., 139, 461-466 (1992).

26. C. Lukac, J. B. Lumsden, S. Smialowska, and R. W. Staehle, "Effects of Temperature on the Kinetics of Passive Film Growth on Iron," J. Electrochem. Soc., 122, 1571-1579 (1975).

27. P. Russell and J. Newman, "Anodic Dissolution of Iron in Acidic Sulfate Electrolytes. I. Formation and Growth of a Porous Salt Film," J. Electrochem. Soc., 133, 59-69 (1986).

27. C. Wagner, "The Electrochemistry of Ionic Crystals," J. Electrochem. Soc., 99, 346C-354C (1952).

28. P. G. Shewmon, Diffusion in Solids, McGraw-Hill, New York (1963).

29. 0. T. Sørensen, Nonstoiciometric Oxides, Academic Press, Inc., New York, New York (1981).

30. J. Newman, Electrochemical Systems, 2nd Edition, PrenticeHall Inc., Englewoods Cliffs, NJ (1991).

31. A. Acrivos, "The Asymptotic Form of the Laminar BoundaryLayer Mass-Transfer Rate for Large Interfacial Velocities," $J$. of Fluid Mechanics, 12, 337-357 (1962).

32. P. P. Russell, "Corrosion of Iron: The Active - Passive Transition and Sustained Electrochemical Oscillations, "Ph.D. Thesis, 
University of California, Berkeley (1984).

33. Y.- T. Chin and B. D. Cahan, "An Ellipsometric Spectroscopic Study of the Passive Film on Iron-Potential and Chloride Ion Dependence," J. Electrochem. Soc., 139, 2432-2442 (1992).

34. M. Hänisch and A. Otto, "Differential Reflection Spectroscopy of Passive Films on Iron," $J$. of Electroanalytical Chem. and Inter. Electrochem.," 308, 113-126 (1991).

35. J. Gui and T. M. Devine "Obtaining Surface-Enhanced Raman Spectra from the Passive Film on Iron," J. Electrochem. Soc., 138, $1376-1384$ (1991).

36. M. Kerkar, J. Robinson, and A. J. Forty, "In-Situ Structural Studies of the Passive Film on Iron and Iron/Chromium Alloys using X-Ray Absorption Spectroscopy," Faraday. Dis. of the Chem. Soc., 89, 31-40 (1990).

37. C. Wagner, "Models for Lattice Defects in Oxide Layers on Passivated Iron and Nickel," Ber. Bunsenges. Phys. Chem., 77, 1090 1097 (1973).

38. V. Jovancicevic, R. C. Kainthla, Z. Tang, B. Yang, and J. O'M. Bockris, "The Passive Film on Iron: An EllipsometricSpectroscopic Study," Langmuir, 3, 388-395 (1987).

39. M. Pourbaix, Atlas of Electrochemical Equilibria in Aqueous Solutions," National Association of Corrosion Engineers, Houston, 
Texas, 313-317 (1974).

40. U. F. Franck and K. G. Weil, "Zur Korrosion despassiven Eisens in Schwefelsäure, " Z. f. Elecktrochemie, 56, 814-822 (1952).

41. R. C. Weast, CRC Handbook of Chemistry and Physics, Edition 59, CRC Press, Inc., Boca Raton, Florida (1978-1979).

42. W. Schmickler and J. W. Schultze, "Electron Transfer Reactions on Oxide-Covered Metal Electrodes," Modern Aspects pf Electrochemistry, Edited by J. O'M. Bockris, B. E. Conway, and R. E. White, Plenum Press, New York, 17, 357-410 (1986).

43. C. G. Hill Jr., An Introduction to Chemical Engineering Kinetics \& Reactor Design, John Wiley \& Sons, Inc., New York (1977).

\section{Appendix}

\section{Program}

This section contains the computer program that was used to create the graphs and analysis of the iron/iron oxide system of the main text. We shall briefly describe the tasks performed by the main program, its subroutines, and functions; further information is provided within the program.

Main program iren: initializes the variables and parameters.

Subroutine autoband: puts the equations in computational differentiation form. 
Subroutine eqn: decides which equations shall be used to describe the phenomena that occur at the particular mesh point.

Subroutine eqninj: contains the equations that describe the conditions at the first and last mesh points.

Subroutine eqni: contains the equations that describe the conditions at the interfaces.

Subroutine eqnj: contains the equations that describe the conditions within a given phase.

Subroutine eqnprod: combines mass balance equations such that each of the reactions is present in only one equation.

Subroutine band: puts the parameters of equations into a banded matrix and also solves the inverted matrix returning the solution in matrix "c."

Subroutine matinv: inverts matrices.

Subroutine matmul: matrix multiplier.

Subroutine eqlbr: adds the logarithm of the equilibrium constant to the sum of the logarithm of the concentrations of an equilibrium reaction.

Subroutine smslnc: sums the stoichiometric coefficient times the logarithm of the concentrations of a reaction.

Subroutine $r f b$ : calculates the forward and backward parts of the rate constants that include the $k^{\prime} s$ and the product of the concentrations raised to their stoichiometric coefficients. 
Function fnrt: calculates the charge transfer number of a reaction and multiplies it by $F / R T$.

Function flxhfpt: calculates the diffusive and migrative flux of a species at a half mesh point.

Function accum: calculates the stretching term added to fluxes.

Following this program is a data file, "iren.dat," which was read by the main program and used to generate some of the data produced for the iron/iron oxide system. 


\section{Main Program}

$$
\text { iren }
$$

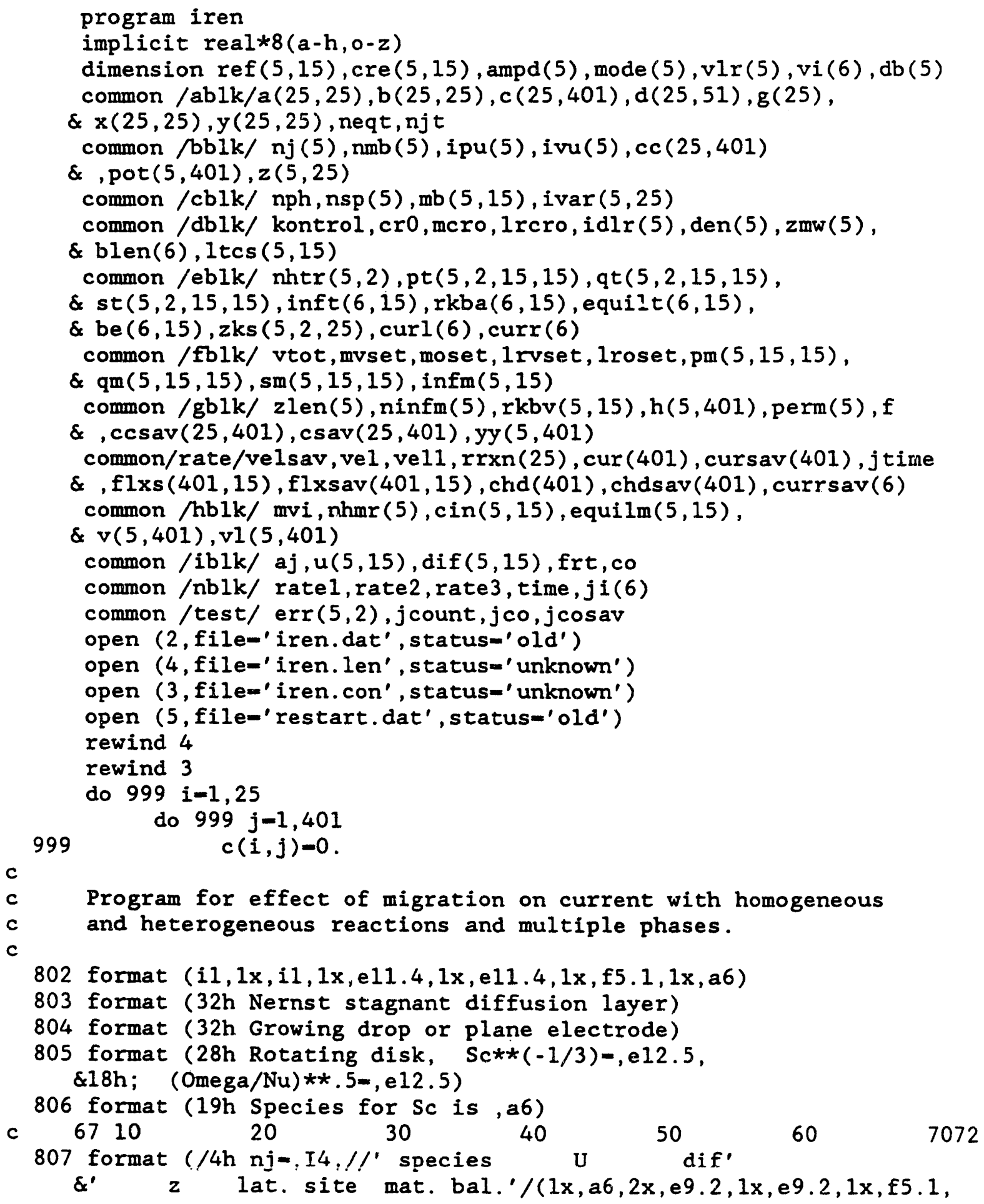


$\& 6 \mathrm{x}, \mathrm{il}, 9 \mathrm{x}, \mathrm{il}))$

808 format $(/ 8 \mathrm{x}, 31 \mathrm{~h} \mathrm{~s}(\mathrm{i}, 1)$ Heterogeneous Reactions)

809 format $(1 \mathrm{x}, \mathrm{a} 6,5(\mathrm{f5} .1))$

810 format ( $/ 8 \mathrm{x}, 29 \mathrm{~h} s(i, 1)$ Homogeneous Reactions)

812 format ( $/ 38 \mathrm{~h}$ The next run did not converge, jkon3-,i4)

813 format ( $/ 31 \mathrm{~h}$ species left right)

814 format ( $1 \mathrm{x}, \mathrm{a} 6,2 \mathrm{x}, 2 \mathrm{e} 12.5)$

820 format $(/ 3 \mathrm{x}, \mathrm{a} 6,12 \mathrm{~h}$ cur. den. $=, \mathrm{e} 12.5,17 \mathrm{~h}(\mathrm{~mA} / \mathrm{cm} * * 2)$ amp $=, e 12.5)$

821 format ( $/ 16 \mathrm{~h}$ cur. den. set $=, e 12.5,6 \mathrm{~h}$ it is, e12.5,8hin phase $\&, i 1,7 \mathrm{~h}$ to the, il, 16h(1-left, 2-right))

822 format ( $/ 22 \mathrm{~h}$ The total potential $-, e 12.5,3 \mathrm{~h} \mathrm{mv}$ )

823 format ( $/ 19 \mathrm{~h}$ Potential is set $-, e 12.5,15 \mathrm{~h} \mathrm{mv}$ cur.den. =, \&e12.5,11h (mA/cm**2))

824 format ( $/ 13 \mathrm{~h}$ The conc. of $, a 6,28 \mathrm{~h}$ is set. Potential is set = $\&, e 12.5,24 \mathrm{~h} \mathrm{mv}$, and the cur. den. $=, e 12.5,9 \mathrm{~h} \mathrm{~mA} / \mathrm{cm} * * 2$ )

830 format ( $/ 43 \mathrm{~h}$ Time Velocity of Interfaces)

831 format ( $1 \mathrm{x}, \mathrm{e} 11.4,2 \mathrm{x}, 6(\mathrm{e} 11.4,2 \mathrm{x})$ )

832 format ( $/ 35 \mathrm{~h}$ Time Length or Each Phase)

c

f : Faraday's constant.

frt : Faraday's constant divided by the gas constant and the absolute temperature with units of $1 / \mathrm{mV}$.

$a a, b b$ : constants describing flow to a rotating disk.

pi $=3.14159265358979$

$f=96487$.

frt $=f / 8.3143 / 298.15 / 1000$.

$\mathrm{aa}=0.51023262$

$\mathrm{bb}=-0.61592201$

Read in or define the following values.

area : area of disk.

cr0 : concentration of the limiting reactant.

ctot: total current through cell.

kontrol : indicates whether the program is to solve for

the current for a species at a set concentration at

the electrode surface, 1; the current for a set potential

at the electrode surface, 2; for the potential for a set total current, 3 .

mcro, lrcro : phase and side of phase of limiting reactant.

mvi : interface where velocity is set to zero.

moset, lroset : phase and side where potential is set to 0 .

mvset, Irvset : phase and side where potential is set to vtot.

neqt : will count the total number of equations to be solved.

njt : will count the total number of mesh points.

nph : number of phases.

ro : radius of disk.

vtot : total potential across cell.

read $(2, *)$ nph, kontrol, cro, ro 


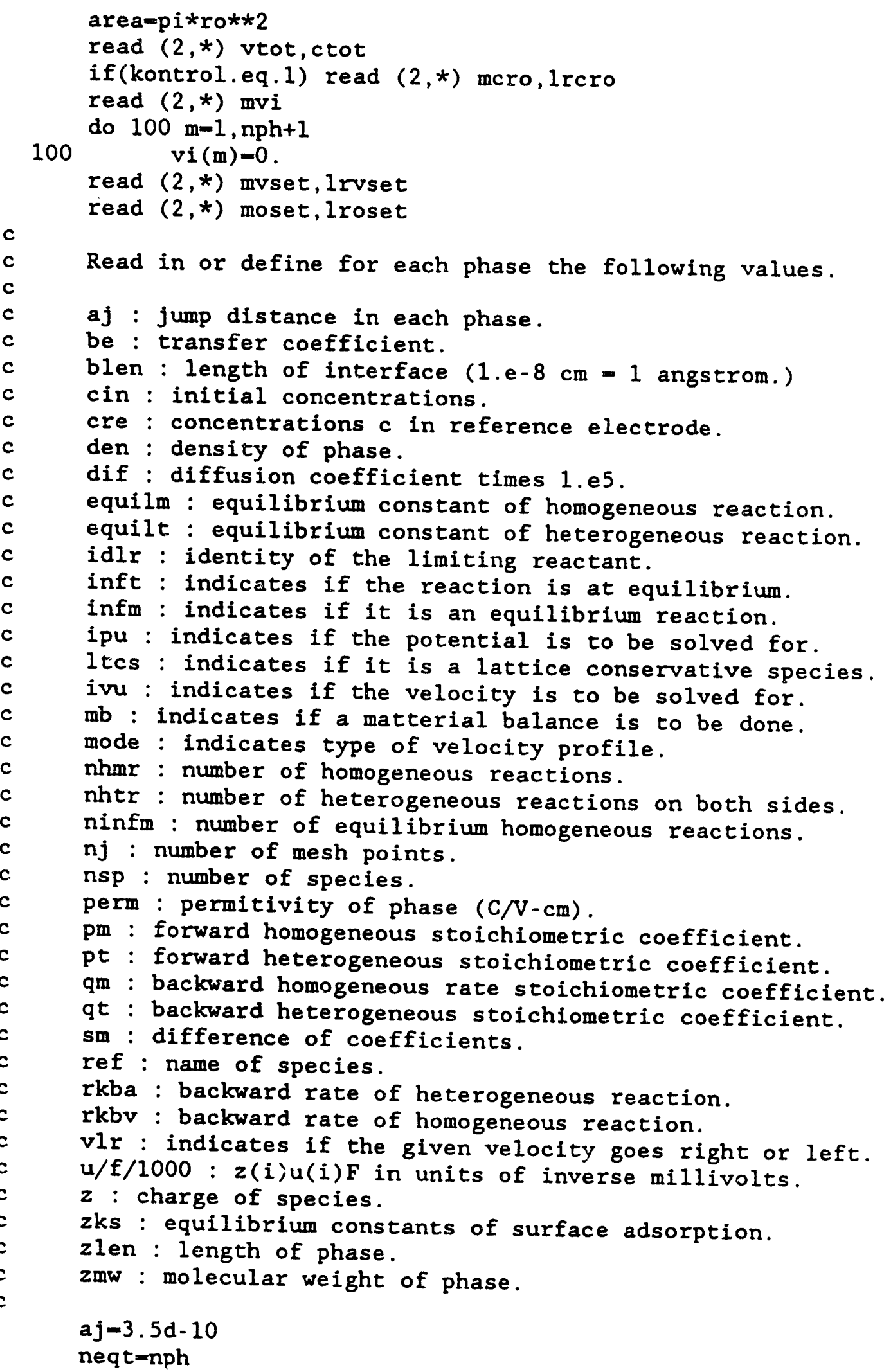




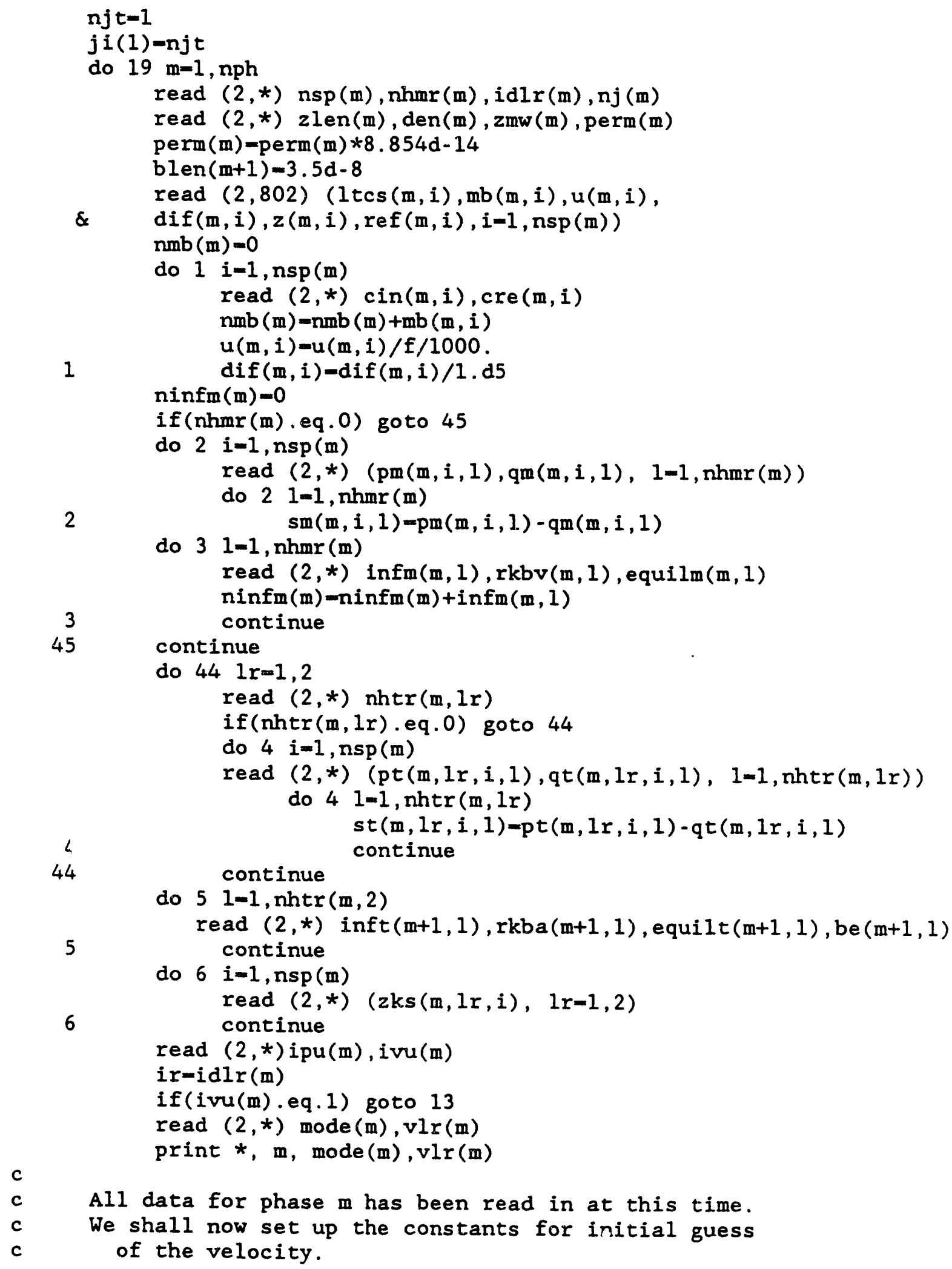


Define the following values.

ampd : limiting current with excess supporting electrolyte. anu : kinematic viscosity.

delta : order of boundary layer thickness.

difmax : maximum diffusion coefficient.

difz : diffusion coefficient of limiting reactant.

h : mesh spacing.

ir : limiting reactant.

om : rotation speed.

omnu : om/anu

scm3 : Sc number for limiting reactant to the $-1 / 3$.

time : time.

ximax : dimensionless thickness of boundary layer.

Find maximum diffusion coefficient and use for Sc number.

difmax $=0.0$

do 7 i=1, nsp (m)

if $(\mathrm{mb}(\mathrm{m}, \mathrm{i})$.eq.0) goto 7

if (dif $(m, i) \cdot g t \cdot \operatorname{difmax})$ then $\operatorname{difmax}=\operatorname{dif}(m, i)$ idz=i

7

endif continue

$\operatorname{difz}=\operatorname{dif}(m$, ir $)$

if (mode (m). ne.1) goto 9

const $=0.0$

print 803

$\operatorname{ampd}(\mathrm{m})=1.0$

$x i \max =1.0$

delta-zlen(m)

9

if (mode (m).ne.2) goto 10

const $=2.0$

print 804

$\operatorname{ampd}(\mathrm{m})=1.128379167$

$x \operatorname{imax}-3.3 *(\operatorname{difmax} / \operatorname{dif} z) * * 0.5$

read $(2, *)$ time

delta-(12.*difz*time $/ 7) * *$.

10

if (mode (m).ne. 3 ) goto 11

const $=2.0$

print 804

$\operatorname{ampd}(\mathrm{m})=1.128379167$

$x$ imax $-3.3 *$ (difmax $/$ difz $) * * 0.5$

read $(2, *)$ time

delta $(4 . *$ difz $*$ time $) * * 0.5$

11

if (mode (m).ne.4) goto 12

const $=3.0$

read $(2, *)$ anu, om

omnu=om/anu

$\operatorname{scm} 3=(\mathrm{anu} / \mathrm{dif} z) * *(-1 . / 3$.

print 805, scm3, omnu**.5 


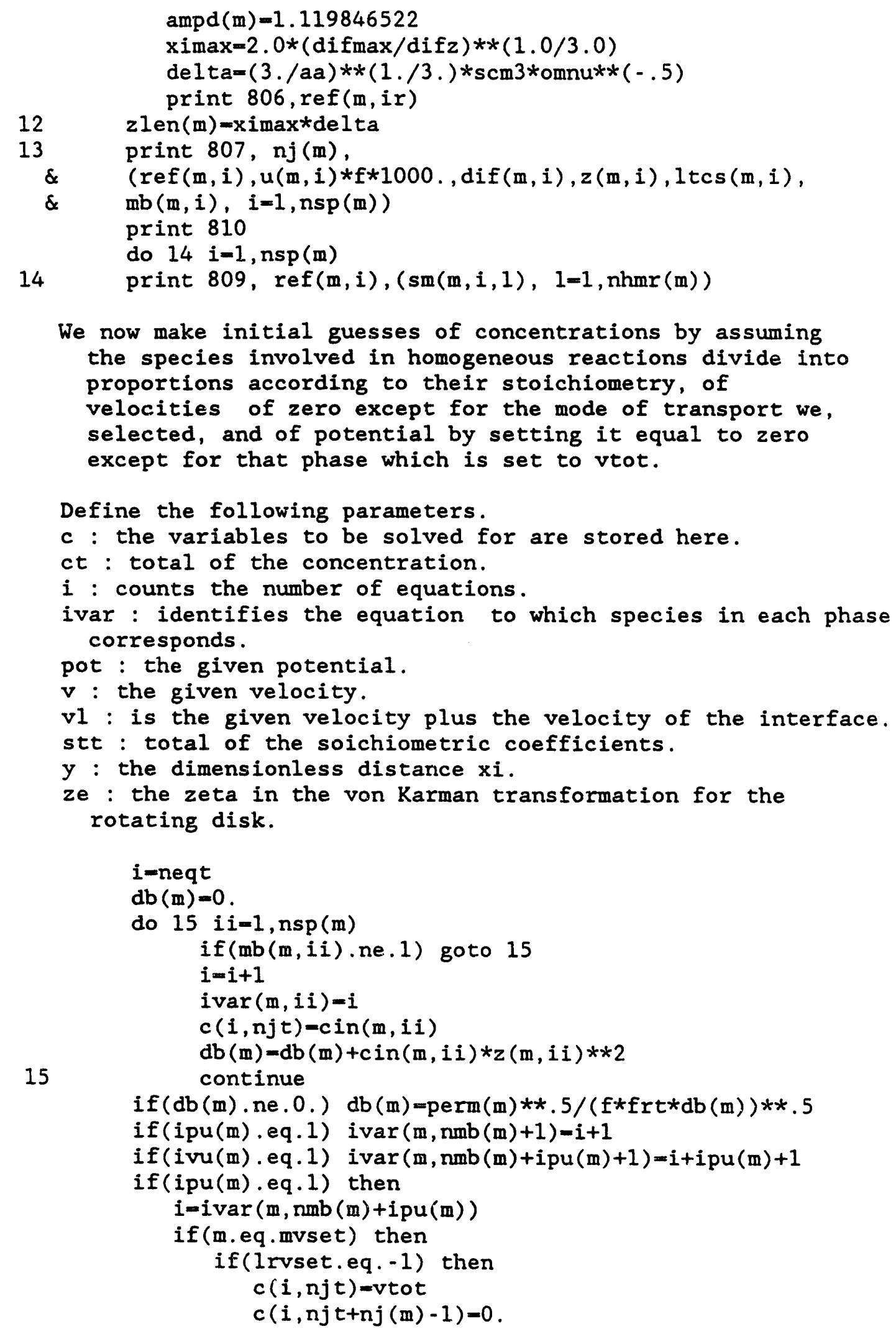

We now make initial guesses of concentrations by assuming the species involved in homogeneous reactions divide into proportions according to their stoichiometry, of velocities of zero except for the mode of transport we, selected, and of potential by setting it equal to zero except for that phase which is set to vtot.

Define the following parameters.

$c$ : the variables to be solved for are stored here.

ct : total of the concentration.

$i$ : counts the number of equations.

ivar : identifies the equation to which species in each phase corresponds.

pot: the given potential.

$v$ : the given velocity.

$v 1$ : is the given velocity plus the velocity of the interface.

stt : total of the soichiometric coefficients.

$\mathrm{y}$ : the dimensionless distance $\mathrm{xi}$.

ze : the zeta in the von Karman transformation for the rotating disk.

c 


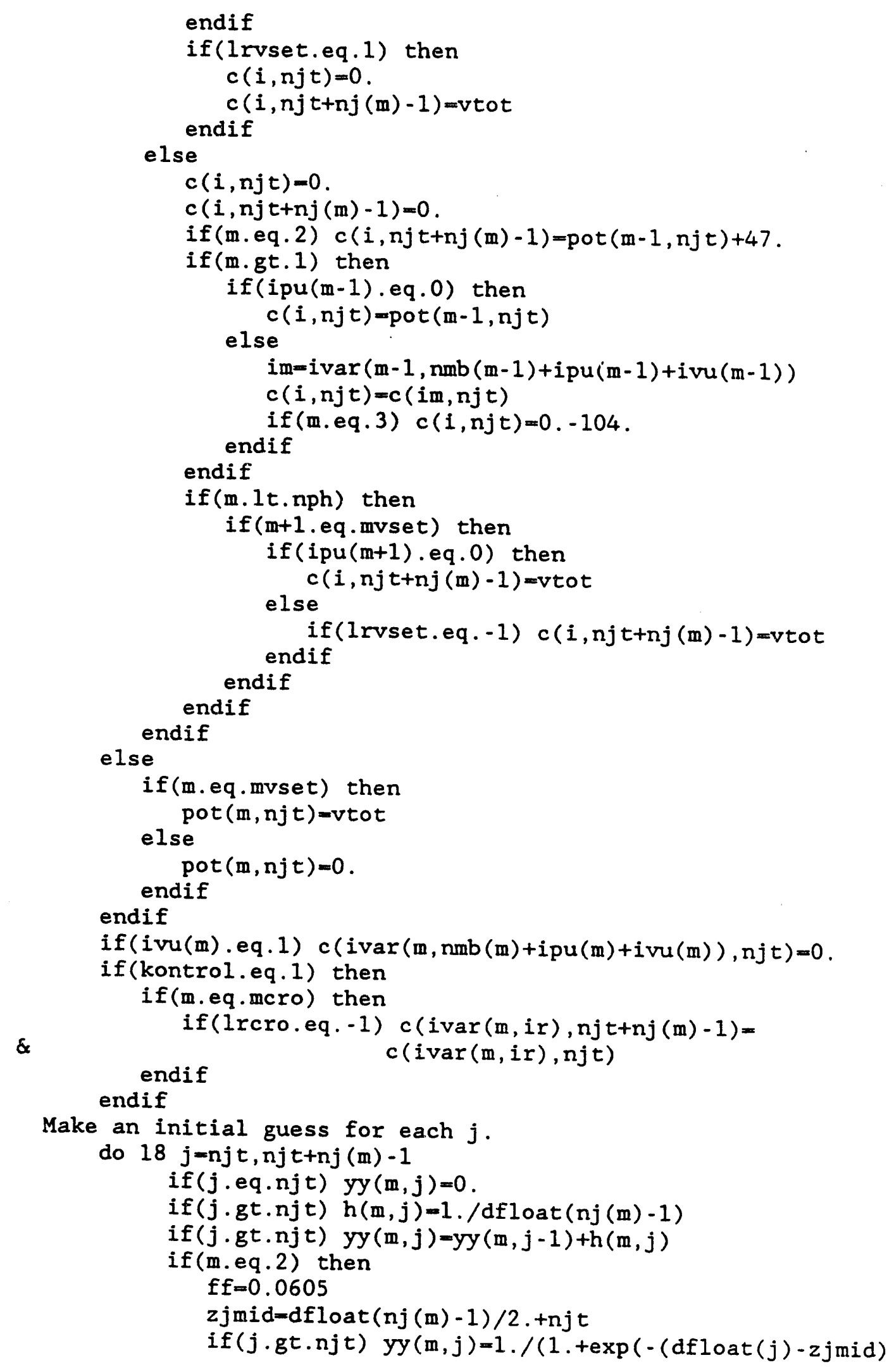


$\&$

$\varepsilon$

$\&$

$\&$

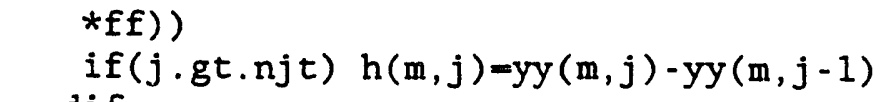

endif

if (m.eq. 3$)$ then

$f f=0.125$

if $(j \cdot g t \cdot n j t)$ yy $(m, j)=\operatorname{dexp}(\operatorname{dfloat}(j-n j t-1) * f f)$

$/ \operatorname{dexp}(\operatorname{dfloat}(n j(m)-2) * f f)$

endif

if $(j \cdot g t \cdot n j t) h(m, j)=y y(m, j)-y y(m, j-1)$

if $(j \cdot$ eq $. n j t+n j(m)-1)$ yy $(m, j)=1$.

if $(j$.eq. $n j t+n j(m)-1) h(m, j)=y y(m, j)-y y(m, j-1)$

if (ivu(m).eq.0) then

if $(\operatorname{vlr}(m) . g t .0)$ yd $=(1 .-y y(m, j)) * z \operatorname{len}(m) /$ delta

if $(v \operatorname{lr}(m) .1 t .0)$ yd=yy $(m, j) * z \operatorname{len}(m) /$ delta

$v(m, j)=v l r(m) *$ const*yd*difz/delta

if $(\operatorname{mode}(m)$.eq. 4) then

$z e-y d *(3 . / a a) * *(1 . / 3). * \operatorname{scm} 3$

$v(m, j)=v(m, j) * y d$

$*(1.0-z e / a a *(1 . / 3 .+b b / 6 . * z e+(b b * z e) * * 2 / 30$.

$+a a * z e * * 3 / 180 .-(1 .-4 . * a a * b b) * z e * * 4 / 1260)$. endif

else

$v I(m, j)=v(m, j)$

$i=i \operatorname{var}(m, n m b(m)+i p u(m)+i v u(m))$

endif

$c(i, j)=c(i, n j t)$

if (ipu(m).eq.1) then

$i=i \operatorname{var}(m, n m b(m)+i p u(m))$

if (m.eq.2) then

fctr $=(1,-y y(m, j)) *_{z} \operatorname{len}(m) / d b(m)$

if (fctr.gt.9.2) then

else $c(i, j)=c(i, n j t)$

$c(i, j)=c(i, n j t)+(c(i, n j t+n j(m)-1)-$

endif

endif

if (m.eq.3) then

$c(i, n j t+n j(m)-1)=0$.

$f c t r=y y(m, j) * z \operatorname{len}(m) / d b(m)$

if (fctr.gt.9.2) then

else $c(i, j)=c(i, n j t+n j(m)-1)$

$c(i, j)=c(i, n j t) * \operatorname{dexp}(-f c t r)$ endif

endif

else

$\operatorname{pot}(m, j)=\operatorname{pot}(m, n j t)$

endif

if (ivar (m,ir).ne.0) $c(i v a r(m, i r), j)=c(i v a r(m, i r), n j t)$ if (kontrol.eq.1) then 
$\&$

$\&$

18

19

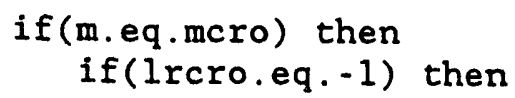

$\&$

$\&$

else

$$
, c(2, j), c(3, j),(c(i \operatorname{var}(m, i), j) \text {, }
$$

51

nj sum-nj sum+nj (m) - 1

continue

velsav--c $(2,4)$

jtime: the time stepping loop.

jkon3 : the loop when converging on a particular total total current (kontrol-3.)

time $=0$.

time tot $=1.0 \mathrm{~d} 05$

it count $=0$

potset $=+600.0$

potprint $=0.0$

swr $=+1.0$

$\mathrm{dt}=0$.

nflag $=0$

if $1 \mathrm{~g}=0$

j time $=0$ 


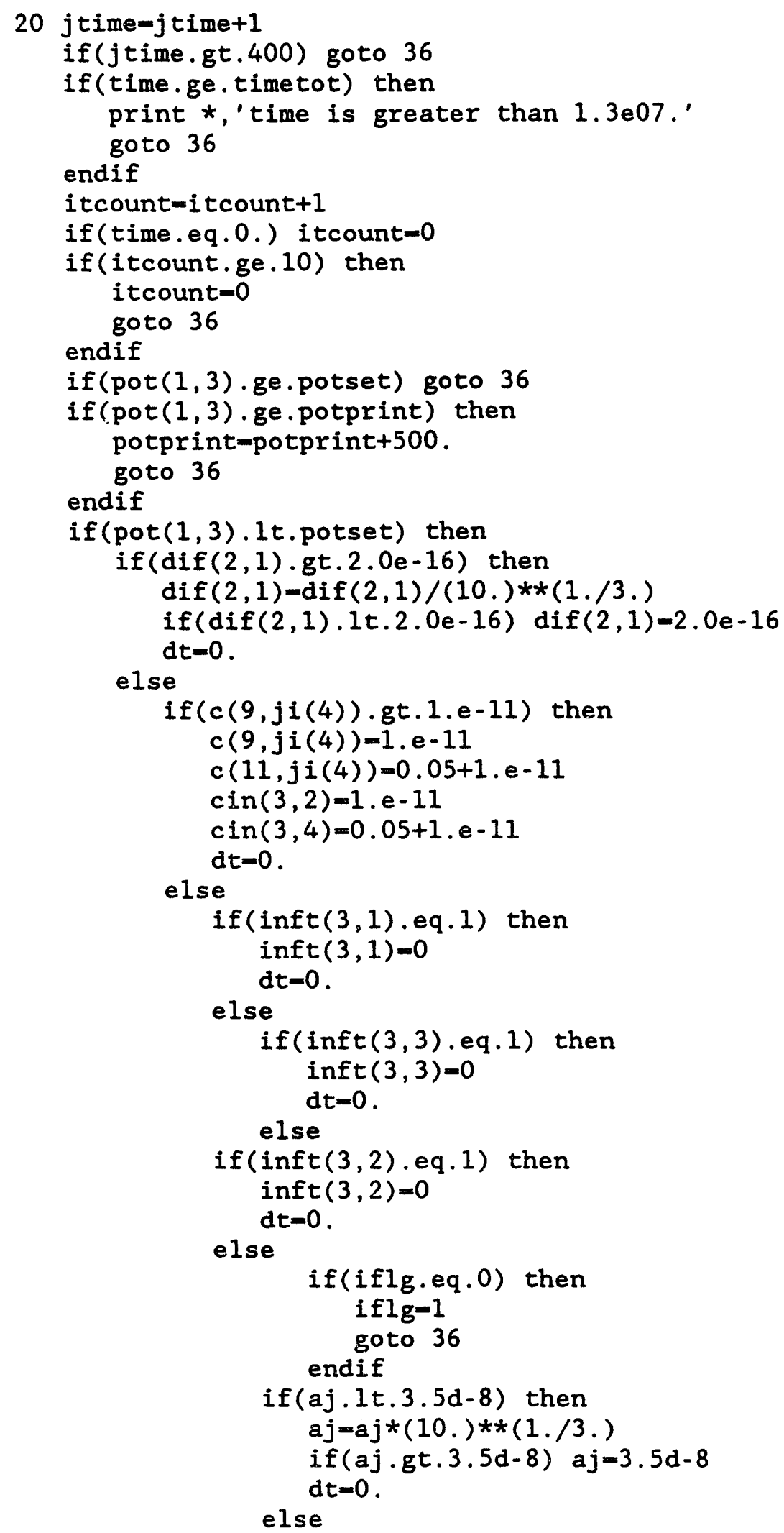


c

c

21

c

C

c

c

c

c

c

c

c if $(\operatorname{pot}(1,3) .1 t .-251.2)$ then $s w r=1$.

endif $\mathrm{dt}=1$

if (dt.gt. 70) dt $=70$.

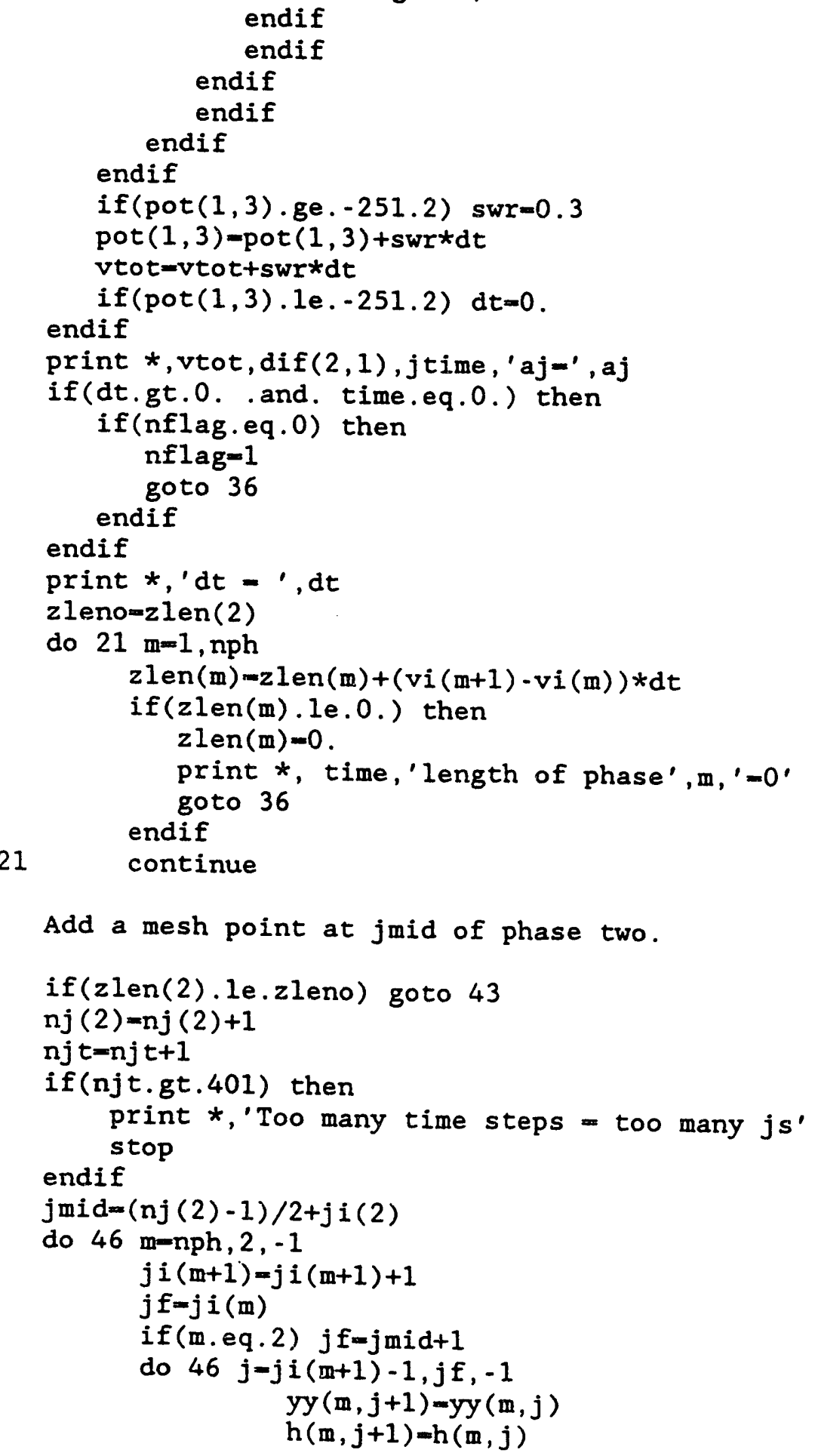

endif

print *, vtot, dif $(2,1)$, jtime, ' aj =' ,aj 
48

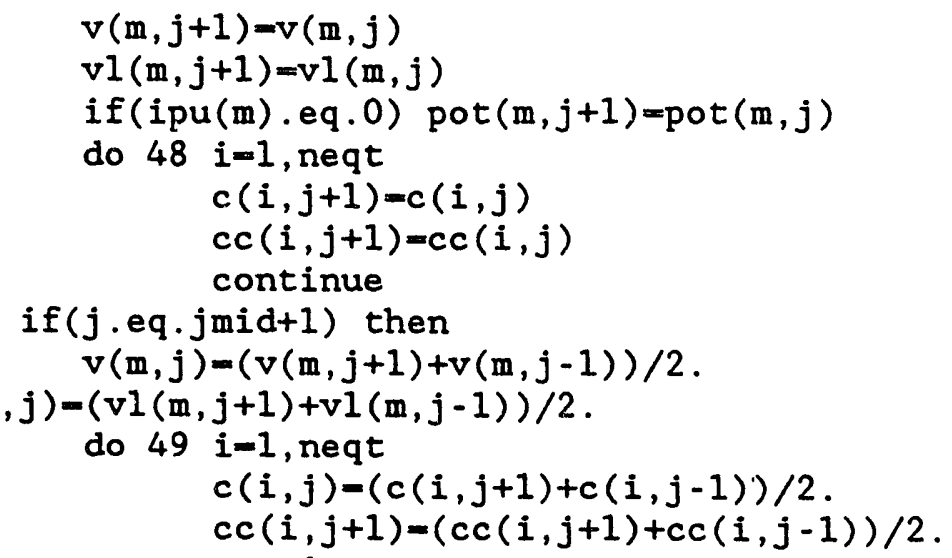




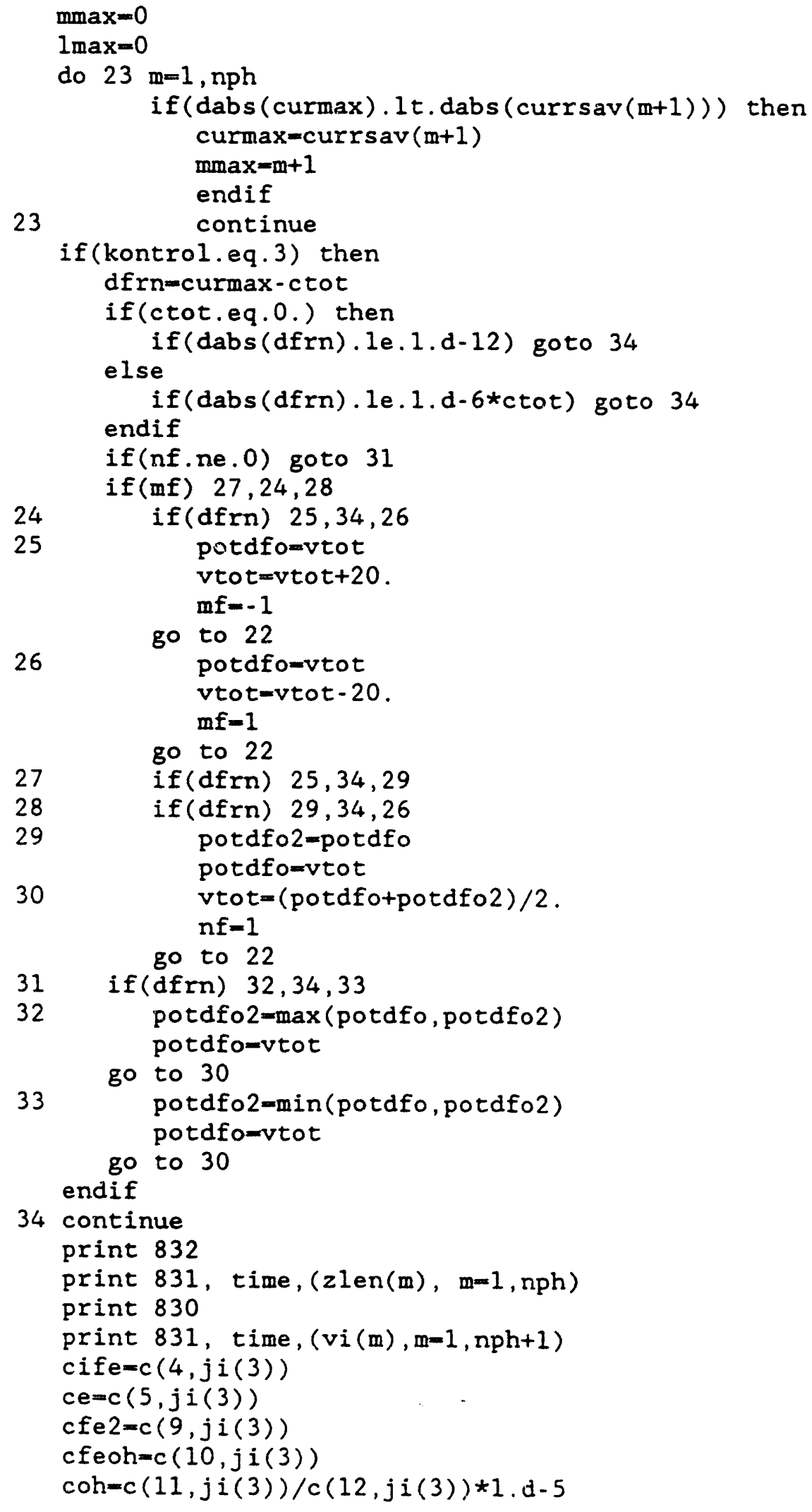




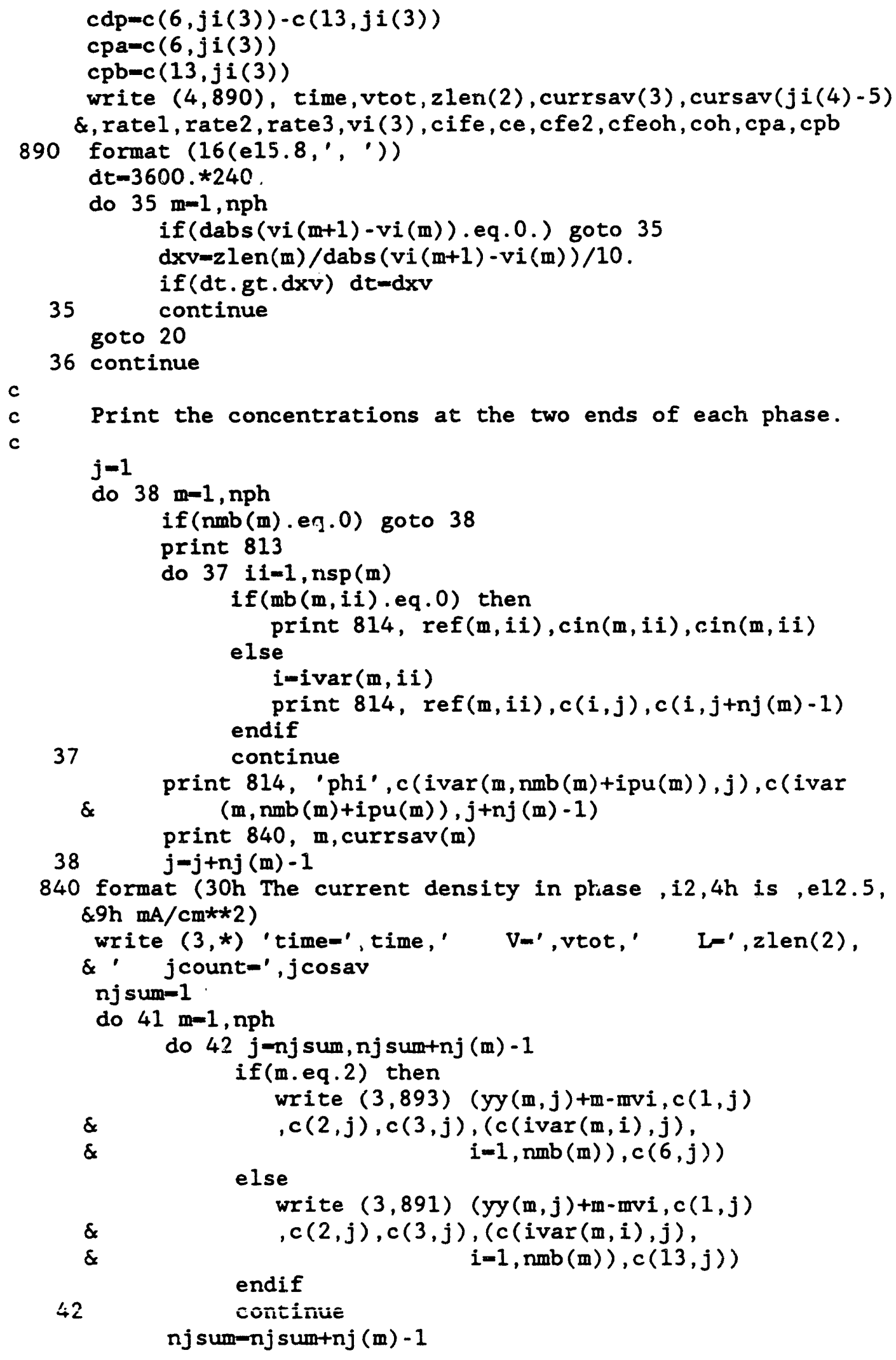




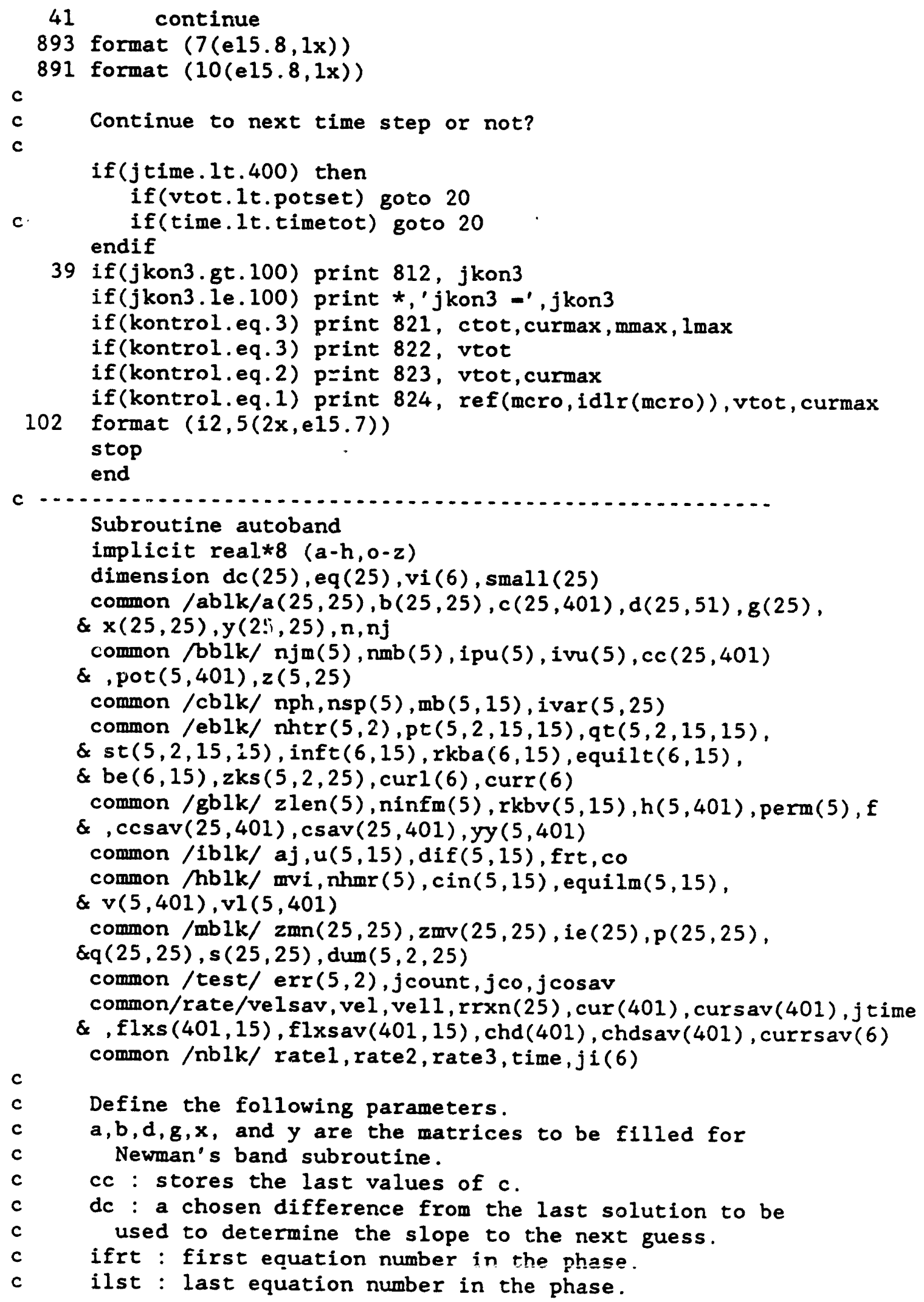




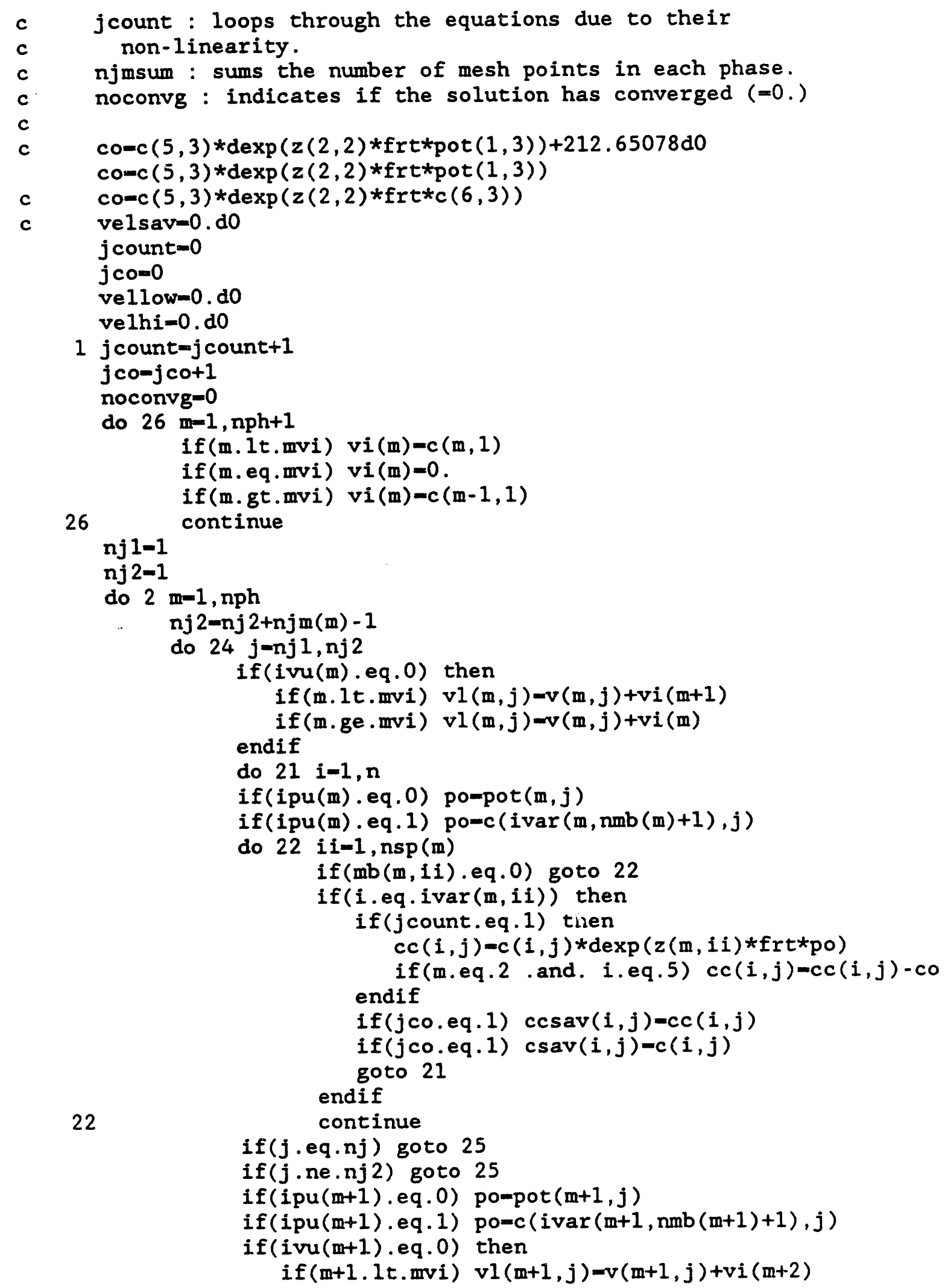




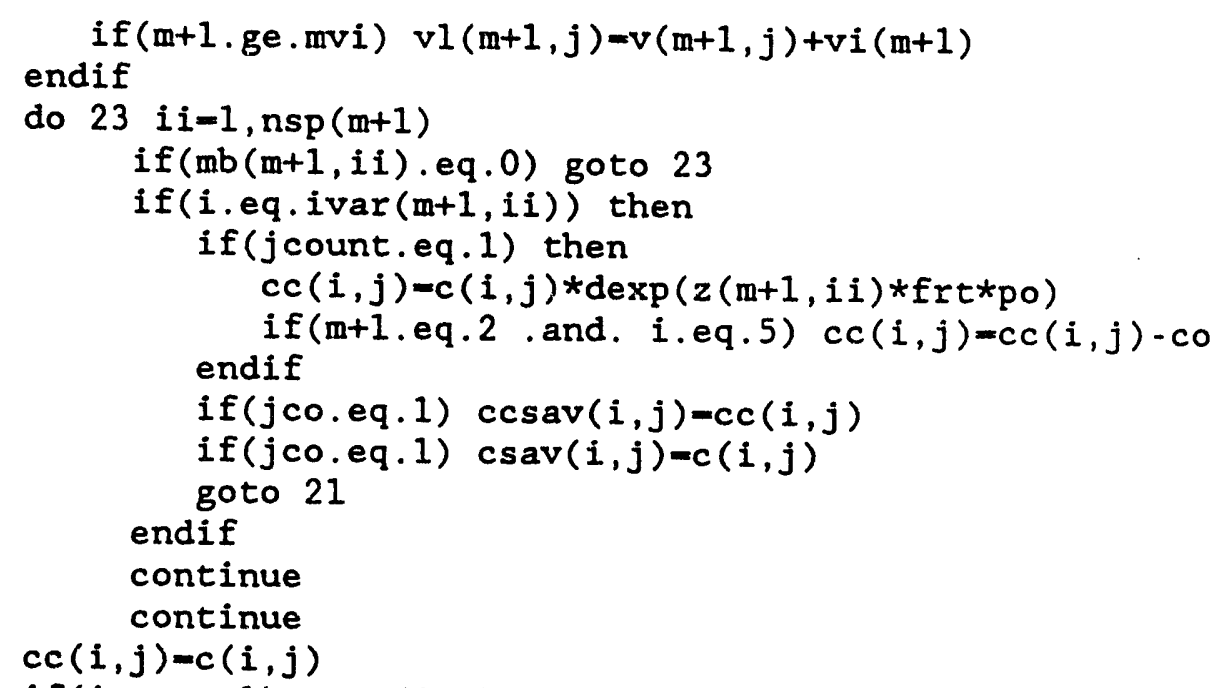




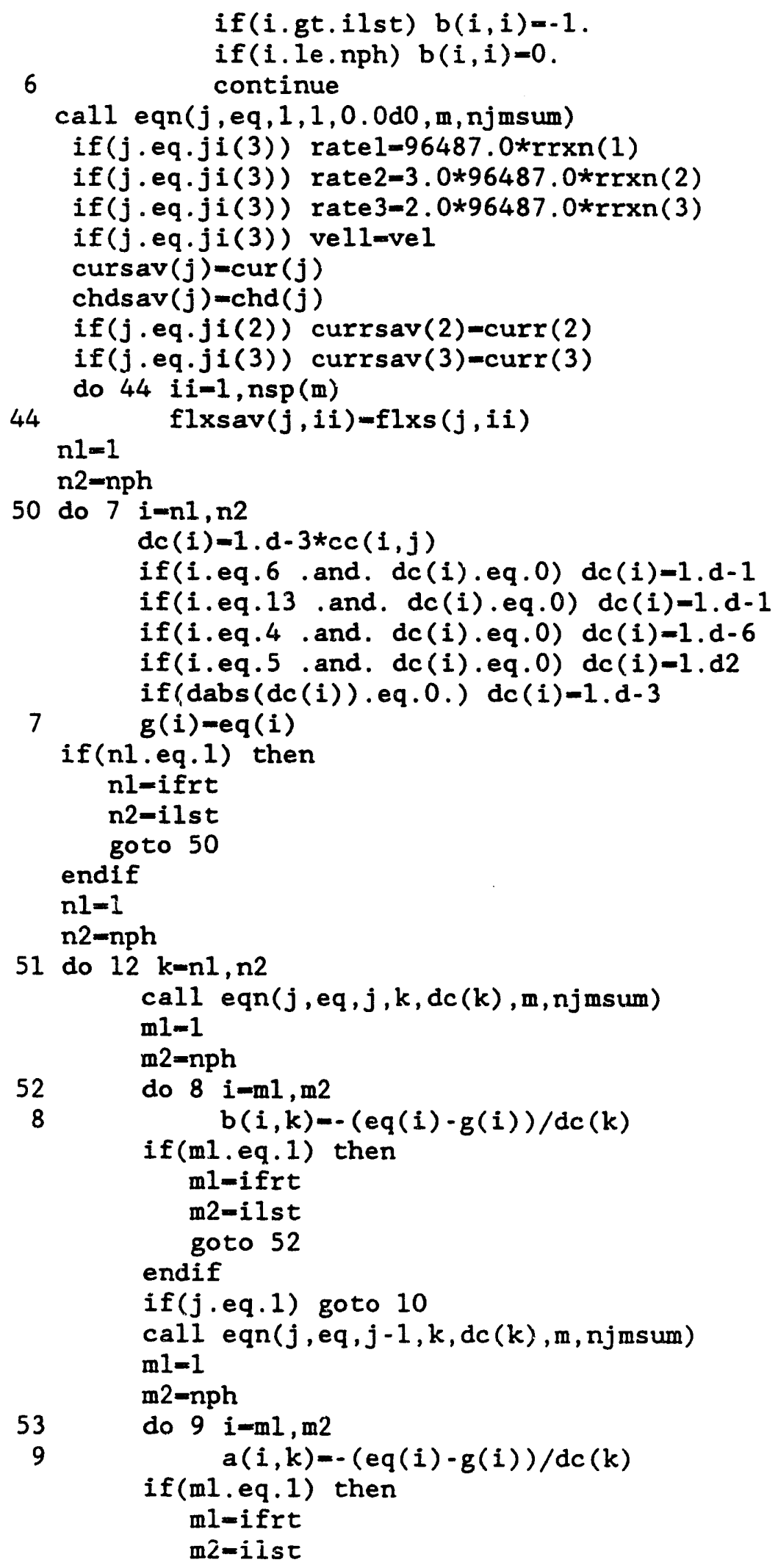




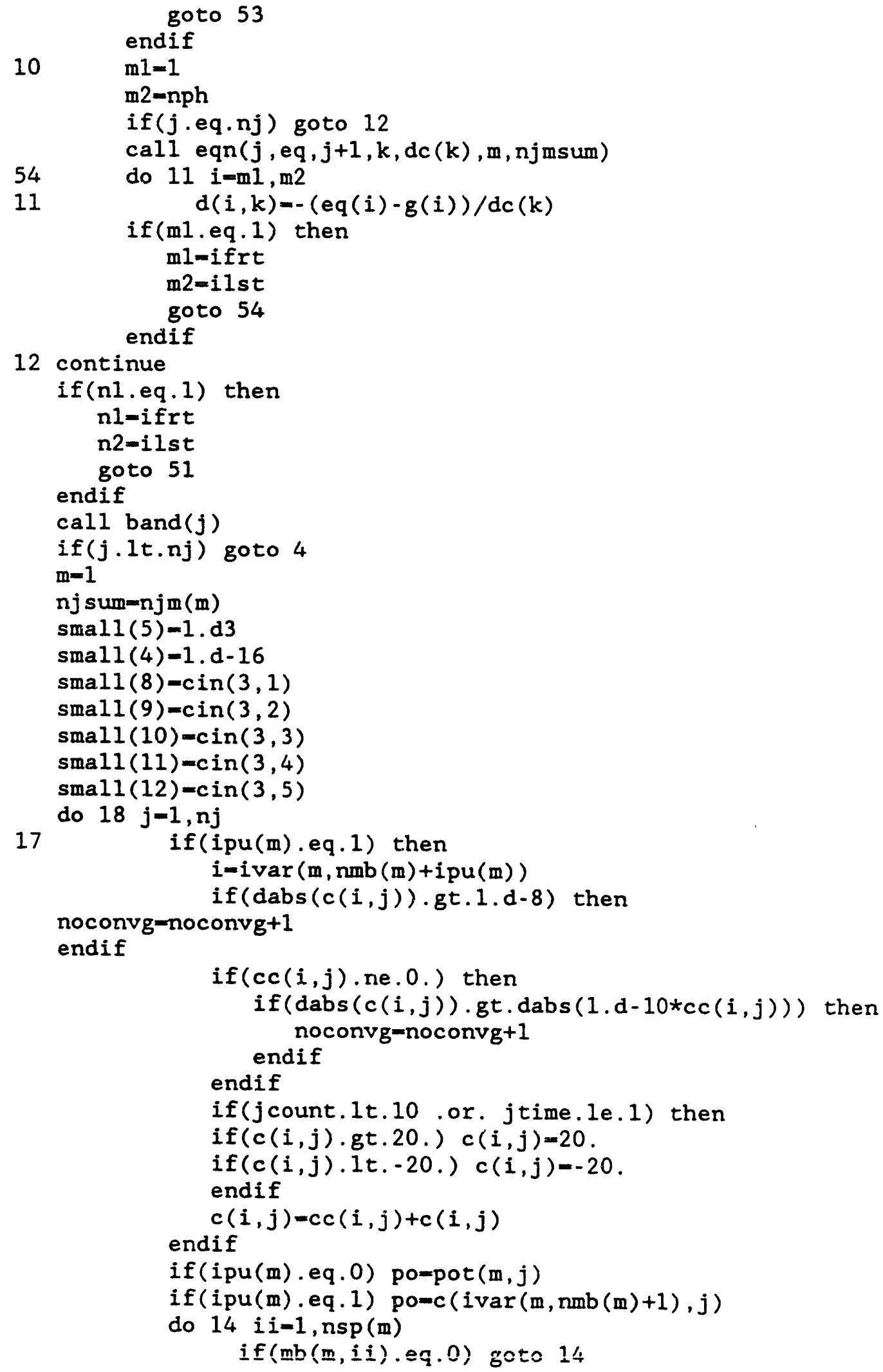


$\varepsilon$

$\varepsilon$ $i=i \operatorname{var}(m, i i)$

if $(\operatorname{cc}(i, j) . e q .0$.$) then$

if (i.eq.5) then else if $(c(i, j) .1 t .-c 0) c(i, j)=-1 . e-3 * c 0$

if $(c(i, j) .1 t .0) c.(i, j)=\operatorname{dabs}(0.001 * c(i, j))$ endif

if (dabs $(c(i, j)) \cdot g t \cdot 1 \cdot e-8)$ noconvg=noconvg+1 else

if (jcount.1t.10 .or. jtime.1e.1) then

if (i.eq.5) then

if $(c(i, j) .1 t \cdot-0.9 *(\cot c c(i, j))) c(i, j)=-.9$ $*(\operatorname{cotcc}(i, j))$

if $(c(i, j) \cdot g t \cdot 9 \cdot *(\operatorname{cotcc}(i, j))) \quad c(i, j)=9$. $*(\operatorname{cotcc}(i, j))$

else

if $(c(i, j) .1 t .-0.9 * c c(i, j)) \quad c(i, j)=-.9 * c c(i, j)$

endif

if $(c(i, j) \cdot g t \cdot 9 \cdot * c c(i, j)) c(i, j)=9 . * c c(i, j)$

endif

if (i.ne.5) then

if $(\operatorname{dabs}(c(i *, j)) \cdot g t \cdot \operatorname{dabs}(1 \cdot e-10 * c c(i, j)))$ then

noconvg-noconvg +1

endif

else

$\&$

if $(\operatorname{dabs}(c(i, j)) \cdot g t \cdot \operatorname{dabs}(1 \cdot e-10 * c c(i, j))$

endif

endif

$\&$

14

endif noconvg-noconvg+1

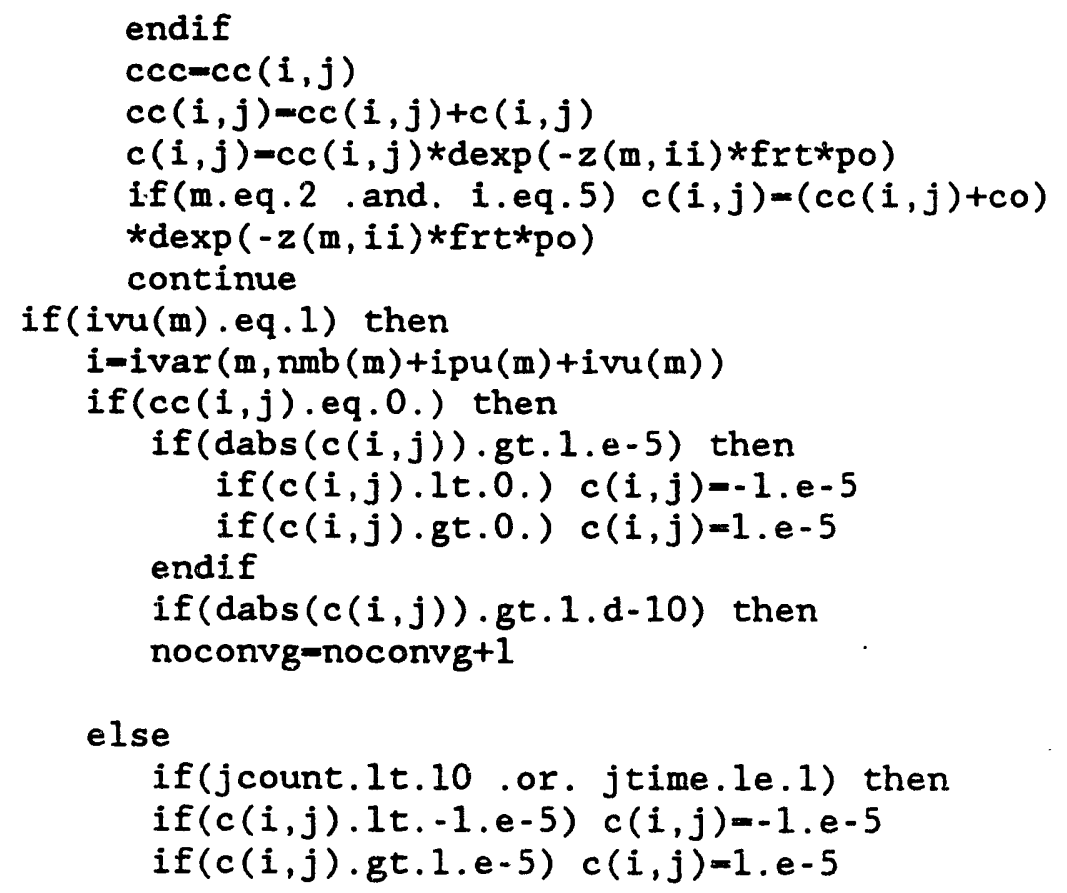




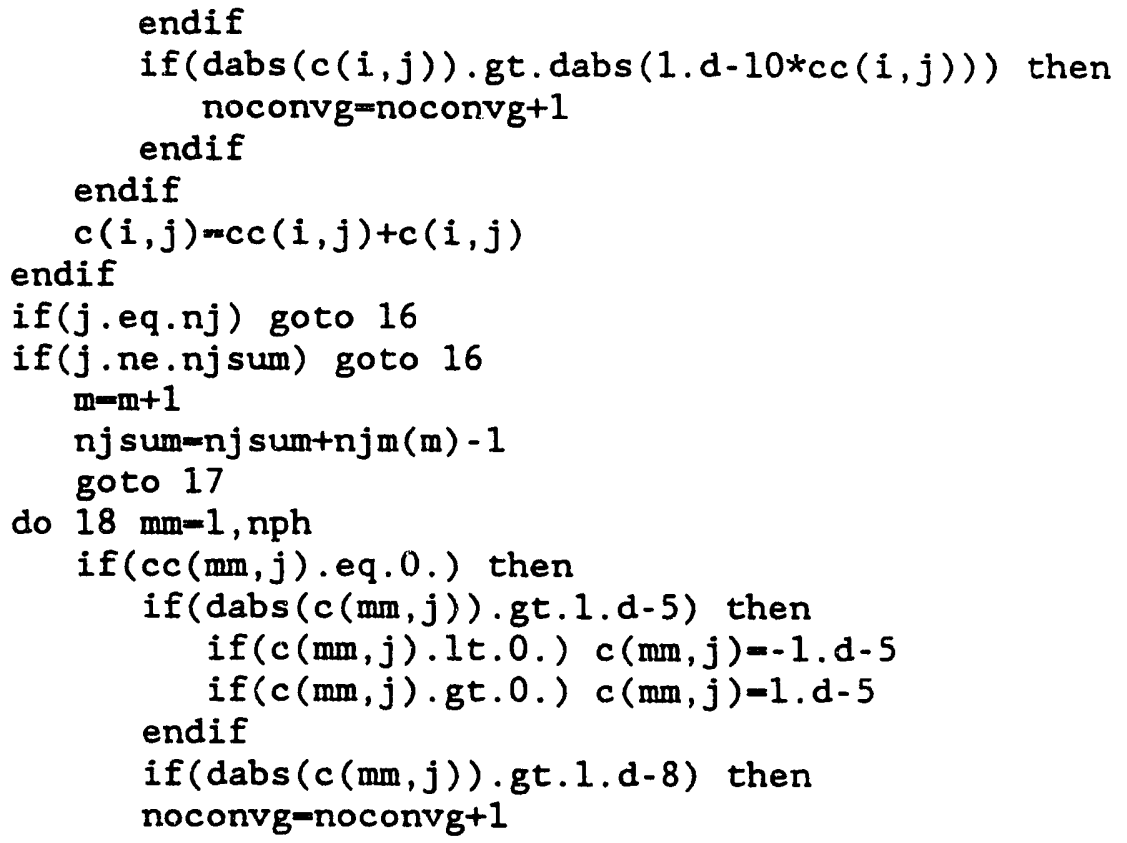




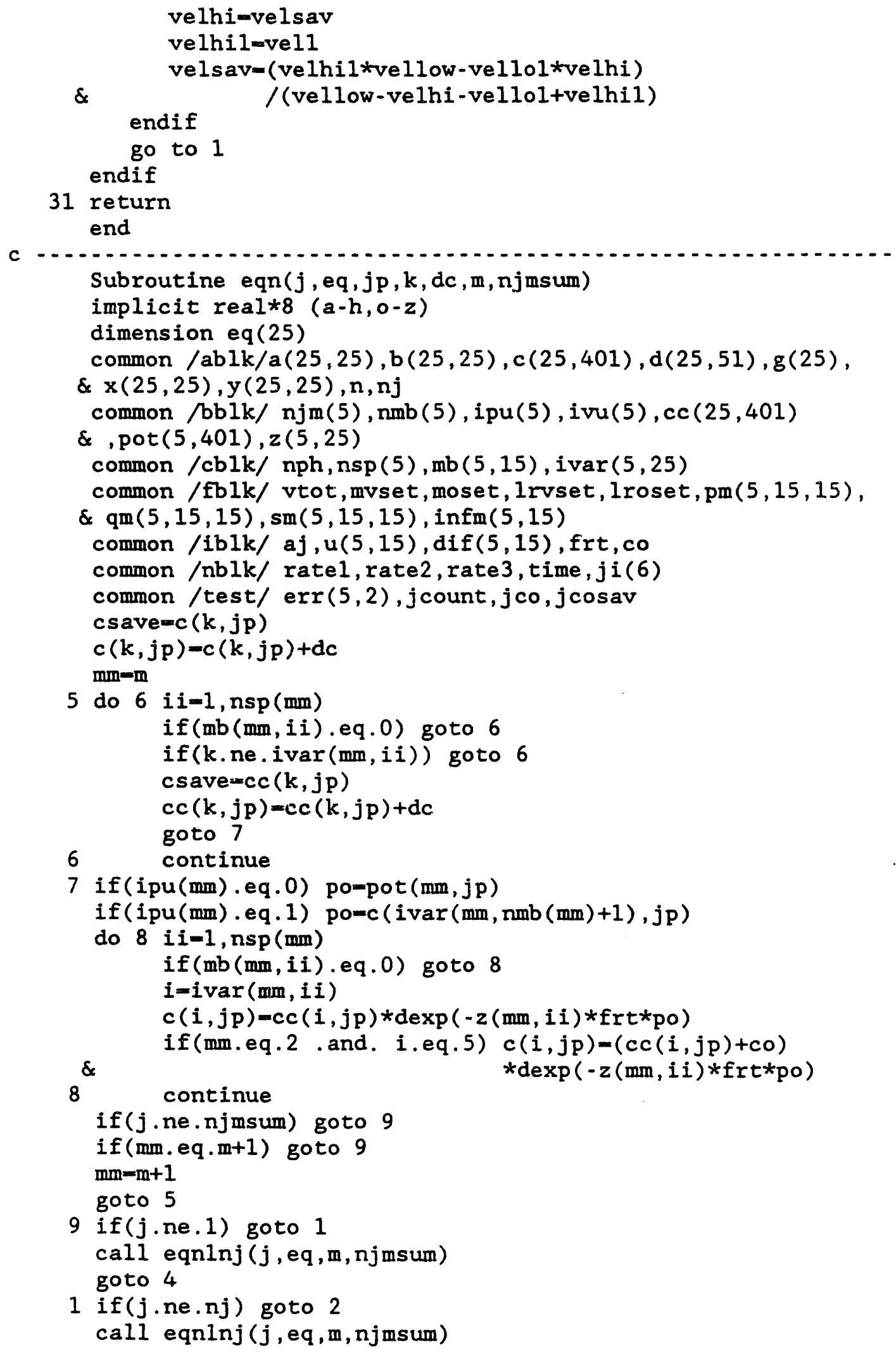


goto 4

2 if (j.ne.njmsum) goto 3

call eqni (j,eq,m, njmsum)

goto 4

3 call eqnj (j,eq, m, njmsum)

4 continue

$c(k, j p)=c s$ ave

$\mathrm{mm}=\mathrm{m}$

10 do $11 \mathrm{ii}=1, \mathrm{nsp}(\mathrm{mm})$

if $(\mathrm{mb}(\mathrm{mm}, \mathrm{ii})$.eq. 0$)$ goto 11

if (k.ne.ivar (mm,ii)) goto 11

$c c(k, j p)=c s a v e$

goto 12

11 continue

12 if (ipu(mm).eq.0) po=pot(mm,jp)

if (ipu (mm).eq.1) po-c(ivar (mm, $\mathrm{nmb}(\mathrm{mm})+1), j p)$

do 13 i $i=1, n s p(\mathrm{~mm})$

if $(\mathrm{mb}(\mathrm{mm}, \mathrm{ii})$.eq. 0$)$ goto 13

$i=i \operatorname{var}(\mathrm{mm}, i \mathrm{i})$

$c(i, j p)=c c(i, j p) * \operatorname{dexp}(-z(m m, i i) * f r t * p o)$

$\&$

if $(\mathrm{mm}$. eq. 2 . and. $i . e q .5) \quad c(i, j p)=(c c(i, j p)+c o)$

13 continue $\star \operatorname{dexp}(-z(\mathrm{~mm}, \mathrm{i} i) * f r t * p o)$

if ( $j$.ne.njmsum) goto 14

if $(\mathrm{mm} . \mathrm{eq} . \mathrm{m}+1)$ goto 14

$m m=m+1$

goto 10

14 return

end

c

Subroutine eqnlnj ( $j, e q, m, n j m s u m)$

implicit real $* 8(a-h, o-z)$

dimension eq (25), ic (25), ih(25)

common /ablk/a $(25,25), \mathrm{b}(25,25), \mathrm{c}(25,401), \mathrm{d}(25,51), \mathrm{g}(25)$,

$\& x(25,25), y(25,25), n, n j t$

common /bblk/ nj(5), nmb(5), ipu(5), ivu(5), cc $(25,401)$

$\&, \operatorname{pot}(5,401), z(5,25)$

common /cblk/ nph, nsp(5), mb $(5,15)$, ivar $(5,25)$

common /fblk/ vtot, mvset, moset, lrvset, 1roset, $\mathrm{pm}(5,15,15)$,

\& $\mathrm{qm}(5,15,15), \operatorname{sm}(5,15,15), \operatorname{infm}(5,15)$

common /hblk/ mvi, $\operatorname{nhmr}(5), \operatorname{cin}(5,15)$, equilm $(5,15)$,

\& $v(5,401), v 1(5,401)$

common /mblk/ $2 \operatorname{mn}(25,25), z \operatorname{mv}(25,25)$, ie $(25), p(25,25)$,

c

$\& q(25,25), s(25,25), \operatorname{dum}(5,2,25)$

c Boundary condition at the far left and right.

c

do $1 \mathrm{ml}=1$, $\mathrm{nph}$

do 1 i=1, n

$\operatorname{dum}(m 1,1, i)=1$.

$\operatorname{ih}(i)=0$ 


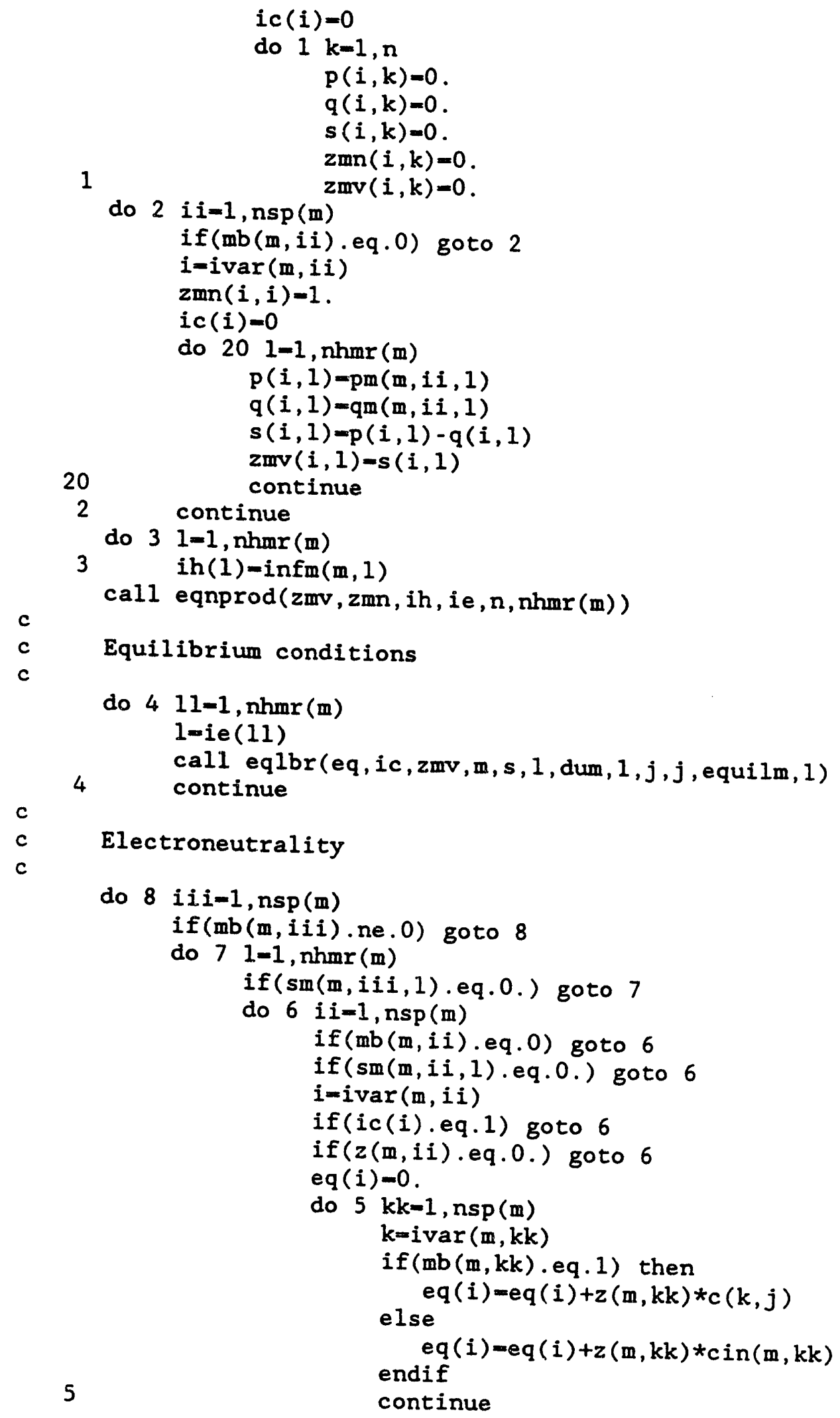




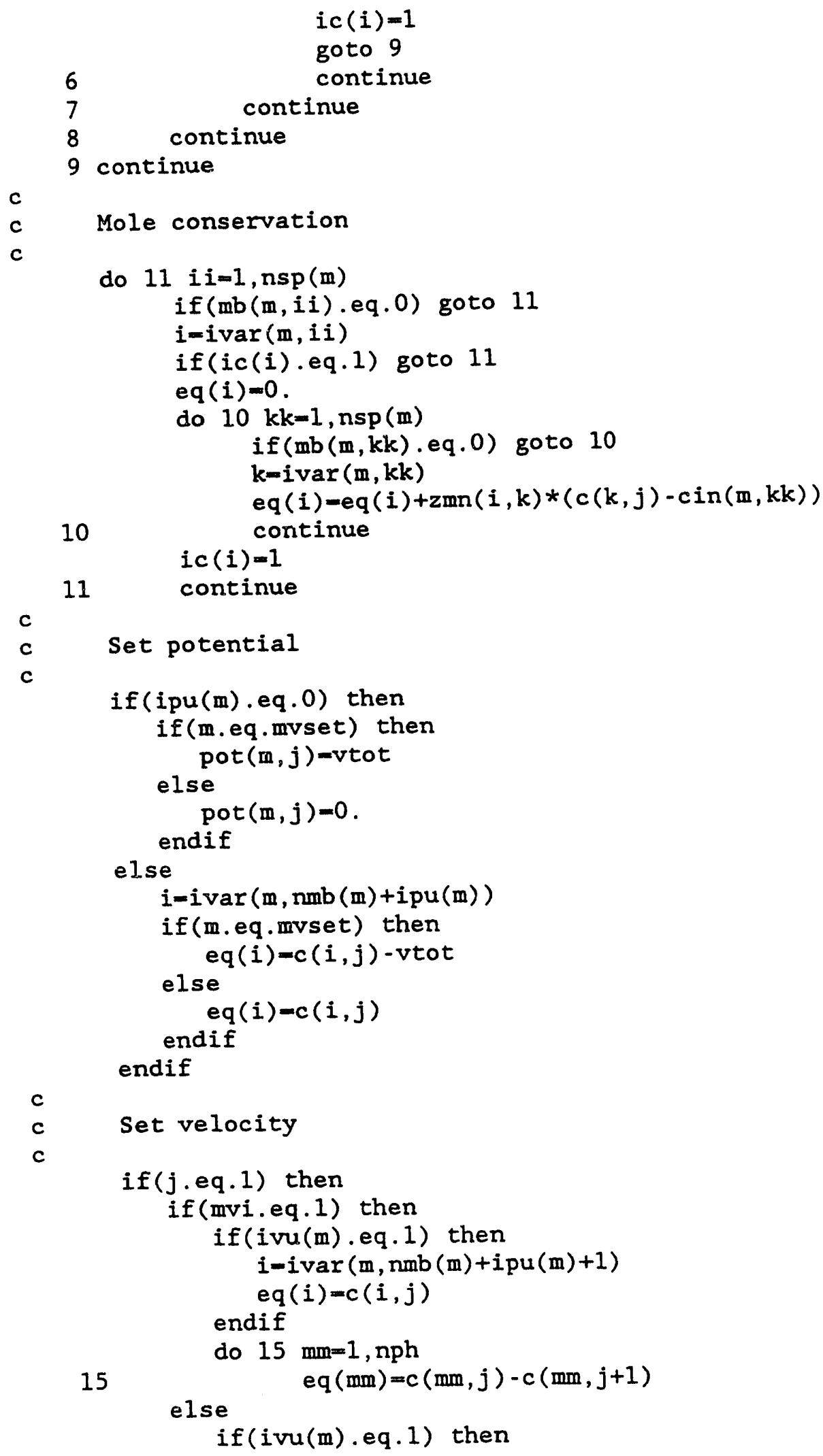




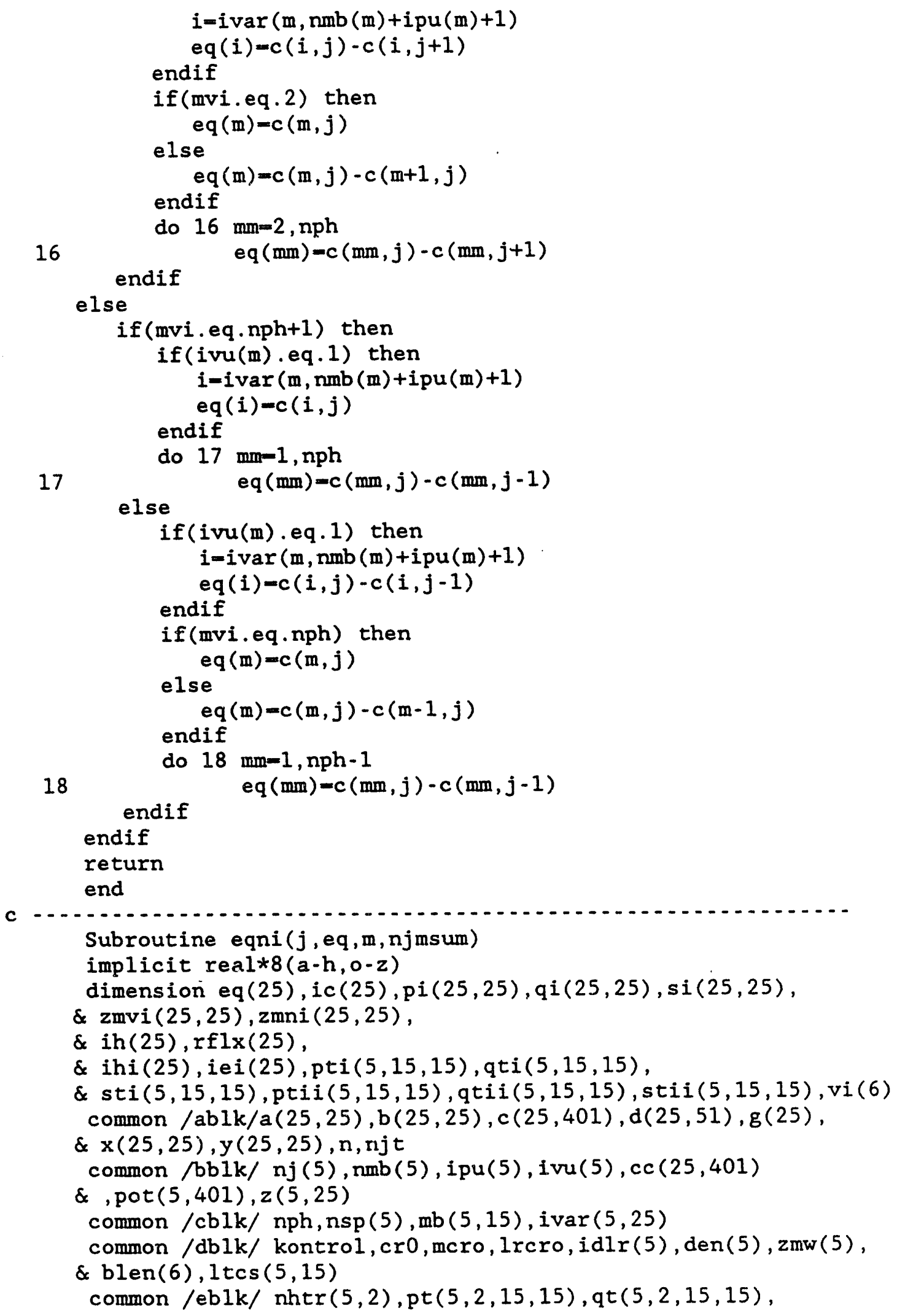


$\& \operatorname{st}(5,2,15,15), \operatorname{inft}(6,15), \operatorname{rkba}(6,15)$, equilt $(6,15)$,

$\&$ be $(6,15), z k s(5,2,25), \operatorname{curl}(6), \operatorname{curr}(6)$

common /fblk/ vtot, mvset, moset, lrvset, 1roset, $\operatorname{pm}(5,15,15)$,

$\& \operatorname{qm}(5,15,15), \operatorname{sm}(5,15,15), \operatorname{infm}(5,15)$

common /gblk/ zlen(5), ninfm(5), $\operatorname{rkbv}(5,15), \mathrm{h}(5,401), \operatorname{perm}(5), f$

$\&, \operatorname{ccsav}(25,401) \operatorname{sav}(25,401)$, yy $(5,401)$

common /hblk/ miri, $\operatorname{nhmr}(5), \operatorname{cin}(5,15)$, equilm $(5,15)$,

$\& \mathrm{v}(5,401), \mathrm{vl}(5,401)$

common /iblk/ aj, $u(5,15)$, dif $(5,15)$, frt, co

common /mblk/ $z \operatorname{mn}(25,25), z \operatorname{mv}(25,25)$, ie $(25), \mathrm{p}(25,25)$,

$\& q(25,25), s(25,25), \operatorname{dum}(5,2,25)$

common /test/ err $(5,2), j$ count, jco,jcosav

common/rate/velsav, vel, vell, $\operatorname{rrxn}(25), \operatorname{cur}(401), \operatorname{cursav}(401), j$ time

$\&, f 1 x s(401,15), f l x s a v(401,15), \operatorname{chd}(401), \operatorname{chdsav}(401), \operatorname{currsav}(6)$

Equations at an interior boundary.

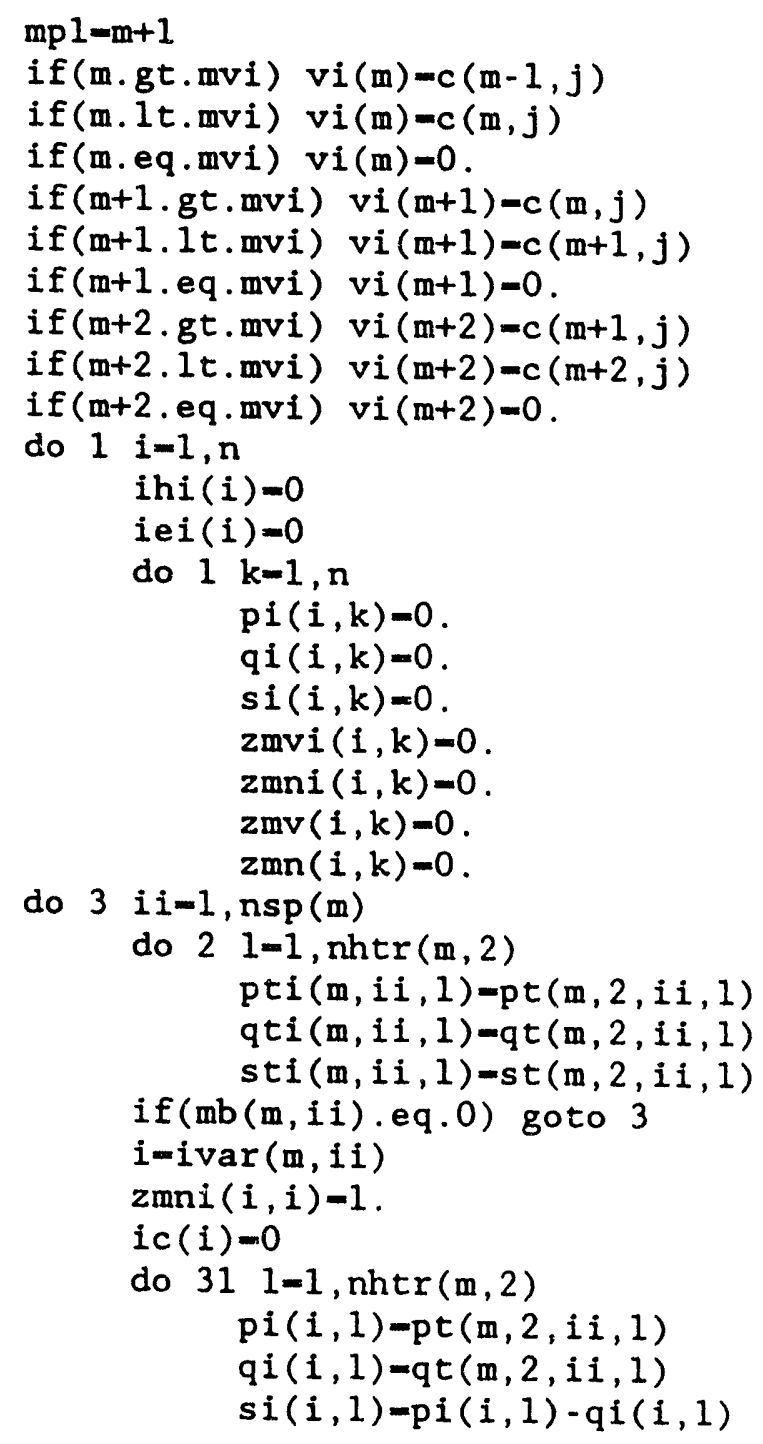




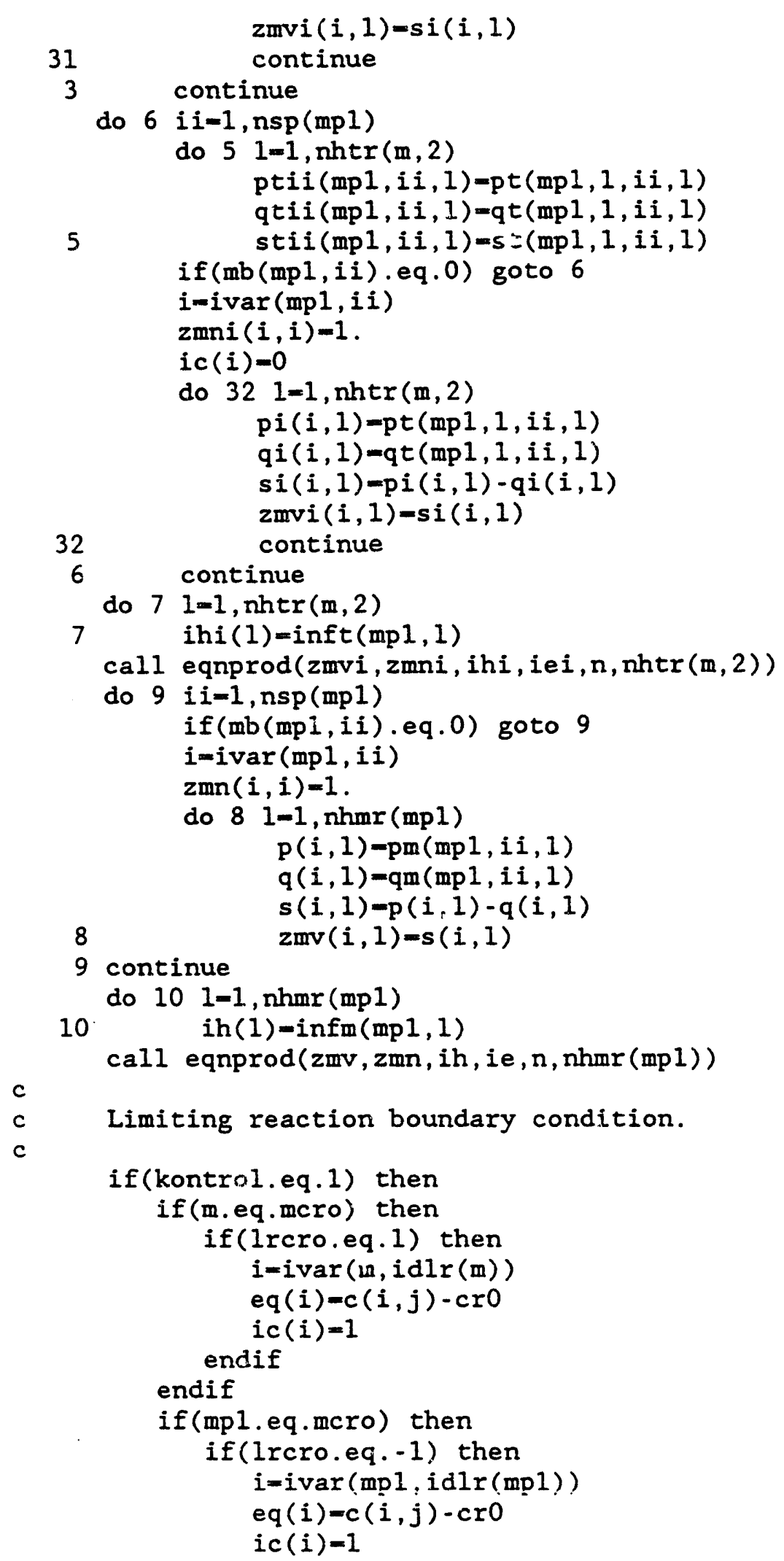




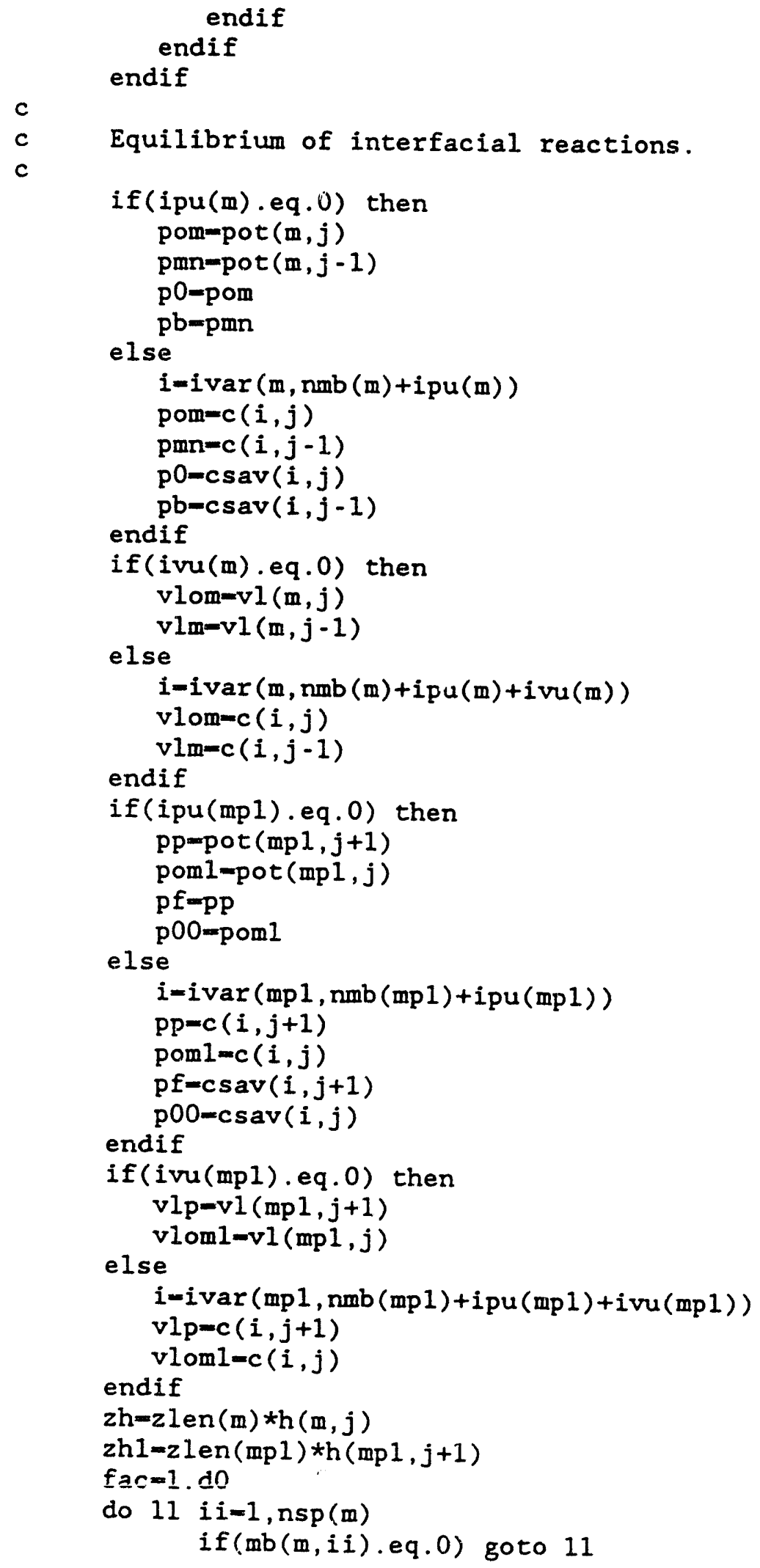




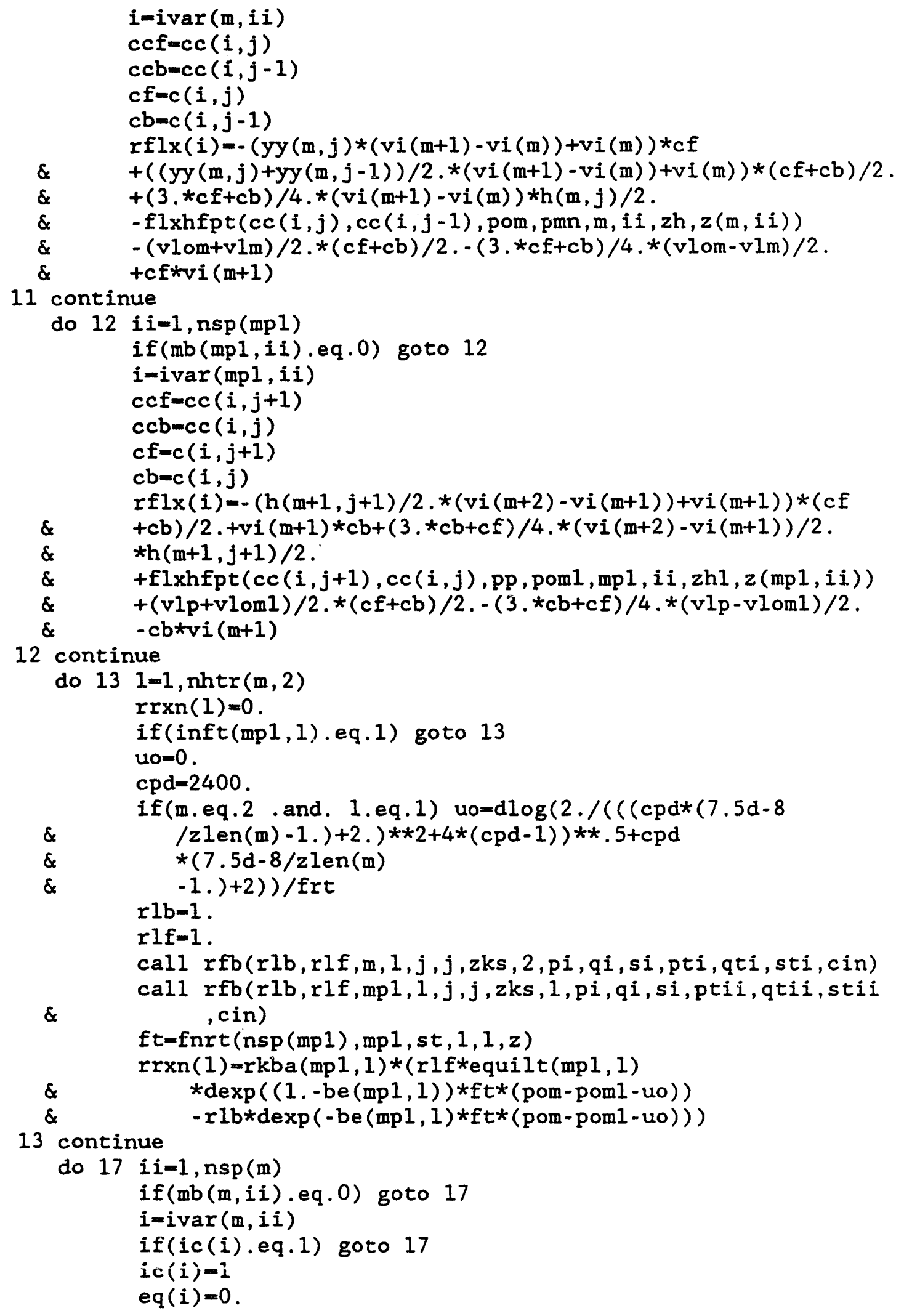


14

continue

infrxn $=0$

do 14 1=1, nhtr (m,2)

if (zmvi( $i, 1)$.eq. 0$)$ goto 14

if $(\operatorname{inft}(\operatorname{mpl}, 1)$.eq.1) then

infrxn-1

goto 15

else

$e q(i)=e q(i)+z m v i(i, 1) * \operatorname{rrxn}(1)$

endif

do $16 \mathrm{kk}=1, \mathrm{nsp}(\mathrm{m})$

if (mb(m,kk).eq.0) goto 16

$k=i \operatorname{var}(m, k k)$

if $(z \operatorname{mni}(i, k)$.eq. 0$)$ goto 16

if (infrxn.eq.1) then

$\operatorname{rrxn}(1)=\operatorname{rrxn}(1)-z \operatorname{mni}(i, k) / z \operatorname{mvi}(i, 1) * \operatorname{rflx}(k)$ else endif

$e q(i)=e q(i)+z m n i(i, k) * r f l x(k)$

16

continue

do $40 \mathrm{kk}=1, \mathrm{nsp}(\mathrm{mp} 1)$

if (mb(mpl, kk).eq.0) goto 40

$\mathrm{k}$-ivar (mpl,kk)

if (zmni $(i, k)$.eq. 0$)$ goto 40

if (infrxn.eq. 1) then

$\operatorname{rrxn}(1)=\operatorname{rrxn}(1)-z \operatorname{mn} i(i, k) / z \operatorname{mv} i(i, 1) * r f l x(k)$ else endif

$e q(i)=e q(i)+z m n i(i, k) * r f l x(k)$

40

continue

if(infrxn.eq.0) goto 17

eq $(i)=0$.

call smslnc (eq (i), m, si, $1, z k s, 2, j, j)$

call smslnc (eq (i) , mp $1, s i, 1, z k s, 1, j, j)$

$f t=f \operatorname{nrt}(\operatorname{nsp}(m), m, s t, 2,1, z)$

uo $=0$.

cpd $=2400$.

if (m.eq. 2 and. 1 .eq. 1$)$ uom $\operatorname{dlog}(2 . /(((\operatorname{cpd} *(7.5 \mathrm{~d}-8$

\& $\quad / z \operatorname{len}(\mathrm{m})-1)+2.) * * 2+4 *(\mathrm{cpd}-1)) * * .5+\mathrm{cpd}$

$\& \quad *(7.5 d-8 / 2 \operatorname{len}(m)$

\& $\quad-1)+2).) /$ frt

17 continue

$e q(i)=e q(i)+d \log ($ equilt $(m p 1,1))+f t *(p o m-p o m l-u o)$

do $23 i i=1, n s p(m p 1)$

if (mb(mpl,ii).eq. 0$)$ goto 23

$i=i \operatorname{var}(\operatorname{mp} 1, i i)$

if (ic(i).eq. 1 ) goto 23

ic $(i)=1$

eq $(i)=0$.

inf $r \times n=0$

do $201=1, \operatorname{nhtr}(m, 2)$ 


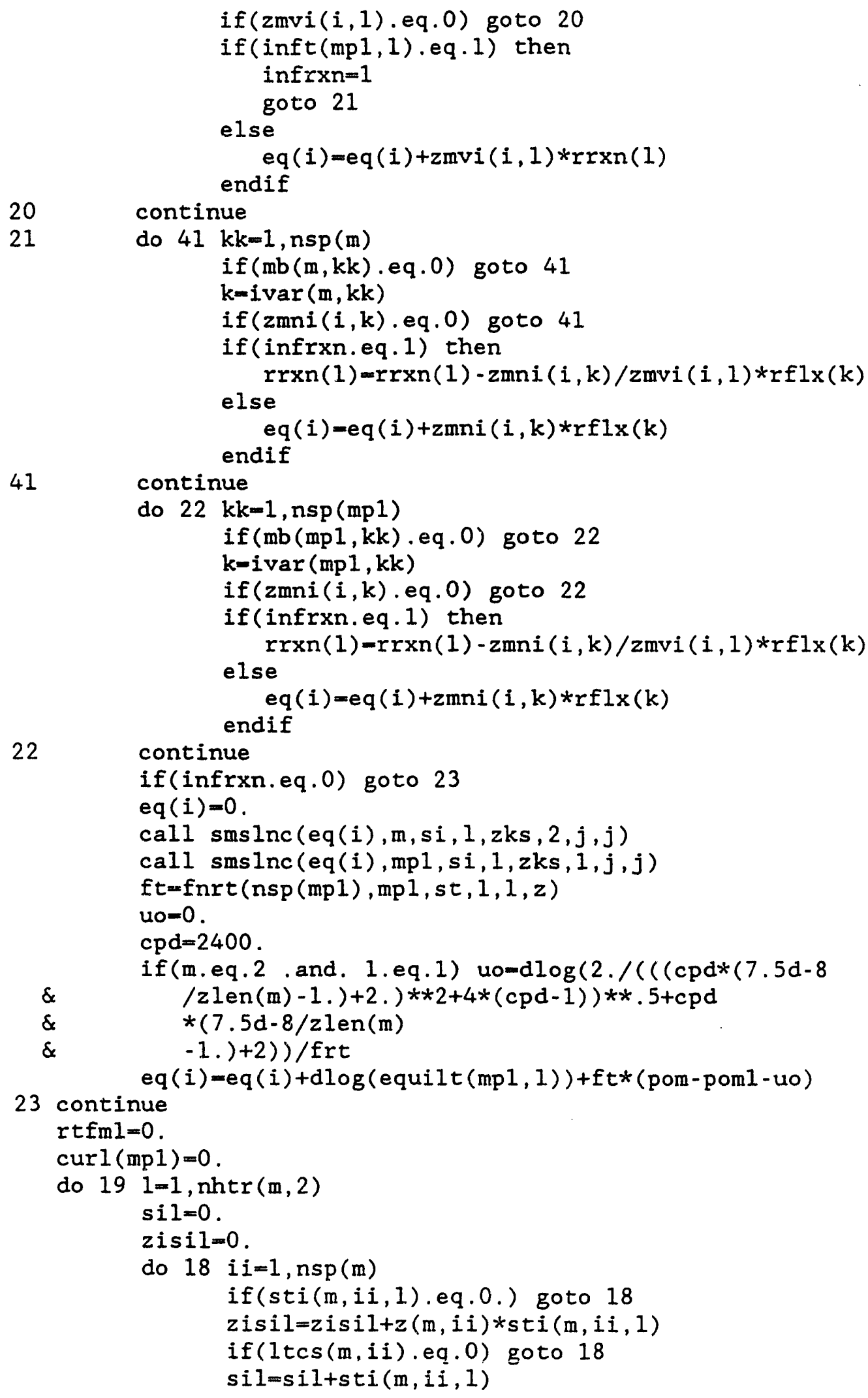




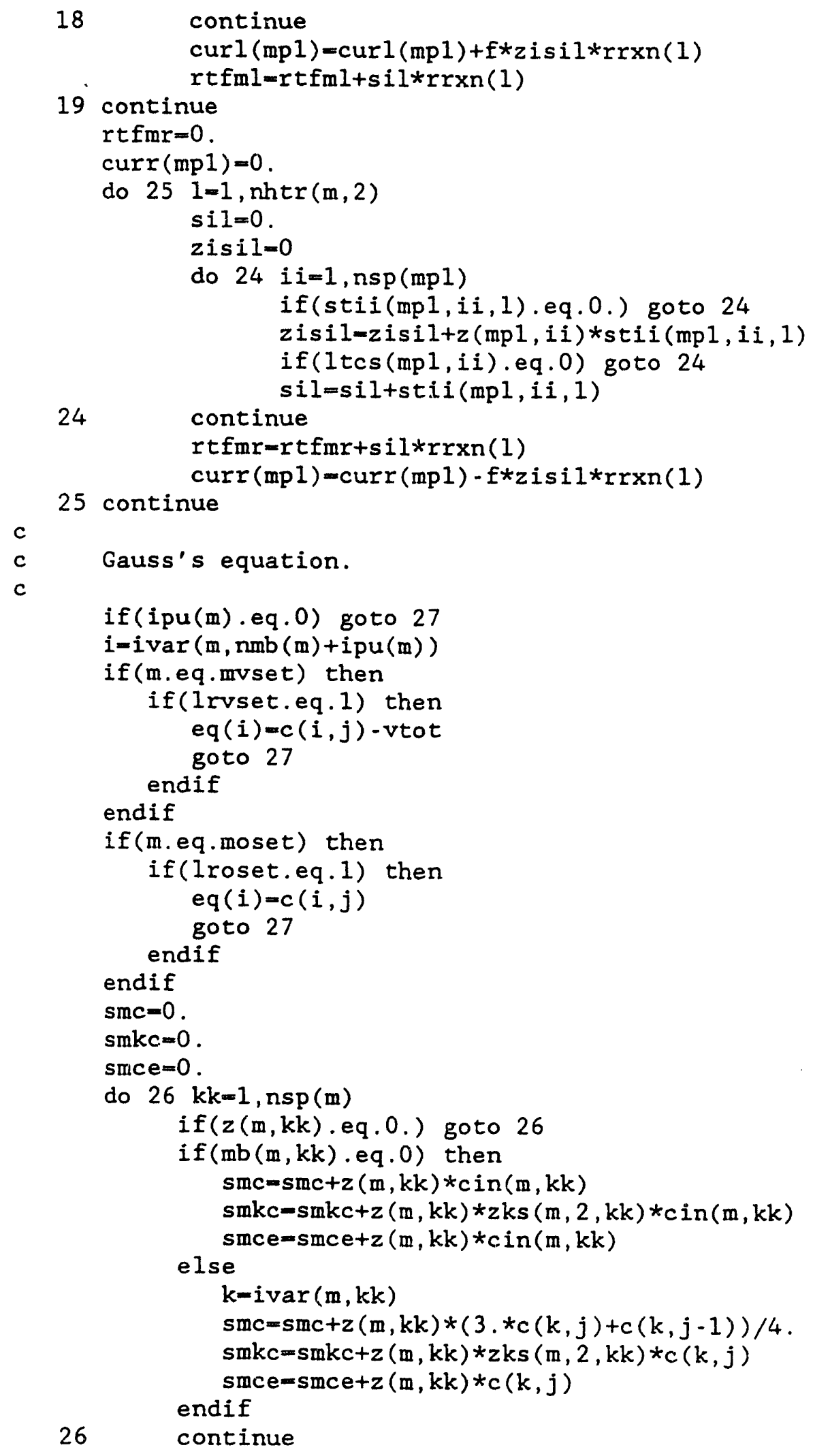




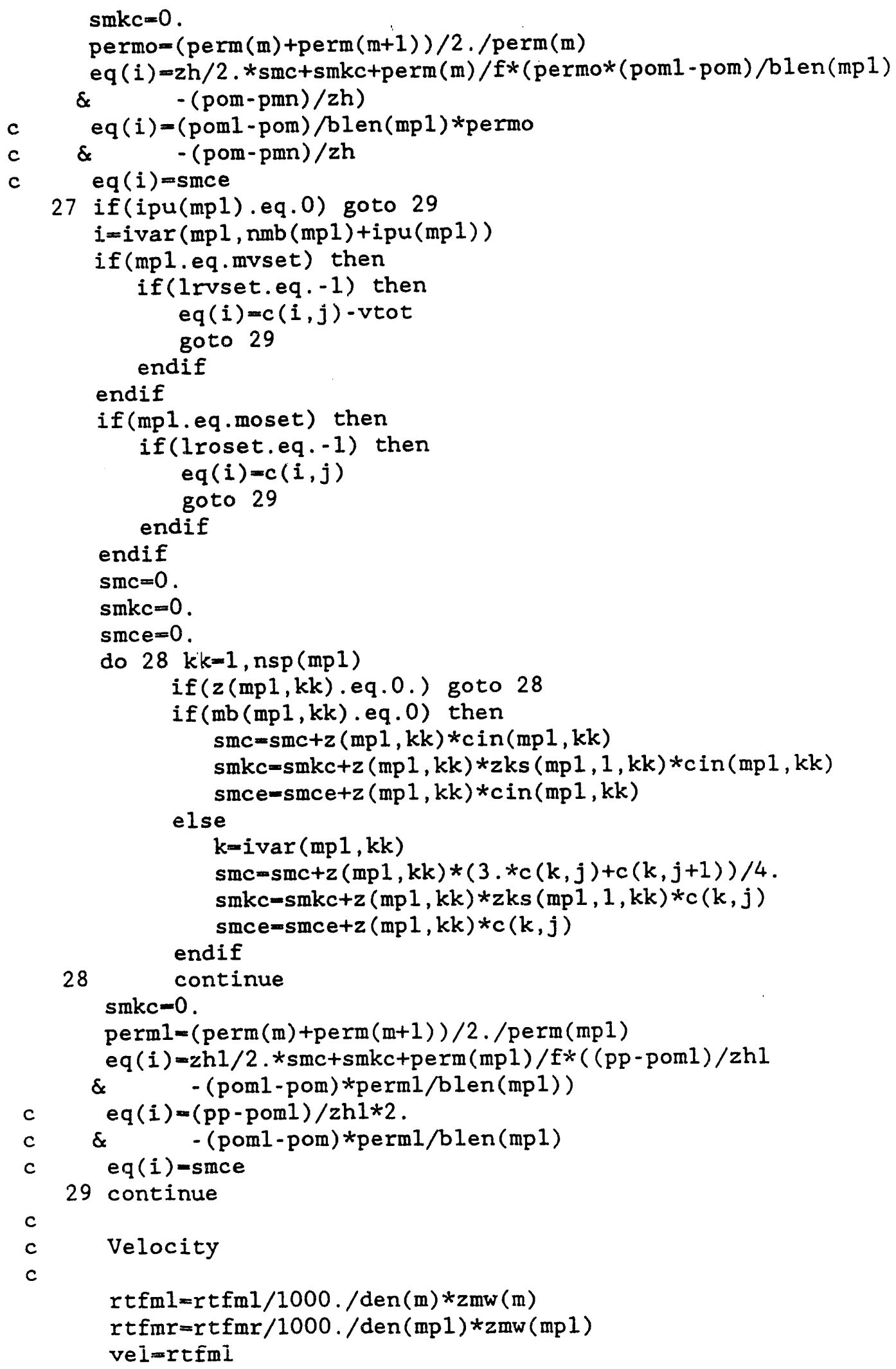




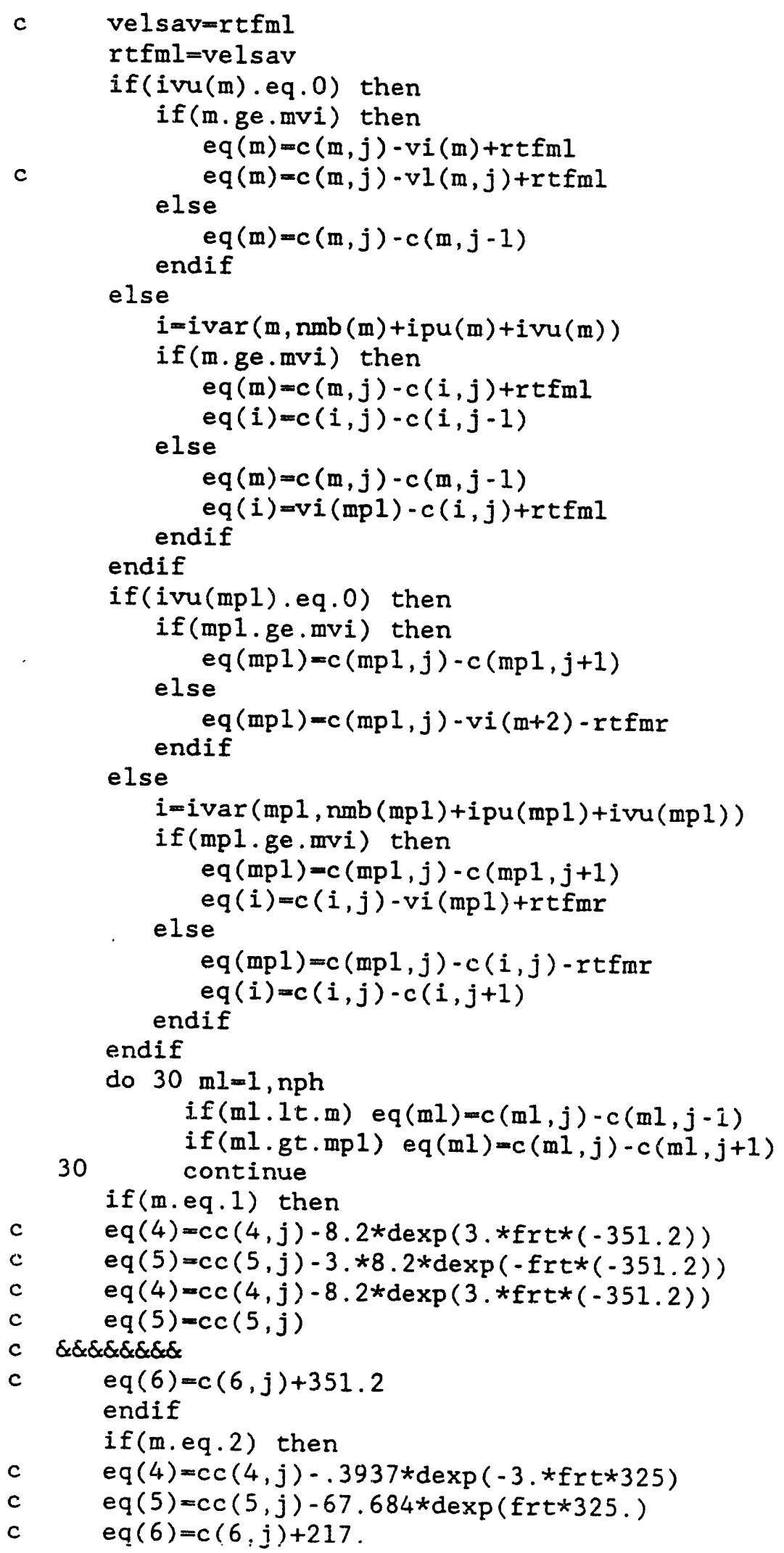




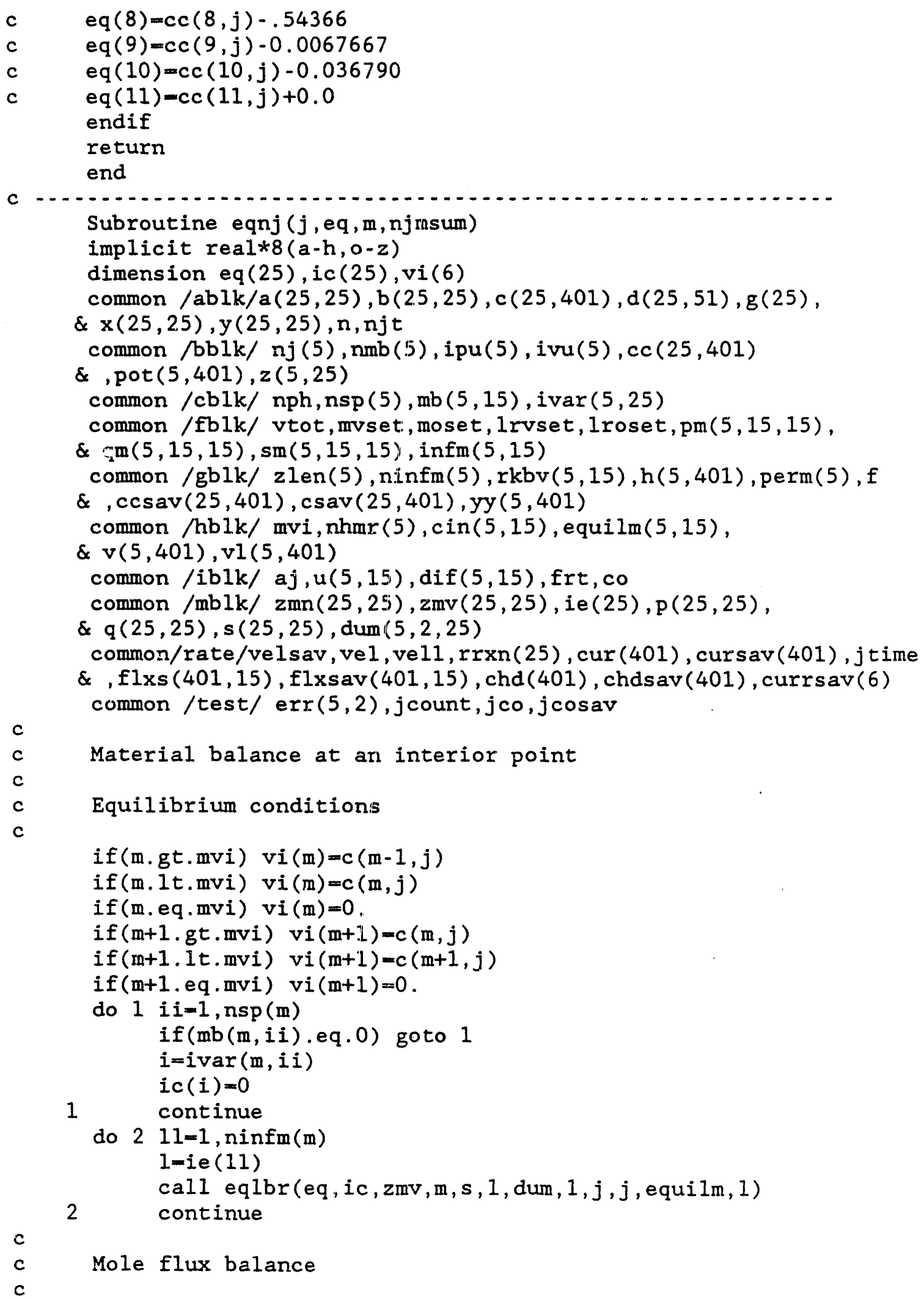




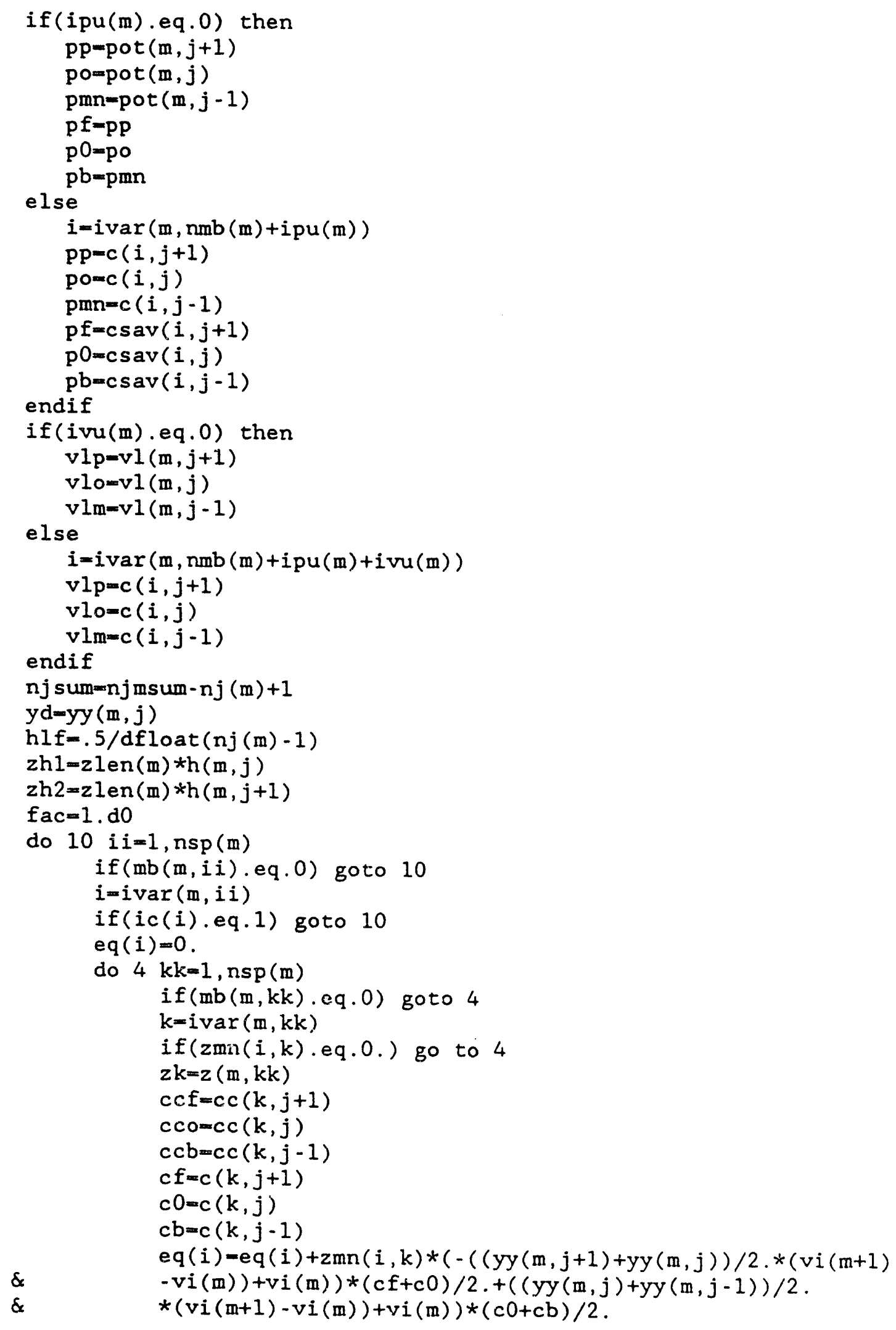




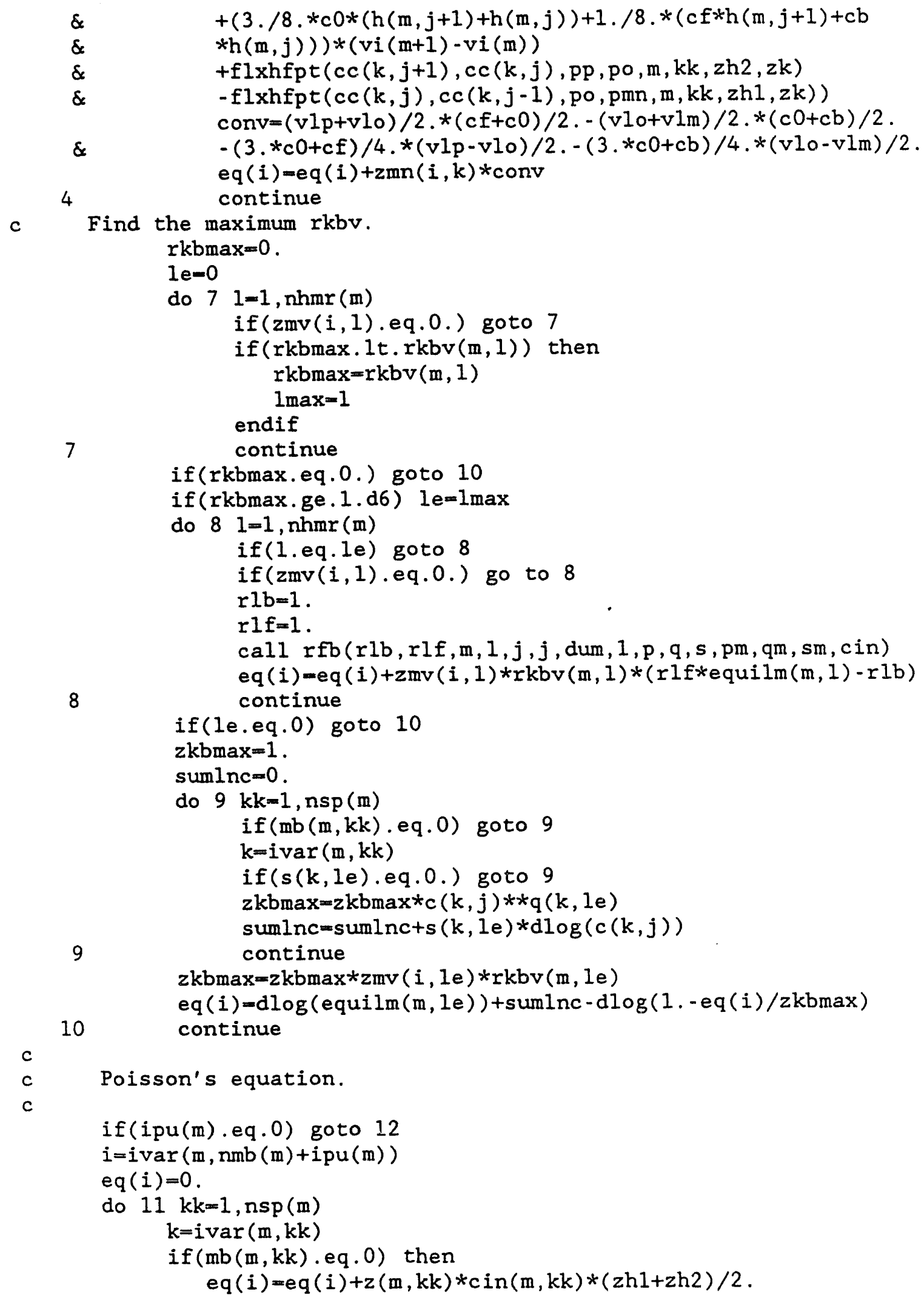




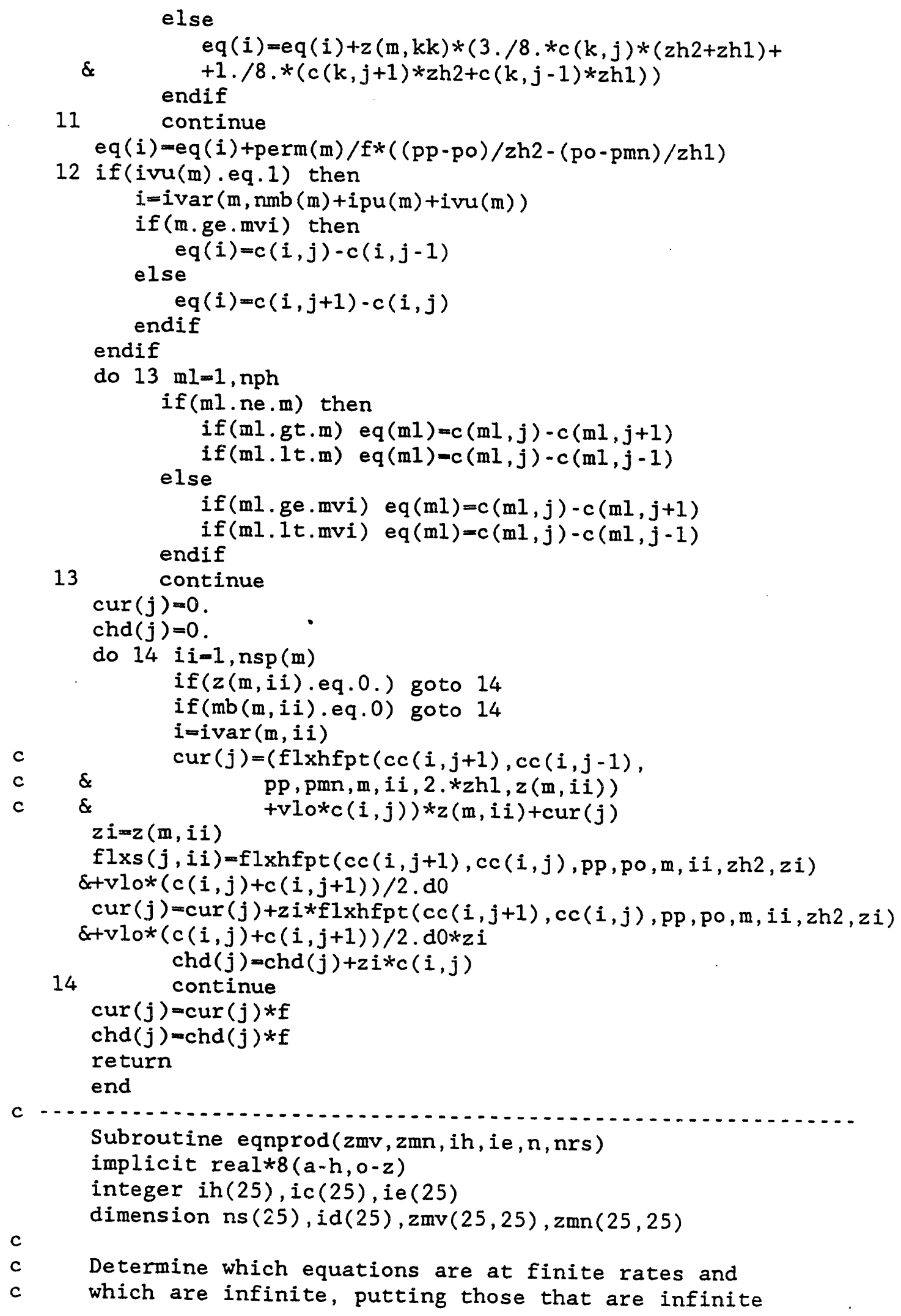


c at the front of an identifying vector, ie, and the

c others at the back.

c This will allow me to set up the equations such that

c the equilibrium equations are defined first.

c

$$
\text { do } 1 \quad 1=1 \text {, nrs }
$$

1 ie $(1)=0$

$j=0$

$k=-1$

do $31=1$, nrs

if (ih(1).ne.1) goto 2

$j=j+1$

ie $(j)=1$

goto 3

2

$\mathrm{k}=\mathrm{k}+1$

3 continue

ie $(\mathrm{nrs}-\mathrm{k})=1$

$c$

Define an identifying vector, ic, so that I know which equations

c have been used as the pivot and that they are not repeated.

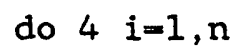

4 ic $(i)=0$

do $191=1$, nrs ie $(1)=1$ $\mathrm{m}=\mathrm{ie}(1)$

c Define a vector, ns, which tells me how many different reactions

c each species is involved in. do $7 i=1, n$ ns $(i)=0$

do $711=1$, nrs if $(z \operatorname{mv}(i, 11) \cdot$ ne.0. $)$ ns $(i)=n s(i)+1$

7 continue

c Determine the pivot point by picking a species which hasn't

c been chosen before $(i c(*)=0)$, and is involved in the fewest

c reactions (but greater than zero.) $\mathrm{j}=0$

do $10 i=1, n$

$8 \quad$ if $(\operatorname{zmv}(i, m)) 9,10,9$

$9 \quad j=j+1$

10 continue

$i d(j)=i$

if (j.eq.0) goto 19

nsmin=ns $(i d(1))$

i $z=i d(1)$

do $11 \mathrm{k}=2, \mathrm{j}$

if (nsmin.gt.ns(id(k))) then $n s m i n=n s(i d(k))$

endif

$i z=i d(k)$

11 continue

c $(i z, m)$ is the pivot point. 


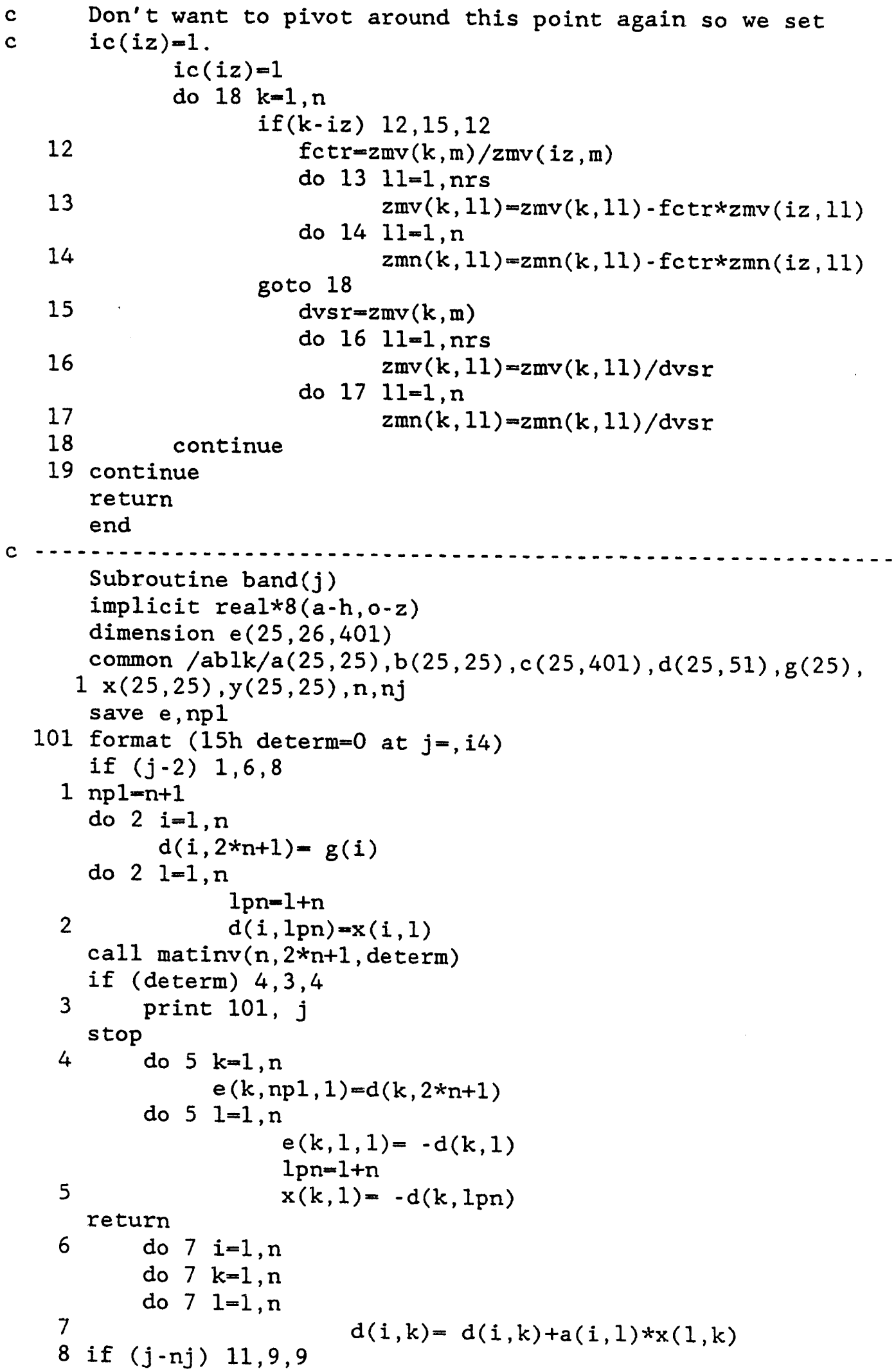




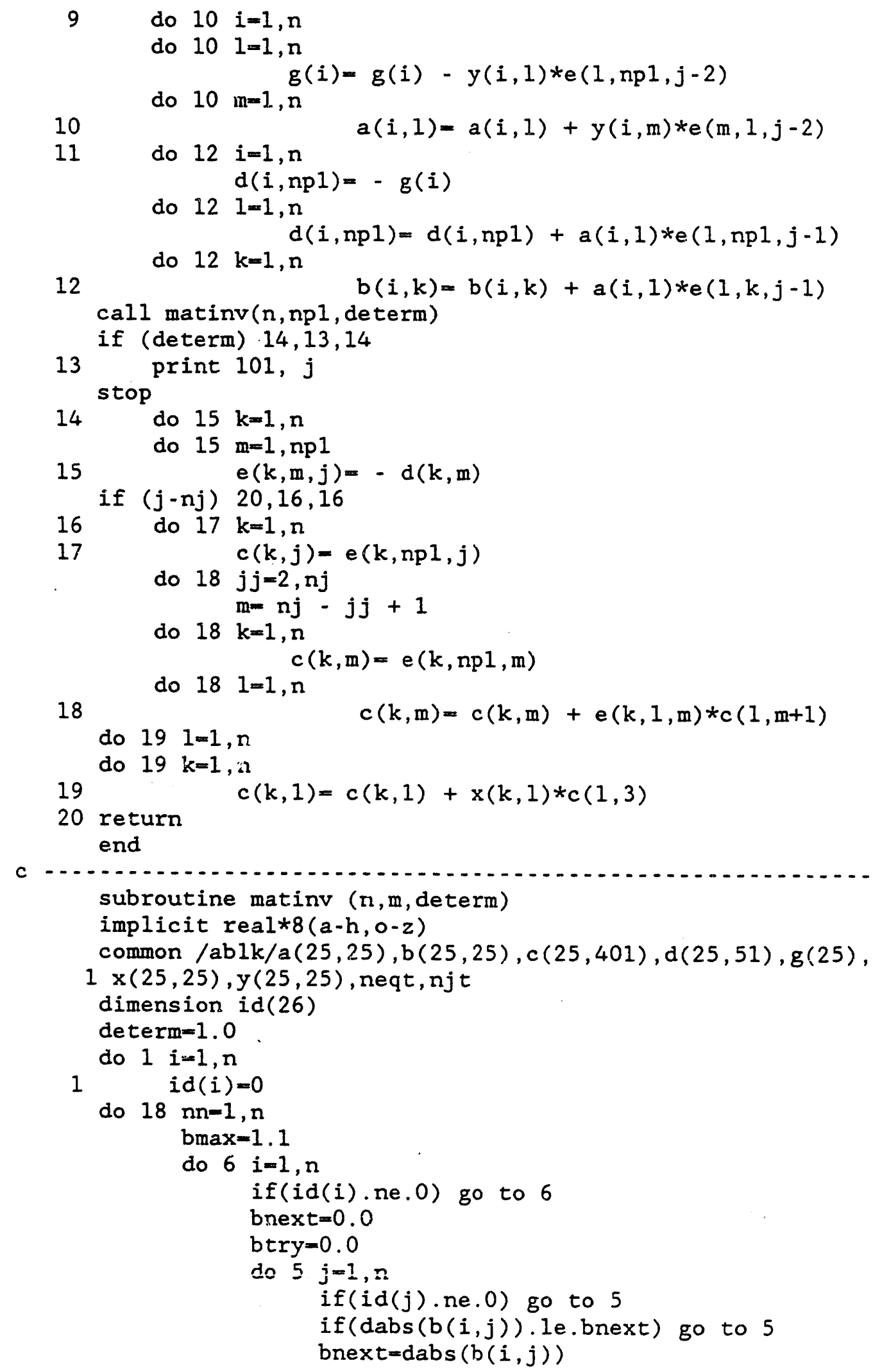




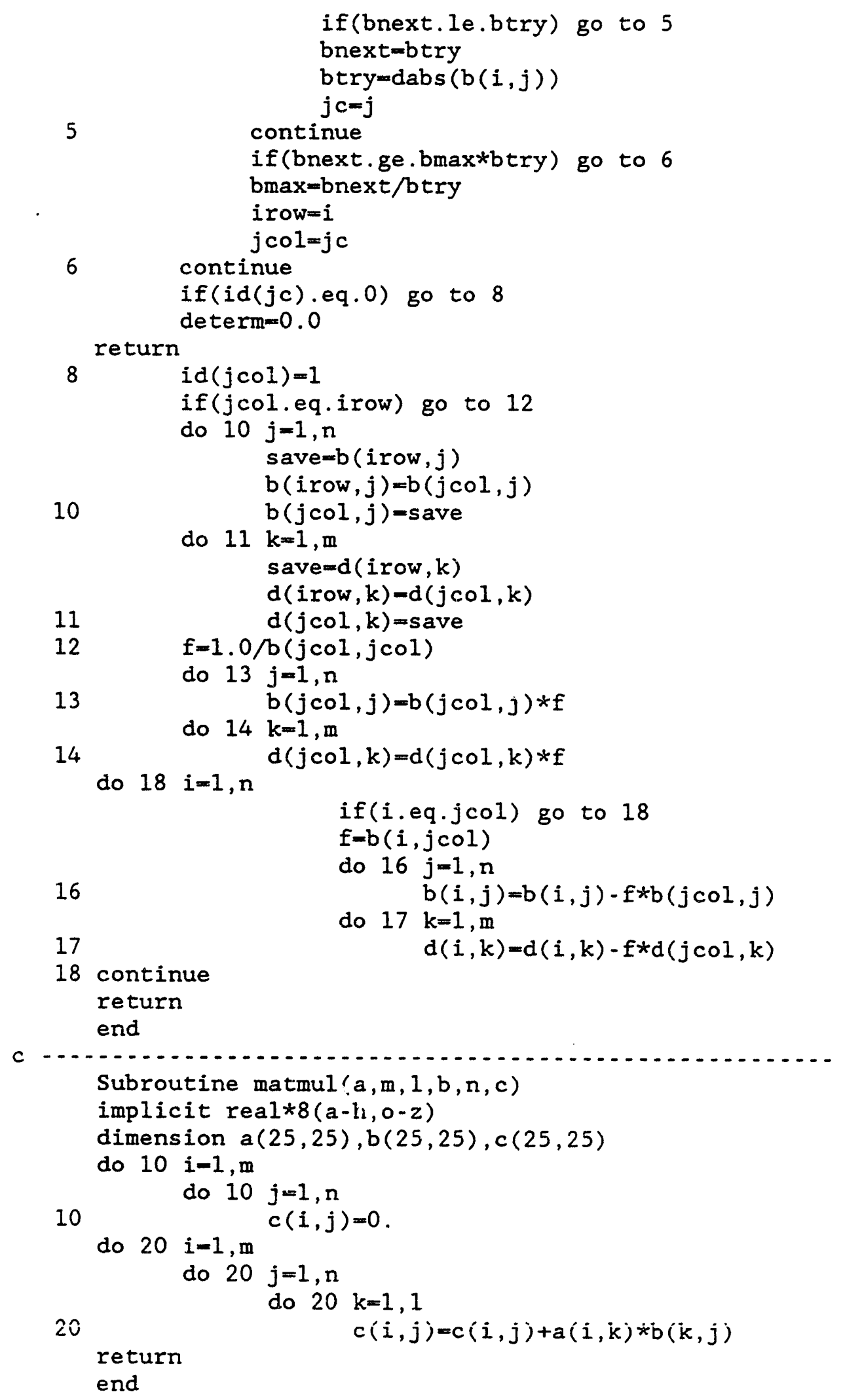


c

Subroutine eqlbr(eq,ic, zmv, $m, s, 1, z k s, 1 r, j, j p$, equilm, 11)

implicit real $* 8(a-h, o-z)$

dimension eq (25), ic (25), zmv $(25,25), s(25,25), z \mathrm{ks}(5,2,25)$,

$\&$ equilm $(5,25)$

common /ablk/a $(25,25), b(25,25), c(25,401), d(25,51), g(25)$,

$\& x(25,25), y(25,25), n, n j t$

common /cblk/ nph, nsp (5), mb $(5,15), i \operatorname{var}(5,25)$

do $1 i i=1$, nsp(m)

if $(\mathrm{mb}(\mathrm{m}, \mathrm{i} i)$.eq. 0$)$ goto 1

$i=i \operatorname{var}(m, i i)$

if (ic(i).eq.1) goto 1

if $(\operatorname{zmv}(i, 1) . e q .0$.$) goto 1$

eq $(i)=0$.

call smslnc (eq (i) , m, s, 1, zks, lr,j,jp)

$e q(i)=e q(i)+d \log ($ equilm $(m, 11))$

ic $(i)=1$

goto 2

1 continue

2 return

end

c

Subroutine smslnc(sum, m, s, 1, zks, $1 r, j, j p)$

implicit real $* 8(a-h, 0-z)$

dimension $s(25,25), z k s(5,2,15)$

common /ablk/a $(25,25), b(25,25), c(25,401), d(25,51), g(25)$,

$\& \mathrm{x}(25,25), \mathrm{y}(25,25), \mathrm{n}, \mathrm{nj}$

common /bblk/ njm(5), nmb(5), ipu(5), iva(5), cc $(25,401)$

$\&, \operatorname{pot}(5,401), z(5,25)$

common /iblk/ aj,u(5,15), dif $(5,15)$, frt, co

common /cblk/ nph, nsp (5), mb $(5,15)$, ivar $(5,25)$

if (ipu(m).eq.0) pl=pot(m,j)

if (ipu(m).eq.0) p2=pot(m,jp)

if (ipu(m).eq.1) $\mathrm{p} 1=\mathrm{c}(\mathrm{ivar}(\mathrm{m}, \mathrm{nmb}(\mathrm{m})+1), j)$

if (ipu(m).eq.1) $\mathrm{p} 2=\mathrm{c}(\mathrm{ivar}(\mathrm{m}, \mathrm{nmb}(\mathrm{m})+1), j \mathrm{p})$

do $1 \mathrm{kk}=1, \mathrm{nsp}(\mathrm{m})$

if $(\mathrm{mb}(\mathrm{m}, \mathrm{kk})$.eq. 0$)$ goto 1

$k=i \operatorname{var}(m, k k)$

if $(c(k, j)$. le.0.) goto 1

c $\operatorname{sum}=\operatorname{sum}+s(k, 1) * d \operatorname{dog}(z k s(m, 1 r, k k) *(3 . * c(k, j)+c(k, j p)) / 4$. if (k.eq.5) then if $(3 . * c c(k, j)+c c(k, j p)+4 . * c o .1 e .0$.$) goto 1$ sum $=\operatorname{sum}+s(k, 1) *(d \log (z k s(m, 1 r, k k) *$

$\& \quad(3 . * c c(k, j)+c c(k, j p)) / 4 .+c o)$

$\& \quad-z(m, k k) * f r t *(3 . * p 1+p 2) / 4$.

else

if $(3 . * c c(k, j)+c c(k, j p) .1 e .0$.$) goto 1$

$\& \quad(3 . * c c(k, j)+c c(k, j p)) / 4$.

$\& \quad-z(m, k k) * f r t *(3, * p 1+p 2) / 4$. endif 


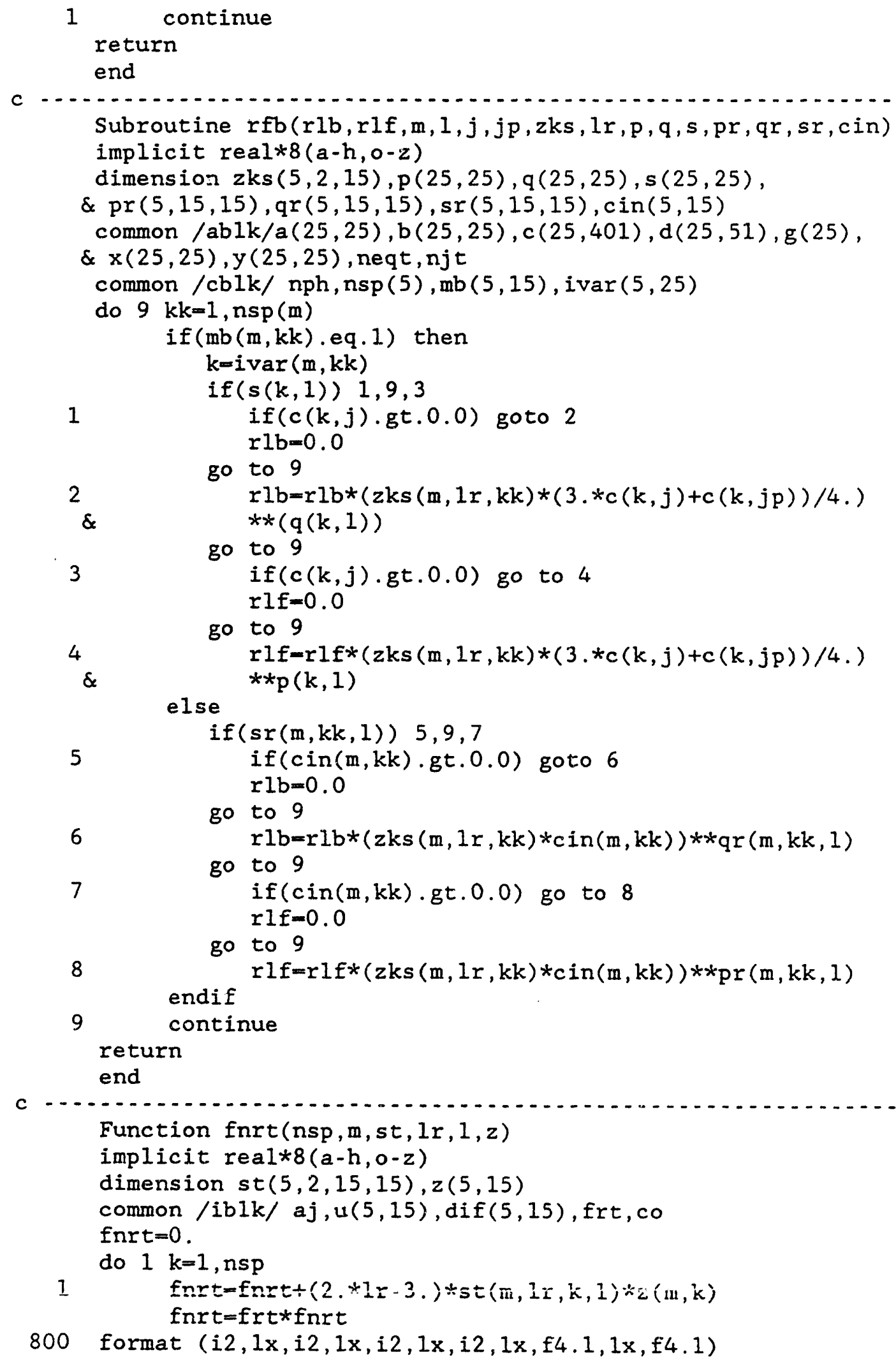




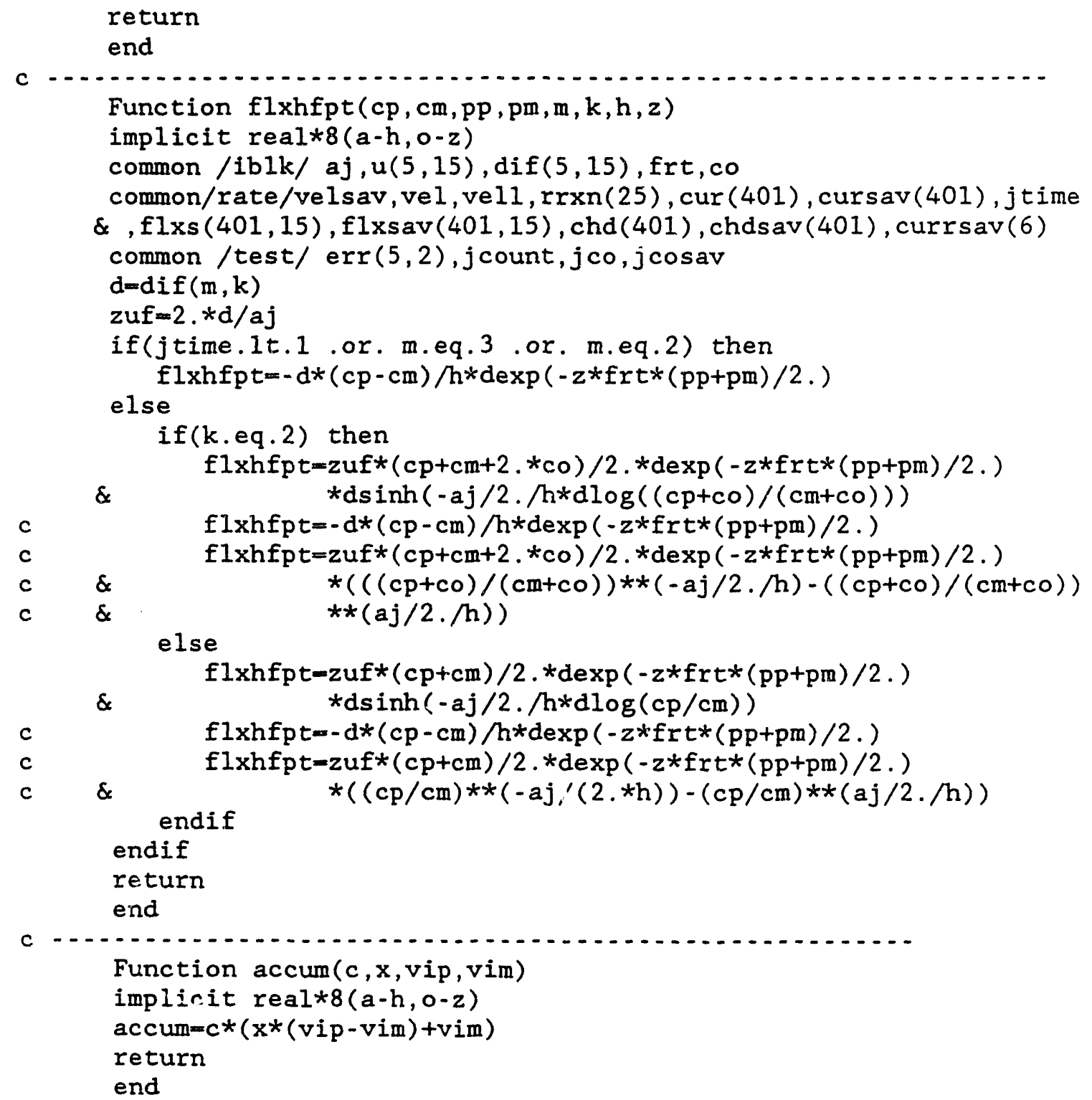


Data File

iren.dat

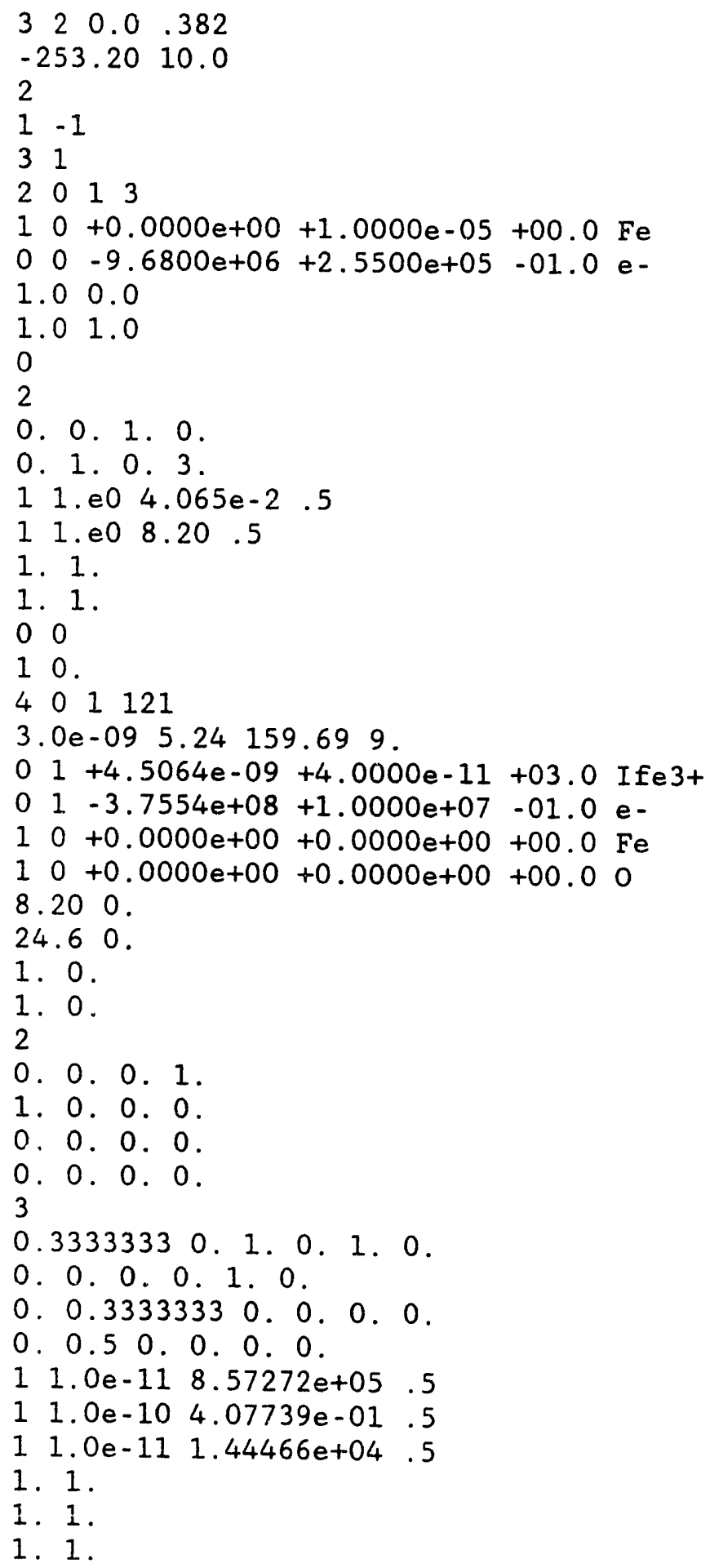


1. 1 .

11

$\begin{array}{lllll}6 & 0 & 4 & 181\end{array}$

3. 1.18 .078 .

$01+5.0110 e+01+1.3340 e+00+01.0 \mathrm{Na}+$

$01+5.4000 \mathrm{e}+01+0.7200 \mathrm{e}+00+02.0 \mathrm{Fe} 2+$

$01+3.7554 \mathrm{e}+01+1.0000 \mathrm{e}+00+01.0 \mathrm{FeOH} 2$

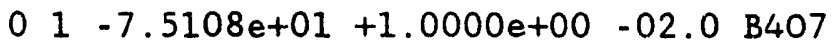

$\begin{array}{lllll}0 & 1 & -3.7554 \mathrm{e}+01+1.0000 \mathrm{e}+00 & -01.0 \mathrm{HB} 407\end{array}$

$00+0.0000 e+00+4.0000 e+00+00.0$ H2O

0.200 .

$1.0 e-110$.

1. $3 e-180$.

0.050000000010 .

0.100 .

1.01 .0

3

0. 0. 0. 0. 0. 0 .

0.0 .0 .0 .0 .1 .

0 . 0.0.1.0. 0 .

1. 0. 2. 0. 0. 0 .

0. 1. 0. 2. 0. 0 .

0.50 .2 . 0.0 .0 .

0

1. 1 .

1. 1 .

1. 1 .

1. 1 .

1. 1 .

1. 1 .

10

$4-1$ 
Chapter 4

The Oxidation of Thiosulfate on Copper for Electroless Deposition

\section{Foreword}

An investigation of sodium thiosulfate as a possible reducing agent for electroless copper deposition at $25^{\circ} \mathrm{C}$ using a copper rotating disk electrode was carried out. The polarization curves of solutions containing copper sulfate and EDTA were obtained, as were the polarization curves of solutions of thiosulfate and EDTA. It was determined that the optimum potential range for copper deposition in the presence of EDTA is $-1.3 \mathrm{to}-1.1 \mathrm{~V}$ relative to a $\mathrm{Hg} / \mathrm{HgO} / 1 \mathrm{M} \mathrm{NaOH}$ reference electrode. It was also found that the oxidation currents of solutions of thiosulfate with EDTA are roughly linearly dependent on the amount of thiosulfate present and are dependent on the concentration of EDTA to the one-fifth power. Ultimately, it appears that thiosulfate form: a stable intermediate with cuprous ions, thereby precluding electroless deposition of cupric ions.

\section{Ini roduction}

Thiosulfate is investigated as a possible reducing agent for the electroless deposition of copper by means of polarization data derived with a copper rotating disk electrode. This work involves the experimental determination of the oxidation kinetics of sodium thiosulfate on a copper surface and the reduction kinetics of cupric/EDTA solutions as functions of various chemical environments 
and additive concentrations. No chemical analyses of the solutions or surface analyses of the electrodes were performed. All results are based strictly on the polarization data and knowledge of possible reactions and their theoretical open-circuit potentials. Following the presentation of the results will be a discussion of why we think electroless deposition did not occur. First off, a summary of electroless deposition and some reasons for trying thiosulfate are provided.

Electroless deposition (from the words electrodeless deposition ${ }^{1}$ and previously described by Paunovic $^{2}$ ) is a process for plating metals without the application of an outside agency (potential or current). In this process the reduction of a plating cationic species occurs simultaneously with the oxidation of a redox species. These reactions are to occur only through a catalytic surface and not homogeneously. An example is depicted by the following system which is currently used in industry.

$$
\begin{gathered}
\mathrm{Cu}^{2+}+2 \mathrm{e}^{-} \rightarrow \mathrm{Cu}, \\
\mathrm{HCHO}+2 \mathrm{OH}^{-} \rightarrow \mathrm{HCOOH}+\mathrm{H}_{2} \mathrm{O}+2 \mathrm{e}^{-} .
\end{gathered}
$$

A further understanding can be derived from the hypothetical polarization curves presented in figure 1.

Polarization curves are a measure of the kinetics of a particular system in terms of the current produced as a function of the potential applied to an electrode. Curves that rise sharply imply faster rates of reaction than those that rise more slowly. The solid 


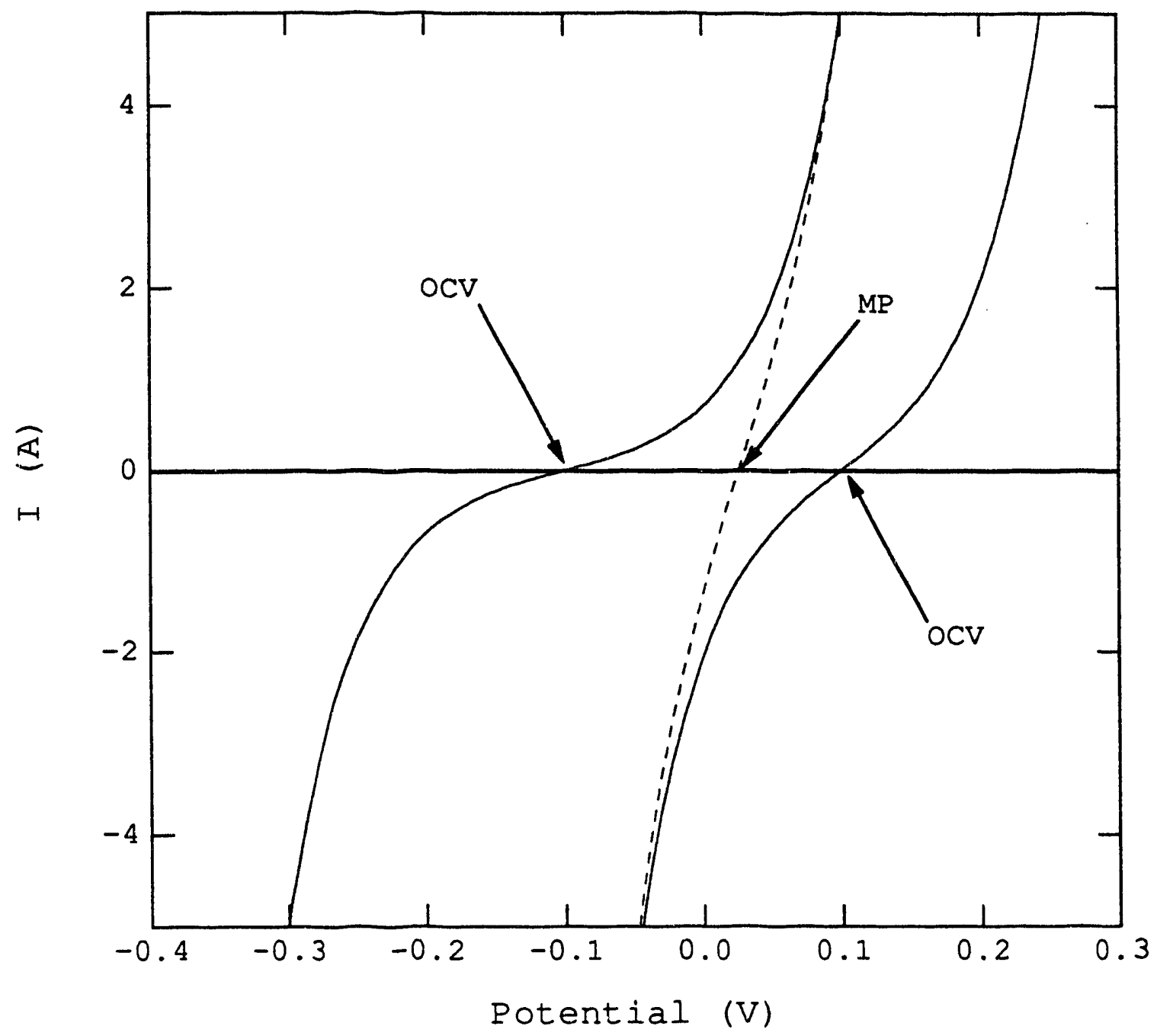

Figure 1. Sulid curves are hypothetical polarization curves. The dashed curve is the sum of the two curves. 
curve on the right of figure 1 exhibits faster kinetic properties than the solid curve on the left. Positive currents are considered anodic, while negative currents are considered cathodic. The points where the solid curves cross the abscissa are the open-circuit voltages of the respective reactions.

The solid curve on the right can be thought of as the polarization curve of copper in the absence of a reducing agent. A positive current suggests copper dissolution while a negative current suggests copper deposition. The solid curve on the left can be viewed as the polarization of formaldehyde in the absence of copper ions. The positive current suggests oxidation to formic acid while the negative current suggests the reverse reaction. The dashed line is the summation of the two curves. It is inherently steeper than both curves and approaches one or the other for potentials between the ien circuit potentials depending upon whether the current is positive or negative. This feature is due to the typically exponential dependence of the current on potential at currents higher than the exchange current but less than the limiting ourrent (see Newman ${ }^{3}$ chapter 8 for details on these concepts). The point at which the dashed curve crosses the abscissa is aptly named the mixed potential, and by its nature it must fall between the open-circuit potentials of the two reacting species. It is at this potential that electroless deposition would take place. That is the potential at which the oxidative current exactly balances the reductive current. Deviations from this potential may be due to the presence of another reactive 
species or to an interaction of the kinetics of the two primary reacting species.

The objectives of this research are to replace the formaldehyde with a more innocuous substance and then to characterize completely the kinetics of the system to determine the optimum plating conditions.

When choosing a reducing agent, it is a proviso that the species is oxidized at a lower potential than that at which the cation is reduced. The larger the difference between these two potentials, the larger the driving force for each reaction when the species are combined. But keep in mind, the driving force may be too large. Specifically, if the oxidation potential of the reducing agent is below the reduction potential of hydrogen ions, hydrogen gas is evolved. Not only does this result in poor reducing-agent utilization, it can lead to bubbles and nonuniform plating. Consequently, there is a window of oxidation potentials to which one is limited. The reducing agent and the oxidized products must also be soluble. These criteria eliminate a number of elements. Inspection of Pourbaix's table of electrochemical potentials ${ }^{4}$ reveals that only the sulfur, phosphorous, and carbon containing compounds meet these criteria. As has been stated, it is our intention to remove formaldehyde from the system; the focus of this research is on the sulfur compounds .

Because sulfate and sulfite are oxidized at relatively high potentials compared to copper reduction, they are eliminated as 
possibilities. Sulfide reacts readily with cuprous ions to form copper sulfide which precipitates from the solution. Thiosulfate, with its fairly low oxidation potential, is investigated.

Thiosulfate oxidizes to sulfite by the reaction ${ }^{5}$

$$
\mathrm{S}_{2} \mathrm{O}_{3}^{2-}+6 \mathrm{OH}^{-} \rightleftarrows 2 \mathrm{SO}_{3}^{2-}+3 \mathrm{H}_{2} \mathrm{O}+4 \mathrm{e}^{-}
$$

with an open-circuit potential of

$$
U^{\theta}=0.705-\frac{\mathrm{RT}}{\mathrm{F}} 2.303\left(\frac{6}{4} \mathrm{pH}+\frac{1}{4} \log \frac{\left[\mathrm{S}_{2} \mathrm{O}_{3}^{2}-\right]}{\left[\mathrm{SO}_{3}^{2-}\right]^{2}}\right)
$$

versus a SHE (standard hydrogen electrode). It is unstable at low $\mathrm{pH}$, decomposing to form sulfur and sulfite, ${ }^{6}$

$$
\mathrm{S}_{2} \mathrm{O}_{3}^{2-}(\mathrm{aq}) \rightleftarrows \mathrm{S}+\mathrm{SO}_{3}^{2-}(\mathrm{aq})
$$

This demands that the solution be maintained at a moderate to high $\mathrm{pH}$. Thiosulfate also forms a stable compound with $\mathrm{Cu}^{+}$. As we shall show, this reaction plays a pivotal role in the explanation of our experimental results.

To reduce cupric ions to copper at a catalytic surface with thiosulfate and yet prevent cupric ions from reacting homogeneously at high $\mathrm{pH}$ to form cupric oxide, a chelating agent must be adder to the solution. A chelating agent forms soluble ligands with copper ions and eliminates the reaction to an oxide. A number of chelating agents have been investigated in the literature; ${ }^{7,8,9,10}$ the most pervasive and the one used in this research is ethylenediamine tetraacetic acid (EDTA). This homogeneous reaction can be portrayed by the mechanism 


$$
\mathrm{Cu}^{2+}+[\mathrm{EDTA}]^{4-} \rightarrow \mathrm{Cu}[\mathrm{EDTA}]^{2-}
$$

and has an equilibrium constant of $6.31 \times 10^{18} \mathrm{~kg} / \mathrm{mol} .^{11}$ Such a large equilibrium constant suggests interpreting the copper species as existing exclusively as Cu[EDTA $]^{2-}$ in solution.

Polarization curves for the oxidation of thiosulfate and the reduction of $\mathrm{Cu}[\mathrm{EDTA}]^{2-}$ on a copper rotating disk electrode were obtained as described by the following experimental section.

\section{Experimental}

To obtain the polarization data for this system, a copper rotating disk, serial number 5144 of the Pine Instruments Company, with an active area of $0.382 \mathrm{~cm}^{2}$ is used as the working electrode for both the copper reduction and the thiosulfate oxidation. Before each experiment, the electrode is polished with emery paper, then $6 \mu \mathrm{m}$ diamond paste, $1 \mu \mathrm{m}$ diamond paste, and finally $1 / 4 \mu \mathrm{m}$ diamond paste. The disk is then rinsed with deionized water. The paste is Metaldi Diamond Compound by Buehler Ltd. catalog No.s 40-6162, 40-6122, and 40-6102, respectively, and is spread on Microcloth with adhesive backing by Buehler Ltd. "catalog No. 40-7218 attached to an Ecomet III by Buehler Ltd. polishing wheel. The electrode is screwed into a Pine Instruments Analytical Rotator model ASR2 before being immersed into the solution. All expe:iments are performed at $1000 \mathrm{rpm}$.

Solutions are made to $100 \mathrm{ml}$ and poured into the main body of an $\mathrm{H}-\mathrm{cell}$ and one its arms, which is to hold the counterelertrode. The counterelectrode is a coiled piece of $0.2 \mathrm{~cm}$ diameter copper wire 
with an exposed surface area greater than twenty times that of the working electrode. The reference-electrode compartment of the $\mathrm{H}$-celI is filled with $1 \mathrm{M} \mathrm{NaOH}$. $\mathrm{pH}$ measurements are made with a Beckman $\Phi$ $32 \mathrm{pH}$ Meter.

The reference electrode is a $\mathrm{Hg} / \mathrm{HgO} / 1 \mathrm{M} \mathrm{NaOH}$ electrode constructed with a piece of platinum wire encased in a glass tube with one end of the wire sticking out of the tube for electrical connection and the other end of the wire is fixed two centimeters from the other end of the tube. This end of the wire is encased in one centimeter of liquid mercury, which is then covered with $0.5 \mathrm{~cm}$ of red mercuric oxide. The remaining end of the tube is plugged with glass wool.

The H-cell is set in a stainless steel tub of water and thermostated to the desired temperature to within 0.1 degrees by a Thermomix 1441 heater by B. Braun Melsungen AG.

Most of the experiments are performed potentiostatically. The reference electrode is set at a potential relative to the grounded working electrode, and current is supplied through the counterelectrode to maintain that potential. The device used for setting the potential is a Potentiostat BC1200 by Stonehart Associates Inc. The potential of the reference electrode versus the working electrode is read from a digital display. The current in the system is also displayed as a potential and is interpreted as a current by dividing the potential by a set resistance. These data are also fed through a bus into a Macintosh II through a LabVIEW NB-MIO-16X-Multifunction 
16-Bit $A / D$ Board and displayed as graphs of potentials versus time with LabVIEW 2 software by National Instruments. After the potential is set to the desired potential and the system reaches steady state, the potential and current are recorded; the potential is then stepped to a new value. This technique was followed throughout the next section in deriving the polarization curves.

Results

Reduction of Copper Sulfate in the Presence of EDTA

Figure 2 presents five curves of the polarization data for copper deposition at $25^{\circ} \mathrm{C}$ for various concentrations of copper sulfate. The composition of the base-case solution is provided in table 1. The concentration of EDTA used in each experiment was equal to twice the concentration of the copper sulfate except for the experimental data listed as the first two curves in figure 2 where the copper concentration is zero and the EDTA is three times the copper concentration, respectively. The open-circuit potentials of the five

Table 1

\begin{tabular}{|ll|}
\hline species & conc. $\left(\mathrm{mol} / \mathrm{dm}^{3}\right)$ \\
\hline $\mathrm{CuSO}_{4}$ & 0.005 \\
$\mathrm{EDTA}$ & 0.015 \\
$\mathrm{NaOH}^{*}$ & 0.075 \\
$\mathrm{Na}_{2} \mathrm{SO}_{4}$ & 0.1 \\
\hline
\end{tabular}

* (adjusted to $\mathrm{pH}=11$ to 12 ) 
curves are recorded in table 2 in the same order as listed in the figure.

No trend was established between the open-circuit potential and. the quantity of copper sulfate in solution. The average value of the open-circuit potential is approximately $-0.16 \mathrm{~V}$. Inspection of the curves from the open-circuit potential to a potential of $-1.1 \mathrm{~V}$ reveals that the current remains very low $(<2 \mathrm{~mA})$ and is independent of the concentration of the copper sulfate species. This leads us to believe that there is a film on the electrode at these higher potentials - possibly an oxide. This is significant, for it indicates that in order to deposit copper electrolessly at $25^{\circ} \mathrm{C}$, the redox couple must have an open-circuit potential much less than the opencircuit potential of $-0.16 \mathrm{~V}$ (at least as low as $-1.1 \mathrm{~V}$. )

From $-1.1 \mathrm{~V}$ to $-1.3 \mathrm{~V}$, the current is roughly linearly dependent on the amount of copper in the solution: as the amount of copper doubles, so does the current. This behavior is expected for the deposi-

Table 2

\begin{tabular}{|lc|}
\hline curve & open-circuit potential (V) \\
\hline 1 & -0.150 \\
$2 *$ & \\
3 & -0.216 \\
5 & -0.115 \\
& -0.150 \\
\hline
\end{tabular}

* (not recorded) 


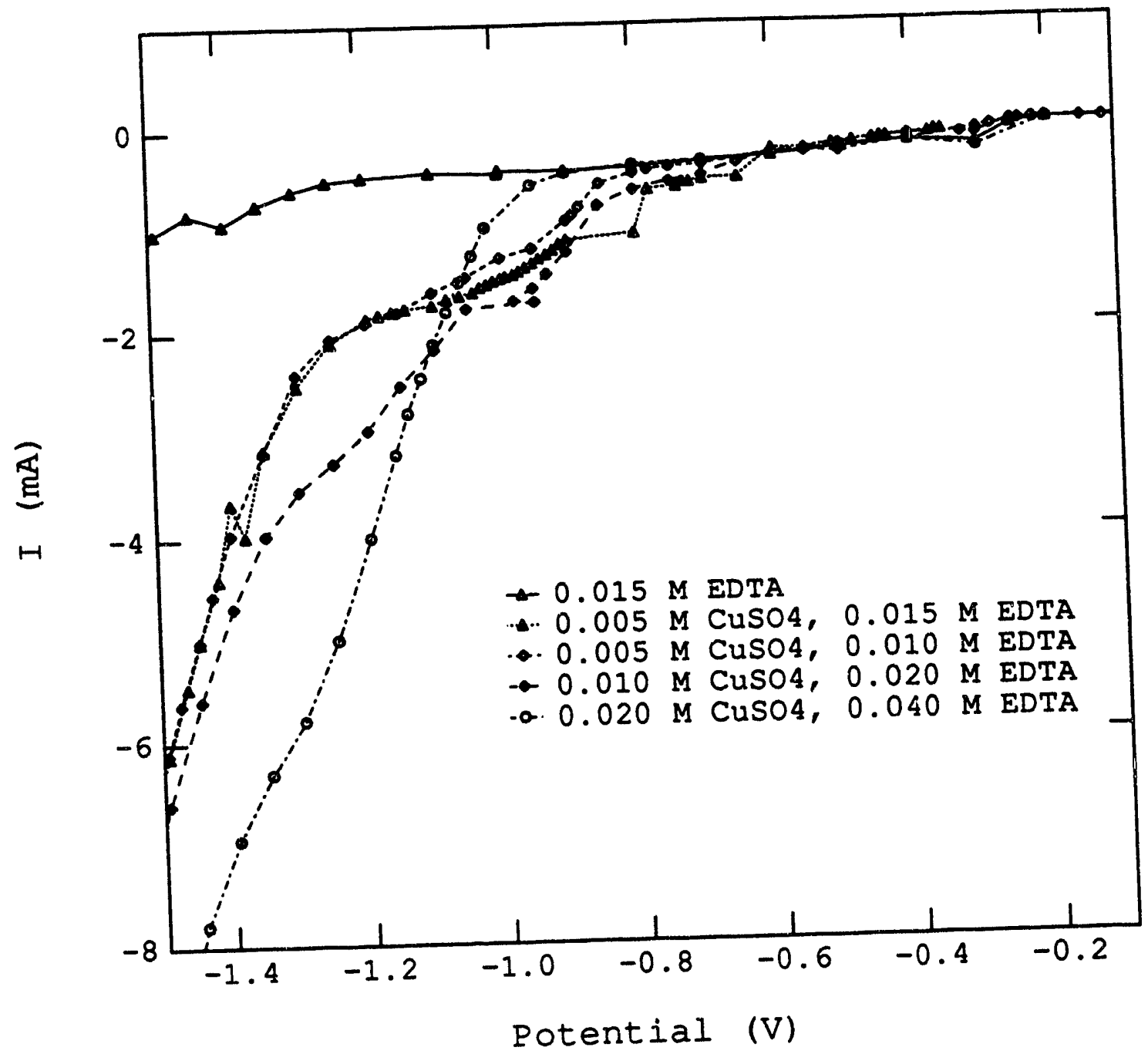

Figure 2. Cathodic current verses potential curves for solutions at a $\mathrm{pH}$ between 11 and 12 with $0.1 \mathrm{M} \mathrm{Na}_{2} \mathrm{SO}_{4}$ and the concentrations listed on the figure. 
tion of copper. At potentials lower than $-1.3 \mathrm{~V}$, the curves once again move toward each other losing their dependence on copper concentration and suggesting the formation of another type of film or changes in the surface morphology. The deposits at these low potentials are also powdery, a property that is normally undesirable. This information leads us to believe that the optimum potential for electroless copper plating at $25^{\circ} \mathrm{C}$ is between -1.1 and $-1.3 \mathrm{~V}$.

The second and third curves of figure 2 are representative of two experiments with the same amount of copper sulfate but different amounts of EDTA. The lines virtually fall on top of each other, which agrees with experiments by Molenaar et al. ${ }^{12}$ and Kondo et al., 9 who found that the rate of electroless deposition of copper is independent of the ratio of EDTA to copper in the solution as long as it is greater than one.

Oxidation of Sodium Thiosulfate

Polarization curves for the oxidation of thiosulfate for different concentrations of thiosulfate, supporting electrolyte $\left(\mathrm{Na}_{2} \mathrm{SO}_{4}\right)$, and $\mathrm{OH}^{-}$ions are presented in figure 3 . The base-case concentrations are provided in table 3 . The curves were derived by stepping the potential in $10 \mathrm{mV}$ intervals from the open-circuit potential to a potential beyond which the current drops significantly. This drop in current is indicative of the formation of an oxide film on the electrode surface which maintains poor catalytic properties for thiosulfate oxidation. The potential at which this drop off occurred was beiween -0.18 and $-0.16 \mathrm{~V}$. 


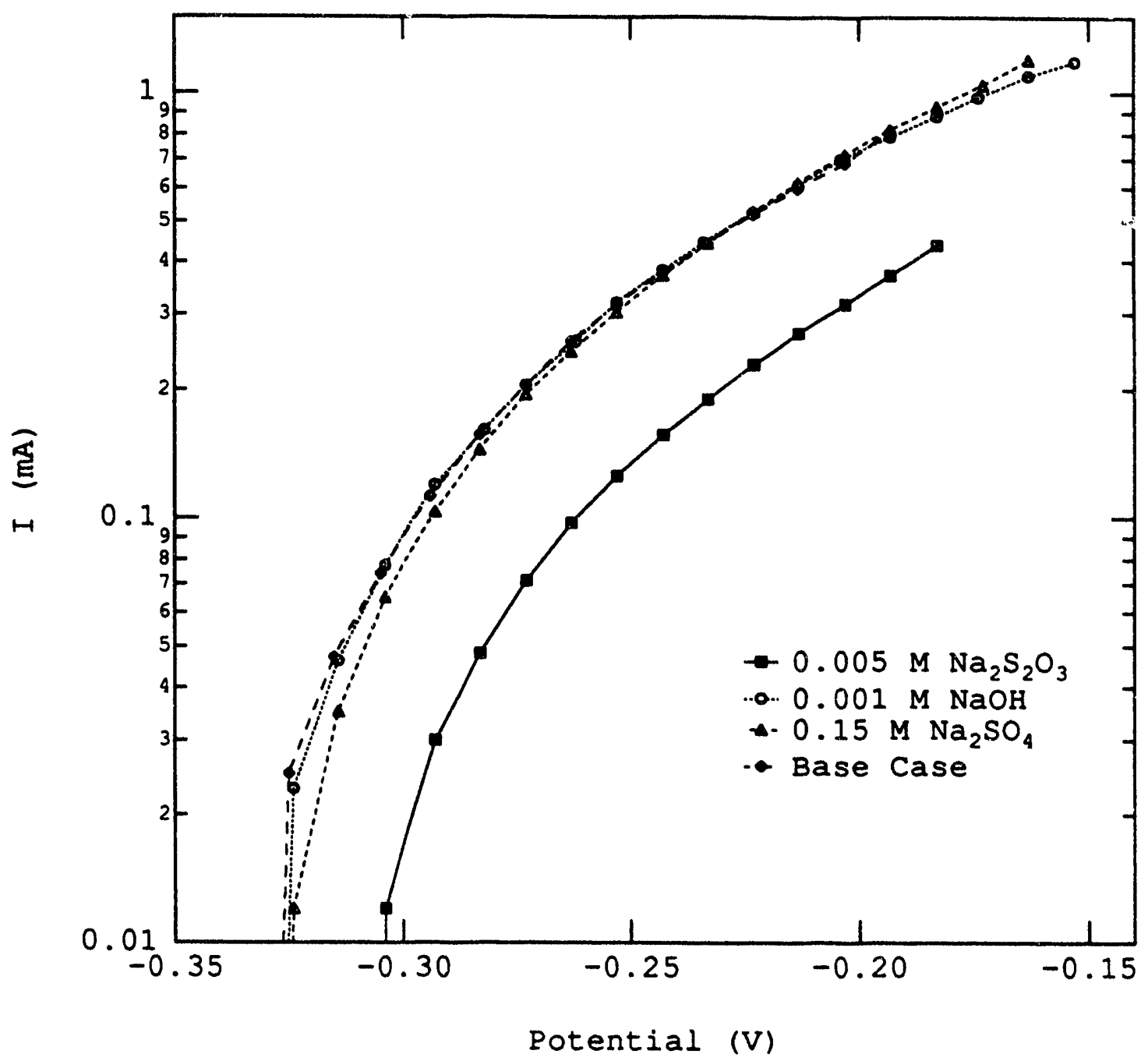

Figure 3. Anodic current-versus-potential curves of thiosulphate solutions containing species concentrations as given in table 3 with the differences listed on the figure. 
Table 3

\begin{tabular}{|ll|}
\hline species & conc. $\left(\mathrm{mol} / \mathrm{dm}^{3}\right)$ \\
\hline $\mathrm{Na}_{2} \mathrm{~S}_{2} \mathrm{O}_{3}$ & 0.010 \\
$\mathrm{NaOH}$ & 0.001 \\
$\mathrm{Na}_{2} \mathrm{SO}_{4}$ & 0.1 \\
\hline
\end{tabular}

The open-circuit potential, for solutions with $0.010 \mathrm{M} \mathrm{Na}_{2} \mathrm{~S}_{2} \mathrm{O}_{3}$, was measured at approximately $-0.335 \mathrm{~V}$ versus the $\mathrm{Hg} / \mathrm{HgO} / 1 \mathrm{M} \mathrm{NaOH}$ reference electrode, varying by $\pm 0.004 \mathrm{~V}$ in any given experiment. Without prior knowledge of the copper deposition kinetics, this low open-circuit potential suggests that electroless deposition of copper is plausible.

The data in figure 3 for various concentrations of $\mathrm{Na}_{2} \mathrm{SO}_{4}$ and $\mathrm{OH}^{-}$ions fall on top of each other. The current at a given potential for the solution with half as much thiosulfate is approximately half. Thus, the oxidation of thiosulfate is independent of the amount of supporting electrolyte and the $\mathrm{pH}$ and is directly proportional to the amount of thiosulfate in the solution.

The next step was the determination of the effect of EDTA on the oxidation of thiosulfate. Initial speculation suggested that the addition of EDTA should lower the current for a given potential by occupying surface sites for thiosulfate oxidation. In these experiments a solution of $0.005 \mathrm{M} \mathrm{Na}_{2} \mathrm{~S}_{2} \mathrm{O}_{3}, 0.1 \mathrm{M} \mathrm{Na}_{2} \mathrm{SO}_{4}, 0.005 \mathrm{M}$ EDTA, and 
enough $\mathrm{NaOH}$ to achieve a $\mathrm{pH}$ of 10 was prepared. The polarization data were obtained. In the following experiments the same concentrations of the species were maintained except for the EDTA, which was doubled (the $\mathrm{pH}$ was maintained at 10 by the addition of $\mathrm{NaOH}$ ). The doubling of the amount of EDTA continued until a concentration of $0.08 \mathrm{M}$ EDTA was realized. These curves are provided in figure 4. Contrary to our hypothesis, the current increased with the addition of EDTA. The rate of increase was proportional to the concentration of EDTA to the one-fifth power. Also, subsequent inspection of the disk revealed a slight amount of etching. The surface took on a more crystalline than polished appearance.

Now that we had the polarization curves for both the copper and the thiosulfate and their dependence on all the other species present in the solution, we combined the components into a single vessel.

Results of Combining Thiosulfate and Copper Ions in the Presence of Copper

In order to increase the likelihood of electrolessly plating copper, the concentrations of the active species were increased by at least an order of magnitude, see table 4. $100 \mathrm{ml}$ of this solution were poured into the H-cell and placed in the water bath, which was maintained at a temperature of $25^{\circ} \mathrm{C}$. The copper electrode was lowered into the solution, and the open-circuit potential versus the $\mathrm{Hg} / \mathrm{HgO} / 1 \mathrm{M} \mathrm{NaOH}$ reference electrode was monitored for the next three hours. The potential remained at approximately $-0.34 \mathrm{~V}$. Upon removal of the electrode, no electrolessly plated copper was found. On 


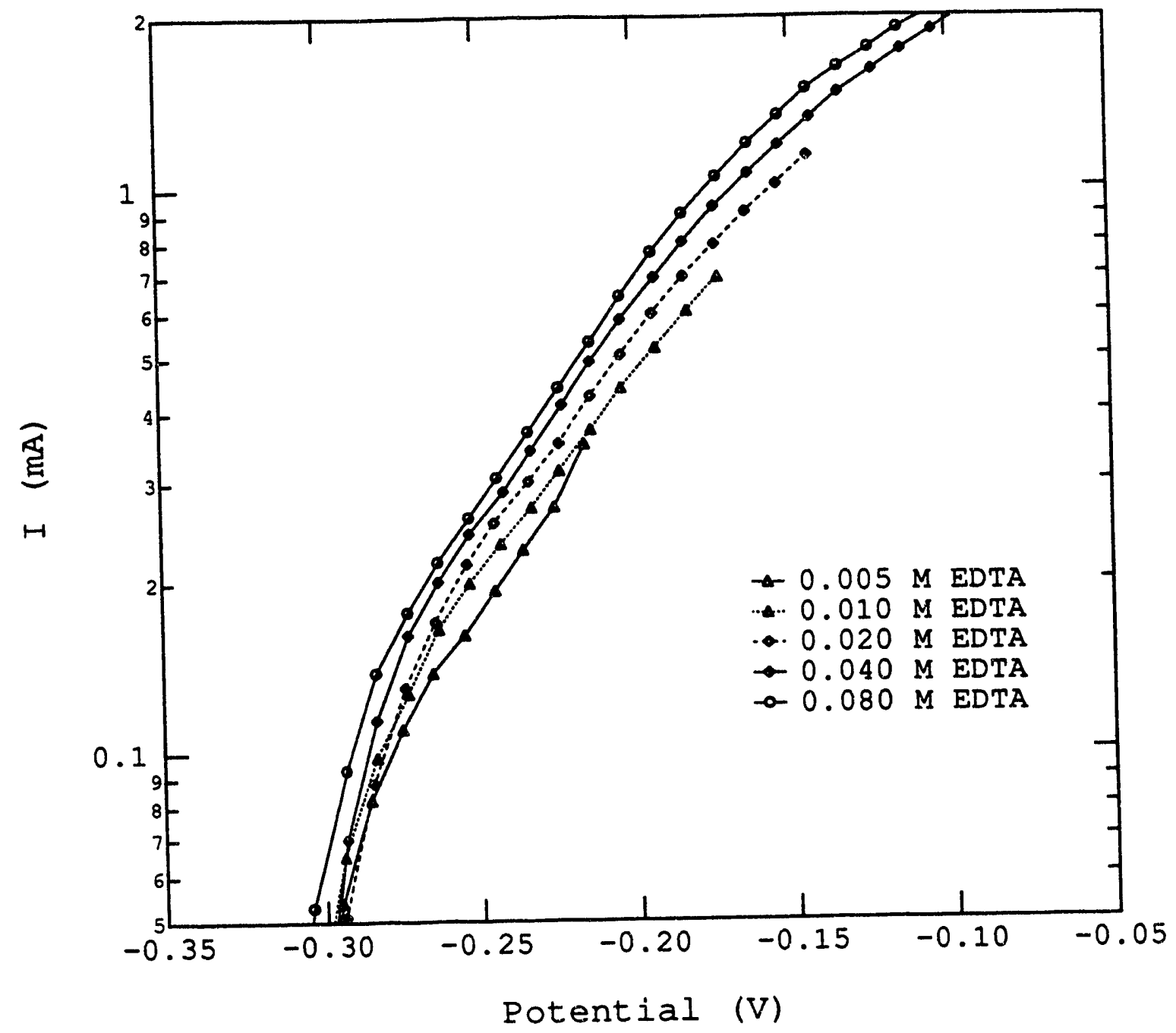

Figure 4. Anodic current vs. potential ofsolutions containing $0.005 \mathrm{M} \mathrm{Na}_{2} \mathrm{~S}_{2} \mathrm{O}_{3}, 0.01 \mathrm{M} \mathrm{Na}_{2} \mathrm{SO}_{4}$, at a $\mathrm{pH}$ of 10 with the concentrations of EDTA as listed on the figure. 
Table 4

\begin{tabular}{|lc|}
\hline species & conc. $\left(\mathrm{mol} / \mathrm{dm}^{3}\right)$ \\
\hline $\mathrm{CuSO}_{4}$ & 0.040 \\
$\mathrm{EDTA}$ & 0.080 \\
$\mathrm{Na}_{2} \mathrm{~S}_{2} \mathrm{O}_{3}$ & 0.5 \\
$\mathrm{NaOH}^{*}$ & 0.370 \\
$\mathrm{Na}_{2} \mathrm{SO}_{4}$ & 0.1 \\
$\mathrm{pH}^{2}$ & 11.92 \\
\hline
\end{tabular}

$*(\mathrm{pH}=11.92)$

the contrary, the electrode had partially dissolved. This led to the following investigation of possible reactions of $\mathrm{Cu}(\mathrm{s})$ with $\mathrm{S}_{2} \mathrm{O}_{3}^{2-}$. According to Kimura and Ishibashi, ${ }^{13}$ the reaction

$$
\mathrm{Cu}^{+}(\mathrm{aq})+\mathrm{S}_{2} \mathrm{O}_{3}^{2-}(\mathrm{aq}) \rightleftarrows\left[\mathrm{Cu}\left(\mathrm{S}_{2} \mathrm{O}_{3}\right)\right]^{-}(\mathrm{aq})
$$

takes place with an equilibrium constant of $2.24 \times 10^{10} \mathrm{~kg} / \mathrm{mol}$. In an aqueous phase, in the absence of complexing species, where the reactive species possibly present are $\mathrm{Cu}, \mathrm{Cu}^{+}$, and $\mathrm{Cu}^{2+}$, only $\mathrm{Cu}$ or $\mathrm{Cu}^{2+}$, depending on the concentration and potential, is present at equilibrium. $\mathrm{Cu}^{+}$is unstable. This is evident from the values of the standard potentials of the following reactions:

$$
\begin{gathered}
\mathrm{Cu} \rightleftarrows \mathrm{Cu}^{+}(\mathrm{aq})+\mathrm{e}^{-} \\
\mathrm{U}^{\theta}=0 . j 21 \mathrm{~V}+\frac{\mathrm{RT}}{\mathrm{F}} \ln \left[\mathrm{Cu}^{+}\right]
\end{gathered}
$$




$$
\begin{gathered}
\mathrm{Cu} \rightleftarrows \mathrm{Cu}^{2+}(\mathrm{aq})+\mathrm{e}^{-} ; \\
\mathrm{U}^{\theta}=0.337 \mathrm{~V}+\frac{\mathrm{RT}}{2 \mathrm{~F}} \ln \left[\mathrm{Cu}^{2+}\right] \\
\mathrm{Cu}^{+}(\mathrm{aq}) \rightleftarrows \mathrm{Cu}^{2+}(\mathrm{aq})+\mathrm{e}^{-} ; \\
\mathrm{U}^{\theta}=0.153 \mathrm{~V}+\frac{\mathrm{RT}}{\mathrm{F}} \ln \frac{\left[\mathrm{Cu}^{2+}\right]}{\left[\mathrm{Cu}^{+}\right]}
\end{gathered}
$$

(All potentials are vs. SHE.) Assuming concentrations of $1 \mathrm{M}, \mathrm{Cu}$ is the stable phase below $0.337 \mathrm{~V}$, and $\mathrm{Cu}^{2+}(\mathrm{aq})$ is the stable phase above $0.337 \mathrm{~V}$.

In the presence of thiosulfate, the following additional reactions with standard potentials must be considered.

$$
\begin{gathered}
\mathrm{Cu}+\mathrm{S}_{2} \mathrm{O}_{3}^{2-}(\mathrm{aq}) \rightleftarrows \mathrm{Cu}\left(\mathrm{S}_{2} \mathrm{O}_{3}\right)^{-}(\mathrm{aq})+\mathrm{e}^{-} \\
\mathrm{u}^{\theta}=-0.091 \mathrm{v}+\frac{\mathrm{RT}}{\mathrm{F}} \ln \frac{\left[\mathrm{Cu}\left(\mathrm{S}_{2} \mathrm{O}_{3}\right)^{-}\right]}{\left[\mathrm{S}_{2} \mathrm{O}_{3}^{2-}\right]} \\
\mathrm{Cu}\left(\mathrm{S}_{2} \mathrm{O}_{3}\right)^{-}(\mathrm{aq}) \rightleftarrows \mathrm{Cu}^{2+}(\mathrm{aq})+\mathrm{s}_{2} \mathrm{O}_{3}^{2-}(\mathrm{aq})+\mathrm{e}^{-} ; \\
\mathrm{U}^{\theta}=0.765 \mathrm{~V}+\frac{\mathrm{RT}}{\mathrm{F}} \ln \frac{\left[\mathrm{Cu}^{2+}\right]\left[\mathrm{S}_{2} \mathrm{O}_{3}^{-}\right]}{\left[\mathrm{Cu}\left(\mathrm{S}_{2} \mathrm{O}_{3}\right)^{-}\right]}
\end{gathered}
$$

(Both potentials are vs. SHE.) (Note: The potentials listed above are not applicable for solutions containing EDTA.) Again assuming concentrations of $1 \mathrm{M}, \mathrm{Cu}$ is the stable phase below $-0.091 \mathrm{~V}, \mathrm{Cu}^{2+}$ (aq) is the stable phase above $0.765 \mathrm{~V}$, and $\mathrm{Cu}\left(\mathrm{S}_{2} \mathrm{O}_{3}\right)^{-}$(aq) is the stable phase between these two voltages. Hence, the presence of thiosulfate stabilizes ine cuprous ion. The significance of this finding in rela- 
tion to the experiments is discussed.

\section{Discussion}

When a copper electrode is placed in a basic solution consisting solely of supporting electrolyte and an appropriate amount of sodium hydroxide to achieve a particular $\mathrm{pH}$ (no copper ions present), it is difficult to predict the open-circuit potential. However, if there is dissolved oxygen present, it is likely that a passive layer of $\mathrm{Cu}_{2} \mathrm{O}$ will form on the surface. When we perform this experiment, an open-circuit potential of $-0.056 \mathrm{~V}$ is measured, and little current is produced $(<5 \mu \mathrm{A})$ when the potential is swept $\pm 0.100 \mathrm{~V}$. This indicates that copper does not readily dissolve at potentials as high as $0.05 \mathrm{~V}$ and is further evidence that an oxide film exists.

Addition of sodium thiosulfate to the solution results in a drop of the open-circuit potential: a consequence of the thiosulfate to sulfite reaction. Furthermore, inspection of the electrode indicates that the copper surface does not retain its polished condition. This is perhaps due to the dissolution of the oxide layer and/or to the simultaneous dissolution of the $\mathrm{Cu}(\mathrm{s})$ to form $\mathrm{Cu}\left[\mathrm{S}_{2} \mathrm{O}_{3}\right]^{-}$. This may then explain the effects of EDTA on the polarization data obtained for solutions with thiosulfate. The curves of figure 4 are most likely a combination of thiosulfate oxidation and copper oxidation to cuprous thiosulfate. The EDTA may act as a catalyst in the latter reaction in removing the copper atoms from the surface or by promoting some stability of the copper ions. 
When thiosulfate is added to a solution of copper sulfate and EDTA at a pH of 10 to 12 and a copper electrode is then immersed into the solution, the copper dissolves. The open-circuit potential $(\approx-0.34 \mathrm{~V})$ is lower than the open-circuit potential of the copper in a copper sulfate/EDTA solution. This potential is not low enough for copper deposition (figure 2.) However, this potential is lower than that necessary to transform cupric ions to cuprous thiosulfate.

From this information it appears that when copper sulfate is added to a solution of sodium thiosulfate in the presence of copper, cupric ions are reduced to cuprous thiosulfate at a rate that is equal to the sum of the rates of the thiosulfate oxidation and of the copper oxidation to cuprous thiosulfate. This makes the use of thiosulfate as a possible reducing agent in electroless copper deposition unlikely.

\section{Conclusions}

The open-circuit potential of a copper electrode in a copper sulfate solution with EDTA at $25^{\circ} \mathrm{C}$ versus a $\mathrm{Hg} / \mathrm{HgO} / 1 \mathrm{M} \mathrm{NaOH}$ reference electrode is approximately $-0.16 \mathrm{~V}$. This value is independent of the amount of copper sulfate in the solution and, for the reasons discussed, indicates that there is probably an oxide on the electrode. The cathodic current does not become dependent on the concentration of copper sulfate until the potential is reduced to $-1.1 \mathrm{~V}$. The dependence of the current on concentration of copper sulfate is roughly linear to a potential of $-1.3 \mathrm{~V}$, where the dependence is 1ost. This potential window is considered the optimum potential 
range for plating copper at $25^{\circ} \mathrm{C}$ in an alkaline solution containing EDTA.

The open-circuit potential of a copper electrode in a solution containing $0.010 \mathrm{M} \mathrm{Na}_{2} \mathrm{~S}_{2} \mathrm{O}_{3}$ is $-0.337 \mathrm{~V}$. The application of an anodic potential produced polarization curves which were independent of the pH and dependent on the amount of EDTA to the one-fifth power and linearly dependent on the amount of thiosulfate present. These curves represent a combination of copper oxidation to cuprous thiosulfate and thiosulfate oxidation.

Finally, thiosulfate forms a stable soluble complex with $\mathrm{Cu}^{+}$. This complex allows for $\mathrm{Cu}^{2+}$ to be reduced to $\mathrm{Cu}^{+}$and copper to be oxidized to $\mathrm{Cu}^{+}$and thus negates the possibility of electroless copper deposition.

\section{References}

1. A. Brenner and G. Riddell, "Nickel Plating on Steel by Chemical Reduction," J. Research Nat. Bur. Standards, 37, July, 1946, and the A. E. S. Annual Proceedings p. 23 (1946).

2. M. Paunovic, "Electrochemical Aspects of Electroless Deposition of Metals," Plating, 55, 1161 (1968).

3. J. Newman, Electrochemical Systems, 2nd Edition, PrenticeHall, Englewood Cliffs, N. J. (1991). 
4. M. Pourbaix, Atlas of Electrochemical Equilibria in Aqueous Solutions, Second English Edition, National Association of Corrosion Engineers, Houston, Texas (1974).

5. J. V. Quagliano and L. M. Vallarino, Chemistry, 3rd Edition, Prentice-Hall, Inc., Englewood Cliffs, N.J. (1969).

6. B. H. Mahan, University Chemistry, 3rc' Edition, AddisonWesley Publishing Company, Menlo Park, Ca., p. 664 (1975).

7. A. Hur.g and K.-M. Chen, "Mechanism of Hypophosphite-Reduced Electroless Copper Plating," J. Electrochem. Soc., 136, 72 (1989).

8. R. Schumacher, J. J. Pesek, and O. R. Melroy, "Kinetic Analysis of Electroless Deposition of Copper," J. Phys. Chem., 89, 4338 (1985).

9. K. Kondo, J. Ishikawa, O. Takenaka, T. Matsubara, and M. Irie, "Acceleration of Electroless Copper Deposition in the Presence of Excess Triethanolamine," J. Electrochem. Soc., 138, 3629 (1991).

10. A. M. Mance, R. A. Waldo, and A. A. Dow, "Interactions of Electroless Catalysts with Plasma-Oxidized Surfaces of PolystyreneBased Resins," J. Electrochem. Soc., 136, 1667 (1989).

11. C. F. Bell, Principles and Applications of Metal Chelation, Clarendon Press, Oxford, p. 78 (1977).

12. A. Molenaar, M. F. E. Holdrinet, and L. K. H. van Beek, "Kinetics of Electroless Copper Plating with EDTA as the Complexing 
Agent for Cupric Ions," Plating, 61, 238 (1974).

13. M. Kimura and M. Ishibash1, "Kinetics of the Reaction of the Tris(oxalato)cobalt(III) Ion Induced by the Reaction between Copper(II) and Thiosulfate in Aqueous Solution," Inorganic Chimica Acta, 129, 69 (1987). 


\section{Chapter 5}

Electroless Deposition of Copper by Hypophosphite and Nickel Solutions

\section{Foreword}

Mixed-potential-versus-time curves are presented for various solutions consisting of copper and nickel ions. The data reveal that the addition of small amounts of copper ions to nickel solutions reduces the rate of electroless nickel deposition; whereas, there is a maximum concentration of nickel ions that can be added to a primarily copper sulfate solution to maximize electroless copper deposition.

\section{Introduction}

Formaldehyde is the most widely used and investigated reducing agent for the electroless deposition of copper. 1,2,3,4,5 (A review of plating including electroless copper plating is provided by Yung, et al. ${ }^{6}$ ) Formaldehyde is very volatile (normal boiling point is $-40^{\circ} \mathrm{C}$ ) and hazardous to one's health, but is used because it works. Hypophosphite is a widely used reducing agent for electroless nickel deposition, $7,8,9,10,11,12$ and is an ion in solution. It would be preferable to use hypophosphite for copper deposition as well; unfortunately, hypophosphite is not readily oxidized on copper surfaces. 13 This research is an extension of previous attempts by other researchers $^{14,15}$ to use hypophosphite as a possible reducing agent for elec- 
troless copper deposition while in the presence of nickel ions. Dubinl 16 has shown that hypophosphite can be used as a reducing agent for nickel in the presence of a small amount of copper; this was also reinvestigated.

The combination of reactants $-\mathrm{Ni}^{2+}, \mathrm{Cu}^{2+}$, and $\mathrm{H}_{2} \mathrm{PO}_{2}^{-}-$in the presence of a palladium chloride activated surface initiates the deposition of nickel, which then promotes the simultaneous deposition of copper. This work presents the mixed potentials versus time for solutions of various concentrations of copper sulfate, hypophosphite, and nickel solutions. It was found that copper poisons the nickel deposition and that there is an optimum amount of nickel sulfate that can be added to a copper solution to maximize the duration of copper deposition.

\section{Experimental}

The equipment used in this research is identical to that used in the previous study of thiosulfate oxidation on copper (Chapter 4), and, hence, shall not be repeated here. The one major equipment difference is the electrode, which is a palladium ring-disk electrode of the Pine Instruments Company, serial number 297, having a total surface area of $0.382 \mathrm{~cm}^{2}$. The electrode is prepared by polishing, as described in the previous chapter, and activating. Activation is achieved by dipping the electrode into an activating solution, as described in table 1, for two minutes, prior to each experiment. 
Table 1

\begin{tabular}{|ll|}
\hline \multicolumn{1}{|c|}{ species } & amount \\
\hline $\mathrm{PdCl}_{2}$ & $0.1 \mathrm{~g}$ \\
Glacial acetic acid & $50 \mathrm{ml}$ \\
$\mathrm{HCl}(37 \%)$ & $0.5 \mathrm{ml}$ \\
Deionized $\mathrm{H}_{2} \mathrm{O}$ & to $100 \mathrm{ml}$ \\
\hline
\end{tabular}

The experimental technique differs substantially from the work in the previous chapter. Here, the circuit is left open, and the potential of the palladium disk versus a $\mathrm{Hg} / \mathrm{HgO} / 1 \mathrm{M} \mathrm{NaOH}$ reference electrode is monitored as a function of time. These data are relayed from the potentiostat directly to a Macintosh II through coaxial cables and a LabVIEW NB-MIO-16X multifunction 16-bit A-D board. The data are stored on a disk and displayed on the terminal using LabVIEW 2 software by National Instruments.

\section{Results and Discussion}

Nickel Chloride Solutions with Sparing Amounts of Copper Sulfate

As mentioned, hypophosphite is used as a reducing agent for electroless nickel deposition (due primarily to its favorable kinetic properties on nickel surfaces), but is a poor agent for electroless copper (for the inverse reason). The purpose of these experiments is to determine the extent to which copper sulfate can be added to nickel/hypophosphite solutions to maximize copper deposition yet 
still achieve a moderate rate of codeposition. Thus, solutions with relatively large amounts of nickel chloride and hypophosphite are prepared with various amounts of copper sulfate. The concentrations of the species used in each experiment"are taken from the work of Dubin $^{16}$ and are either listed on figure 1 or are provided below.

The experiment with no copper sulfate also contains $0.37 \mathrm{M}$ citric acid. The experiments with copper sulfate also contain $0.33 \mathrm{M}$ glycine and $0.037 \mathrm{M}$ sodium acetate. Each of the solutions is adjusted to the $\mathrm{pH}$ listed on the figure with the appropriate amount of sodium hydroxide.

In the experiment in which no copper sulfate is present, the open-circuit potential starts at $-0.65 \mathrm{~V}$ and quickly decreases (within the next 200 seconds) to $-0.97 \mathrm{~V}$. Inspection of the electrode after an hour of being immersed reveals a nickel covered surface with bubbles vigorously evolving from portions of the electrode still covered with solution.

The mixed potential diagrams of solutions which contain increasing amounts of copper sulfate are also provided in figure 1 . One sees that as the concentration of copper ions increases, so does the potential. Inspection of the electrodes after each experiment reveals less deposit as the copper content of the solution is increased. These data lead to the following discussion. 


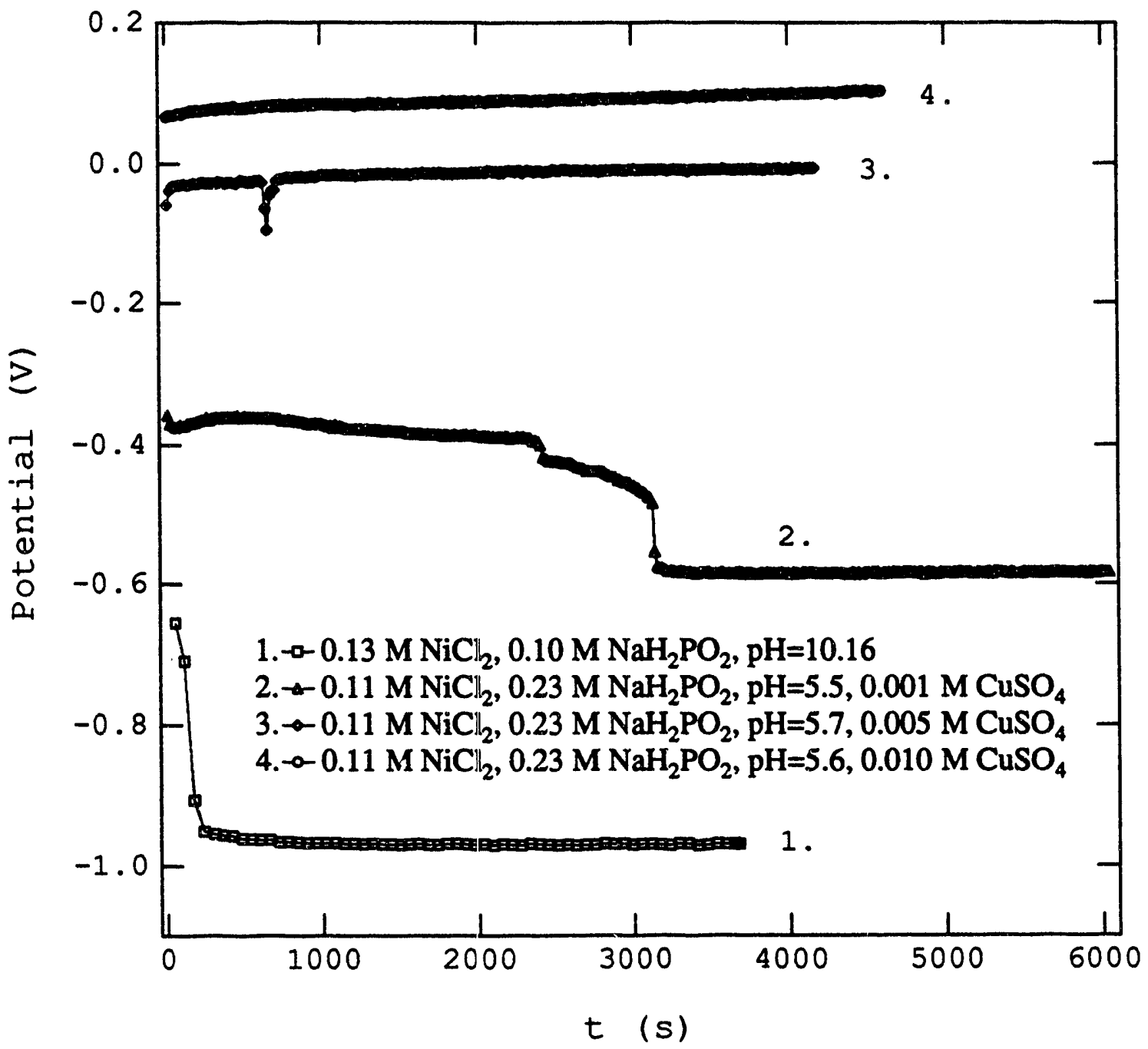

Figure 1. Mixed potential curves of nickel predominant solutions. Concentrations of additives are listed in the text. 
Discussion

According to data found in vetter, ${ }^{17}$ palladium is a slightly better catalyst for hydrogen evolution than is nickel, which is better still than copper. The hydrogen to hydrogen ion and hypophosphite to phosphite open-circuit potentials are functions of solution composition and are independent of the surface present. However, the surface does play a large role in the kinetic properties. The nickel deposition open-circuit potential is a strong function of the surface since this reaction consists of a dissolved species reduced to a solid species. This information leads to the following arguments. For the solution without copper ions, nickel is initially deposited on the electrode, which brings about the immediate decrease in potential (curve 1, figure 1.) This new surface must then be either more kinetically favorable for hypophosphite reduction, or less kinetically favorable for nickel deposition or hydrogen evolution, or a combination of the three. Since hydrogen evolution on the nickel surface is prevalent, it is unlikely that reduction of the hydrogen reaction overpotential is responsible for the large change of potential (over $300 \mathrm{mV}$. )

However, the drop in potential combined with the vigorous bubble evolution and the obvious presence of nickel does suggest a shift to a much more kinetically favorable state of hypophosphite oxidation. Therefore, we conclude that this change in potential is due mainly to an increase of the hypophosphite kinetics on nickel relative to palladium. 
In discussing the data it is important to consider that the palladium electrode was "activated;" that is it was dipped in the activating solution before each experiment. And that the electrode was relatively inactive without this step. It thus appears that the activator is creating a finite number of activation sites. These sites are probably utilized by all four components, hypophosphite, protons, and copper and nickel ions. For the solution with no copper present, it appears that the nickel and hypophosphite compete for these sites, but as the nickel is deposited, it creates more active sites for hypophosphite oxidation. The potential drops.

For solutions with copper ions, it appears that the copper deposition also competes for these activation sites. However, the copper does not catalyze the hypophosphite; and, therefore, as the experiment proceeds, the copper ions consume the activation sites and little deposition is seen. In the case of the solution with the smallest copper composition (curve 2, figure 1), the nickel can compete with the copper in consuming sites; and once all the sites are occupied (after $3000 \mathrm{~s}$, see figure 1), the nickel can still act as a catalytic site for both the nickel deposition and the hypophosphite oxidation. Thus the potential abruptly drops nearly $100 \mathrm{mV}$ after 3000 seconds, and nickel deposition and hydrogen evolution proceed simultaneously with an increased rate of hypophosphite oxidation. (This provides further support that nickel is an excellent catalyst for hypophosphite oxidation and that copper is a poor one). 
Copper Sulfate Solutions with Sparing Amounts of Nickel Sulfate

Solutions which primarily consist of copper sulfate, hypophosphite, and boric acid were prepared with various sparing amounts of nickel chloride. The purpose of these experiments is to deposit copper with hypophosphite and to elucidate the effect that nickel plays in the process. The base-case concentration of components, as duplicated from Hung et al. ${ }^{14}$ is provided in table 2. The opencircuit-versus-time data for various solutions containing primarily copper ions and hypophosphite as a reducing agent are provided in figure 2 .

For the base-case solution composition, the open-circuit potential starts at a low potential, $-0.7 \mathrm{~V}$, which is conducive to high rates of deposition. However, the potential steadily rises, and after the first 900 seconds the potential has essentially reached a steady value of $-0.1 \mathrm{~V}$. Inspection of the electrode aftex approxi-

Table 2

\begin{tabular}{|ll|}
\hline species & conc. $\left(\mathrm{mol} / \mathrm{dm}^{3}\right)$ \\
\hline $\mathrm{CuSO}_{4}$ & 0.024 \\
$\mathrm{NiSO}_{4}$ & 0.002 \\
$\mathrm{H}_{3} \mathrm{BO}_{3}$ & 0.5 \\
$\mathrm{Citric} \mathrm{Acid}_{2}$ & 0.056 \\
$\mathrm{NaH}_{2} \mathrm{PO}_{2}$ & 0.27 \\
\hline
\end{tabular}

(solutions adjusted to a $\mathrm{pH}$ of $9.2 \mathrm{w} / \mathrm{NaOH}$ ) 


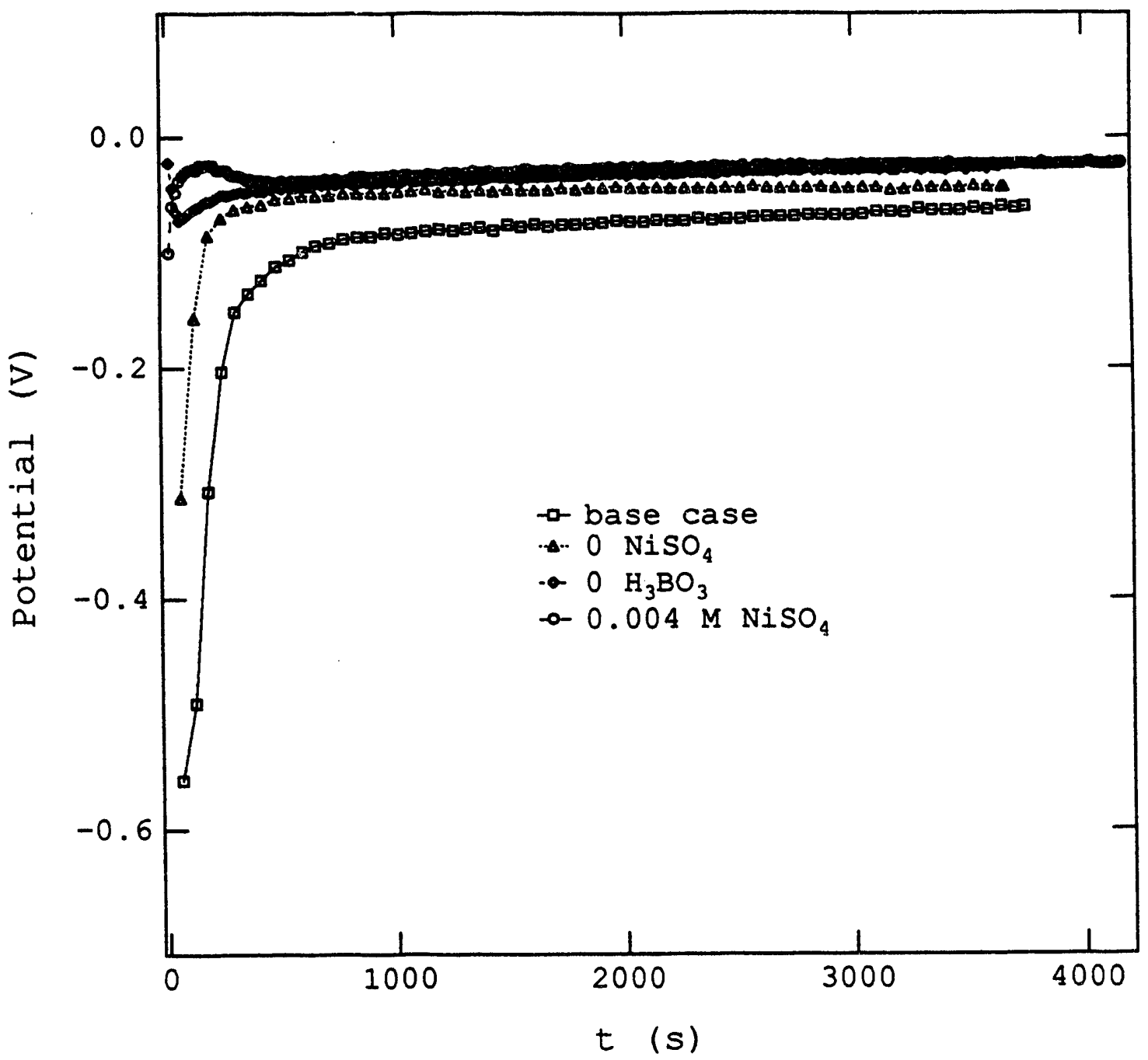

Figure 2. Mixed potential versus time for prodomiantly copper solutions. Base Case concentrations: $0.024 \mathrm{M} \mathrm{CuSO}_{4} ; 0.002 \mathrm{M}$ $\mathrm{NiSO}_{4} ; 0.5 \mathrm{M} \mathrm{H}_{3} \mathrm{BO}_{3} ; 0.056 \mathrm{M}$ Citric Acid; $0.27 \mathrm{M} \mathrm{NaH}_{2} \mathrm{PO}_{2} ; \mathrm{NaOH}$ to a $\mathrm{pH}=9.2$. Adjustments from these concentrations are listed on the figure. 
mately one hour reveals a faint copper surface.

For the solution with no nickel present, the potential again starts at a low potential, $-0.7 \mathrm{~V}$, and rises quickly, within 200 seconds, to a steady potential of $-0.07 \mathrm{~V}$.

The solution with twice as much nickel starts at a potential of $-.8 \mathrm{~V}$ (observed but not stored or displayed in figure 2), but rose very rapidly (within 10 seconds) to a potential of $-0.06 \mathrm{~V}$.

Lastly, the solution with no boric acid does very little, hovering around $-0.06 \mathrm{~V}$.

\section{Discussion}

Again it appears that there is competition for the activation sites. For the base case, the copper competes for the sites with the nickel, and codeposition conti.ues for approximately 900 seconds. In the experiment where the nickel concentration has been doubled, nickel ions consurne most of the sites but there is not much nickel present in solution to create an appreciable amount of current. For the case where no nickel is present, the copper consumes all the sites, and the current stops because the kinetics for hypophosphite oxidation on copper are poor. The last curve mentioned is the mixed potential of a solution of copper and nickel but with no boric acid present. According to Ohno et al., 13 boric acid is needed for the nickel deposition and the hypophosphite oxidation. These data agree with his argument to the extent that nothing occurs without the presence of boric acid. 


\section{Further Discussions}

The transient experiments presented here showed little reproducibility. This is most likely due to the problems faced in reproducing the surface prior to each experiment. The surface was polished and dipped in $100 \mathrm{ml}$ of activating solution, which was therefore depleted of some Pd ions each time it was used. The solution was never replenished. The number of activation sites was poorly controlled by this technique. The electrode was then exposed to air as it was transferred to the rotating-disk apparatus. Oxvgen in the atmosphere could have a significant effect on the activated palladium surface by chemical reaction or adsorption. This too would obviously affect the ability of reproducing the same surface of the electrode for e.ach run.

Experirental results provided in this manuscript are those for which the lowest potential was observed icaediately after the electrode was immersed in the solution. For some experiments, the potential started at a relatively high potential $(-0.1 \mathrm{~V})$ and remained there. In those cases, the electrode was removed from the electroless solution and redipped in the activating solution. As a consequence of this experimental artifact of poor reproducibility, we are not overly confident of the arguments that have been presented.

\section{Conclusions}

\footnotetext{
Open-circuit potentials versus time of various solutions containing copper and nickel are presented. It was demonstrated for
} 
solutions consisting primarily of nickel ions, that as the copper concentration is increased, the mixed potential increases and is accompanied by a decrease in nickel deposition. This was attributed to the simultaneous deposition of copper, which is a poor catalytic surface for hypophosphite oxidation.

For solutions consisting primarily of copper sulfate, a maximum in copper deposition is observed as nickel sulfate is added. This effect was attributed to nickel consumption of most the activation sites which prohibits further deposition of copper. Surface preparation plays an important role in the system performance.

\section{References}

1. R. Schumacher, J. J. Pesek, and O. R. Melroy, "Kinetic Analysis of Electroless Deposition of Copper," J. Phys. Chem., 89, $4338-42$ (1985).

2. M. Paunovic, "Potentiostatic High Overpotential Studies of Copper Deposition from Electroless Copper Solutions," J. Electrochem. Soc., 132, 1155-57 (1985).

3. B. J. Feldman and 0 . R Melroy, "The Mechanism of Electroless Cu Deposition: Extraction of the Oxidative and Reductive Electrochemical Half-Cell Currents from a Complete Bath," J. Electrochem. Soc., $136,640-43(1989)$.

4. M. Matsuoka, K. Iwamoto, and T. Hayashi, "Deposition Rate of Electroless Copper Monitored by a Computer-aided Coulostatic Method," 
Plating and Surface Finishing, 78, 64-68 (1991).

5. T. Ogura, "Mechanism of Copper Deposition in Electroless Plating," Langmuir, 6, 1709-10 (1990).

6. E. K. Yung, L. T Romankiw, and R. C. Alkire, "Plating of Copper into Through-Holes and Vias," J. Electrochem. Soc., 136, 206 15 (1989).

7. F. M. Donahue, "Interactions in Mixed Potential Systems," J. Electrochem. Soc., 119, $72-73$ (1972).

8. P.-L. Pai and C. H. Ting, "Selectively Deposited Nickel Film for Via Filling," IEEE Electron Dev. Lett., 10, 257-59 (1989).

9. C. H. Ting and M. Paunovic, "Selective Electroless Metal Deposition for Integrated Circuit Fabrication," J. Electrochem. Soc., $136,456-62(1989)$.

10. T. Osaka, I. Koiwa, M. Usuda, K. Arai, and I. Saito, "Behavior of the Electroless Composite Films Plated from a Ni-P Bath with Metallic Dispersion of $\mathrm{Zr}$ and $\mathrm{Nb}$ Powders, " J. Electrochem. Soc., $136,1124-28$ (1989).

11. K. - L Lin and P.-J. Lai, "The Crystalization of an Electroless Ni-P Deposit," J. Electrochem. Soc., 136, 3803-09 (1989).

12. V. Dubin, "Electroless Ni-P Deposition on Silicon with Pd Activation," J. Electrochem. Soc., 139, $1289-94$ (1992). 
13. I. Ohno, O. Wakabayashi, and S. Haruyama, "Anodic Oxidation of Reductants in Electroless Plating," J. Electrochem. Soc., 132 , $2323-2330$ (1985).

14. A. Hung and I. Ohno, "Electrochemical Study of Hypophosphite-Reduced Electroless Copper Deposition," J. Electrochem. Soc., 137, 918-921 (1990).

15. A. Hung and K.-M. Chen, "Mechanism of Hypophosphite-Reduced Electroless Copper Plating," J. Electrochem. Soc., 136, 72-75 (1989).

16. V. Dubin, "Selective Electroless $\mathrm{Ni}-\mathrm{Cu}(\mathrm{P})$ Deposition for Via Hole Filling and Conductor Pattern Clodding in a VLSI Multilevel Interconnection Structures," J. Electrochem. Soc., 139, 633-638 (1992).

17. K. J. Vetter, Electrochemical Kinetics: Theoretical and Experimental Aspects, Academic Press Inc., New York, pp. 576-77 (1967). 

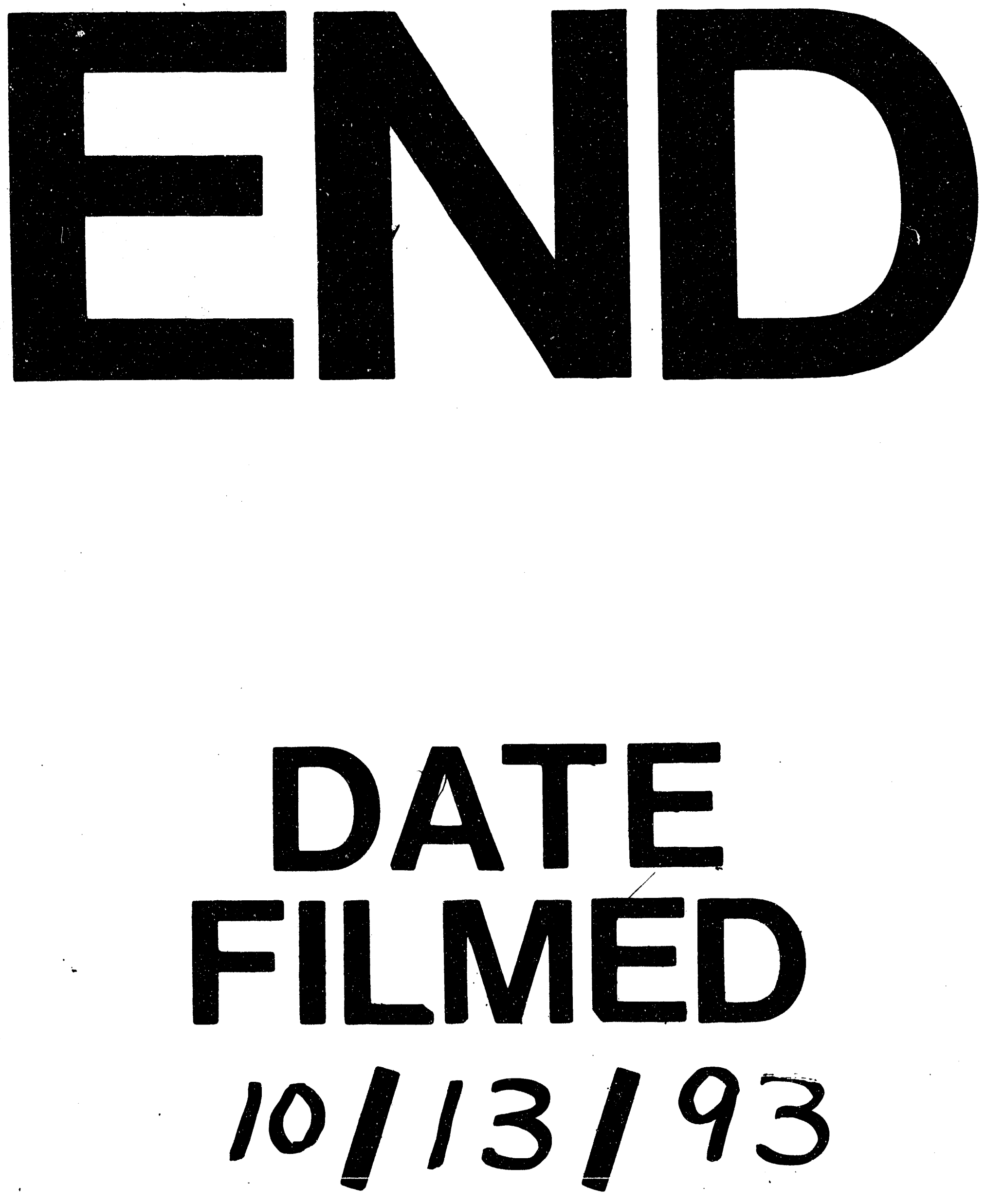

1 
